\title{
Optical gas sensing: a review
}

\author{
Jane Hodgkinson* and Ralph P Tatam
}

Department of Engineering Photonics, School of Engineering, Cranfield University, Cranfield, Bedfordshire, MK43 OAL, UK.

¡.hodgkinson@cranfield.ac.uk

\begin{abstract}
The detection and measurement of gas concentrations using the characteristic optical absorption of the gas species is important for both understanding and monitoring a variety of phenomena from industrial processes to environmental change. This article reviews the field, covering several individual gas detection techniques including non-dispersive infrared (NDIR), spectrophotometry, tunable diode laser spectroscopy and photoacoustic spectroscopy. We present the basis for each technique, recent developments in methods and performance limitations. The technology available to support this field, in terms of key components such as light sources and gas cells, has advanced rapidly in recent years and we discuss these new developments. Finally, we present a performance comparison of different techniques, taking data reported over the preceding decade, and draw conclusions from this benchmarking.
\end{abstract}

\section{Introduction}

Gas detection has an impact across a wide range of applications. Early markets have included the process and petrochemical industries, where sensors are used to ensure safety (eg via detection of toxic or flammable gases), monitor feedstocks and measure key species in products and processes, some of which can be rapidly changing ${ }^{[1]}$. Use of high sensitivity gas detectors is widespread in atmospheric science, where they are used to measure and understand the profile and pathways of different gas species including greenhouse gases ${ }^{[2]}$. Various potential biomarker gases are also under study for use in breath diagnostics, including nitric oxide (NO), ethane, ammonia $\left(\mathrm{NH}_{3}\right)$, and many more $^{[3]}$.

Quantitative detection of gases is traditionally dominated by laboratory analytical equipment such as gas chromatographs, with sampling that precludes real-time data ${ }^{[3]}$, or small ultra-low-cost devices such as pellistors, semiconductor gas sensors or electrochemical devices. Pellistors are robust devices that respond to combustion on a catalyst bead ${ }^{[4]}$; they perform well in detecting flammable gases close to the lower explosive limit, however suffer from zero drift at parts per million (ppm) levels. Semiconductor gas sensors can be highly sensitive at the low ppm level ${ }^{[5]}$, however these also suffer from drift and cross-respond to other gases and changing humidity levels. Electrochemical gas 
sensors can be relatively specific to individual gases and sensitive at ppm or ppb levels ${ }^{[6]}$, however they have limited lifetimes and also suffer from some known cross-response issues, eg to humidity.

In contrast, gas sensors based on optical absorption offer fast responses (time constants below $1 \mathrm{~s}$ are possible), minimal drift and high gas specificity, with zero cross-response to other gases as long as their design is carefully considered. Measurements can be made in real time and in situ without disturbing the gas sample, which can be important in process control ${ }^{[7]}$. Because the transduction method makes a direct measurement of a molecule's physical properties (its absorption at a specific wavelength), drift is reduced and, because the incident light intensity can be determined, measurements are self-referenced, making them inherently reliable. In this way, optical gas sensing fills an important gap between lower cost sensors with inferior performance and high end laboratory equipment.

Table 1. Examples of applications for methane detection, illustrating the need for gas measurement over different concentration ranges. Not all applications currently employ optical techniques.

\begin{tabular}{|c|c|c|c|}
\hline Application & $\underline{\text { Significant issues }}$ & $\begin{array}{l}\text { Required } \\
\text { concentration range }\end{array}$ & $\begin{array}{l}\text { Example } \\
\underline{\text { ref }}\end{array}$ \\
\hline $\begin{array}{l}\text { Process control: gas quality, ie } \\
\text { measurement of natural gas } \\
\text { composition for regulation, } \\
\text { metering and custody transfer }\end{array}$ & $\begin{array}{l}\text { Accuracy to "fiscal standards" } \\
(0.1 \%)\end{array}$ & $70-100 \%$ vol & [8] \\
\hline $\begin{array}{l}\text { Safety: purging gas pipes to } \\
\text { avoid explosions and ensure } \\
\text { pilot lights remain burning. }\end{array}$ & $\begin{array}{l}\text { Accuracy eg to } \pm 5 \% \text { vol at } \\
50 \% \text { vol }\end{array}$ & $1-100 \%$ vol & [9] \\
\hline $\begin{array}{l}\text { Process control: monitoring } \\
\text { combustion processes }\end{array}$ & $\begin{array}{l}\text { Accuracy in a wide range of } \\
\text { temperature and pressures }\end{array}$ & $0.1-100 \% \mathrm{vol}$ & [10] \\
\hline $\begin{array}{l}\text { Safety: quantification of gas } \\
\text { leaks with respect to the lower } \\
\text { explosive limit (LEL) of } 4.9 \% \text { vol }\end{array}$ & $\begin{array}{l}\text { Accurate at action points eg } \\
20 \% \text { LEL ( } 1 \% \text { vol) for } \\
\text { evacuation of buildings }\end{array}$ & $0.1-5 \% \mathrm{vol}$ & [11] [12] \\
\hline $\begin{array}{l}\text { Safety: location of gas leaks, } \\
\text { often outdoors }\end{array}$ & $\begin{array}{l}\text { Reliable zero } \\
\text { Limit of detection approaching } \\
1 \mathrm{ppm}\end{array}$ & $1-10,000$ ppm & [12] [13] \\
\hline $\begin{array}{l}\text { Process / environment: } \\
\text { quantification of residual } \\
\text { methane in flares, for carbon } \\
\text { trading }\end{array}$ & $\begin{array}{l}\text { Repeatability } 100 \mathrm{ppb} \\
\text { Background methane } 1.8 \mathrm{ppm} \\
\text { (higher when close to sources) }\end{array}$ & $\begin{array}{l}100 \mathrm{ppb}-1,000 \mathrm{ppm} \\
\text { (plus background } \\
\text { level of } 1.8 \mathrm{ppm} \text { ) }\end{array}$ & [14] \\
\hline $\begin{array}{l}\text { Environmental modelling: } \\
\text { measurement of the methane } \\
\text { background of } 1.8 \mathrm{ppm}\end{array}$ & $\begin{array}{l}\text { Comparison with historic data. } \\
\text { Accuracy of } 0.1-5 \% \text { of reading } \\
\text { required. }\end{array}$ & $\begin{array}{l}30 \mathrm{ppb}-3 \mathrm{ppm} \\
\text { (plus background } \\
\text { level of } 1.8 \mathrm{ppm} \text { ) }\end{array}$ & [15] \\
\hline $\begin{array}{l}\text { Environmental modelling: } \\
\text { methane flux measurement by } \\
\text { eddy covariance technique }\end{array}$ & $\begin{array}{l}\text { Correlation with local } \\
\text { atmospheric eddy currents at } \\
\text { data rates }>10 \mathrm{~Hz}\end{array}$ & $\begin{array}{l}5 \mathrm{ppb}-25 \text { ppm } \\
\text { (plus background } \\
\text { level of } 1.8 \mathrm{ppm} \text { ) }\end{array}$ & [16] \\
\hline
\end{tabular}


Gas detection applications can cover a very wide range of gas concentrations. The concentration is typically expressed as a proportion in air (or some other matrix) by volume. Since most gases at standard temperature and pressure behave as ideal gases to a high degree, this is also equal (or almost equal) to the molar concentration in the matrix. To put gas concentrations into context, we can take the example of one gas species (methane) with a variety of applications, each demanding measurement over a different concentration range. Several examples are summarised in Table 1. Concentrations are expressed as \%vol (\% by volume), ppm (parts per million by volume; 1 part in $10^{6}$ ), ppb (parts per billion by volume; 1 part in $10^{9}$ ) or ppt (parts per trillion by volume, 1 part in $10^{12}$ ).

In this review, we discuss commonly used techniques in gas sensing based on measurement of optical absorption at specific wavelengths. These are non-dispersive gas sensing including nondispersive infra-red (NDIR), spectrophotometry, tunable diode laser spectroscopy (TDLS) and photoacoustic spectroscopy (PAS). Recent developments in the techniques themselves and in important key system components (such as sources) are considered. Finally, we have completed a survey of recent published results for the detection of a number of gas species and summarised these in Table 8 at the end of the article. The list of measurands covers ammonia, benzene, carbon dioxide, carbon monoxide, ethane, formaldehyde, hydrogen sulfide, methane, nitric oxide, nitrous oxide, nitrogen dioxide, sulfur dioxide and water vapour. From this survey we have been able to draw crosscomparisons between different approaches.

\section{Basic principles}

The fundamentals of molecular absorption spectroscopy and associated instrumental techniques have been discussed widely elsewhere ${ }^{[17,18]}$. Many chemical species exhibit strong absorption in the $\mathrm{UV} / \mathrm{visible}$, near infrared or mid infrared regions of the electromagnetic spectrum. The absorption lines or bands are specific to each species and this forms the basis for their detection and measurement. Absorption spectra in the different spectral regions have different characteristics, as shown in Table 2.

In the so-called fingerprint region of the infra-red, gas phase absorption spectra exhibit narrow lines as a result of molecular vibrations at discrete energy levels. These can be measured at high resolution, resolving the line, or at lower resolution, measuring the absorption band. Near IR spectra are typically overtones of fundamental vibrations in the mid IR and hence can be significantly weaker (eg around 100 times weaker for methane). However, the availability of high quality sources and detectors, primarily derived from telecommunications applications, can counteract this disadvantage and signal : noise ratios can be relatively high.

Table 2. Origin of absorption spectra in different regions of the electromagnetic spectrum.

\begin{tabular}{|c|c|}
\hline Spectral region & Cause of absorption \\
\hline UV $(200-400 \mathrm{~nm})$ & Electronic transitions \\
\hline Near IR $(700 \mathrm{~nm}-2.5 \mu \mathrm{m})$ & Molecular vibration \& rotation, $1^{\text {st }}$ harmonic \\
\hline Mid IR $(2.5 \mu \mathrm{m}-14 \mu \mathrm{m})$ & Molecular vibration \& rotation, fundamental \\
\hline
\end{tabular}


Optical gas detection using absorption spectroscopy is based on application of the Beer Lambert $\operatorname{Law}^{[18]}$;

$$
I=I_{0} \exp (-\alpha \ell)
$$

Where $I$ is the light transmitted through the gas cell, $I_{0}$ is the light incident on the gas cell, $\alpha$ is the absorption coefficient of the sample (typically with units of $\mathrm{cm}^{-1}$ ) and $\ell$ is the cell's optical pathlength (typically with units of $\mathrm{cm}$ ). The absorption coefficient $\alpha$ is the product of the gas concentration (for example in atm - the partial pressure in atmospheres) and the specific absorptivity of the gas $\varepsilon$ (for example in $\mathrm{cm}^{-1} \mathrm{~atm}^{-1}$ ).

Notes ${ }^{[18]}$

- In analytical chemistry and for liquid phase samples, the Beer Lambert Law is typically described using base 10 rather than $e$, with the result that quoted values of $\alpha$ are 2.3 times smaller, despite having the same apparent units. In this review, in line with most gas sensing, we use base $e$ throughout.

- The Beer-Lambert Law applies for monochromatic radiation; when using light sources that are broader than absorption lines, the width of the source must be accounted for.

- The law also assumes that there are no chemical changes in the sample - at high concentrations, dimer formation can alter spectra, but this is a minor effect for most gases at standard temperature and pressure.

For low $\alpha \ell$, equation (1) is conveniently linear with $\alpha$, as follows:

$$
\frac{\Delta I}{I_{0}} \approx \alpha \ell
$$

where $\Delta I=I_{0}-I$ and $\Delta I / I_{0}$ is the absorbance, which is unitless but often described in "absorbance units" (AU).

Limits of detection can be quantified as the noise equivalent absorbance (NEA, in AU) or the minimum detectable absorption coefficient $\left(\alpha_{\min }\right.$, in $\left.\mathrm{cm}^{-1}\right)$, allowing instrumental techniques to be compared without reference to the specific target gas. For estimates of noise and uncertainty throughout this article, we use the convention that $\Delta I$ is the root mean squared (RMS) value of intensity variations $(1 \sigma)$. For example, an NEA of $10^{-6}$ implies that for RMS changes in received light intensity at the level of 1 part in $10^{6}$, the signal : noise ratio (SNR) is unity. For many instruments, white noise dominates and therefore the SNR also depends on the measurement bandwidth $\Delta f$, as $\operatorname{SNR} \propto \Delta f^{-1 / 2}$. When operating in this domain it is therefore important for practitioners to also record the value of the measurement integration time $t$ used to obtain a certain noise limit, and / or to quote limits in units of $\mathrm{Hz}^{-1 / 2}$ or $\mathrm{cm}^{-1} \mathrm{~Hz}^{-1 / 2}$, normalising to a $1 \mathrm{~Hz}$ measurement bandwidth. As the precise conversion between $\Delta f$ and $t$ is often system - specific ${ }^{[18]}$, we have simply quoted authors' own estimates for either, or both, in this article.

Measurement of the level of absorbed light in the sample is actually proportional to the number density $\mathrm{N}$ of target molecules in the sample. To convert to more typical units of $\mathrm{ppm}$ by volume or \%volume at different temperatures $\mathrm{T}$ and pressures $\mathrm{P}$, adjustments must be made using the ideal gas equation $P V=N k_{B} T$, where $V$ is the volume of a closed cell, $k_{B}$ is the Boltzmann constant and $N$ is the number of molecules in the cell. 
An absorption spectrum is a plot of $\alpha$ or $\varepsilon$ as a function of wavelength (eg in $\mu \mathrm{m}$ ) or its reciprocal, wavenumber (in $\mathrm{cm}^{-1}$ ). Public-domain quantified spectra are available from the US National Institute of Standards and Technology (NIST) ${ }^{[19]}$, Pacific Northwest National Laboratory $(\mathrm{PNNL})^{[19]}$, and may be calculated using information in the Hitran database ${ }^{[20]}$. Typical absorption spectra are shown in Figure 1, for a series of gases in the mid IR, and in Figure 2, for a single gas (methane) plotted at higher resolution.

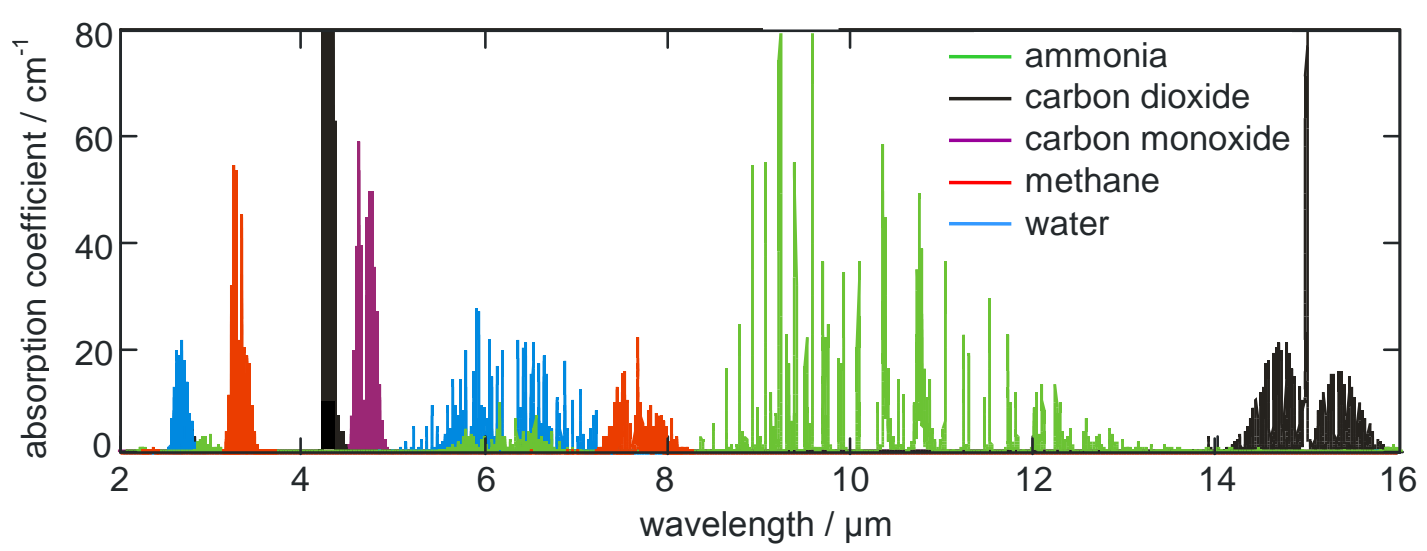

Figure 1. Absorption spectra for 5 gases in the mid IR region of the spectrum (all at $100 \%$ vol), taken from the PNNL database ${ }^{[19]}$.

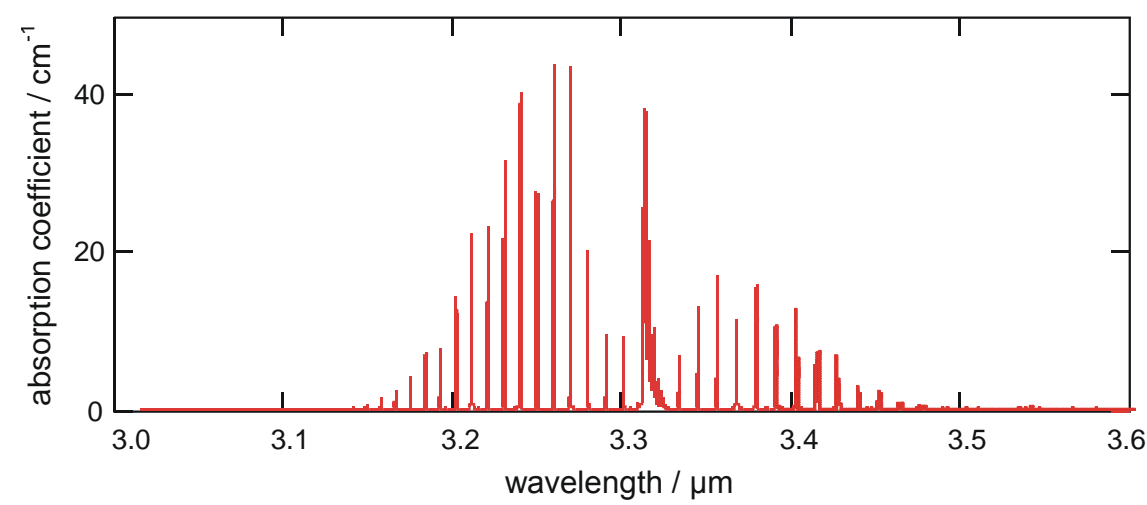

Figure 2. Expanded view of methane spectrum in the mid IR, from $3-3.6 \mu \mathrm{m}$, also taken from the PNNL database ${ }^{[19]}$.

At atmospheric pressure, a single gas line has a pressure broadened Lorentzian profile ${ }^{[18]}$, such that

$$
\alpha=C_{m o l} s \frac{\gamma}{\pi\left(\gamma^{2}+\left(v-v_{0}\right)^{2}\right)}
$$

Where $C_{m o l}$ is the gas concentration in units of molecules $\mathrm{cm}^{-3}, S$ is the line intensity $\left(\mathrm{cm}^{-1} /\right.$ molecule $\left.\mathrm{cm}^{-2}\right), y$ is the line halfwidth at half maximum $\left(\mathrm{HWHM}, \mathrm{cm}^{-1}\right), v$ is the wavenumber $\left(\mathrm{cm}^{-1}\right)$ and $v_{0}$ is the position of the line centre. Figure 3 shows an example absorption line profile for methane at $1.651 \mu \mathrm{m}$ at atmospheric pressure. Variations in the linewidth at different pressures must be accounted for, especially in high resolution measurement schemes. The linewidth can also be 
influenced by the background matrix for the gas. Typically, so-called "self-broadening" parameters (the linewidth for the gas surrounded by itself) and "air-broadening" parameters (the linewidth for small quantities of the gas in air) are well-known for commonly detected gases. For example, the Hitran database provides known halfwidths for air-broadened and self-broadened lines ( $\gamma_{\text {air }}$ and $\gamma_{\text {self }}$, respectively). At atmospheric pressure, the halfwidths are functions of both pressure and temperature. The value of $y$ for a gas at pressure $P(\mathrm{~atm})$, temperature $T(\mathrm{~K})$ and at a partial pressure $P_{\text {partial }}(\mathrm{atm})$ is given by ${ }^{[21]}$

$$
\gamma=\gamma_{\text {air }}\left(P-P_{\text {partial }}\right)+\gamma_{\text {self }} P_{\text {partial }}
$$

For significantly different matrices, linewidth measurement may be required, especially for gases that can have strong interactions with their neighbours, for example via $\mathrm{H}$-bonding in water vapour. But in any case (any matrix or pressure), the integrated area under the curve is proportional to the number density of molecules present.

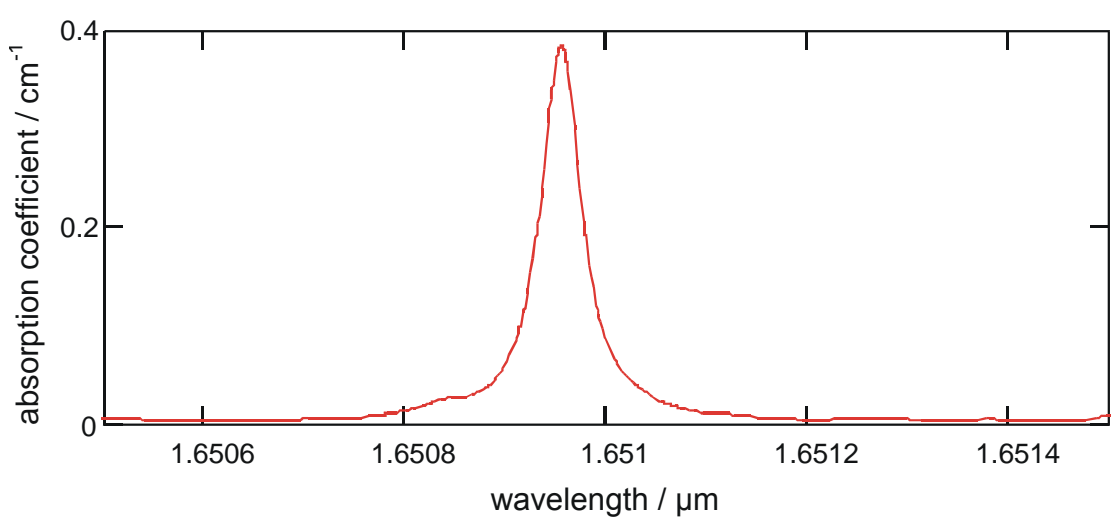

Figure 3. Absorption spectrum for $100 \%$ methane at atmospheric pressure at $1.651 \mu \mathrm{m}$, calculated from Hitran ${ }^{[20]}$. The feature actually consists of four main underlying absorption lines that cannot be resolved at atmospheric pressure.

A discussion of different technology platforms using these principles now follows, categorised as nondispersive infrared (NDIR) gas sensing (characterised by its broadband measurements), correlation spectroscopy, gas sensing using compact spectrophotometers and tunable diode laser spectroscopy (TDLS), which encompasses several different techniques. Finally, cavity-enhanced techniques and photoacoustic spectroscopy (PAS) are considered separately, both of which can be deployed in combination with other spectroscopic techniques. In each case we consider principles of operation, critical enabling technology and significant limitations.

\section{Optical gas cells}

Gas cell configurations may be altered to suit a wide variety of applications, as follows.

- Use of long path cells, to increase the magnitude of the signal according to equation (2).

- Use of optical fibre to deliver light to a sample cell in a remote location

- Use of hollow core optical fibre to form a long, thin gas cell. 
Coupling light into many of these cells is facilitated by the use of lasers, as a significantly greater proportion of the light may be collimated in a narrow beam and / or launched into optical fibre than would be the case for broadband sources. Each configuration is now discussed in turn. Cell configurations that are specific to a particular spectroscopic technique are introduced in later sections, and cavity-enhanced techniques have their own section at the end of this review.

Gas cells are not simply passive elements of a system; their design can be a significant contributor to instrument uncertainty. When used at high spectral resolution, for example with spectrophotometers or tunable diode lasers, gas cells can form low finesse Fabry-Perot etalons that give rise to unwanted optical interference fringes, especially when using high coherence, laser sources ${ }^{[22]}$. These fringes can be the limiting source of uncertainty as they are liable to drift with temperature changes and can be indistinguishable from the spectral signature of the gas absorption lines. Because gas lines can be inherently narrow, interference fringes can also be a significant performance-limiting factor in broadband absorption measurements ${ }^{[23]}$.

\subsection{Long path gas cells}

Gas cells with the highest sensitivity often employ multipass cells with pathlengths from tens of metres (multipass cells such as those devised by Herriott ${ }^{[2]}$, White ${ }^{[25]}$ and Chernin ${ }^{[26]}$ ) to $\mathrm{km}$ (using so-called cavity-enhanced and ringdown techniques). The latter are covered separately in section 7 because of the volume of recent research in both fields, and because it is helpful first to introduce the techniques of NDIR, spectrophotometry and TDLS.

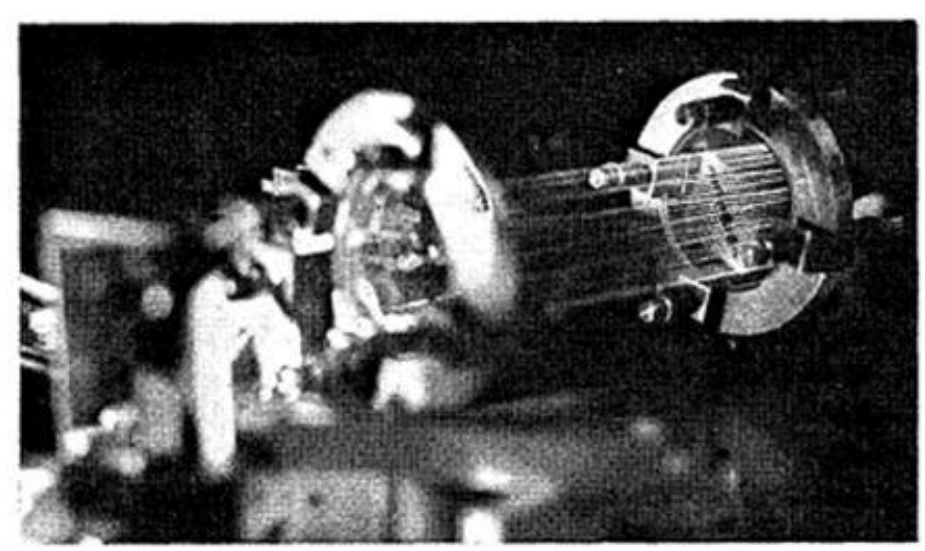

Figure 4. Early Herriott cell, using smoke to visualise the reflected beams between concave mirrors.

Taken from [27].

The most commonly used multipass cell is of the Herriott type, either in its standard configuration (as shown in Figure 4) or as an astigmatic variant ${ }^{[28]}$. Advantages of the latter are that the beam spot pattern at the mirrors is distributed over the entire mirror surface, giving better separation between individual spots, and that longer pathlengths can be achieved for a given cell volume. Although Herriott cells require time-consuming alignment, systems have been made that are field robust and even sufficiently lightweight for deployment with a weather balloon ${ }^{[29]}$ (see Figure 5). A commercial, portable instrument has also recently been introduced using a Herriott cell to give a limit of detection of $1 \mathrm{ppm}$ methane ${ }^{[30]}$. When using a Herriott cell, one might expect the improvement in SNR to scale with the 
pathlength enhancement. However, additional fringes arise as a result of interference between successive passes across the cell, and this can result for example in an order of magnitude deterioration in the $\mathrm{SNR}^{[31]}$.

Various alternative approaches have been demonstrated in recent years; a number are compared in Table 3. The superior pathlength : volume ratio of the astigmatic Herriott cell, together with the stability derived from its parent the standard Herriott cell, make it stand out and probably account for its popularity. The Chernin cell is claimed to have a number of advantages including the acceptance of high NA beams, however has been little used outside of research labs. The best pathlength : volume ratio exists for hollow core optical fibres, hence the considerable recent effort to develop practical systems using this technology (see 3.3 for further details). Other systems worthy of note include the circular multireflection cell of Ofner et al ${ }^{[32]}$ with a diameter of $6 \mathrm{~cm}$, giving 17.5 beam passes and an equivalent pathlength of up to $1.05 \mathrm{~m}^{[32]}$. This was coupled to an FTIR spectrometer and used to measure $\mathrm{CO}_{2}$ at $4.2 \mu \mathrm{m}$.

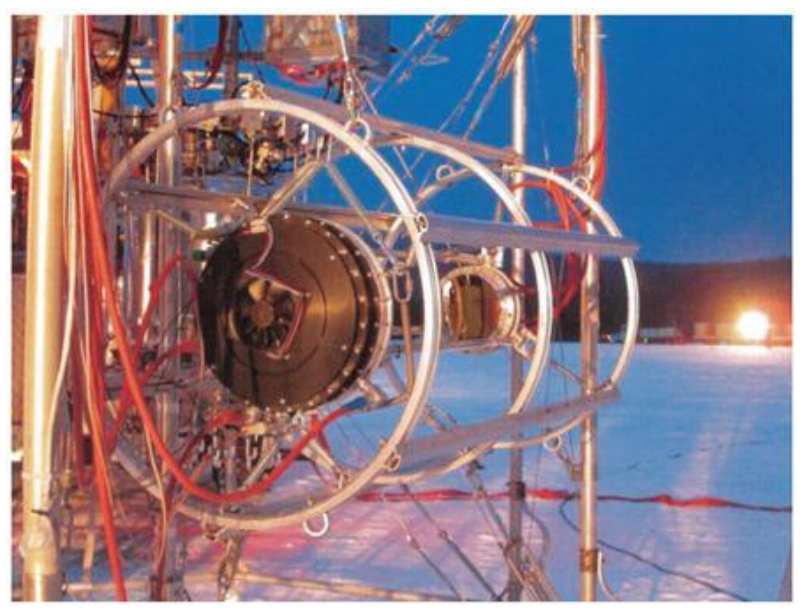

Figure 5. Herriott cell included in a weather balloon payload, used at an altitude of $32,000 \mathrm{~m}$ to measure methane and water vapour with pathlengths of $74 \mathrm{~m}$ and $36 \mathrm{~m}$ respectively. Taken from [29].

Finally, although the integrating sphere cannot offer the optical pathlength of the best of its rival multipass cells, it nevertheless has a significant advantage in its tolerance to misalignment. Integrating spheres are formed by taking a material with high diffuse reflectivity (typically barium sulfate, poly[tetrafluoroethylene] PTFE, or a gold coated roughened surface, depending on the spectral region of operation) and wrapping it around on itself to form a spherical cavity ${ }^{[41]}$ (see Figure 6). Hodgkinson et al have analysed the distribution of optical pathlengths in these devices and shown that the resulting apparent deviation from Beer's law (equation (1)) is predictable ${ }^{[33]}$. Hawe et al have detected $\mathrm{NO}_{2}$ and $\mathrm{SO}_{2}$ in the 200-600 nm region using a $5 \mathrm{~cm}$ internal diameter integrating sphere coupled to a broadband source and a UV-visible spectrophotometer using multimode fibre ${ }^{[34]}$. The mean internal pathlength of the sphere depends on its reflectivity; for $\mathrm{SO}_{2}$ at $280 \mathrm{~nm}$ this was $41 \mathrm{~cm}$ and for $\mathrm{NO}_{2}$ at $370 \mathrm{~nm}$ it was $55 \mathrm{~cm}$. Detection limits for $\mathrm{NO}_{2}$ and $\mathrm{SO}_{2}$ were $4 \mathrm{ppm}$ and $11 \mathrm{ppm}$ respectively. Interestingly, the mean pathlengths are somewhat lower than for the specularly reflective cell of Ofner et al above, which had a similar internal diameter. This was presumably caused by the relatively low reflectivity of the integrating sphere in the UV region. 


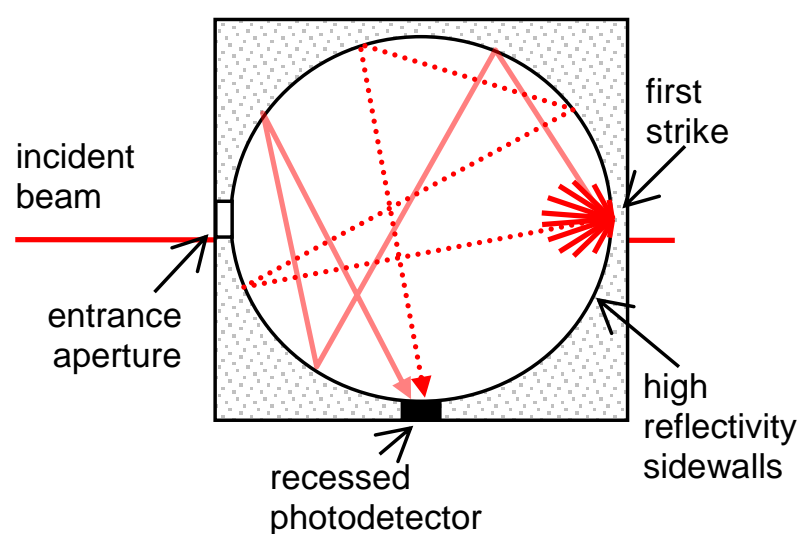

(a)

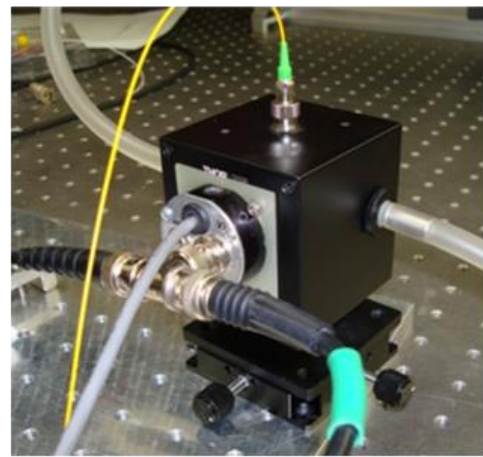

(b)

Figure 6. (a) Simplified model of an integrating sphere, showing a collimated beam making a first pass across the cell to the first strike spot and two examples of subsequent random passes across the cell. Adapted from [35]. (b) Commercially available integrating sphere (Thorlabs IS200-4) in use as a gas cell.

Table 3. Comparison of different approaches to multipass cells used in TDLS (one example given of each type). Figures in italics are the authors' estimates based on information provided in references.

\begin{tabular}{|c|c|c|c|c|c|c|}
\hline Approach & Advantage & $\frac{\text { Pathlength }}{1 \mathrm{~m}}$ & $\begin{array}{l}\text { Number } \\
\text { of passes }\end{array}$ & $\frac{\text { Volume }}{\text { / litres }}$ & $\frac{\text { Pathlength / }}{\frac{\text { volume }}{110^{3} \mathrm{~m}^{-2}}}$ & $\underline{\text { Ref }}$ \\
\hline Herriott cell & $\begin{array}{l}\text { Optomechanically stable; } \\
\text { simple pathlength changes }\end{array}$ & 30 & 74 & 1 & 30 & [36] \\
\hline $\begin{array}{l}\text { Astigmatic Herriott } \\
\text { cell }\end{array}$ & Very long optical paths & 36 & 182 & 0.3 & 120 & [28] \\
\hline White cell & Accepts high NA beams & 7.5 & 12 & 10 & 0.75 & [25] \\
\hline $\begin{array}{l}\text { White cell with } \\
\text { recirculations }\end{array}$ & $\begin{array}{l}\text { Accepts high NA beams; } \\
\text { gives longer pathlengths }\end{array}$ & 5984 & 272 & $\begin{array}{c}\text { Not } \\
\text { stated }\end{array}$ & - & [37] \\
\hline Chernin cell & $\begin{array}{l}\text { High NA beams; compact; } \\
\text { pathlength changes possible }\end{array}$ & $\sim 1500$ & $\sim 500$ & 160 & 9 & [38] \\
\hline $\begin{array}{l}\text { Combination cell } \\
\text { with } 3 \text { mirrors }\end{array}$ & $\begin{array}{l}\text { Uses standard mirrors; } \\
\text { stable; simple set-up }\end{array}$ & 140 & 130 & 5.4 & 26 & [39] \\
\hline $\begin{array}{l}\text { Twisted cylindrical } \\
\text { mirrors }\end{array}$ & $\begin{array}{l}\text { Pathlength alteration via } \\
\text { twist angle }\end{array}$ & 58 & 49 & 5.4 & 11 & [40] \\
\hline $\begin{array}{l}\text { Circular multi- } \\
\text { reflection cell }\end{array}$ & $\begin{array}{l}\text { Pathlength alteration from } \\
\text { outside the cell; very simple } \\
\text { cylindrical optic }\end{array}$ & 1.04 & 17.5 & 0.085 & 12 & [32] \\
\hline Integrating sphere & $\begin{array}{l}\text { Simple alignment; } \\
\text { misalignment tolerant }\end{array}$ & 4.4 & 65 & 0.5 & 9 & [41] \\
\hline $\begin{array}{l}\text { Hollow core fibre } \\
\text { (see section 3.3) }\end{array}$ & Very small sample volume & 27 & 1 & $3 \times 10^{-6}$ & $8 \times 10^{6}$ & [42] \\
\hline
\end{tabular}




\subsection{Gas cells linked via optical fibre}

Optical fibre can take many forms. Common to most is that light is guided in a core region, typically with the core having a higher refractive index than the surrounding cladding, setting up the right conditions for total internal reflection for light incident over a given range of angles. Fibres may be multimode, with each of the modes having a different effective pathlength, or singlemode. Conventional multimode telecommunications fibre employs a core diameter of 50 or $60 \mu \mathrm{m}$ within a $125 \mu \mathrm{m}$ cladding, whereas the core diameter for singlemode fibre may be of the order of $5-10 \mu \mathrm{m}$, depending on the wavelength. When coherent light or very high resolution spectroscopy is employed, interference between the modes in multimode fibre can cause undesirable effects on the signal (usually termed "mode noise"), therefore singlemode fibre may be preferred.

Silica based fibres are widely used for optical telecommunications, with high transmission in the 1.530$1.565 \mu \mathrm{m}$ ("C band"), 1.260-1.360 $\mu \mathrm{m}$ ("O band") and 800-900 nm windows. The availability of erbium (Er) doped fibre amplifiers (EDFAs) has prompted rapid development in the $\mathrm{C}$ band, and many components are available that cover this region, with some extending into neighbouring $S$ and $L$ bands (1.460-1.530 $\mu \mathrm{m}$ and $1.565-1.625 \mu \mathrm{m}$ respectively). Without amplification, communication over up to many $\mathrm{km}$ of fibre is possible at data rates up to tens of Gbit/s. Fortunately, gas detection applications typically demand shorter lengths of fibre and can often accommodate greater optical losses and lower data rates, such that standard telecommunications grade optical fibre may be used over a wider range of wavelengths to cover many gas absorption lines outside the narrow telecommunications bands.

Conventional telecommunications fibres are fabricated by a drawing technique from low loss silica incorporating different dopants. The drawing technique provides an excellent surface finish, in particular at the junction between core and cladding, such that scattering losses at this interface are very low, and centering of the core is achieved to a high precision. A number of specialist fibres has also been developed to provide a wider range of transmission wavelengths, most notably in the mid IR. For example, chalcogenides have been used to link gas cells at $4.2 \mu \mathrm{m}$ for $\mathrm{CO}_{2}$ sensing in areas with difficult access such as vehicle exhausts ${ }^{[43]}$.

Often, optical fibre is simply used as a conduit to a more conventional, remote gas cell, for a number of reasons ${ }^{[44]}$. The first is that multiplexing of multiple gas cells allows the relatively high cost of certain light sources (such as tunable diode lasers) to be shared, bringing the cost per sensor head to a level where it can compete with traditional installed sensors. The second is that gas detection is often required in potentially explosive areas where intrinsic safety (compliance with the Explosive Atmospheres or ATEX directive in the $\mathrm{EU}^{[45]}$ ) may be required. It is often simpler to ensure the intrinsic safety of a fibre optically linked sensor than that of a conventional, electrically addressed system, for example for continuous wave $(\mathrm{CW})$ sources by ensuring that either the light power remains below $35 \mathrm{~mW}$ or the light intensity remains below $5 \mathrm{~mW} \mathrm{~mm}^{-2}[46]$.

Interaction between the light guided by the fibre and the measurand can be achieved by a variety of means, as reviewed by Waechter et al ${ }^{[47]}$ and illustrated in Figure 7 . For those involving evanescent fields, the proportion of light interacting with the surrounding measurand is a function of the latter's refractive index, therefore these techniques have more commonly been used with higher index liquid samples. However, there are a number of examples of their use for gas detection.

A variety of multiplexing schemes has been developed to link a large number of sensor heads, each of which samples the gas concentration at a different point. Stewart et al developed a switched system in 
which each of the 64 sensor heads shared a single laser source but had a dedicated delivery and return fibre with detector ${ }^{[48]}$. Ho et al have used a single pulsed laser source and detector, with cells arranged in parallel and optical fibre delay lines in-between at each stage. Individual gas cells were then identified by time gating the source pulses ${ }^{[49]}$. Attempts to multiplex more than one sensor head per fibre have included deliberate use of etalons created by gas cells of unequal length in a ladder topology ${ }^{[50]}$, and frequency shift interferometry ${ }^{[51]}$, a technique formerly used to interrogate optical fibre Bragg gratings. The latter method had the advantage of being capable of dealing with multiple gas absorption lines (including the possibility of spectral overlap) as well as multiple sensor locations (up to 17). Nevertheless, the additional complexities of optical fibre systems compared with free space systems often mean that limits of detection are compromised by residual interference fringes from etalons formed by gas cells, connectors and joints in the fibre ${ }^{[52]}$.

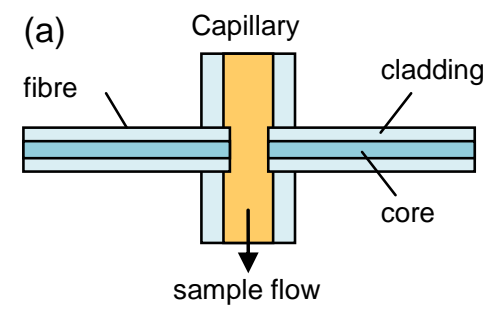

(c)

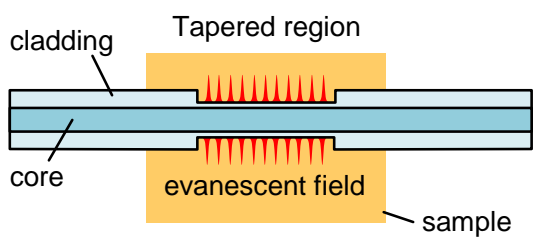

(e)

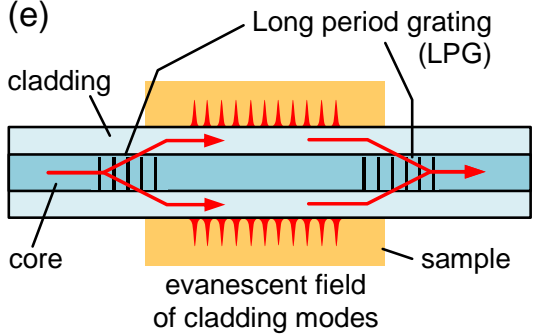

(b)

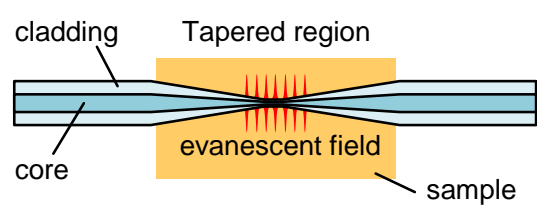

(d)

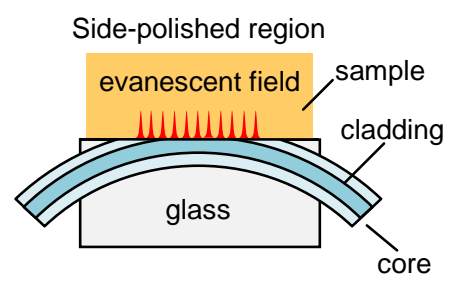

(f)

Diffusion region

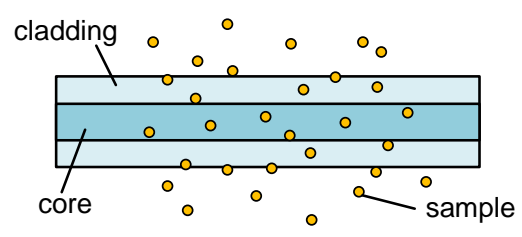

Figure 7. Techniques providing interaction between light guided within an optical fibre and an external measurand: (a) light coupled out of the fibre and back in; evanescent fields in (b) a tapered region, (c) an etched region, (d) a side-polished region or (e) using long period gratings to couple light in and out of cladding modes; (f) diffusion of some gases $\left(\mathrm{eg} \mathrm{H}_{2}\right)$ into the fibre core. Modified from [47]. 


\subsection{Use of microstructured optical fibre}

Photonic crystal fibre (PCF) was originally developed for photonic and telecommunications applications ${ }^{[53]}$. A periodic refractive index within the fibre (typically generated by an array of holes that pass along the length) forms a photonic crystal, the periodicity of the refractive index in the photonic crystal being analogous to the periodicity of electronic energy levels in more conventional ionic crystals. A photonic quantum well is formed that confines the light modes more effectively than in conventional fibre, such that, in principle, photonic crystal fibres of the right lattice spacing and hole size can maintain singlemode operation over almost their entire transmission range ${ }^{[53]}$. A further advantage is a lower effective index, reducing Fresnel reflections at the fibre ends.

It was quickly realised that the holes in these fibres can act as convenient repositories for gases, and that a high degree of overlap between the physical location of the holes and the guided modes results in strong interaction between the two, and thus the potential for use in gas sensing. The greatest levels of overlap (up to $98 \%$ ) are found with hollow core fibres ${ }^{[54]}$, for which the range of operation is more restricted. A photonic bandgap, and therefore waveguiding, only occurs in particular wavelength bands. Solid core fibres can also be used where the core is small and the guided mode extends into the surrounding voids, ideally with very thin supporting structures ${ }^{[55]}$ in so-called suspended core fibres, in which the level of mode / air overlap is small for gas phase sensing. Lehmann et al have compared both types and concluded that hollow core fibres are better suited to trace gas detection, whereas suspended core fibres, having lower losses, might be suited to higher gas concentrations, longer length operation, or even distributed sensing of gases ${ }^{[56]}$. Figure 8 shows examples of microstructured optical fibres used in chemical sensing. Most examples are formed from air / silica, however microstructured fibres for mid IR transmission have been fabricated from both chalcogenides ${ }^{[57,58]}$ and silver halides ${ }^{[59]}$. Singlemode microstructured fibre has been demonstrated by Shephard et al for 3.1-3.3 $\mu \mathrm{m}$ operation using a $40 \mu \mathrm{m}$ core diameter photonic bandgap fibre (PBGF) fabricated from silica ${ }^{[60]}$. 


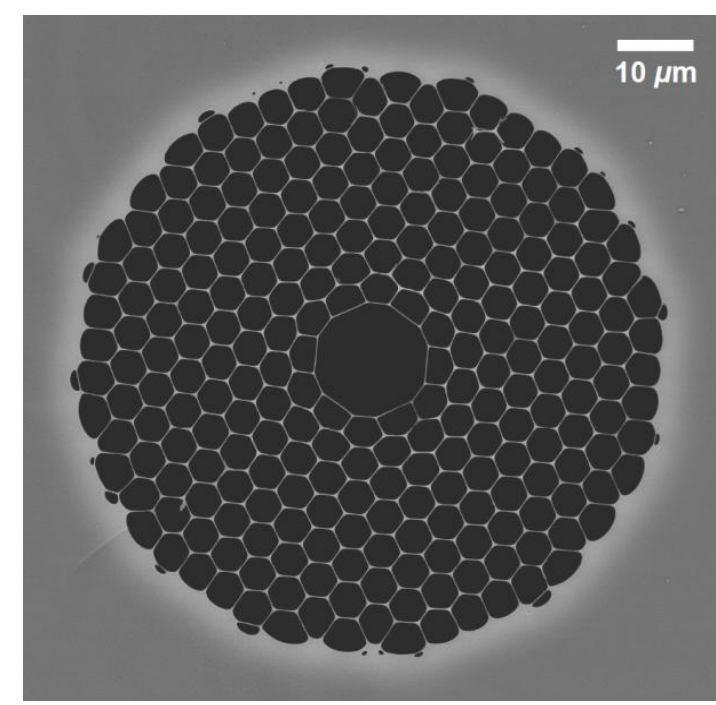

(a)

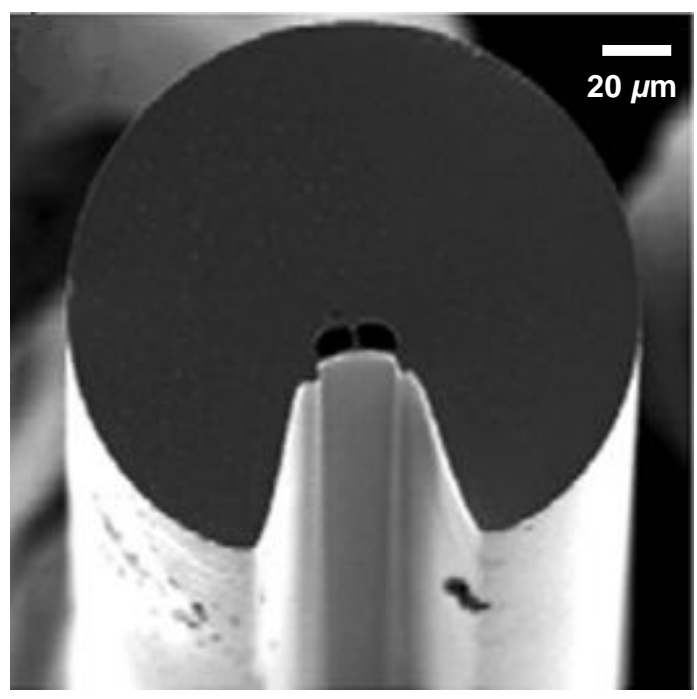

(c)

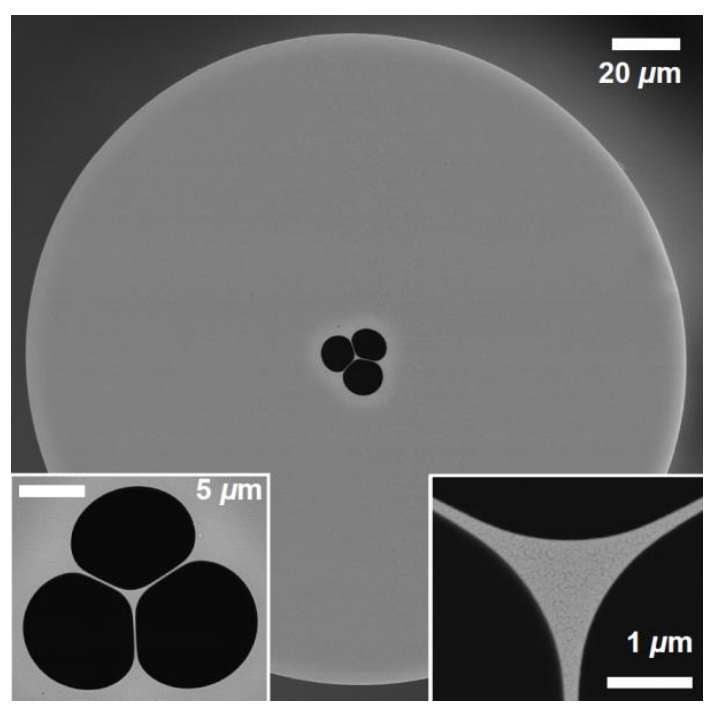

(b)

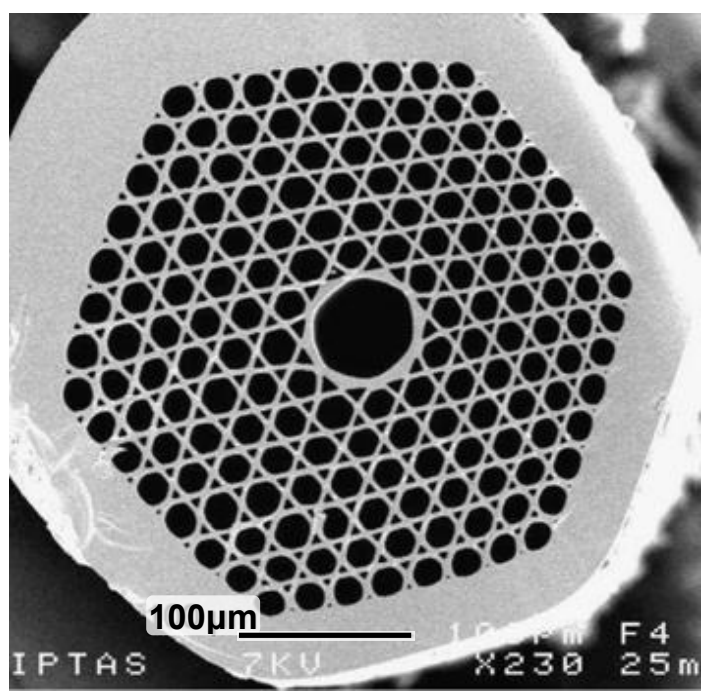

(d)

Figure 8. Microstructured fibres suitable for chemical sensing. (a) Air-silica hollow core PBGF and (b) suspended core holey fibre, both from [61]. (c) Modified suspended core fibre with slot, used for distributed fluorescence sensing, from [55]. (d) Chalcogenide hollow core PCF, from [62].

\subsubsection{Hollow core waveguides}

Interest has been strong in the use of hollow core waveguides as a long pathlength, low volume gas cell. Sub-nanolitre sample volumes have even been demonstrated for gas sensing ${ }^{[63]}$. Light is confined in a narrow capillary structure by a series of internal reflections at high reflectivity walls. Following the same conventions as the majority of the literature, we term these structures hollow core waveguides (HCWG), in contrast to hollow core photonic bandgap fibres (HC-PBGF) described above. Losses can be consequently high, but can generally be accommodated over distances of several metres without significantly affecting sensor performance. The attenuation in hollow core waveguides varies with inner core radius $a$ and bend radius $r$ as $1 / a^{3}$ and $1 / r^{[67]}$; typical figures are shown in Figure 9. 


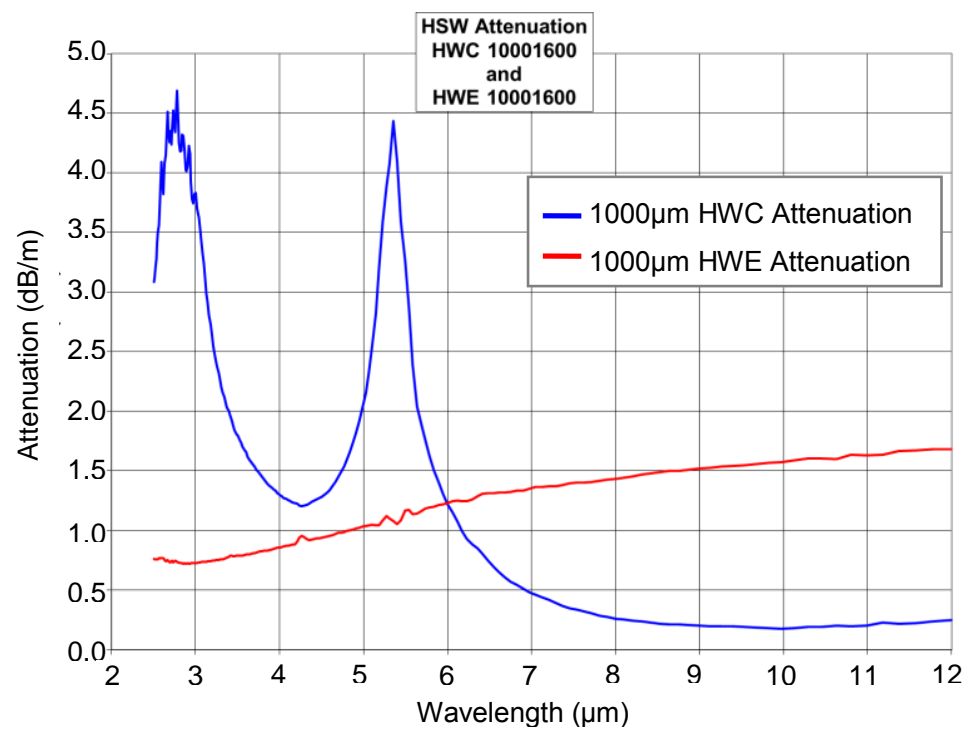

(a)

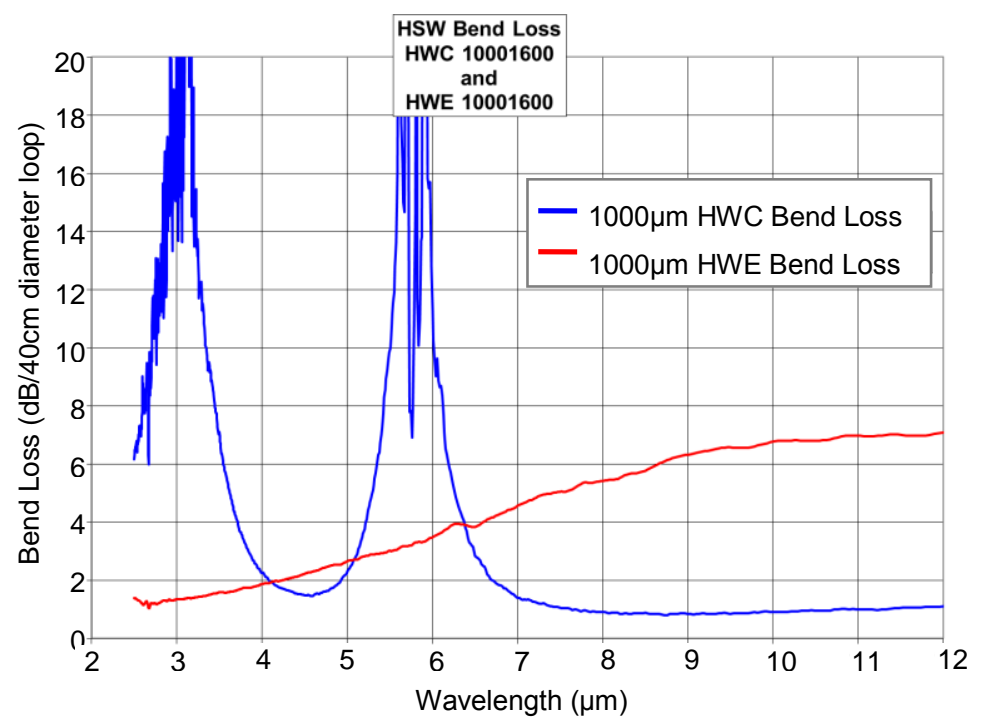

(b)

Figure 9. Measured losses for commercially available hollow core waveguides: type HWC (used for $\mathrm{CO}_{2}$ laser beam delivery) and type HWE (used for Er:YAG laser beam delivery). (a) Spectral loss for straight waveguide, (b) bend losses for a $40 \mathrm{~cm}$ radius, $360^{\circ}$ bend. Reproduced with permission of Polymicro Technologies, a subsidiary of Molex Inc.

\subsubsection{Performance limitations}

Kim et al have used a hollow core waveguide with an internal IR reflective coating consisting of $\mathrm{Ag}$ with a protective $\mathrm{Agl} \mathrm{layer}{ }^{[64]}$. A $1 \mathrm{~m}$ length of fibre with internal diameter $750 \mu \mathrm{m}$ was coupled to a conventional laboratory FTIR spectrometer and used to measure absorption in the wavenumber range $3000-600 \mathrm{~cm}^{-1}(3.3-17 \mu \mathrm{m})$. Limits of detection were $16 \mathrm{ppb}$ for $\mathrm{CO}_{2}$ at $4.2 \mu \mathrm{m}$ and $520 \mathrm{ppb}$ for $\mathrm{CH}_{4}$ at $7.7 \mu \mathrm{m}$. The feasibility of using the same design of waveguide for spectral measurements in the UV $(180-205 \mathrm{~nm})$ has also been reported ${ }^{[65]}$. 
Charlton et al have coupled the output from a $10.3 \mu \mathrm{m}$ quantum cascade laser (QCL, see section 6.3) into a $700 \mu \mathrm{m}$ core diameter capillary of this type, for the purpose of detecting ethyl chloride ${ }^{[66]}$. They used a cell length of $4 \mathrm{~m}$ and $\mathrm{HgCdTe}$ detector to achieve a detection limit of $0.5 \mathrm{ppm}$, but the noise equivalent absorbance (NEA) was not stated. Using the system shown in Figure 10, Fetzer et al reported an NEA of $1.2 \times 10^{-5}$ with a hollow core waveguide length of $3 \mathrm{~m}$, operating in the near IR at $1.5 \mu \mathrm{m}^{[67]}$. However, drift over a period of 4 hours (involving a $10^{\circ} \mathrm{C}$ change in room temperature) increased the NEA to $6 \times 10^{-5}$.
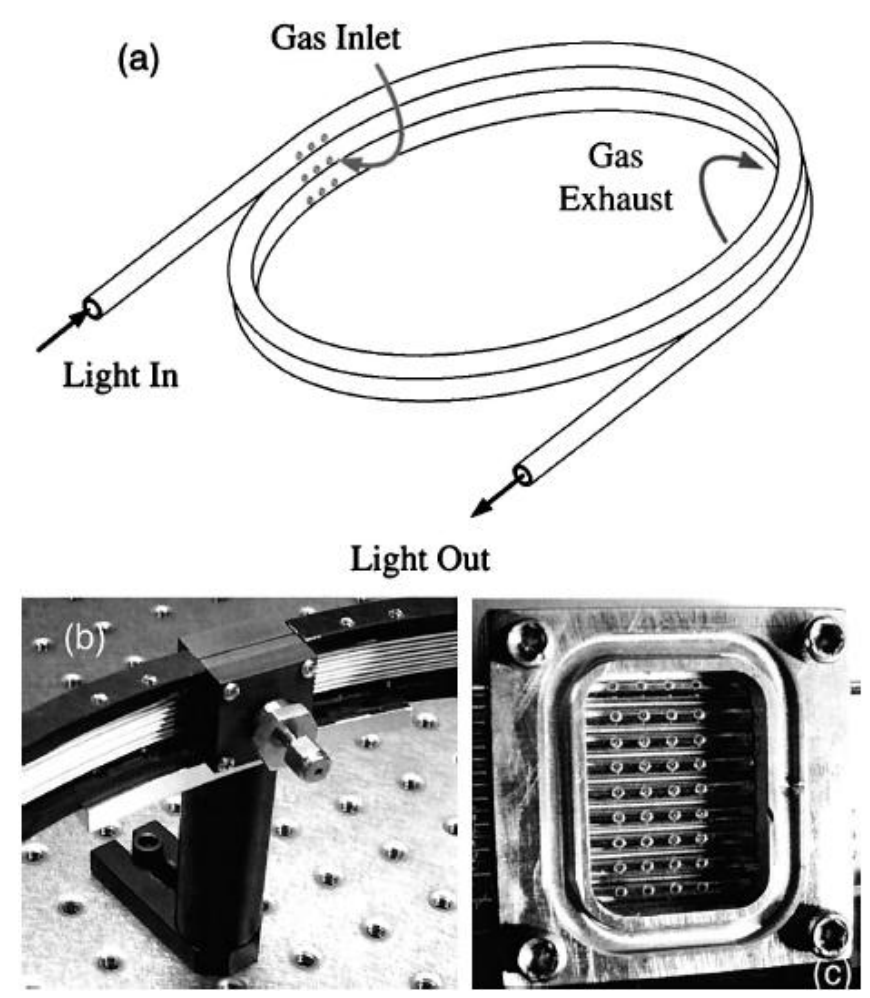

Figure 10. Construction of a low volume, fast $(6 \mathrm{~s})$ response gas cell using a coiled hollow core waveguide. (a) Schematic diagram, (b) photograph of inlet manifold, (c) close-up of inlet manifold showing closely spaced, $50 \mu \mathrm{m}$ diameter drilled holes. Taken from [67].

There has been a limited number of studies on the fundamental performance limitations of gas sensing using microstructured fibres. Gensty et al reported that the relative intensity noise (RIN) of a quantum cascade laser, measured at $50 \mathrm{MHz}$, was consistently lowered (by a factor of around $4 \mathrm{~dB}$ ) by being transmitted through a hollow core fibre; they attributed this to a filtering effect of the waveguide ${ }^{[68]}$.

Parry et al have reported spectral artefacts when using a $10 \mu \mathrm{m}$ core PBGF operated at a wavelength of $1.650 \mu \mathrm{m}$ for methane detection ${ }^{[69]}$. They attributed this to two causes, (i) interference caused by backreflections from two ends of the fibre, and (ii) interference between core and cladding modes propagating in the fibre. The latter effect dominated, but could be minimised by placing detectors or coupling optics as close as possible to the end of the PBGF, minimising the launch and collection of cladding modes. Interferences fringes were reduced by thermally cycling the fibre using resistive heating elements and averaging signals over 35 minutes, or by subtraction of a zero reference within a short period of time. The resulting limit of detection was reported to be $500 \mathrm{ppm}$ acetylene at $1.519 \mu \mathrm{m}$ for operation at 70 torr. 
Finally, Chen et al have investigated the mode noise limitation of using hollow core waveguides whose diameter is a large multiple of the operating wavelength $(750 \mu \mathrm{m} \text { diameter operated at } 1.68 \mu \mathrm{m})^{[70]}$. In this system the level of mode noise amounted to $\Delta \mathrm{I} / \mathrm{I} \sim 10^{-4}$. This was reduced for (i) straight fibres rather than bent fibres, (ii) locating the detector as close as possible to the end of the fibre, so as to collect a larger number of modes, and finally by vibrating the fibre at a frequency much greater than the measurement bandwidth, so as to average over a larger number of modes. Using the latter technique with a bent fibre, the authors were able to demonstrate an NEA of $\Delta \mathrm{I} / \mathrm{I} \sim 10^{-5}$.

\subsubsection{Practical matters}

Photonic crystal fibres and other PBGFs can be cleaved using standard equipment and spliced to each other and to conventional fibre ${ }^{[53]}$. Endface distortion is possible if the air filling fraction is high $(>50 \%)^{[53]}$, as demonstrated in the work of Benabid et al who cleaved and spliced a PBGF to create a stable gas cell ${ }^{[71]}$. By recleaving the joint after splicing, they were able to show the potential for surface tension to create a concave cavity in this case, shown in Figure 11.

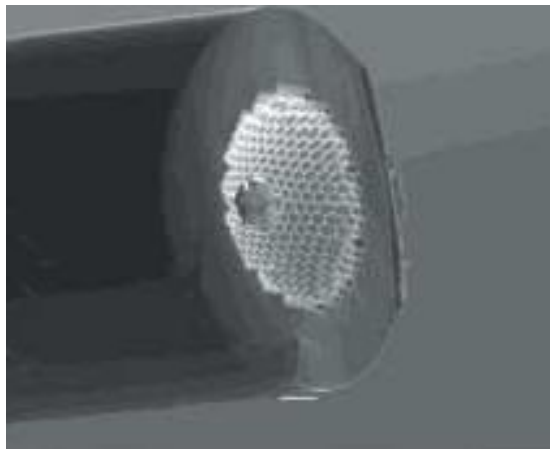

(a)

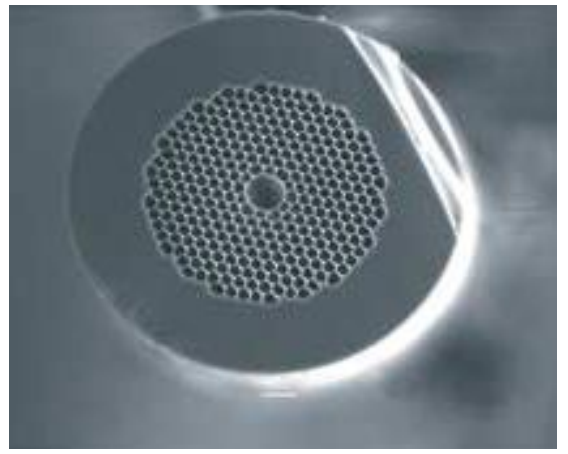

(b)

Figure 11. SEM images of a joint between PBGF and standard singlemode fibre that was spliced and then recleaved for examination: (a) at the joint, showing a concave cavity formed by surface tension during splicing, (b) a few $\mathrm{mm}$ back from the joint, showing that the integrity of the structure was maintained at that point. Taken from [71].

One of the main engineering difficulties of using gas cells with such a high aspect ratio is the long time taken to fill them with the target gas ${ }^{[72]}$. Techniques to improve the sample filling time include increasing the pressure difference across the fibre to drive the gas through, increasing the cell diameter (at the expense of using a larger volume cell) or introducing holes to allow gas flow or diffusion along the waveguide's length, reducing the effective value of $\ell$. A number of these is compared in Table 4.

Ma et al have used a porous capillary waveguide as a means of reducing the sample retention time (and therefore the instrument response) while retaining its high aspect ratio (length $17 \mathrm{~cm}$, diameter $170 \mu \mathrm{m})^{[73]}$. The pore size, of between several to tens of $\mathrm{nm}$, was sufficiently large to allow gas diffusion while being sufficiently small to cause little perturbation to the transmitted light, with transmission losses estimated to be $0.5 \mathrm{~dB}$ over $16 \mathrm{~cm}$ (equivalent to $3.1 \mathrm{~dB} / \mathrm{m}$ ) in the $1.550 \mu \mathrm{m}$ region. A 1/e response time for gas diffusion of $<5 \mathrm{~s}$ was measured, compared with response times of the order of minutes for similar dimensions of air clad or hollow core waveguides. 
A number of authors have drilled holes into the sides of their fibres to facilitate either diffusion - limited or pumped gas sampling (see Table 4). Holes can be drilled to a high tolerance using femtosecond lasers and have the advantage of being convenient to use once in place. However, the estimated loss for a drilled hole $\left(0.35 \mathrm{~dB}\right.$ for a $1.5 \mu \mathrm{m}$ diameter hole $\left.{ }^{[74]}\right)$ is greater than that for similarly sized gaps between butt coupled sections $\left(0.044 \mathrm{~dB}^{[75]}\right)$. Figure 12 shows an SEM image of a drilled hole.

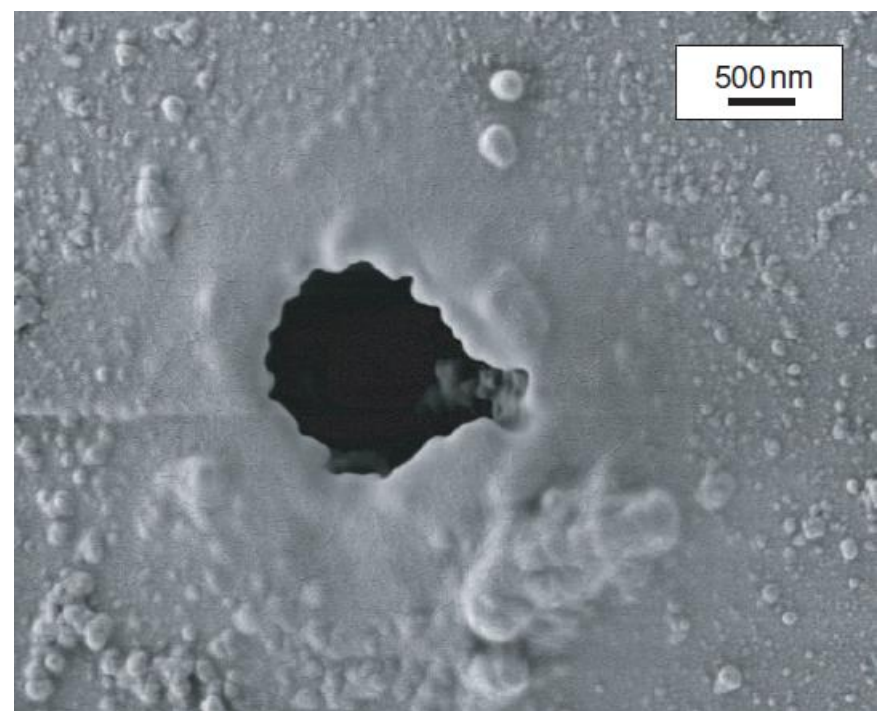

Figure 12. SEM image of a femtosecond laser-drilled hole through the side of a PBGF. Taken from [74].

Hensley has reported in detail the method used to micromachine a series of holes in a $\mathrm{PBGF}^{[74]}$. A femtosecond laser (90 fs pulses of $1 \mathrm{~mJ}, 1 \mathrm{kHz}$ repetition) was used to drill holes of approx $1 \mu \mathrm{m}$ diameter into the fibre. To avoid optical aberrations in the drilling beam and maintain beam quality both inside and outside the fibre, refractive index matching fluid was used as shown in Figure 13. Slowly pumping the fluid through the fibre during the drilling process also had the benefit of removing debris. The fluid was subsequently removed by flushing the fibre with methanol and nitrogen.
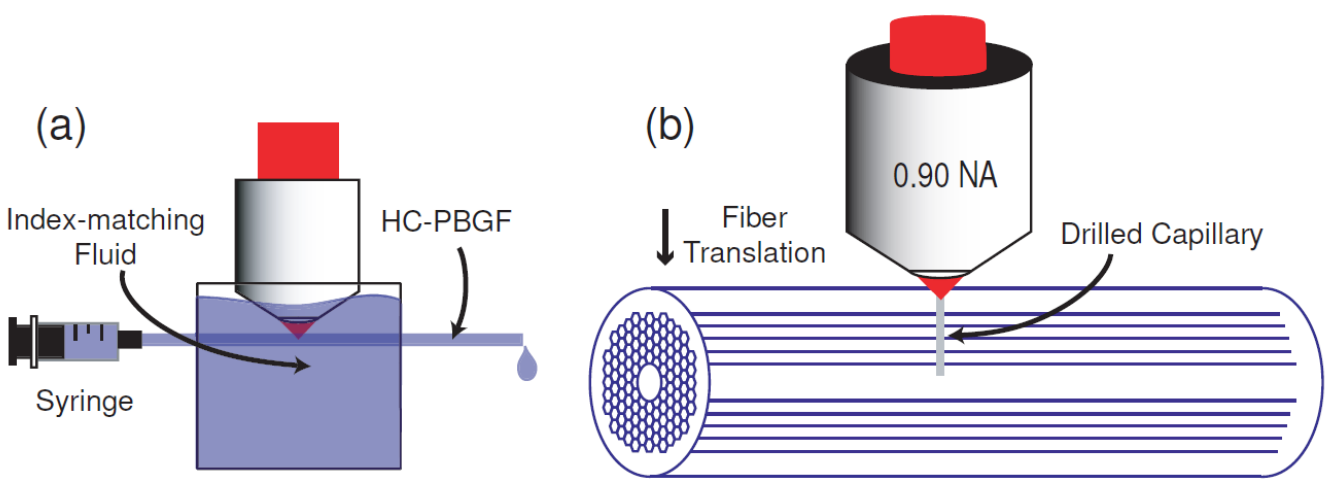

Figure 13. Femtosecond hole-drilling process using refractive index matching fluid and a high numerical aperture (NA) lens. Taken from [74]. 
Table 4. Different approaches to improving sampling response times for hollow core fibres. Figures in italics have been estimated by the authors on the basis of reported details.

\begin{tabular}{|c|c|c|c|c|c|c|c|}
\hline Technique & $\frac{\frac{\text { Applied }}{\text { pressure }}}{\text { / Pa }}$ & $\frac{\frac{\text { Inner }}{\text { diameter }}}{\underline{\mu \mathrm{m}}}$ & $\frac{\frac{\text { Total }}{\text { length }}}{\underline{\mathrm{m}}}$ & $\frac{\frac{\text { A: Sample }}{\text { volume / }}}{\underline{\mathrm{mm}^{3}}}$ & $\begin{array}{l}\text { B: Sample } \\
\text { filling time }\end{array}$ & $\frac{\text { Effective flow }}{\frac{\text { rate }(\mathrm{A} / \mathrm{B})}{1 \mathrm{~cm}^{3} \mathrm{~min}^{-1}}}$ & $\underline{\text { Ref }}$ \\
\hline Increased bore & 0 & 1000 & 0.2 & 160 & $0.05-0.1 \mathrm{~s}$ & $190-100$ & [76] \\
\hline $\begin{array}{l}\text { Holes formed between } 4 \\
\text { butt coupled sections }\end{array}$ & 0 & 16 & 0.48 & 0.01 & $\begin{array}{l}250 \mathrm{~s} \\
\text { (time to } 95 \% \\
\text { response) }\end{array}$ & $2.4 \times 10^{-6}$ & [77] \\
\hline $\begin{array}{l}10 \mu \mathrm{m} \text { diameter hole } \\
\text { every } 10 \mathrm{~cm}\end{array}$ & 0 & 10.5 & 0.07 & 0.006 & $3 \mathrm{~s}$ & $1.2 \times 10^{-4}$ & [78] \\
\hline $\begin{array}{l}\text { Porous material (pore } \\
\text { size tens of } n m \text { ) }\end{array}$ & 0 & 170 & 0.17 & 4 & $<5 s$ & 0.05 & [73] \\
\hline $\begin{array}{l}\text { Pressure driven across } \\
\text { whole length }\end{array}$ & $10^{5}$ & 12.5 & $\begin{array}{l}0.3 \\
0.7 \\
2.7\end{array}$ & $\begin{array}{l}0.04 \\
0.09 \\
3\end{array}$ & $\begin{array}{l}6 \mathrm{~s} \\
28 \mathrm{~s} \\
3 \mathrm{hrs}\end{array}$ & $\begin{array}{l}4 \times 10^{-4} \\
2 \times 10^{-4} \\
1.5 \times 10^{-5}\end{array}$ & [42] \\
\hline $\begin{array}{l}50 \mu \mathrm{m} \text { gap between butt } \\
\text { coupled fibre ends, } \\
\text { vacuum applied }\end{array}$ & $10^{5}$ & 10 & 2 & 0.16 & $4 \mathrm{~s}$ & $2.4 \times 10^{-3}$ & [54] \\
\hline $\begin{array}{l}\text { Series of } 50 \mu \mathrm{m} \\
\text { diameter holes drilled } \\
\text { approx every } 15 \mathrm{~cm} \text { (see } \\
\text { Figure 10); pumped flow }\end{array}$ & $\begin{array}{l}\text { Not } \\
\text { stated } \\
\text { (small } \\
\text { pump) }\end{array}$ & 1000 & 3 & 2.4 & $6 s$ & 0.024 & [67] \\
\hline
\end{tabular}

\section{Non-dispersive sensors}

Broadband, non-dispersive gas sensing is one of the simplest techniques to construct, and its great commercial significance is perhaps a result of this. Similar broadband measurements can be made in the mid infrared (in traditional non-dispersive infrared or NDIR), near infrared and UV / visible regions of the spectrum. Sensors can be built that are very compact, with NDIR sensors in recent times being built into a standard form factor consisting of a $16 \mathrm{~mm}$ long, $20 \mathrm{~mm}$ diameter cylinder. These sensors are low cost, having few components (a simple microbulb light source, gold coated reflective light path and integrated detector containing two or more filtered detection channels). For some gases, notably carbon dioxide, alternative (non-optical) technologies are unsuitable and therefore $\mathrm{CO}_{2}$ detection in low-cost, mass market applications often incorporates an NDIR sensor. These applications include heating, ventilation and air conditioning (HVAC) control, industrial safety especially in the brewing industry $\left(\mathrm{CO}_{2}\right.$ is an asphyxiant), and capnography (the measurement of time-resolved carbon dioxide concentration in exhaled breath) for patient monitoring for example during anaesthesia. 


\subsection{Principle of operation}

Typically, emission from a broadband source (such as a microbulb ${ }^{[79]}$ ) is passed through two filters, one covering the whole absorption band of the target gas (in the active channel), and the other covering a neighbouring non-absorbed region (the reference channel). Both filters must be carefully chosen to ensure that, ideally, no other gas species present are likely to be absorbed within their transmission windows. In modern NDIR designs, it is common for both detector channels, including filters, to be integrated into a single package, for example in a TO9 can $^{[80]}$. Further miniaturisation may also result from work to integrated detectors and filters on a single base substrate ${ }^{[81]}$. Typical filter characteristics for $\mathrm{CO}_{2}$ measurement are shown in Figure 14, alongside the gas absorption spectrum.

NDIR gas sensing relies on the strength of optical absorption in the mid IR, which can be of the order of 100 times greater than that in the near IR. Thus, even with short pathlengths $(3-10 \mathrm{~cm})$, using relatively unsophisticated sources (microbulbs) and uncooled detectors (pyroelectric or thermopile), respectable limits of detection may be achieved (eg 10-50 ppm for $\mathrm{CO}_{2}{ }^{[82]}$ ), and detection limits as low as $1 \mathrm{ppm}$ can be obtained using more sophisticated benchtop equipment ${ }^{[83]}$.

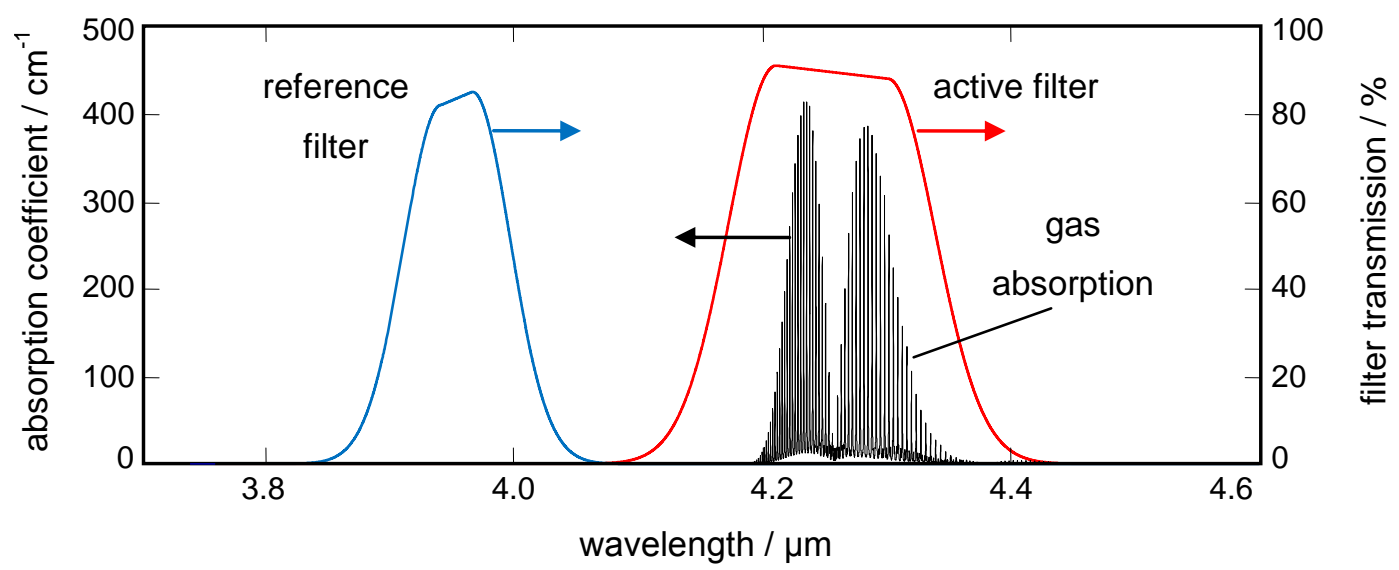

Figure 14. Illustration of NDIR measurement principle. The absorption spectrum of $\mathrm{CO}_{2}(100 \% \mathrm{vol}$, $1 \mathrm{~atm}$, calculated from Hitran $\left.{ }^{[20]}\right)$ is superimposed on the transmission spectra of active and reference channel filters (approximated from ref [80])

Transmission through the active channel comprises the integrated gas absorption:

$$
I=I_{0} \int E_{S}(\lambda) T_{A}(\lambda) \exp [-\alpha(\lambda) \ell] \mathrm{d} \lambda
$$

Where $E_{s}$ is the emission envelope of the source and $T_{A}$ is the transmission of the active channel filter. For low $\alpha \ell$, equation (5) is again linear with $\alpha$, and therefore with gas concentration.

The reference channel is used to compensate for changes in the emission of the source, which are assumed to affect the reference and active channel wavelengths in equal proportion. In the case of incandescent sources, these changes tend to result from either (i) temperature fluctuations of the filament, or (ii) gradual blackening of the inside of the glass envelope caused by filament evaporation; in either case the assumption is valid to first order. 
In the region of linear operation, we can adapt equation (2) to give;

$$
\alpha^{\prime} \ell=1-\frac{I_{A}}{I_{0 A}}
$$

Where the subscript $A$ denotes the active channel, and $\alpha^{\prime}$ is a pseudo absorption coefficient that follows from equation (5). Assuming that, in the absence of gas, the light transmitted through the reference channel is proportional to that transmitted through the active channel, we have;

$$
\alpha^{\prime} \ell=1-\frac{I_{0 R}}{I_{0 A}} \frac{I_{A}}{I_{R}}
$$

Where the subscript $\mathrm{R}$ denotes the reference channel. The value of $I_{0 \mathrm{R}} / I_{0 \mathrm{~A}}$ may be determined by flushing the sample cell with clean air. Figure 15 shows a schematic diagram of a simple NDIR gas sensor.

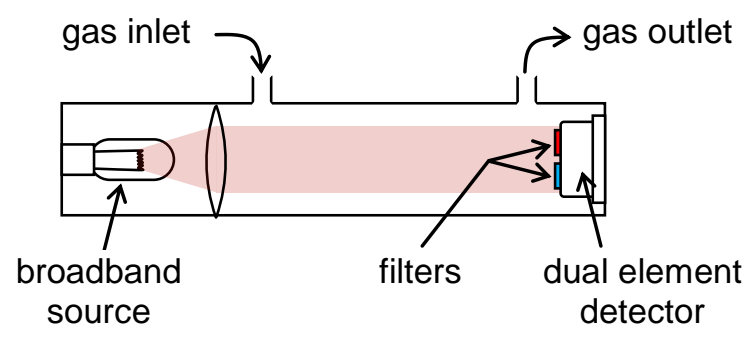

Figure 15. Schematic diagram of a typical non-dispersive gas sensor with optical pathlength in the range $3-20 \mathrm{~cm}$. The source and detector are usually placed inside the cell to avoid baseline drifts caused by variations in the background concentration, which are particularly important in the case of $\mathrm{CO}_{2}$ measurements.

Because broadband light sources are often highly divergent, detection limits over long pathlengths can be dependent on the source power emitted within a defined spectral window, and the detector noise. For the latter, a normalised figure of merit may be used, the specific detectivity or $D^{*}$, equal to the signal to noise ratio (SNR) when used in uniform irradiance, normalised for detector area $A_{d}$ and measurement bandwidth $\Delta f$.

$$
\mathrm{D} *=\frac{\sqrt{\mathrm{A}_{\mathrm{d}} \Delta f}}{\mathrm{NEP}}
$$

Where NEP is the noise equivalent power. The units of $D^{*}$ are typically expressed as $\mathrm{cm} \cdot \mathrm{Hz}^{1 / 2} \cdot \mathrm{W}^{-1}$.

The performance of NDIR systems can be estimated in terms of the minimum detectable change in optical power, $\Delta \mathrm{I} / \mathrm{I}$. For NDIR the figure corresponds to a noise equivalent absorbance where the "absorbance" concerned is an integrated effective absorbance over the measurement band. Aleksandrov et al have claimed an NEA better than $10^{-3}$ (100 ms response time $)^{[84]}$. In this case, a minimum detectable $\mathrm{CO}_{2}$ concentration of $25 \mathrm{ppm}$ was estimated, using a $4 \mathrm{~cm}$ pathlength.

Among many commercial implementations of this technology, the Li-Cor open path $\mathrm{CO}_{2} / \mathrm{H}_{2} \mathrm{O}$ analyser is notable for its good performance over long periods of unmanned operation ${ }^{[85]}$. The instrument achieves a reported limit of detection of $0.11 \mathrm{ppm} \mathrm{CO}_{2}$ with an optical pathlength of $12.5 \mathrm{~cm}$, operating in the $4.2 \mu \mathrm{m}$ measurement band. Its equivalent NEA was not reported, however comparison with the work of Aleksandrov et al above, in the same wavelength band, yields an estimate in the region of $10^{-5}$. For applications in eddy flux covariance measurement of greenhouse 
gas fluxes, accuracy is also a prime concern, and this has been reduced to $1 \%$ of the reading by careful consideration of a range of instrument factors.

\subsection{Light sources for NDIR}

Here we consider broadband sources used in non-dispersive measurements; lasers are considered later in section 6.3. The microbulbs used in conventional NDIR sensors have two main advantages; their spectral emission is relatively high (eg $2 \mathrm{~mW}$ per steradian in a FWHM bandwidth of $0.17 \mu \mathrm{m}$ at $\left.4.2 \mu \mathrm{m}^{[86]}\right)$ and the cost is low $\left(\$ 1-2^{[86]}\right)$.

However, as microbulbs are typically operated up to $3000 \mathrm{~K}$, their emission can contain a significant proportion of visible and short wave IR radiation which is not used in the measurement ${ }^{[87]}$. Puton et al have claimed that this additional radiation may cause spurious detection signals ${ }^{[88]}$, presumably if a small proportion of it passes unblocked through the filters and onto the detector. Microbulbs also have limited electronic modulation frequencies, up to $10 \mathrm{~Hz}$ being typical. Thermal detectors also tend to be optimised for use at low frequencies, with the result that $1-2 \mathrm{~Hz}$ operation is common, at which frequency $1 / f$ noise can be problematic. For certain applications, such as capnography, faster response times also dictate the use of frequencies of $30 \mathrm{~Hz}$ or more ${ }^{[89]}$. A final disadvantage of the standard microbulb is that its glass envelope has negligible transmission at wavelengths longer than $5 \mu \mathrm{m}^{[79]}$, and therefore for certain gases that absorb at longer wavelengths, alternative packaging is required. Emerging sources are often packaged in TO cans, enabling the use of flat windows that are available in a wide range of IR- transmitting materials.

Much recent research has therefore concentrated on development of sources that are both more spectrally efficient and capable of more rapid modulation frequencies. For improved modulation frequencies to have the greatest impact, thermal detectors also need to be optimised for higher frequency operation, otherwise semiconductor detectors (eg PbSe), which have bandwidths of $\mathrm{kHz}$ or more, must be used. The latter have improved $D^{*}$ values but are more costly and often require cooling.

The new sources can be categorised as follows;

- thin incandescent membranes, based on MEMs technology, some of which have engineered high emissivity surfaces,

- membranes with structured surfaces designed to improve their spectral efficiency at a given wavelength,

- light emitting diodes (LEDs).
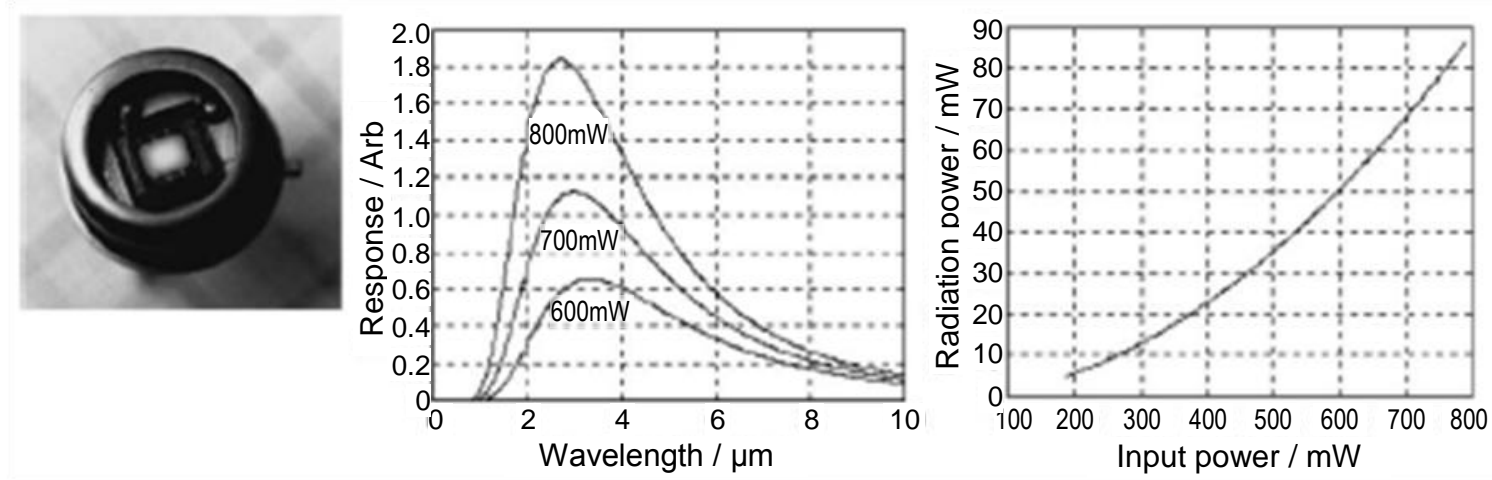

Figure 16. Emission parameters for a $1.7 \times 1.7 \mathrm{~mm}$ thermal emitter (Patinor 15-22). Taken from [90]. 
IR emitting membranes are essentially thin, flat equivalents of the coiled filaments used in conventional microbulbs. Their emission spectrum closely follows the Planck emission curve for a grey body (a blackbody whose emissivity is less than unity). Low temperature operation $\left(600-1400^{\circ} \mathrm{C}\right)$ gives improved spectral efficiency for the mid IR region and longer device lifetimes. MEMS manufacturing methods can be employed to develop thin films, either suspended in air or deposited as layers on a ceramic substrate, which can be modulated quickly because of their low thermal mass. Figure 16 shows typical emission parameters.

Modulation frequencies ( $3 \mathrm{~dB}$ points) of over $10 \mathrm{~Hz}$ have been achieved by several groups ${ }^{[01,92,93,94]}$. A maximum modulation frequency of $30 \mathrm{kHz}$ has even been proposed for a design with very small dimensions and therefore reduced thermal mass $(30 \mu \mathrm{m} \times 10 \mu \mathrm{m} \times 0.8 \mu \mathrm{m})^{[95]}$, however the compromise is a reduction in the total emitted power (in this case to $430 \mathrm{nW}$ ), which for a blackbody is directly proportional to the emitting area. One way of circumventing this compromise is to build an array of multiple emitters that are modulated synchronously. Cozzani et al have demonstrated $60 \mathrm{~mW}$ output in the range $9-12 \mu \mathrm{m}$ with a $4 \times 4$ array of $1.5 \times 1.5 \mathrm{~mm}$ emitters ${ }^{[96]}$, however their modulation frequency was not reported. An example of a single emitter is shown in Figure 17.

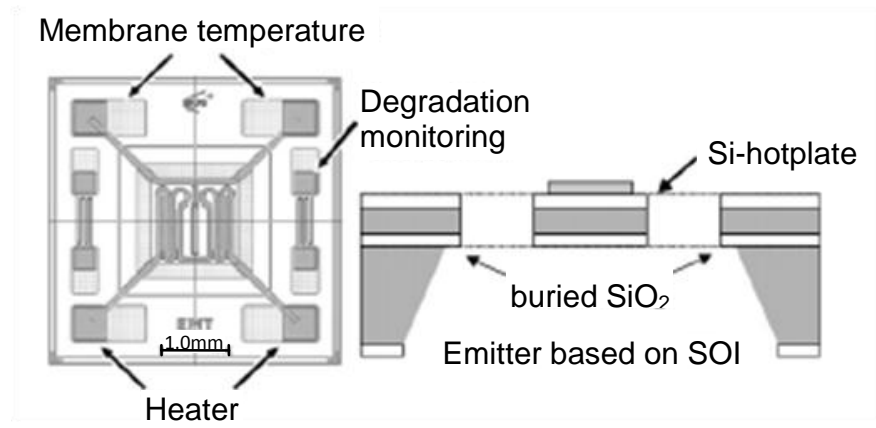

(a)

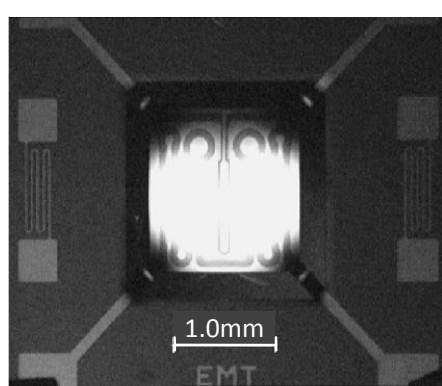

(b)

Figure 17. Example IR emitter fabricated from $\mathrm{SiO}_{2}-\mathrm{on}-\mathrm{Si}$ (silica on insulator, SOI) MEMS technology. (a) Mask layout, (b) visible emission recorded at $1000^{\circ} \mathrm{C}$, taken from [97].

Different coatings have been applied to confer improved properties, namely platinum black for increased emissivity compared to a plain metal surface ${ }^{[98]}, \mathrm{SnO}_{2}: \mathrm{Sb}$ for improved long-term stability at high operating temperatures (giving an estimated lifetime of 10 years at $950^{\circ} \mathrm{C}^{[97]}$ ) and $\mathrm{Si}_{3} \mathrm{~N}_{4}$ for longer wave $(9-12 \mu \mathrm{m})$, lower temperature $\left(600^{\circ} \mathrm{C}\right)$ operation ${ }^{[96]}$.

Microstructured coatings, with features on a scale similar to the target wavelength, have been used to improve spectral efficiency, though these have not yielded spectral emission above the level dictated by the Planck emission curve for unit emissivity. It has been shown that photonic crystal structures consisting of simple layer stacks or three dimensional structures ${ }^{[99,100]}$ can give a spectrally selective output, with low emission in the region of photonic bandgaps. Chan et al have proposed simple structures which yield distinct peaks at particular wavelengths ${ }^{[101]}$, in principle giving improved efficiency for detection of certain gases. A similar approach has also been experimentally demonstrated by Puscasu et a ${ }^{[102]}$ (as shown in Figure 18). Their devices have been used as both emitter and detector (in the manner of a bolometer) for gas detection ${ }^{[103]}$, giving a detection limit for $\mathrm{CO}_{2}$ of $1600 \mathrm{ppm}$ within a $24 \mathrm{~mm}$ pathlength. 


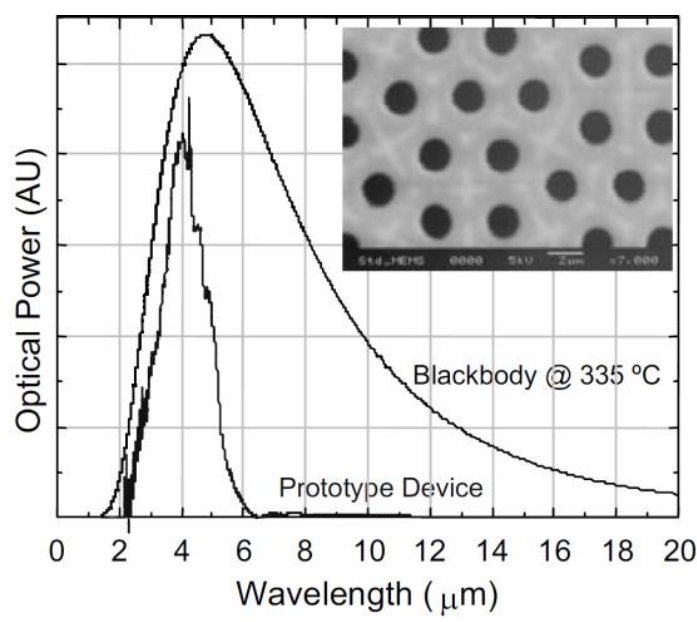

Figure 18. (a) Measured emission spectrum for a photonic crystal structure consisting of an array of holes with lattice spacing approximately $4.2 \mu \mathrm{m}$ (shown in inset), compared with emission from a blackbody of equivalent temperature and area. Taken from [104].

We can discuss the consequences of these developments for gas detection. Comparison of spectral emission in Figure 18 with the filter profiles in Figure 14 reveals a number of points. Firstly, device emission in the active channel has a sharper peak than the filtered emission from a microbulb, such that the emission is not quite flat across the gas absorption band. Therefore measurements may be somewhat sensitive to changes in lattice spacing caused by manufacturing tolerance or ambient temperature changes, or to changes in the envelope of the absorption band of the target gas, which may also be caused by temperature changes. This may be mitigated in time by improved modelling and fabrication techniques aimed at optimising devices for gas detection. Secondly, this technology yields improvements in spectral power efficiency rather than in absolute power levels, therefore its impact will be greatest in portable applications where power use is of greatest concern. Finally, as we find for tunable diode lasers (see section 6) the ultimate availability of commercial devices will likely depend to a great extent on the size of the market for target gases at particular wavelengths; the more specific the emission wavelength, the more specific the market sectors.

Indeed, this situation is the case for recently developed mid IR LEDs, which are designed to emit at wavelengths targeting particular gases. They offer superior spectral efficiency than thermal sources, with emission better matched to the gas absorption band ${ }^{[105]}$. Emission can be temperaturedependent, as Figure 19 shows. These devices are capable of much higher modulation rates than thermal sources, but have only been available at relatively low spectral power levels in comparison ${ }^{[83]}$. Both attributes lend these devices to be used with semiconductor, rather than thermal, detectors, which have better detectivity and bandwidths of $\mathrm{kHz}$ or more. In fact, the same LED materials also allow use as uncooled semiconductor sensors with matched spectral characteristics; if used with an LED of the same construction, the two are referred to as an optopair. Aleksandrov et al have analysed SNRs for gas detection with various LED / detector combinations, with a limit of detection for methane at $3.3 \mu \mathrm{m}$ or $170 \mathrm{ppm}{ }^{[105]}$, and in later work similarly a limit for $\mathrm{CO}_{2}$ of $25 \mathrm{ppm}^{[84]}$. 


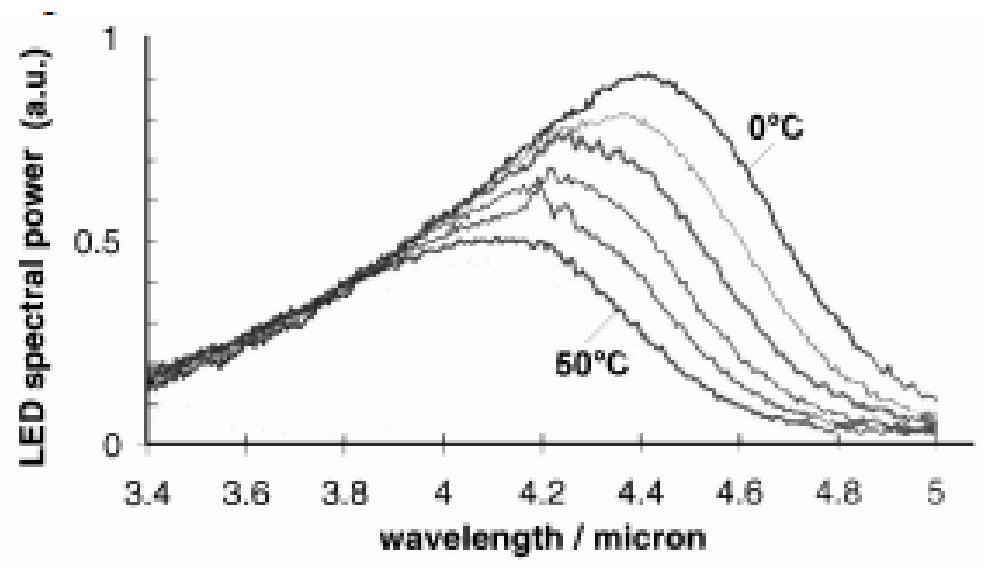

Figure 19. Typical temperature dependence of LED spectral emission, taken from [86]. The peak wavelength is strongly wavelength - dependent, and emitted power falls at higher temperatures.

LEDs emit greater peak power levels when operated in a pulsed mode, however for gas detection the best SNRs are found with a 50/50 duty cycle square wave modulation ${ }^{[103]}$, thus the CW power is of greater interest when the best detection limits are required. However, for applications that require very low power, such as certain portable sensors, pulsed operation may be used to give higher efficiencies. For example, Danilova et al have observed peak emission of $7 \mathrm{~mW}$ with a $50 \%$ duty cycle and $300 \mathrm{~mA}$ current, rising to $190 \mathrm{~mW}$ with a $0.5 \%$ duty cycle and $1.4 \mathrm{~A}$ current, for $1.9-2.2 \mu \mathrm{m}$ LEDs operated at $300 \mathrm{~K}^{[106]}$. Here, a $50 \%$ duty cycle has $3.7 \mathrm{x}$ higher RMS emitted power, whereas a $0.5 \%$ duty cycle has $5.8 x$ better power efficiency.

Table 5. Sample of reported performance of room temperature mid IR semiconductor sources.

\begin{tabular}{|c|c|c|c|c|c|c|c|}
\hline Mechanism $^{a}$ & Active region & $\frac{\frac{\text { Peak }}{\text { wavelength }}}{\underline{/ \mu \mathrm{m}}}$ & $\frac{\text { Power }}{\text { / mW }}$ & $\begin{array}{l}\frac{\text { Drive }}{\text { current }} \\
\text { / mA }\end{array}$ & $\begin{array}{l}\text { Duty } \\
\underline{\text { ratio }}\end{array}$ & $\frac{\text { Area }}{1 \mathrm{~mm}^{2}}$ & $\underline{\text { Reference }}$ \\
\hline NL LED & InAs & - & 1.1 & 200 & $50 \%$ & 0.79 & [107] \\
\hline NL LED & $\ln A s / \ln A s S b$ & 4.3 & 0.03 & 200 & $\mathrm{CW}$ & 0.04 & [108] \\
\hline NL LED & $\ln A s / \ln A s S b$ & 4.8 & 0.01 & 100 & $50 \%$ & 0.02 & [109] \\
\hline NL LED & $\begin{array}{l}\text { GaSb / GalnAsSb } \\
\text { / AlGaAsSb }\end{array}$ & 1.95 & 2.5 & 400 & $\mathrm{CW}$ & 0.09 & [110] \\
\hline OP & $\mathrm{PbSe}$ & $4-5$ & 2 & 5000 & $\mathrm{CW}$ & 0.2 & [111] \\
\hline QC LED & $\begin{array}{l}\text { InAs / GalnSb / } \\
\text { AISb }\end{array}$ & 3.8 & 0.22 & 15 & $\mathrm{CW}$ & 0.01 & [112] \\
\hline NL LED & InAISb & 5.3 & 0.27 & 100 & $\mathrm{CW}$ & 1 & [113] \\
\hline NL LED & InAISb & 4.27 & 0.3 & 250 & $50 \%$ & 0.07 & [114] \\
\hline EL LED & InAs & 3.3 & 0.17 & 200 & $50 \%$ & 0.2 & [115] \\
\hline $\begin{array}{l}\text { NL n€ } \\
\text { electr }\end{array}$ & \multicolumn{7}{|c|}{$\begin{array}{l}\text { NL negative luminescence, OP optically pumped, QC quantum cascade (see section 6.3), EL } \\
\text { electroluminescence }\end{array}$} \\
\hline
\end{tabular}


In 2002, Smith et al completed a survey of LED characteristics for the period 1996-2001 ${ }^{[86]}$. Much of the cited work exploited a phenomenon known as negative luminescence $(\mathrm{NL})^{[116]}$. Since then, alternative light emission mechanisms have been observed including electroluminescence (EL) and emission from quantum cascade LEDs (QC LED). Semiconductor devices based on optical pumping (OP) have also been described; although not strictly LEDs, we include them here. From a gas detection perspective the emission mechanism is a means to an end, enabling higher powers and greater wavelength coverage. Following on from the review of Smith et al ${ }^{[83]}$, a sample of reported recent performance is compared in Table 5 , but this is by no means exhaustive.

Because of the relatively low level of light emitted from these sources, various attempts have been made to improve optical coupling efficiencies. Techniques applied to emitters have frequently also been applied to detectors in gas detection systems, and are summarised in Table 6.

Table 6. Summary of techniques used to improve output coupling from mid IR LEDs.

\begin{tabular}{|l|c|c|c|}
\hline \multirow{2}{*}{ Technique } & \multicolumn{2}{|c|}{ Improvement factor } & \multirow{2}{*}{ Reference } \\
\cline { 2 - 3 } & $\underline{\text { experimental }}$ & predicted & \\
\hline High NA immersion lens & 5 & 16 & {$[117]$} \\
\hline Inclined mesa sidewalls and surface roughening & 2 & - & {$[118]$} \\
\hline Resonant cavities & 2 & 22 & {$[119]$} \\
\hline Surface grating structures plus rear mirror & - & 22 & {$[120]$} \\
\hline 2D photonic crystal surface structures & Up to 6 & - & {$[121]$} \\
\hline
\end{tabular}

UV LEDs are now commercially available ${ }^{[122]}$ with centre wavelengths from $240 \pm 5 \mathrm{~nm}$ and typical emission $\geq 0.3 \mathrm{~mW}$. Under research are LEDs with shorter wavelength emission; Figure 20 illustrates the latest results, including AIGaN based LEDs with centre wavelengths down to $222 \mathrm{~nm}^{[123]}$, and AIN based LEDs down to $211 \mathrm{~nm}^{[124]}$, almost providing sufficient coverage for the entire $200-400 \mathrm{~nm}$ region.

The advantages are similar to those provided by mid IR LEDs, with light being easier to control, having a smaller emission area, enabling better use of the overall output. Although power levels are low, spectral power (power available in the emission bandwidth) is very high, and power efficiency is relatively high. Ultimately, lifetimes should be longer than for other lamp technologies such as deuterium or xenon flashlamps. Finally, source flicker noise, which can be the limiting noise in high quality spectrophotometry ${ }^{[18]}$, tends to be lower for LEDs than for incandescent or discharge sources $^{[125]}$, and sources may be more easily modulated to give better noise rejection. An issue that remains is that the centre wavelength of LED emission is generally temperature-sensitive, such that temperature control or compensation may be required. 


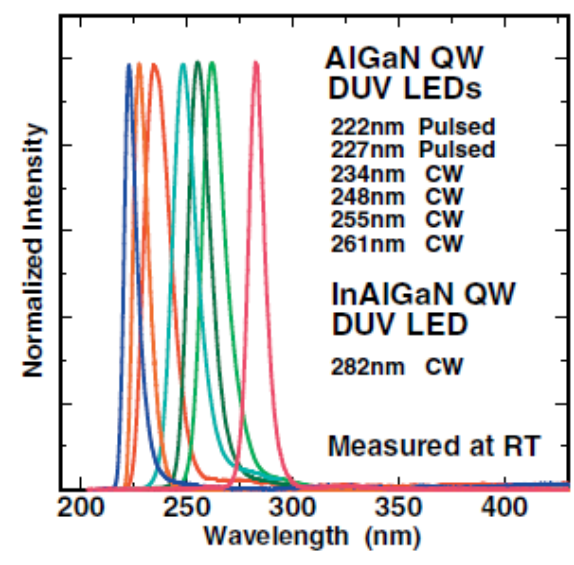

(a)

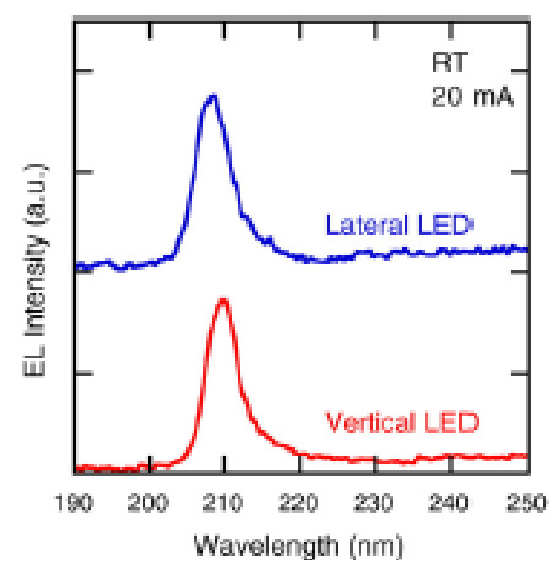

(b)

Figure 20. Recent spectral emission results of UV LED research, covering centre wavelengths from $211 \mathrm{~nm}$ to $282 \mathrm{~nm}$ : (a) AIGaN based LEDs, taken from [123], (b) an AIN based LED, taken from [124].

\subsection{Non-dispersive gas cell development}

Over the last decade, the commercial market has become populated with small footprint gas sensors based on the NDIR principle ${ }^{[126,127]}$. The dimensions of these sensors (a cylinder $20 \mathrm{~mm}$ diameter $x 16$ $\mathrm{mm}$ high) follow a gas industry standard format. Thus, equipment manufacturers need not alter the dimensions of their products when switching to NDIR sensors, and in some cases even the first stage electronics design is also similar. However, the small footprint is limiting and other configurations are also of interest, especially where they provide an increased pathlength.

Research in this area has concentrated on the following requirements: (i) the need for compact cells, (ii) the need to maximise the proportion of light coupled through the cell to the detector, in order to overcome the detector noise limit, and (iii) in applications requiring very low limits of detection, the need for very long pathlength cells.

A key to the miniaturisation of this technology has been the integration of multiple detectors and filters into a small single package, typically a $9 \mathrm{~mm}$ diameter TO can. Fonollosa et al have extended this to include an high NA Fresnel lens formed in silicon (transmissive in the mid IR) to make a light-efficient and ultra-compact dual band detector, as shown in Figure $21^{[128]}$. The authors also proposed use of an antireflection coating, to lessen the effects of silicon's relatively high refractive index. The detector module has been integrated into a system for measuring ethylene in agricultural storage, with a 30 ppm detection limit ${ }^{[129]}$. 


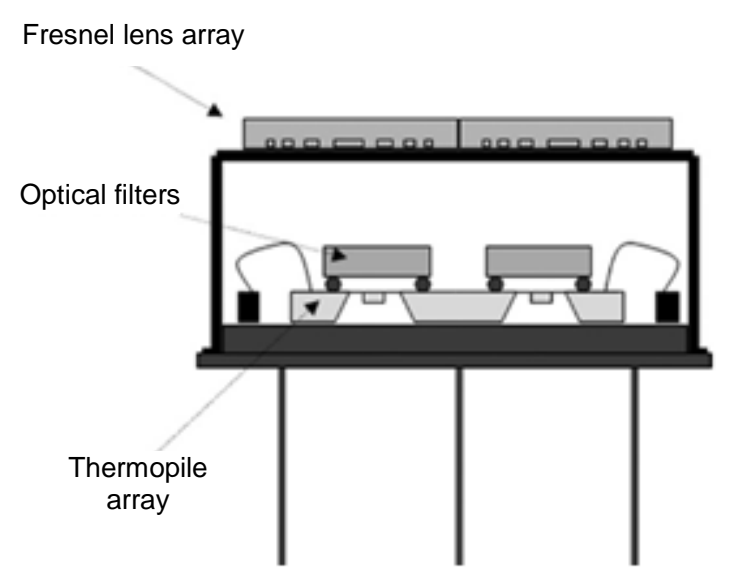

(a)

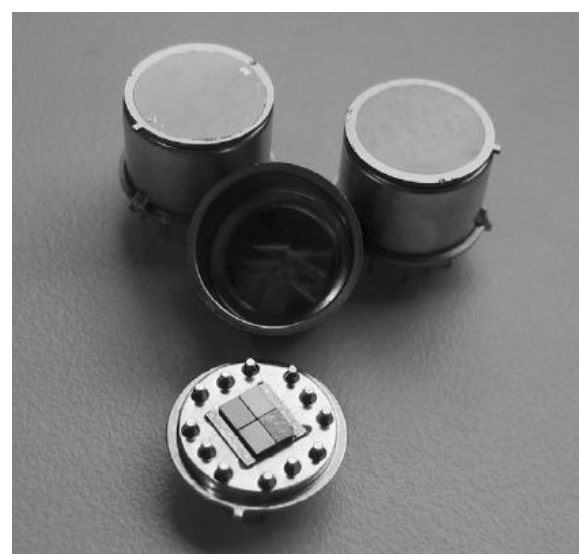

(b)

Figure 21. Integrated filters, detectors and Fresnel lenses in a TO8 can. (a) Cross-section, (b) constructed device in a 9mm diameter TO8 can. Taken from Fonollosa et al, 2008 ${ }^{[128]}$.

Non-dispersive gas sensing has been brought a step closer to dispersive spectrophotometric analysis by the development of IR filter arrays. Fonseca et al have demonstrated a limited $3 \times 3$ array of thermopiles each with its own specific narrowband filter, all packaged in a $6 \mathrm{~mm}$ device as shown in Figure $22^{[130]}$. It is claimed that the following gases may be discriminated: (i) ethanol, acetone and isopropanol, (ii) $\mathrm{CO}_{2}$ and methane. Linear filter arrays are also now available from commercial manufacturers ${ }^{[131]}$.

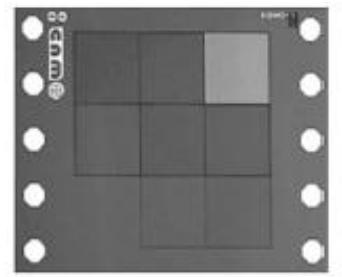

(a)

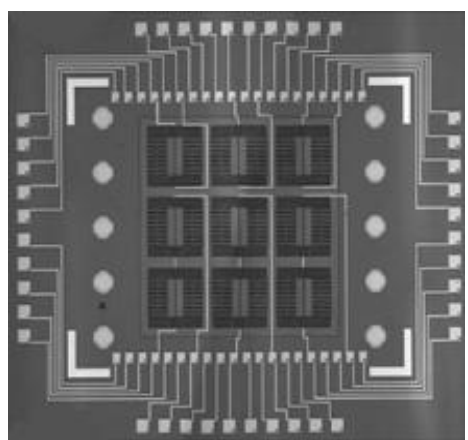

(b)

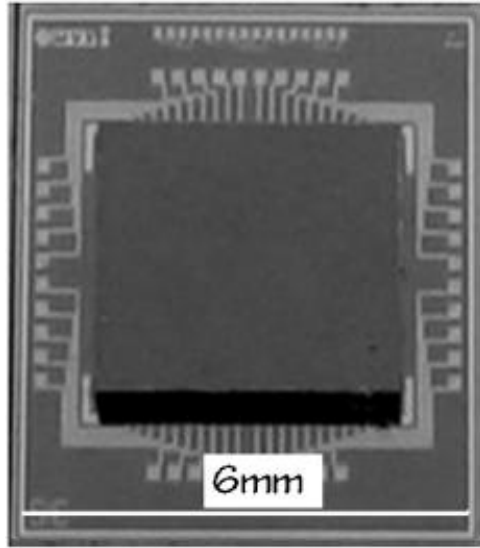

(c)

Figure 22. Filtered thermopile array for multispecies NDIR, taken from [130]. (a) Interference filters, (b) thermopile array, (c) assembled device.

At the other extreme of gas "cell" development, NDIR spectroscopy has formed the basis of remote, open path gas leak imaging in industrial applications using thermal imaging arrays operating in mid IR regions that overlap the absorption spectra of gases of interest. So-called passive gas imaging relies on the use of a background source of radiation, and a filtered detector provides gas selectivity. Reference detectors are typically not used; the presence of a gas leak is either inferred by eye from an image ${ }^{[132]}$, or by subtraction of the background scene in the absence of gas ${ }^{[133]}$. As long as there is a 
temperature difference between the background scene and the target gas, contrast will result in the image. Figure 23 shows a false colour image of a low pressure gas leak taken under ideal conditions (with a heated background) using background image subtraction. However, the main disadvantage of this technique is that it does not fail safe unless sufficient temperature contrast can be guaranteed; then, the lack of gas in an image does not guarantee the lack of a leak.

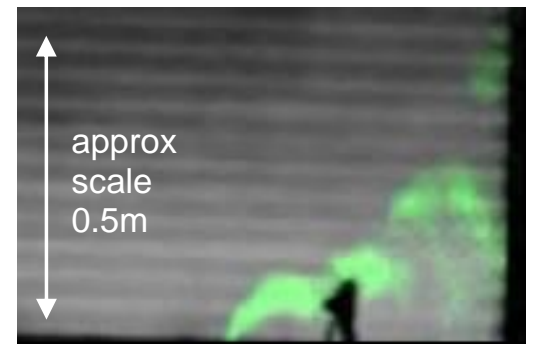

(a)
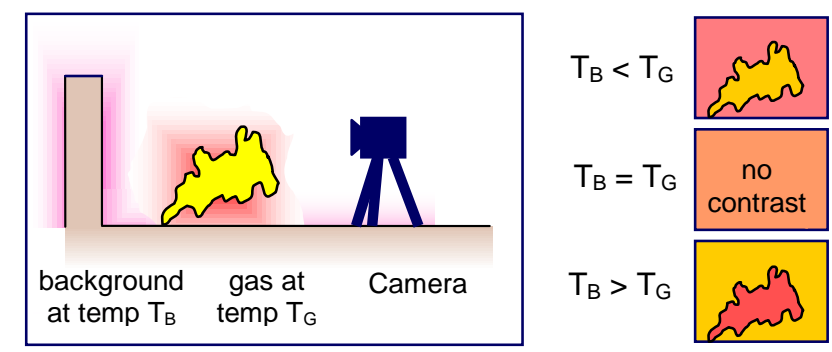

(b)

Figure 23. (a) False colour image of an outdoor methane leak of 5 litre $\min ^{-1}$ with a temperature contrast $\Delta T$ of approximately $30 \mathrm{~K}$, modified from [133]. (b) Representation of measurement principle.

\subsection{Correlation spectroscopy}

Correlation spectroscopy can be considered a development of NDIR, in which the broadband filter is replaced by or augmented with a reference gas cell containing a known quantity of the target gas. With a sufficiently large absorption coefficient in the cell, only light with wavelengths that are not absorbed by the target gas can reach the detector. The reference detector output is compared with that from an equivalent channel containing no reference gas; by analogy with NDIR we will call this the active channel. By normalising the two intensities and subtracting them, it is therefore possible to calculate the level of light absorption at wavelengths corresponding only to those absorbed by the target gas. Figure 24 shows a simple example of relevant spectra at different stages in the system.

Correlation spectroscopy can therefore provide selectivity to the target gas using relatively simple equipment, using the gas itself to provide a high resolution matched filter. Gas selectivity is exemplified by Lou et al who developed a system using a low cost multimode diode laser to detect $\mathrm{CO}_{2}$ and $\mathrm{CO}$ specifically in mixtures of the two, obtaining accuracies of $2 \%$ and $1 \%$ respectively ${ }^{[134]}$. 

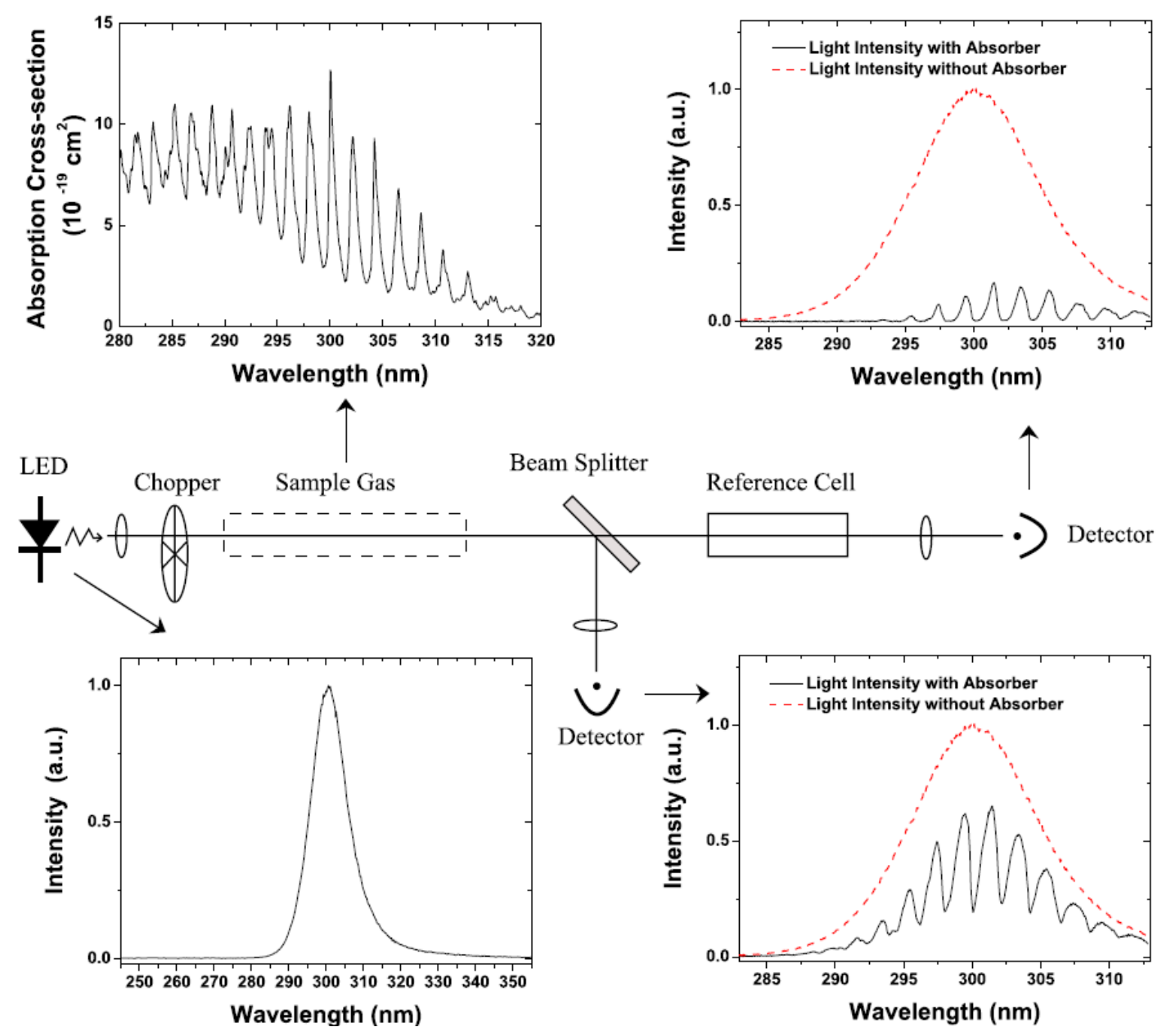

Figure 24. Example of correlation spectroscopy using a UV LED as a source, illustrating spectra for source emission, target gas absorption, and received light at the two detectors. Taken from [135].

The methods by which the reference beam (in the presence of gas in the reference cell) and the active beam are differentiated accounts for the main design differences between correlation systems. Ideally, the two beams will be well matched in all respects other than the absorption (or not) of the reference gas. However, in practice some separation of the beams becomes inevitable such that researchers need to correct for minor differences in reflectivity of cell windows or interference fringes generated by low finesse etalons in the system ${ }^{[23]}$. Such differences can be responsible for the limiting signal to noise ratio and drift effects in these systems. It was for this reason that Dakin et al developed a number of techniques with which to modulate the actual gas absorption within the reference cell itself, via pressure modulation or the Stark effect ${ }^{[136]}$, giving otherwise well matched reference and active beams, separated in time (at different phases of the modulation cycle) rather than space (see Figure 25).

A similar correlation principle can be employed using a Fabry-Perot interferometer (FPI) to provide an artificial gas reference with equally spaced transmission lines whose spectral position can be modulated. The technique can only be used to detect gases with evenly spaced absorption lines whose spacing corresponds to the free spectral range (FSR) of an FPI of practicable dimensions; fortunately this includes the IR spectra of many simple compounds whose spectra exhibit rotational fine structure. Vargas-Rodríguez and Rutt have built optimised systems on this basis for $\mathrm{CH}_{4}, \mathrm{CO}$ and $\mathrm{CO}_{2}$, each with a required FPI cavity length of between 0.5 and $3 \mathrm{~mm}^{[137]}$. A broadband thermal emitter 
was used as the source and their system had an optical pathlength of $1.4 \mathrm{~cm}$. Detector noise - limited minimum detectable concentrations were estimated to be $5 \mathrm{ppm}, 170 \mathrm{ppm}$ and 100ppm respectively for a $10 \mathrm{~s}$ response time.

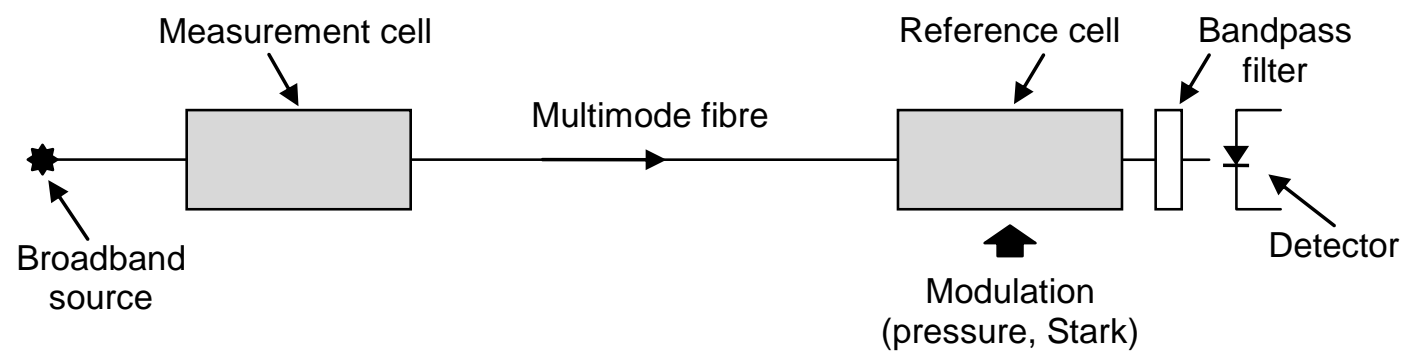

Figure 25. Modulation of the absorption within the gas reference cell allows a correlation system to have matched reference and active beams. After [136].

If this degree of beam matching cannot be achieved, the next best option may be to match the active and reference beams while in the sample cell itself, this being most likely to degrade over time in an instrument (because of exposure to samples that can be dusty or aggressive). Dakin et al have developed systems based on so-called complementary source modulation ${ }^{[138]}$, with an estimated limit of detection of $\Delta \mathrm{I} / \mathrm{I}$ of $2 \times 10^{-6}$. As Figure 26 shows, light from the two sources is modulated in antiphase, with the output from only one of the sources passing though the reference cell. Cheung et al extended this principle to use a common source, used in reference and active channels simultaneously, with a delay path in one causing a phase difference to appear when they were recombined $^{[139]}$. They estimated a minimum detectable acetylene concentration of $6 \mathrm{ppm}$ over a pathlength of $6 \mathrm{~cm}$, corresponding to a minimum detectable change in light level $\Delta \mathrm{I} / \mathrm{I}$ of $5 \times 10^{-7}$, however this was not explicitly demonstrated. Corman et al have also developed a micro miniature correlation system in which the beam separation was less than $2 \mathrm{~mm}$ and the total path traversed by the light was of the order of $10-20 \mathrm{~mm}^{[140]}$. This is likely to reduce the scale of thermal mismatch as well as providing a compact and low power system, however the limiting performance of the device was not explored. Finally, Austin et al have developed a correlation system around a reference cell made from photonic bandgap fibre with a hollow core of diameter $14.4 \mu \mathrm{m}^{[141]}$. The advantages of this system were a reduced reliance on bulk optics and a very small reference cell volume $(5 \mu \mathrm{l})$.

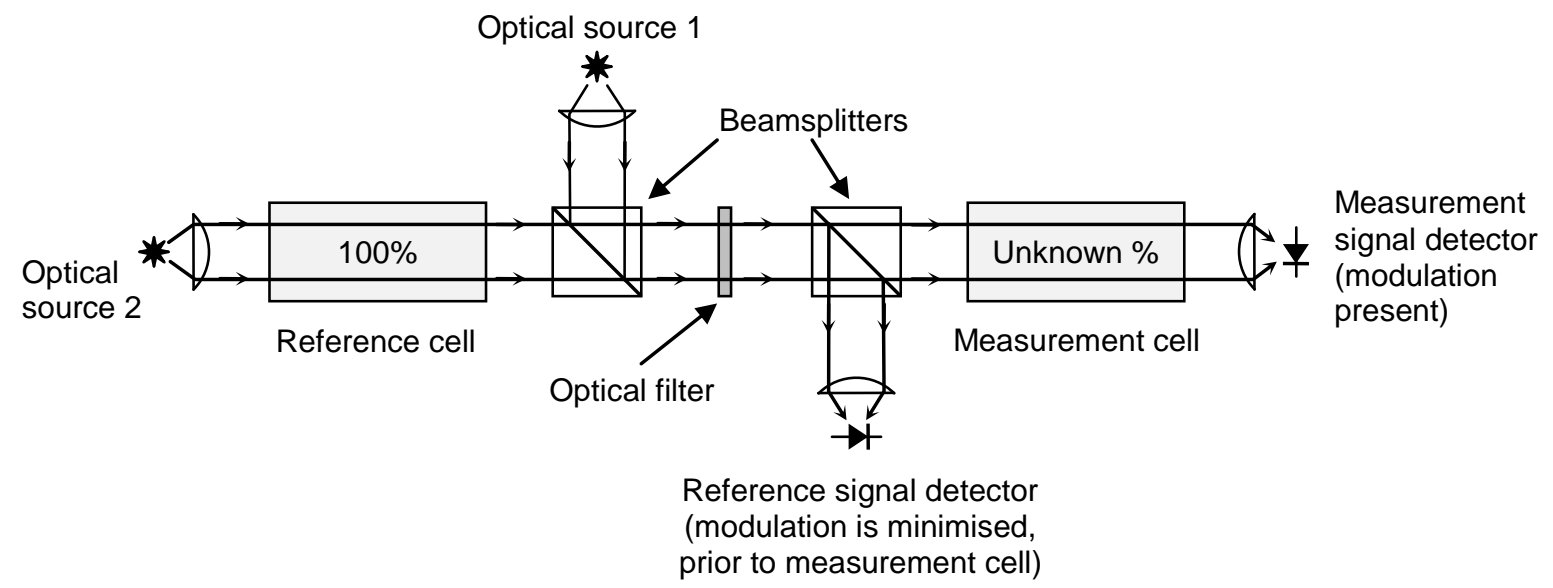

Figure 26. A gas correlation system based on the complementary source modulation technique. After Dakin et al [138]. 
Problems with the correlation approach can occur with linearity and calibration ${ }^{[137]}$, and with gas selectivity, should a proportion of the target gas lines overlap with those of other species ${ }^{[23]}$. Changes in atmospheric temperature and pressure in either the sample or reference cells can affect the linewidth of narrow gas absorption lines and therefore introduce calibration errors ${ }^{[23]}$. A further problem is the need to maintain a reference cell with a fixed concentration of the target gas that is stable over the lifetime or calibration interval of the instrument, ruling out the use of reactive or unstable gases. Indeed, Kebabian et al have found that, in the case of $\mathrm{NO}_{2}$ detection, a temperature-dependent equilibrium between the target gas and its dimer in the reference cell caused thermal drifts in the output at the level of $0.5 \mathrm{ppm} \mathrm{K}^{-1}$, responsible for the limiting performance of their instrument ${ }^{[142]}$.

Finally, correlation spectroscopy has found application in gas imaging through the work of Sandsten et $a{ }^{[143]}$. An image (for example of a petrochemical plant) is split through two arms of a gas correlation telescope, one of which contains a reference gas cell. The two images are then captured on precisely aligned CCD cameras and subtracted to form an image of the path-integrated concentration of the target gas species, which can be overlaid as a false colour image on a separate black and white image of the background scene, as shown in Figure 27. Use of the reference cell allows real-time subtraction of the background, allowing false colour imaging without the need to acquire a stable, gasfree background image.

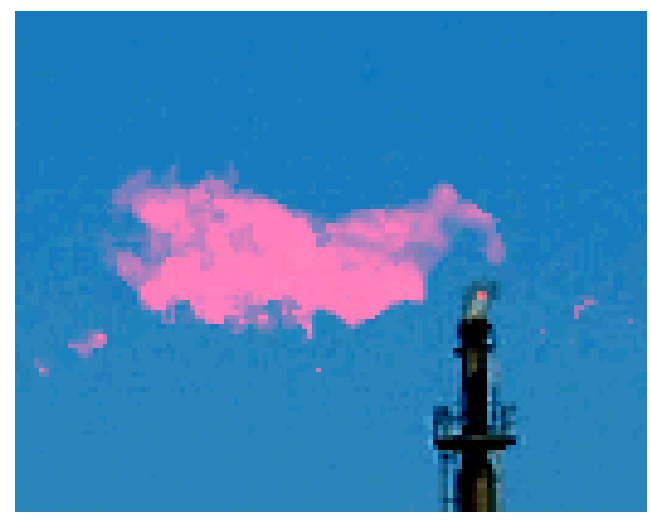

Figure 27. False colour image of ethane leaking from a polyethylene plant, taken using a gas correlation imaging camera using background radiation as the light source. Taken from [143].

\section{Spectrophotometry}

In contrast to the non-dispersive techniques described above, spectrophotometry is dispersive in the sense that the spectrum from a broadband source is dispersed by a wavelength - selective element such as a grating. Multiple gases may be detected provided that their absorption lines fall within the wavelength range of the spectrometer, and regions can often be found with little or no absorption to act as a reference, in the manner of the reference measurement in NDIR. The ability to screen for the presence of a large number of unknown compounds, and to identify and measure their concentrations, can be important, for example for first responders in security applications ${ }^{[144]}$.

In the UV-visible region of the spectrum are a number of important gases with strong absorptions such as the BTEX compounds (benzene, toluene, ethlybenzene and xylene), $\mathrm{NO}_{\mathrm{x}}$ compounds, ozone, hydrogen sulfide $\left(\mathrm{H}_{2} \mathrm{~S}\right)$ and sulphur dioxide $\left(\mathrm{SO}_{2}\right)$ amongst others. Absorption strengths in the mid IR 
are also sufficiently strong for good quality spectrophotometric measurements of gases to be made. However, use of the near IR spectrum is limited by the relatively weak absorption strengths of gases coupled with the limited signal to noise ratios of spectrophotometers compared with alternative methods (non-dispersive and tunable diode laser), the latter using discrete detectors and analogue first stage amplification.

UV absorption spectroscopy is the basis of a number of commercial techniques to analyse gases, one example having a claimed sub ppm limit of detection for $\mathrm{H}_{2} \mathrm{~S}$, mercaptans and $\mathrm{SO}_{2}$ in process streams ${ }^{[145]}$. Typical broadband light sources include deuterium or xenon lamps in the UV and incandescent lamps in the near IR. These may be fibre optically coupled so that the electronics can be housed separately from the gas cell.

However, UV spectral measurements of different gas species frequently overlap; to discriminate individual gases, spectra must be deconvolved, often using a partial least squares method. Because individual gas lines are often not fully resolved, the precise alignment of those lines with the spectral bins used to sample them can affect the measurements to a significant degree (up to $70 \%$ error ${ }^{[146]}$ ). For this reason, developers often calibrate their instruments using spectra measured with the instrument itself, rather than with library spectra. The nature and variability of the spectral background is also an important consideration when using such techniques, and in some applications may be the most important performance-limiting factor.

Perhaps the most straightforward configuration for compact spectrophotometry is a simple single pass gas cell, fibre optic coupled at one end to a light source and at the other to a diode array spectrophotometer, as shown in Figure 28. For example, this configuration has been used by Xu et al to detect $1 \mathrm{ppm} \mathrm{SO} \mathrm{SO}_{2}$ (with a deuterium lamp and $35 \mathrm{~cm}$ pathlength) ${ }^{[147]}$ and by Degner et al to make sub ppm measurements of ozone, $\mathrm{NO}_{2}$ and $\mathrm{SO}_{2}$ (LED source and $4 \mathrm{~cm}$ pathlength) ${ }^{[148]}$.

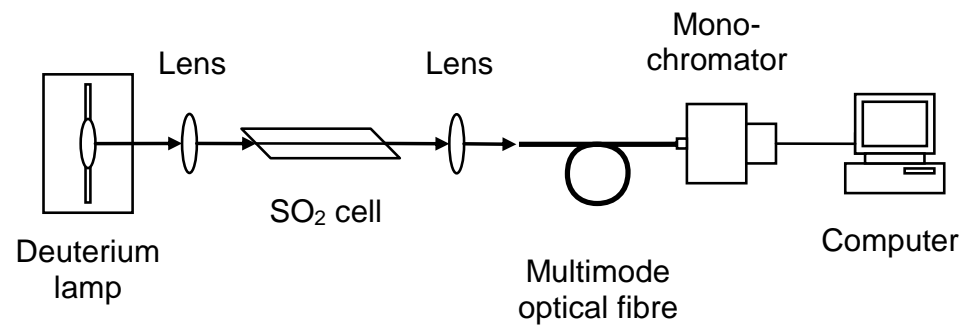

Figure 28. Simple configuration for spectrophotometric gas sensing using a fixed pathlength gas cell. After [147].

A second configuration in common use is used to make open path measurements over distance of tens to hundreds of metres, for environmental applications such as urban air quality monitoring or measuring volcano emissions. This is often (though not exclusively) combined with a spectral processing technique known as DOAS (differential optical absorption spectroscopy) in which, as the name suggests, measured spectra are pre-processed to give a first differential of the absorption as a function of wavelength ${ }^{[149]}$. Subsequent processing to extract overlapping absorption bands is eased by the reduced spectral baseline and increased sensitivity to sharper peaks. Absorbances as low as $2 \times 10^{-5}$ to $2 \times 10^{-4}$ can be detected ${ }^{[150]}$. More advanced algorithms have also been developed to enable low concentration measurements to be made over shorter pathlengths typical of industrial applications $^{[151]}$. 


\subsection{Spectrophotometer elements}

New designs for compact spectrophotometers have resulted from both advances in manufacturing techniques and from ingenuity on the part of developers. The field has been comprehensively reviewed by Wolffenbuttel in 2004 ${ }^{[152]}$, Crocombe in 2008 ${ }^{[153]}$ and Schuler et al in 2009 ${ }^{[154]}$ (MEMs devices). The aims are to create systems that are simple, compact and have low power consumption, for routine use in permanently installed or portable monitors, with the added option of optical fibre coupling such that the light source and spectrometer may be located remotely from the sample. A sample of recent developments is summarised in Table 7. Some have been developed primarily for near IR spectroscopy of liquid samples in the process industries and are therefore of indirect interest to gas detection, but have been included for completeness.

There has been great progress in the development of small ( $\mathrm{a}$ few $\mathrm{cm}$ long) diode array spectrometers capable of measuring spectra in the UV-visible and near IR with moderate to good spectral resolution $\left(0.04 \mathrm{~nm}\right.$ in the $\left.U V^{[155]}\right)$, sufficient to identify individual gas species. Figure 29 shows a schematic diagram. This has been combined with developments in $\mathrm{Si}$ or InGaAs diode arrays using CCD or CMOS technology. However, InGaAs arrays are relatively costly, bringing instrument prices to around $\$ 20,000^{[156]}$, therefore in this region alternative solutions have been sought that use discrete detectors, often using developments from the telecommunications industry (for example in TDLS - see section $6)$.

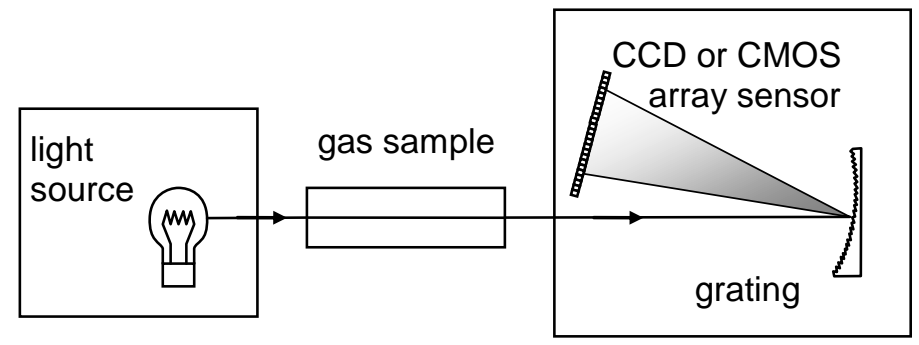

Figure 29. Schematic diagram of a CCD or CMOS array spectrometer.

A number of groups have developed MEMs-actuated Fabry-Perot filters for use in spectroscopy ${ }^{[154]}$. For example, Neumann et al have developed and commercialised a tunable Fabry-Perot filter to replace the discrete filters typical of NDIR detector modules ${ }^{[157]}$. Their design employs a MEMS based etalon with an effective finesse of 46 , giving a filter bandwidth of $86 \mathrm{~nm}$ at a centre wavelength of $4 \mu \mathrm{m}$ $(\Delta \lambda / \lambda \sim 2 \%)$. The effective tuning range that can be achieved using a single device could be used to measure several gases with neighbouring absorption bands, for example Figure 30 shows tuning over the range $3.9-5 \mu \mathrm{m}$. The team has also extended the wavelength capability to cover the 8-11 $\mu \mathrm{m}$ range ${ }^{[158]}$.

Vaisala have integrated a similar tunable Fabry-Perot filter into their Carbocap ${ }^{\mathrm{TM}}$ carbon dioxide monitor, capable of detecting $30 \mathrm{ppm} \mathrm{CO}_{2}$ at $4.2 \mu \mathrm{m}^{[159]}$. The principle has been taken a stage further by the fabrication of a full miniature optical bench on a $14 \mathrm{~mm}$ base ${ }^{[156]}$ (see Figure 31), which has been commercially developed by Axsun Technologies Inc. 


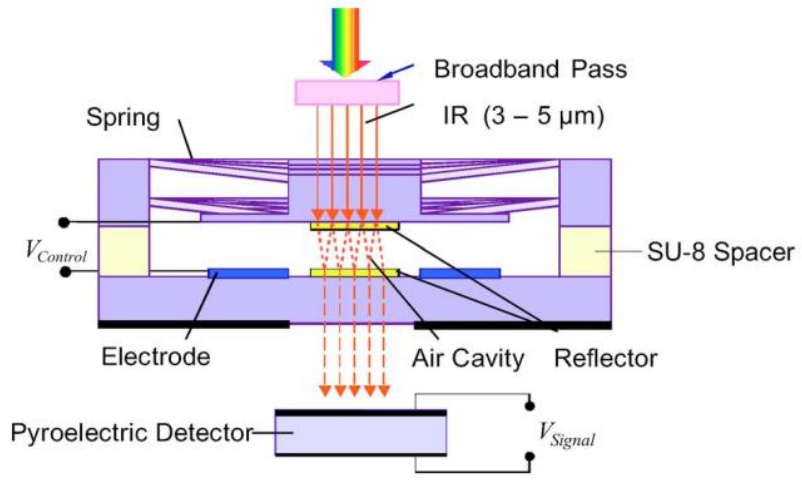

(a)

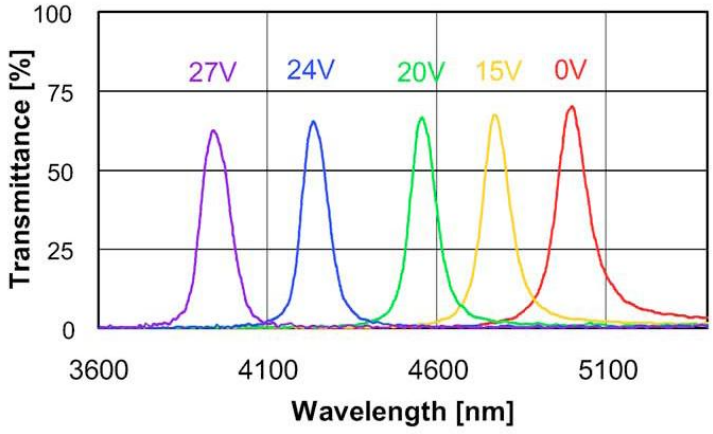

(b)

Figure 30. Tunable MEMS based Fabry-Perot filter for use in NDIR based gas detection. (a) Device design, (b) transmission spectra obtained at different drive voltages. Taken from [157].

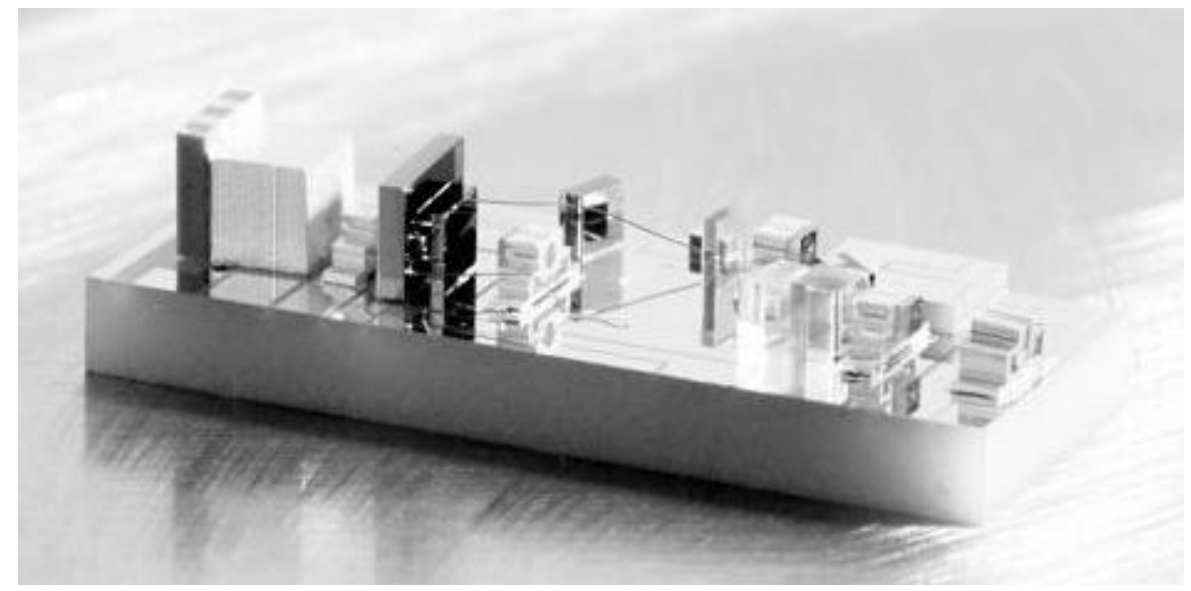

Figure 31. Micro-optical bench (14 mm long) incorporating free space micro optics, a tunable FabryPerot filter and fibre optic coupling. Taken from [156].

Another theme has been the development of compact interferometric spectrometers, including Fourier Transform (FT) spectrometers (FTIR or FTUV), which measure an interferogram whose transform yields the required transmission spectrum. FT spectrometers have two well-known advantages, both of which contribute to an improved signal to noise ratio compared to a spectrometer using a moveable grating with a discrete detector. These are higher optical throughput (Jacquinot's advantage) and the so-called multiplex advantage, whereby a spectrum containing $n$ spectral bins requires a measurement time $t \propto n$ for the moveable grating spectrometer compared with $t \propto n^{1 / 2}$ for a Fourier Transform spectrometer ${ }^{[18]}$.

High quality laboratory FT spectrometers are typically based on a Michelson interferometer, one arm of which employs a moving mirror, the spectral resolution being inversely related to the optical path difference resulting from mirror displacement. Therefore high resolution FT spectroscopy necessarily requires a large footprint instrument. These spectrometers can also be complex and difficult to maintain, so the development of compact interferometric spectrometers containing no moving parts, or MEMS-actuated parts, has been an important achievement. Nevertheless, a reduction in size 
necessarily results in lower resolution, as optical path differences in the interferometer, however created, are shorter.

Chao et al have employed liquid crystals (LCs) which, having the largest electro-optic effect of any material, are able to create a relatively large optical path difference within a relatively compact distance, replacing the moving mirror in a standard Michelson based FT spectrometer. An FT spectrometer (see Figure 32) was developed around a $1 \mathrm{~cm}$ long LC clad waveguide to give over $1 \mathrm{~mm}$ optical path difference with no moving parts, and a prototype has been used to measure various absorption features including acetylene gas at $1.540 \mu \mathrm{m}^{[160]}$. The authors suggest that longer waveguides or multipass designs could deliver optical path differences of $1-10 \mathrm{~cm}$ in future devices to give even high spectral resolution.

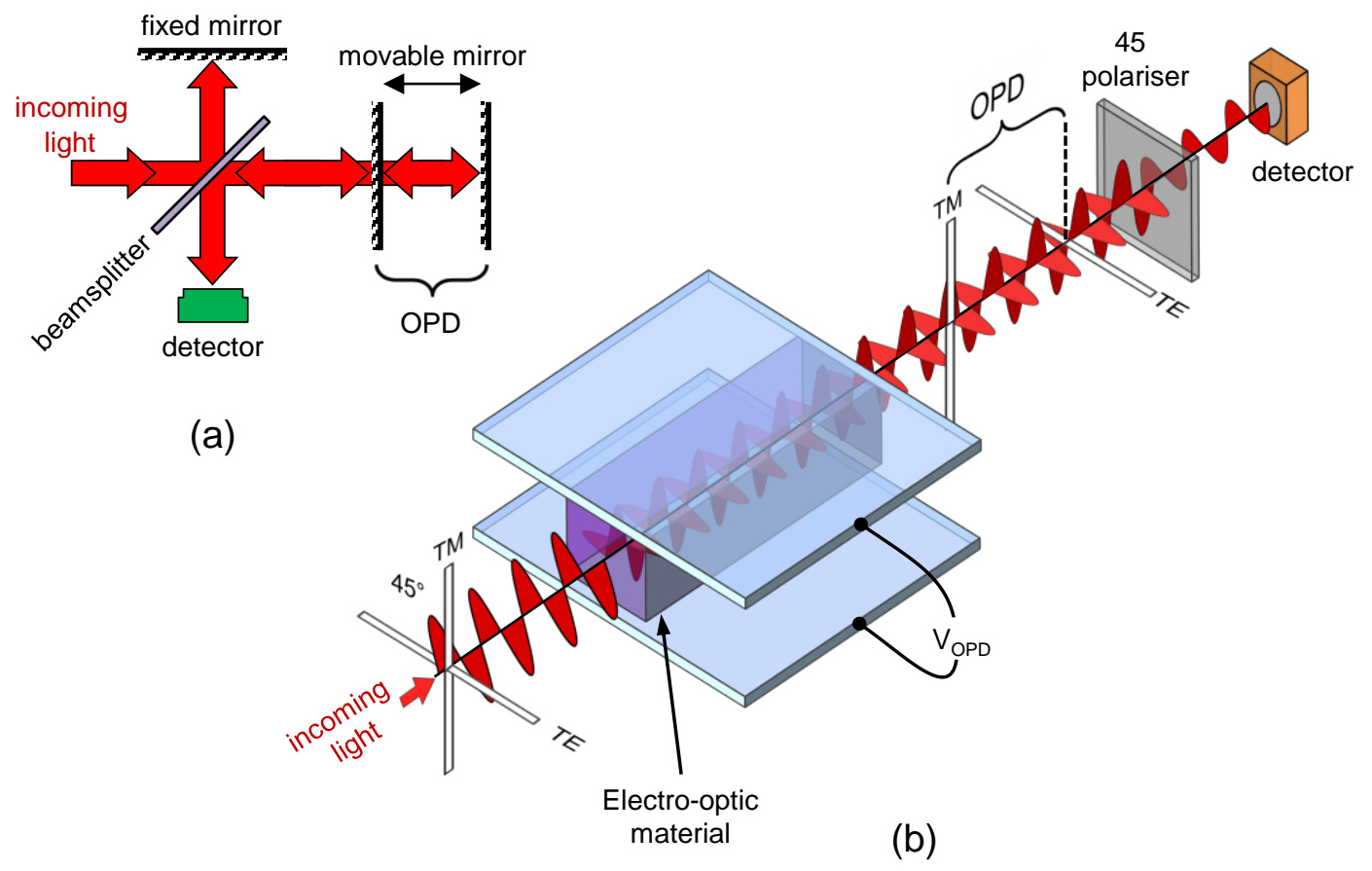

Figure 32. (a) Conventional layout of an FT spectrometer using a Michelson interferometer. (b) Novel introduction of an optical path difference (OPD) using a liquid crystal device. After [160].

Crocombe has analysed the scaling rules for high quality spectrophotometers, governing the effect on apparent source brightness and étendue of a decrease in dimensions of a factor $\kappa^{[161]}$. NIR and UVvisible spectrometers, considered to be shot noise limited, would suffer a degradation in signal to noise ratio by a factor of $\sqrt{ } \kappa$. In contrast, mid IR spectrometers, which are generally limited by detector noise, would suffer a degradation of factor $\kappa$. The most effective mitigation in the mid IR is to use multiplexing techniques (simultaneously detecting light falling into more than one spectral bin at a time $)^{[161]}$. In the UV-visible and near IR, approaches are also based on improving source brightness and / or throughput.

Other limiting factors for spectrometers include "spectral stray light" caused by light scattering from surface imperfections or imperfect light baffling ${ }^{[168]}$ (typically at a level of $0.1 \%$ in commercially available UV-visible diode array spectrometers ${ }^{[162]}$ ), and source flicker noise in incandescent or discharge sources (typically at a level of $\Delta \mathrm{I} / \mathrm{I} \sim 10^{-3}-10^{-5}{ }^{[18]}$ ). If stray light is caused by random scattering, it is likely to increase by a factor of $\kappa^{2}$ in the above analysis, whereas source flicker noise would result in a constant SNR regardless of the spectrometer size. 
Table 7. Comparison of recently developed miniature spectrophotometers. Note that these have not all been used in gas detection.

\begin{tabular}{|c|c|c|c|c|c|c|}
\hline Technical basis & $\begin{array}{l}\text { Wavelength } \\
\text { range } \lambda\end{array}$ & $\frac{\text { Resolution }}{\Delta \Lambda^{\mathrm{a}}}$ & $\frac{\text { Footprint }}{\mathrm{a}, \mathrm{b} /}$ & $\begin{array}{l}\text { Time } \\
\text { constant }\end{array}$ & Limitation & $\underline{\text { Ref }}$ \\
\hline $\begin{array}{l}\text { FT using Wollaston } \\
\text { prisms }\end{array}$ & $200-270 \mathrm{~nm}$ & $0.75 \mathrm{~nm}$ & $\begin{array}{l}\sim 200 \times 200 x \\
400\end{array}$ & $5 s$ & $\begin{array}{l}\text { Benzene } \\
2 \text { ppm.m }\end{array}$ & [163] \\
\hline $\begin{array}{l}\text { DOAS using miniature } \\
\text { diode array }\end{array}$ & 200-850 nm & $2 \mathrm{~nm}$ & 250 & $4 s$ & $\mathrm{NO}_{2} 3 p p b$ & [164] \\
\hline FT with moving grating & $250-800 \mathrm{~nm}$ & $15-21 \mathrm{~nm}$ & $>150$ & Not stated & Not stated & [165] \\
\hline $\begin{array}{l}\text { Spatial heterodyne } \\
\text { interferometry }\end{array}$ & $\begin{array}{l}307-310.5 \\
\mathrm{~nm}\end{array}$ & $0.0058 \mathrm{~nm}$ & $\begin{array}{l}470 \times 360 x \\
240\end{array}$ & $8 s$ & $\begin{array}{l}\mathrm{O}_{3}, \Delta \mathrm{I} / \mathrm{I} \sim \\
0.01-0.003\end{array}$ & [166] \\
\hline $\begin{array}{l}\text { Holographic FT with } \\
\text { wavefront shearing }\end{array}$ & $\begin{array}{l}330 \mathrm{~nm}- \\
1 \mu \mathrm{m}\end{array}$ & $3-25 \mathrm{~nm}$ & $>30$ & Not stated & $\Delta \mathrm{I} / \mathrm{I} \sim 10^{-5}$ & [167] \\
\hline $\begin{array}{l}\text { Grating spectrometer } \\
\text { injection moulded in } \\
\text { polycarbonate }\end{array}$ & $\begin{array}{l}\text { 380-790nm } \\
680-1100 \mathrm{~nm}\end{array}$ & $\begin{array}{l}7-10 \mathrm{~nm} \\
7-8 \mathrm{~nm}\end{array}$ & $\sim 15 \times 50 \times 40$ & Not stated & $\begin{array}{l}\text { Stray light } \\
0.2 \%\end{array}$ & [168] \\
\hline $\begin{array}{l}\text { Dual MEMS grating on } \\
\text { CCD camera }\end{array}$ & 550-750nm & $3 \mathrm{~nm}$ & $\sim 10 \times 20 \times 10$ & Not stated & Not stated & [169] \\
\hline $\begin{array}{l}\text { Linear variable optical } \\
\text { filter on CMOS camera }\end{array}$ & 580-720nm & $2.2 \mathrm{~nm}$ & $\sim 50 \times 50 \times 50$ & Not stated & Not stated & [170] \\
\hline $\begin{array}{l}\text { MEMS tunable Fabry- } \\
\text { Perot filter }\end{array}$ & $1-2.5 \mu \mathrm{m}$ & $0.025 \mathrm{~nm}$ & $27 \times 12 \times 8$ & $4 s$ & $\begin{array}{l}\Delta I / I \sim 2 x \\
10^{-4}\end{array}$ & [156] \\
\hline $\begin{array}{l}\text { Spectral cut-off of tapered } \\
\text { leaky waveguide }\end{array}$ & $\begin{array}{l}1.52-1.62 \\
\mu \mathrm{m}\end{array}$ & $1.5 \mathrm{~nm}$ & $<10$ & Not stated & Not stated & [171] \\
\hline $\begin{array}{l}\text { FT using arrayed } \\
\text { waveguide grating }\end{array}$ & $\begin{array}{l}1.548- \\
1.562 \mu \mathrm{m}\end{array}$ & $0.43 \mathrm{~nm}$ & $16 \times 20$ & Not stated & Not stated & [172] \\
\hline $\begin{array}{l}\text { Liquid crystal waveguide } \\
\text { FTIR }\end{array}$ & $1.45-1.7 \mu \mathrm{m}$ & $\begin{array}{l}3-5 \mathrm{~nm} \text { to } \\
<0.5 \mathrm{~nm}\end{array}$ & $\begin{array}{l}\sim 150 \times 10 \times \\
50\end{array}$ & $\sim 0.5 \mathrm{~s}$ & Not stated & [160] \\
\hline $\begin{array}{l}\text { Dispersed } \\
\text { supercontinuum source }\end{array}$ & $1.1-1.7 \mu \mathrm{m}$ & $0.04 \mathrm{~nm}$ & Not stated & $1 \mathrm{~ms}$ & Not stated & [173] \\
\hline $\begin{array}{l}\text { FT using MEMS } \\
\text { reconfigurable grating }\end{array}$ & $2-4.5 \mu \mathrm{m}$ & $\begin{array}{l}8-40 \mathrm{~nm}^{-1} \\
\left(20 \mathrm{~cm}^{-1}\right)\end{array}$ & $30 \times 30 \times 55$ & $5 s$ & $\begin{array}{l}\mathrm{CO}_{2} \\
60 \mathrm{ppm} \cdot \mathrm{m}\end{array}$ & [174] \\
\hline Standard Michelson FTIR & $2.5-15 \mu \mathrm{m}$ & $\begin{array}{l}1-45 \mathrm{~nm} \\
\left(2 \mathrm{~cm}^{-1}\right)\end{array}$ & $\begin{array}{l}440 \times 310 x \\
190\end{array}$ & Not stated & Not stated & [144] \\
\hline $\begin{array}{l}\text { Spatially modulated prism } \\
\text { interferometer }\end{array}$ & $7.5-12.5 \mu \mathrm{m}$ & $\begin{array}{l}6-16 \mathrm{~nm} \\
\left(1 \mathrm{~cm}^{-1}\right)\end{array}$ & $\begin{array}{l}\sim 300 \times 300 x \\
300\end{array}$ & $\begin{array}{l}\text { Design } \\
\text { only }\end{array}$ & $\Delta \mathrm{I} / \mathrm{I}<0.01$ & [175] \\
\hline \multicolumn{7}{|c|}{$\begin{array}{l}\text { a Figures in italics have been calculated or estimated based on information supplied in each } \\
\text { paper. }\end{array}$} \\
\hline $\begin{array}{l}\text { The footprint of the } \\
\text { appropriate), not ji }\end{array}$ & whole spectro & meter is pro & $\begin{array}{l}\text { ided (including } \\
\text { ement. }\end{array}$ & oupling op & cs where & \\
\hline
\end{tabular}

A number of alternative spectrometer designs is compared in Table 7. What is apparent is the diversity of technological approaches and of performance specifications in this field, resulting from a great variety of applications for field based spectrophotometry. For example, a high resolution spectrometer 
has been configured for measurement of ozone with a spectral range of 307-310.5 nm and resolution of $0.0058 \mathrm{~nm}^{[166]}$, fully resolving the gas absorption lines but with no ability to measure other gases. In contrast, a micro FT spectrometer with a range of $2-4.5 \mu \mathrm{m}$ has a resolution of $8-40 \mathrm{~nm}^{[174]}$. Taking $\mathrm{CO}_{2}$ detection as an example, this would be insufficient to resolve the individual gas lines, but with higher effective resolution than the bandwidth of filters used in NDIR, sufficient to measure a number of gas absorption bands. There are clearly compromises to be made between the need to identify individual species, the number of gases to be detected and limits of detection for those gases.

Dell et al have pointed out that for many industrial spectrometer applications, high resolution is not necessarily required ${ }^{[176]}$. For many liquid or solid phase samples, measurement of individual species is not needed; rather one requires quantification of some derived parameter such as classification of crude oil, or total oxygen demand. An equivalent example in gas analysis might be measurement of calorific value of natural gas mixtures. Dell et al claimed that use of low resolution spectra (insufficient to resolve spectral features) combined with chemometric techniques can deliver adequate performance in these applications ${ }^{[176]}$.

\section{Tunable diode laser spectroscopy}

With tunable diode laser spectroscopy (TDLS), the emission wavelength of a narrow linewidth laser diode is scanned across an individual gas absorption line at very high resolution ${ }^{[177]}$. The measurement is effectively self-referenced by comparing the central peak absorption to the zero level on either side of the line. Working at such high resolutions therefore gives the following advantages:

- High signal to noise ratios resulting from fully resolved gas lines and a narrow effective baseline.

- A high degree of specificity to the target gas, as long as a region can be found for which the narrow individual absorption line of the target has no overlap with lines of potential interferents. This can often be achieved for small molecules, even for species within the same family (for example detection of methane against a background of other hydrocarbons ${ }^{[10]}$ ).

- Fast operation; the wavelength of many diode lasers can be modulated at frequencies up to $\mathrm{MHz}$, however commonly used signal recovery electronics is often limited to around $100 \mathrm{kHz}$.

TDLS has been comprehensively reviewed elsewhere ${ }^{[1,178,179]}$, therefore the purpose of this section is to bring these reviews up to date with recent developments.

\subsection{Basic principles of TDLS}

For simple, single pass or folded path gas cells there are two commonly used techniques for TDLS, termed direct spectroscopy or line scanning, and wavelength modulation spectroscopy. Instruments based on both these techniques have been commercialised ${ }^{[180]}$. A general schematic that includes both approaches is given in Figure 33. In standard laser diodes, typically distributed feedback (DFB) lasers or vertical cavity surface emitting lasers (VCSELs), the emission wavelength is tuned by changing the temperature of the laser cavity over a few seconds via a Peltier element or more rapidly via the injection current at modulation rates that can extend into the $\mathrm{MHz}$ regime ${ }^{[181]}$. The latter also has the (usually undesirable) result of increasing the emitted power, known as residual amplitude modulation (RAM), shown in Figure 33(a). 


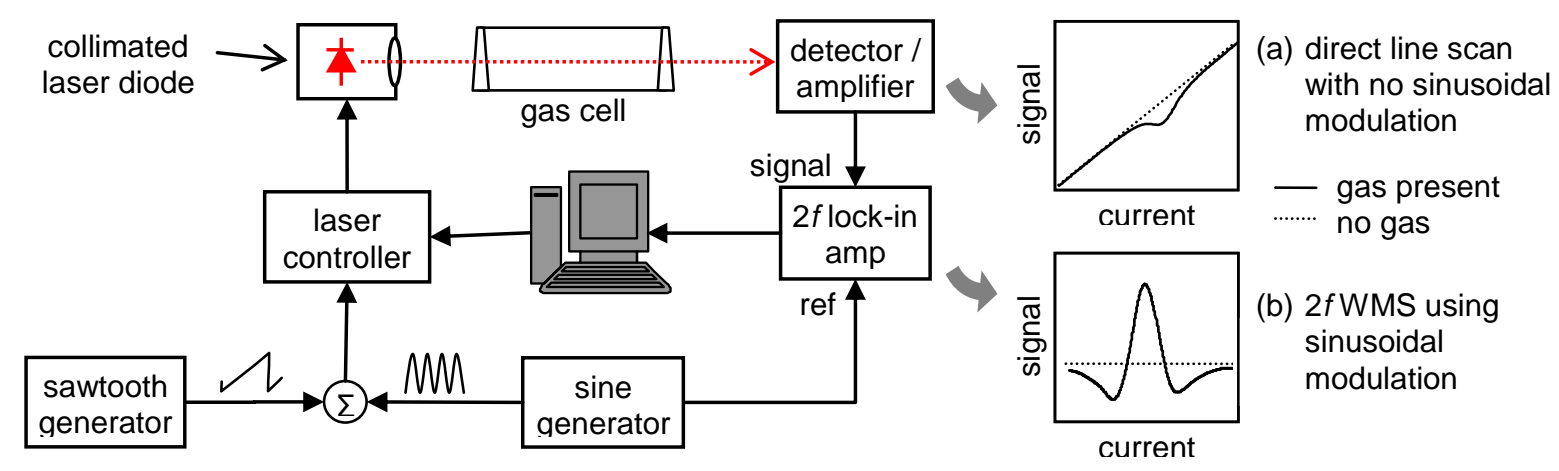

Figure 33. Conventional configuration for TDLS employing a laser diode, showing detected signals as a function of laser drive current for (a) direct scanning of the gas line, or (b) $2^{\text {nd }}$ harmonic wavelength modulation spectroscopy ( $2 f$ WMS). The effects of residual amplitude modulation can be seen in the rising background signal in plot (a).

Direct spectroscopy involves scanning the output of a laser diode across one or more gas lines in a narrow range, by ramping the laser diode injection current. The resulting transmission shows a rising background (the laser's output intensity increases with drive current) with a dip corresponding to the gas line absorption, as shown in Figure 33 (a). By carefully subtracting a zero reference, or by taking a ratio of the detected signal to the input light intensity, the gas absorption alone can be recovered. The potential for multi - gas detection is illustrated by a scan taken from a tunable DFB laser diode in Figure 34.

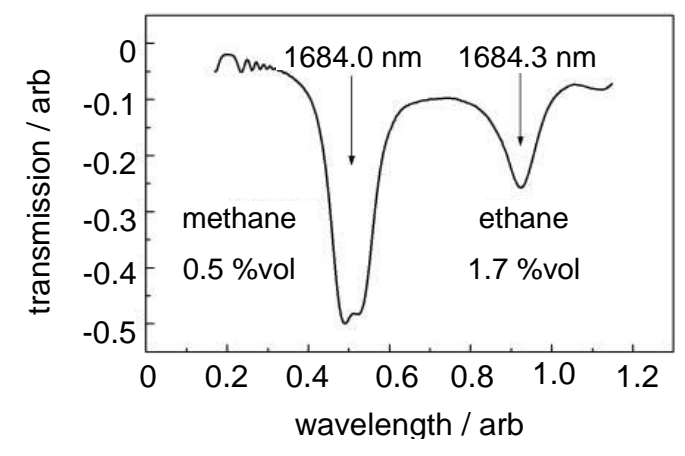

Figure 34. Example of a dual gas measurement of methane and ethane using a wavelength scan from a single DFB laser. Taken from reference [182].

It is common to use a spectral fitting technique (typically the Marquardt - Levenburg algorithm ${ }^{[183]}$ ) to determine the gas concentration from a line trace. Spectral fitting allows several gas lines to be separated from a single trace, and has for example been used to detect methane and ethane levels in natural gas, with a limit of detection for methane at the ppm level using a $20 \mathrm{~cm}$ pathlength ${ }^{[184]}$.

Using wavelength modulation spectroscopy (WMS), an AC modulation signal is applied to the laser diode, giving a sinusoidal modulation of the emission wavelength that acts as a dither over absorption features ${ }^{[177]}$. The method confers two advantages: an improved signal : noise ratio resulting from improved sensitivity to curved spectral features of the appropriate width, and a zero baseline, improving zero stability. Gas is typically detected by recovering the $2^{\text {nd }}$ harmonic of the AC excitation 
at the receiver with a lock-in amplifier, as shown in Figure 33. The typical forms of different demodulated harmonics are shown in Figure 35 for a single gas line.
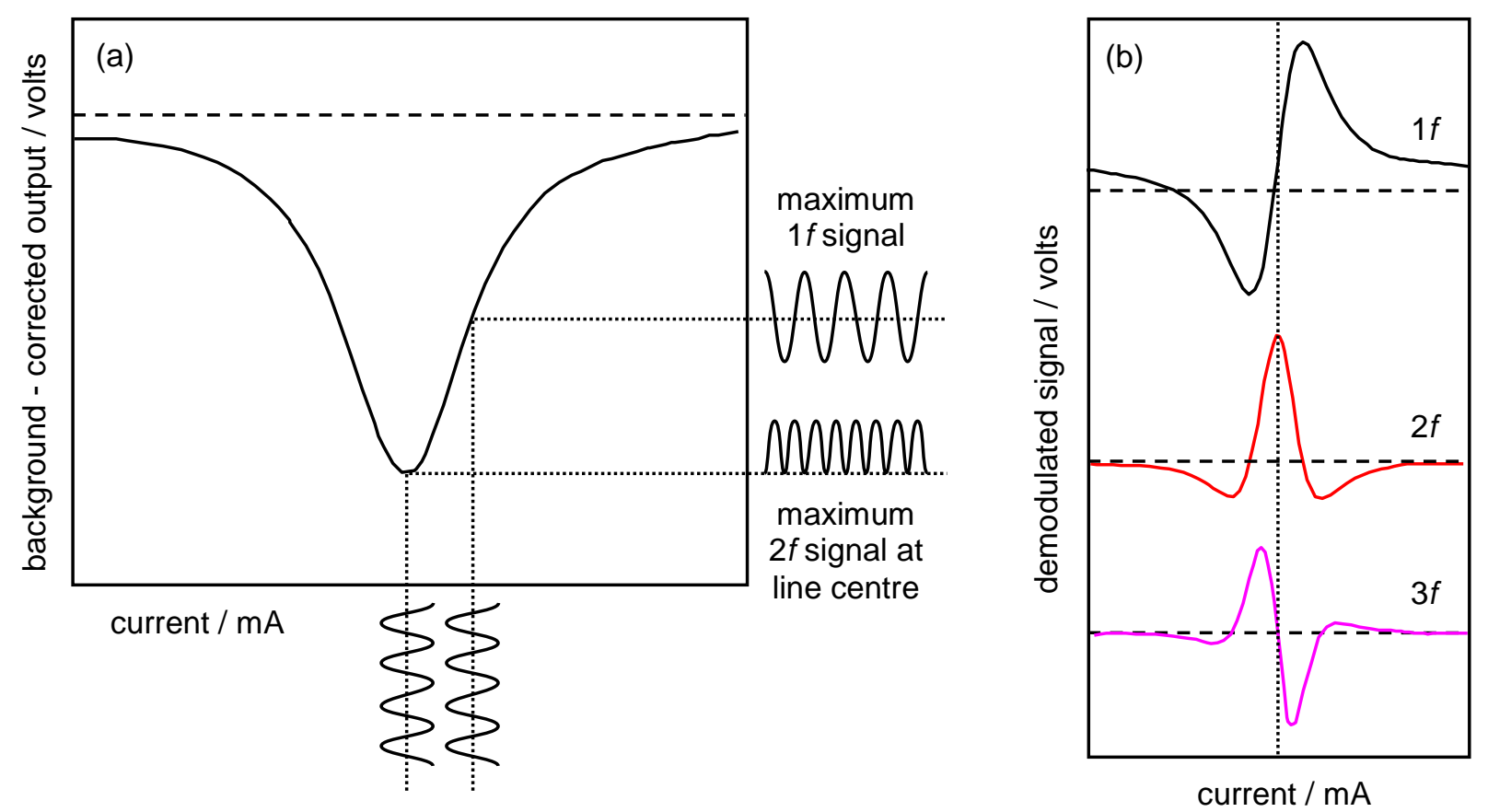

Figure 35. Generation of different harmonic signals in wavelength modulation spectroscopy (WMS).

(a) DC scan through a single gas line, background-corrected to remove the residual amplitude modulation for clarity. (b) Form of $1 f, 2 f$ and $3 f$ harmonics as a function of position in the current scan, displaced for clarity. The RAM creates a small but measurable DC offset on the $1 f$ signal.

The position of the absorption line in the trace can vary with operating conditions. It is not the gas absorption wavelength that varies, but the mean emission wavelength of the laser diode, which cannot be determined with sufficient precision to enable wavelength control, other than by reference to the position of the gas line. (Indeed, gas lines are often used as wavelength standards in telecommunications ${ }^{[185]}$.)

The odd harmonics are antisymmetric about the line centre (see Figure 35). A PID feedback loop using the $1^{\text {st }}$ or $3^{\text {rd }}$ harmonic to provide an error signal ensures that the emission wavelength remains locked to the gas absorption line. The $1^{\text {st }}$ harmonic has a "DC offset", caused by RAM, that must be corrected for. This offset is absent (to first order) in the $3^{\text {rd }}$ harmonic, making this harmonic more robust as an error signal, a particularly valuable feature for systems subject to a wide dynamic range in the level of the returned signal and / or variable levels of DC background ${ }^{[186]}$. However, $3^{\text {rd }}$ harmonic demodulation is rarely implemented in low cost off-the-shelf lock-in amplifiers.

Alternatively, a slow ramp may be added to the sinusoidal dither to provide a scan of the $2 f$ signal over the region of the gas line. For some measurements, the target line's position in the scan may be determined by reference to a neighbouring line of a different gas, whose presence can be guaranteed ${ }^{[187]}$. Line fitting algorithms are available to determine the concentration from the entire lineshape rather than just its peak value ${ }^{[188]}$. Providing a line scan rather than a single peak value can 
improve SNRs by enabling post-detection filtering of interference fringes with FSRs that are much narrower or wider than the gas linewidth.

To optimise WMS, the so-called modulation index (ratio of the modulation amplitude to the gas linewidth) is typically set equal to 2.2 for recovery of the $2 f$ signal from a Lorentzian line profile ${ }^{[189]}$. Use may also be made of the phase difference between the $2 f$ signals due to RAM and to gas absorption, in order to recover the former in the absence of the latter ${ }^{[190]}$. This can enable complete recovery of the gas lineshape in the face of variable pressure and temperature. Otherwise, sensor calibration demands knowledge of these parameters plus the characteristic response of the laser diode, which can vary from device to device. Chen et al have described a set of algorithms for determining the required parameters in situ, avoiding frequent instrument calibration ${ }^{[191]}$.

\subsection{Limitations and improvements to TDLS}

Detection sensitivity in TDLS is often limited by optical interference fringes rather than the theoretical limit given by detector noise ${ }^{[192,193]}$. The fringes stem from Fabry-Perot etalons between reflecting or scattering surfaces such as mirrors, lenses, optical fibre end faces, detector and laser head windows, semiconductor surfaces, and components of multipass cells ${ }^{[177]}$, and also from low levels of optical feedback to the laser diode ${ }^{[194]}$. Gas absorption linewidths are narrow (full width at half maximum of $\sim 5 \mathrm{GHz}$ at atmospheric pressure) and therefore to resolve the gas line requires the use of lasers with narrower emission linewidths; tens of $\mathrm{MHz}$ is typical, and more than adequate for pressure broadened gas lines. One consequence of the narrow linewidth is the long coherence length of the laser emission (typically tens of metres), which means that these interference effects are unavoidable for such high resolution spectroscopy.

The gas sample cells themselves have been considered to be frequent culprits ${ }^{[22]}$, because typical lengths of the order of $30 \mathrm{~mm}$ give rise to fringes with a free spectral range of the order of $5 \mathrm{GHz}$. Despite the use of wedged windows and antireflection coated optics, small Fresnel reflections often persist in the optical path ${ }^{[177]}$ or develop after a period of time in the field ${ }^{[44]}$. Interference can also result from diffuse reflections from light scattering materials ${ }^{[194]}$, suggesting that dust or dirt could also cause problems. Over time, fluctuations in temperature and / or vibrations of the cavity cause the interference fringes to shift in wavelength such that they cannot be removed by subtraction of a zero baseline.

Techniques to reduce the effects of interference fringes include:

- Wedging and angling of all windows, antireflection (AR) coating of windows and lens surfaces, and angle polishing optical fibre ends. As long as the incident light is highly polarised, Brewster angle windows may be used. Reflections from each optical surface must be misaligned from the optical path.

- Removing the windows altogether, from both the gas cell and from the laser diode and detector packages. This requires very careful sample conditioning to ensure that water vapour and especially condensation do not reach the laser diode.

- Filtering out fringes by applying a higher frequency jitter to the laser diode and integrating the signal ${ }^{[195]}$, or post detection filtering using high pass filters ${ }^{[177]}$, low pass filters ${ }^{[196]}$ or Fourier domain analysis ${ }^{[197]}$. However, neither approach can deliver an improvement in signal to noise ratios for fringes whose FSR is comparable to the gas absorption linewidth ${ }^{[177]}$. 
- Mechanically modulating the fringe spacing and integrating the signal. This has been implemented using longitudinal dithering of optical elements ${ }^{[22]}$, an oscillating mirror ${ }^{[192,198]}$ and an oscillating Brewster-plate spoiler ${ }^{[199]}$. While effective, this approach limits the available detection bandwidth and adds to system complexity.

- For fringes that result from optical feedback into the laser cavity, the effects can be reduced using optical isolators, but at custom wavelengths these can cost more than the laser diode itself. A feature of this form of interference is that it results in a modulation of the entire laser diode output, so that its effect can also be reduced by subtraction of an intensity reference ${ }^{[194]}$, as implemented in balanced detection schemes.

To improve limits of detection, it is possible to use more advanced techniques. Operation at reduced pressure can improve the limit of detection in the following way, for a given gas concentration. In conditions dominated by pressure broadening of the gas lines, the absorption linewidth according to equations (3) and (4) is approximately proportional to pressure. This has the effect of reducing the width of the spectral baseline required to self-reference the measurement, and of shifting the interference problem towards fringes with a reduced FSR (and longer cavity length). Both effects lead to improvements in the limit of detection; for example, Engelbrecht has estimated an improvement factor of 7 for a pressure reduction from $1 \mathrm{~atm}$ to $0.08 \mathrm{~atm}$ (by a factor of 125) ${ }^{[200]}$.

Balanced detection schemes have been used not only to reduce the effects of optical feedback, but also to provide first order reduction of intensity noise and other fluctuations. Engelbrecht used such a scheme to detect absorbances as low as $6 \times 10^{-7} \mathrm{~Hz}^{-1 / 2}$ for WMS measurements using the configuration shown in Figure $36^{[200]}$. Brewster angle windows were used for the gas cell, requiring the use of polarisation maintaining fibre between the spectrometer and the measurement location.

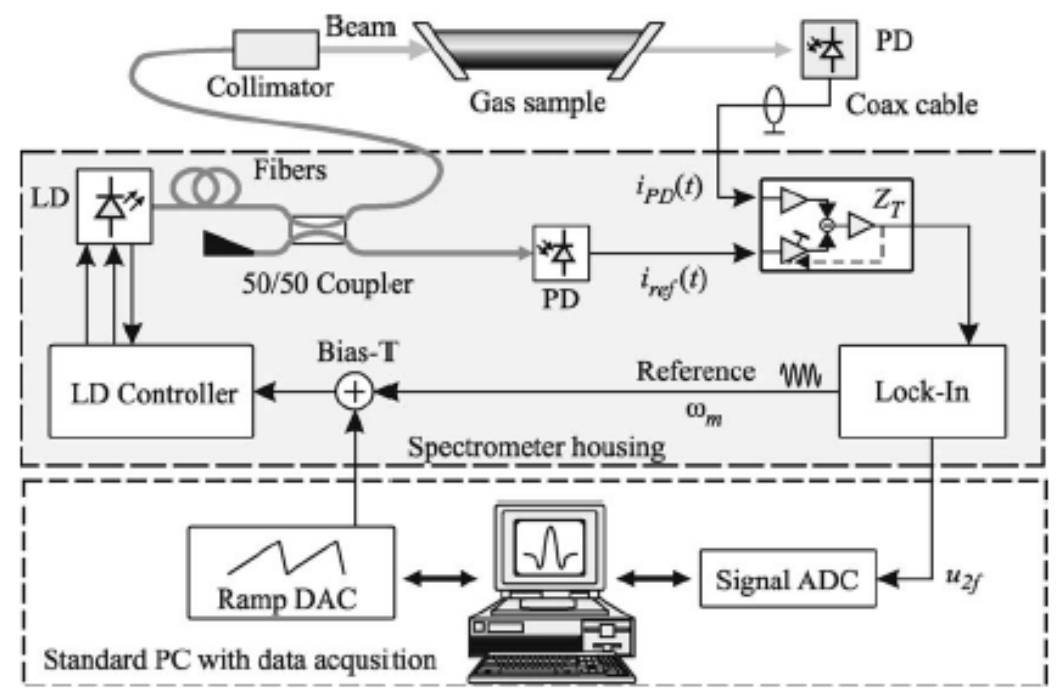

Figure 36. Configuration for WMS based measurement of $\mathrm{CO}$ and $\mathrm{CO}_{2}$ using a balanced receiver, taken from [200].

High frequency WMS makes use of the fact that the laser excess noise is reduced at higher frequencies, often obeying a $1 / f$ characteristic at low frequencies ${ }^{[201]}$, as shown in Figure 37 . If the signal : noise ratio for the measurement is to be improved, other sources of noise and uncertainty, such as interference fringes, must be reduced below the level of laser excess noise. For applications involving low levels of returned power and therefore high gain pre-amplifiers at the detector, the 
operating frequency may be limited by the detector bandwidth to tens of $\mathrm{kHz}$. For WMS, many commercial lock-in amplifiers are often limited to a maximum operating frequency of around $100 \mathrm{kHz}$; note that this determines the maximum frequency of the highest recovered harmonic.

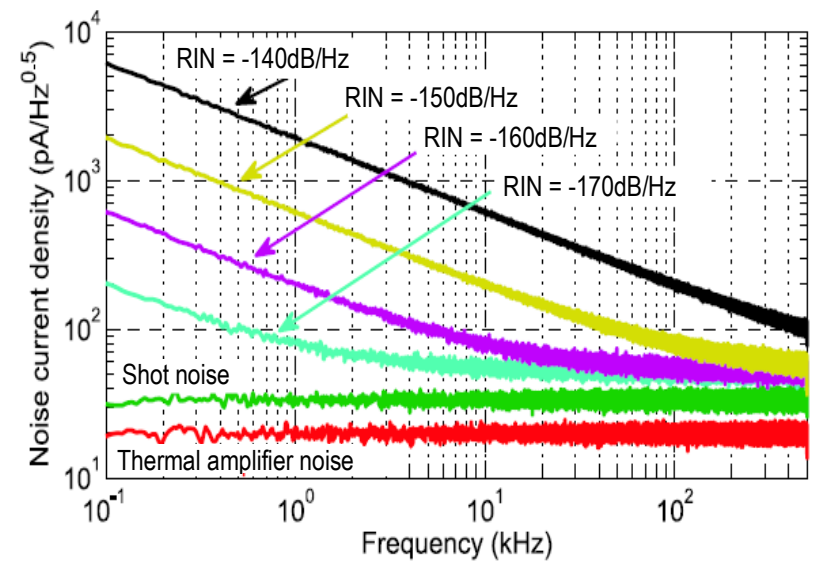

(a)

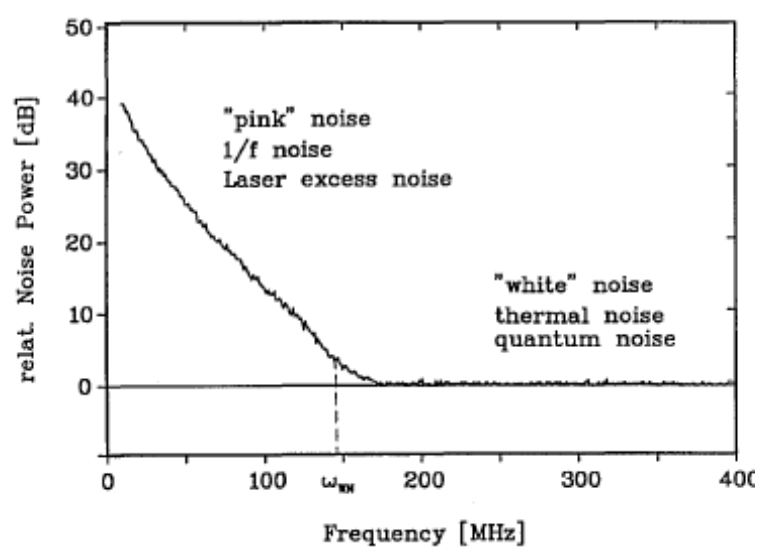

(b)

Figure 37. Relative Intensity Noise (RIN) of laser diodes, showing a $\sim 1 / \mathrm{f}$ noise characteristic at low frequencies and white noise thereafter. (a) Simulated noise current density of DFB lasers, supported by measurements on a single diode (NEL NLK1556STB) with a RIN of $-140 \mathrm{~dB} / \mathrm{Hz}$, taken from [201].

(b) Noise frequency spectrum of a lead salt diode laser, taken from [31].

Frequency Modulation Spectroscopy (FMS) uses modulation at radio frequencies (RF), typically hundreds of $\mathrm{MHz}$. Detector-limited sensitivities of the order of $10^{-7}$ to $10^{-8}$ have been achieved ${ }^{[196,202]}$. Early work with lead salt diode lasers demonstrated that increasing the modulation frequency from $2 \mathrm{MHz}$ to $200 \mathrm{MHz}$ could improve the SNR by several orders of magnitude ${ }^{[31]}$. For such lasers the RIN obeyed a $1 / f$ noise characteristic for frequencies up to the order of $100 \mathrm{MHz}^{[31]}$, therefore noise was minimised by operation at frequencies in the hundreds of $\mathrm{MHz}$ range. However, as Figure 37 shows, there may be little or no benefit for more recently available room temperature DFB lasers in the use of such high frequencies.

Further developments are driven by the availability of high performance personal computers and the opportunities provided by digital signal processing. A PC based TDLS system has been implemented with four lock-in amplifier channels, with performance claimed to be equal to that of a standard system based around discrete lock-in amplifiers ${ }^{[203]}$. Various groups have developed board-level systems with $2 f$ WMS and line scanning, laser control, $2 f$ demodulation and curve fitting for standalone sensors, including oxygen sensors ${ }^{[187,204]}$, and methane / ethane ${ }^{[184]}$. Using arbitrary waveform programming, an optimal modulation signal has been devised for WMS that maximises the time spent sampling the most important elements of the spectrum in the curve fit, to give a twofold improvement in SNR ${ }^{[205]}$.

\subsection{Tunable lasers}

\subsubsection{Solid state, monolithic devices}

In this section we consider laser diodes and their mid IR counterparts (which are not strictly diodes but can be operated in a similar manner). Typically in the near infrared, distributed feedback (DFB) diode lasers or vertical cavity surface emitting lasers (VCSELs) are used. Each must be custom made at a 
particular wavelength (the specific gas absorption line). Wavelength selection is required for mode-hop free singlemode operation; DFB lasers employ a grating structure, usually written above the active waveguide region, whereas VCSELs typically employ interference layers above and below the active layers. Custom lasers are required for any gases whose absorption lines do not overlap with the 1.3 or $1.55 \mu \mathrm{m}$ telecommunications bands (these bands cover weak $\mathrm{CO}$ and $\mathrm{CO}_{2}$ lines, $\mathrm{H}_{2} \mathrm{~S}$ and $\mathrm{NH}_{3}$, but not the hydrocarbons, $\mathrm{NO}$ or stronger $\mathrm{CO}$ and $\mathrm{CO}_{2}$ lines). Only a limited range of such wavelengths is routinely available, and these custom devices are often the most expensive component for industrial systems.

A number of attempts have been made to develop an alternative. Multimode absorption spectroscopy ("MUMAS") proposes the use of simpler, low cost multimode (Fabry-Perot) laser diodes, allowing multiple emission modes to be absorbed by gas lines as they happen upon them ${ }^{[206]}$. The result is a convolution of the emission and absorption spectra, yielding useful information when spectra are relatively sparse. Figure 38 shows examples of the resulting spectra for acetylene. For the spectra to remain stable these laser diodes must be made with reduced gain in the cavity ${ }^{[207]}$.

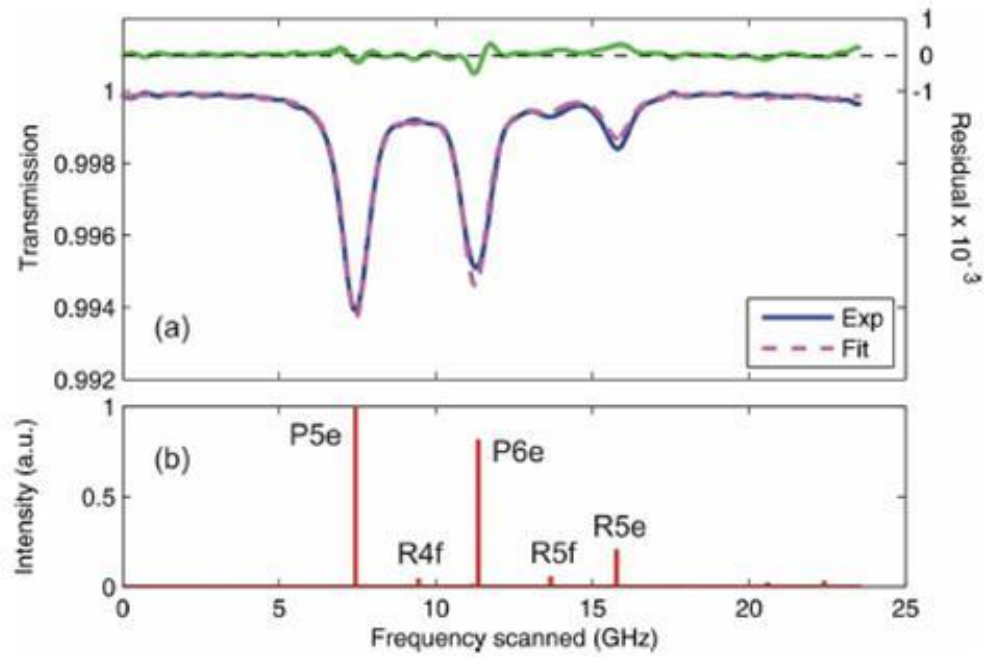

Figure 38. MUMAS spectra recorded around $1.529 \mu \mathrm{m}$ for acetylene at a pressure of 10 Torr. The upper line shows the difference between the experimental and fitted curves. Taken from [206].

Because the absorption lines for a given gas can be of the order of $100 x$ stronger in the mid IR, there has been significant activity in recent years to develop a practical, room temperature tunable laser diode to cover this region. Unfortunately, conventional semiconductor technology, in which the emitted wavelength corresponds to a transition within a bandgap in the gain medium, reaches a thermal limit at longer wavelengths, whereby bandgap energies approach those of background thermal fluctuations in the material. Therefore until some years ago, the only commercially available devices were cryogenically cooled lead salt laser diodes. Nevertheless, longer wavelength extension of near IR wavelengths of standard semiconductor diode VCSELs and quantum well (QW) DFB lasers has resulted in emission at wavelengths up to $2.6 \mu \mathrm{m}^{[208]}$ and $3.4 \mu \mathrm{m}^{[209,210]}$ respectively. And the recent success of several alternative approaches has now resulted in almost complete coverage of the mid IR region with "turnkey" tunable lasers operating at close to room temperature (see Figure 39). 


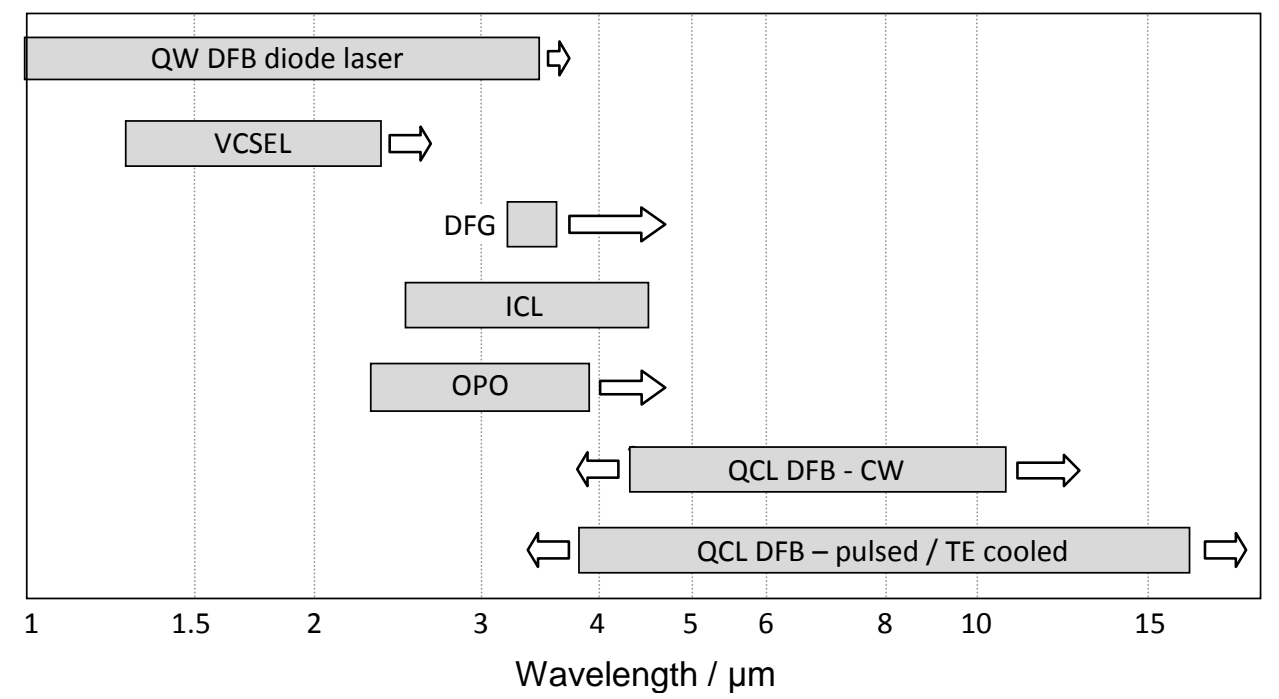

\begin{tabular}{|l}
\hline $\begin{array}{l}\text { Frankfurt, Laser Components, } \\
\text { Nanoplus, NEL, Roithner } \\
\text { Vertilas } \\
\text { Novawave (Thermo Fisher) }\end{array}$ \\
Maxion \\
Lockheed Martin \\
$\begin{array}{l}\text { Adtech, Alpes, Daylight } \\
\text { Solutions, Frankfurt, } \\
\text { Hamamatsu, Laser Compo- } \\
\text { nents, Maxion, Roithner }\end{array}$ \\
\hline
\end{tabular}

Active suppliers

Key CW: continuous wave, QW DFB: quantum well distributed feedback, DFG: difference frequency generation, ICL: interband cascade laser, OPO: optical parametric oscillator, QCL: quantum cascade laser

Figure 39. Wavelength coverage at the time of writing for "turnkey", room temperature, solid state tunable lasers in the IR (above $1 \mu \mathrm{m}$ ). Arrows indicate the direction of research activity in a rapidly developing field. The list of suppliers may not be exhaustive and does not include those supplying exclusively in the telecommunications bands (around $1.3 \mu \mathrm{m}$ and $1.55 \mu \mathrm{m}$ ).

Quantum cascade lasers (QCLs) have been widely commercialised in both pulsed and CW forms, and have been recently reviewed by Capasso ${ }^{[211]}$. Artificially generated energy levels are created via nanometre scale quantum well structures, providing transitions that are independent of the material bandgap. Each electron passes through the cascade structure shown in Figure 40, emitting a photon at each stage ${ }^{[212]}$. Decoupling the emission wavelength from the bandgap has allowed QCLs to be developed over a very wide range of wavelengths from the mid IR to the terahertz $(\mathrm{THz})$ region, however their use for gas sensing in the $\mathrm{THz}$ region has been limited ${ }^{[211]}$. As with semiconductor laser diodes, DFB structures are often used to ensure singlemode operation. Output powers can be significantly higher than with alternative lasers (hundreds of $\mathrm{mW}$ for commercially available, room temperature $\mathrm{CW}$ devices), necessitating greater attention to heat dissipation, but making them more suitable to applications that benefit from higher power such as photoacoustic or backscatter detection $^{[213]}$. The appendix shows numerous examples of the use of QCLs for detection of different gas species; minimum detectable absorbances better than $10^{-6}$ are achievable ${ }^{[387]}$. 


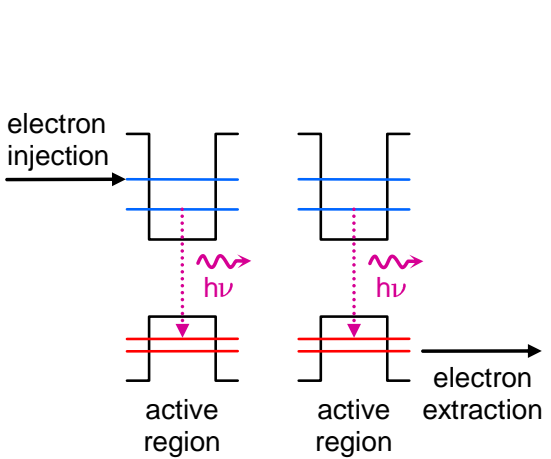

(a) Diode lasers

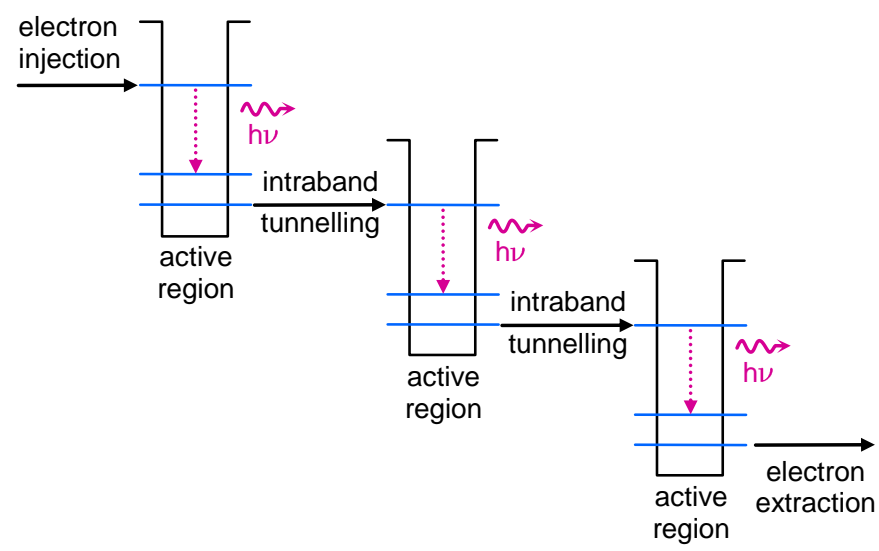

(b) Quantum cascade lasers

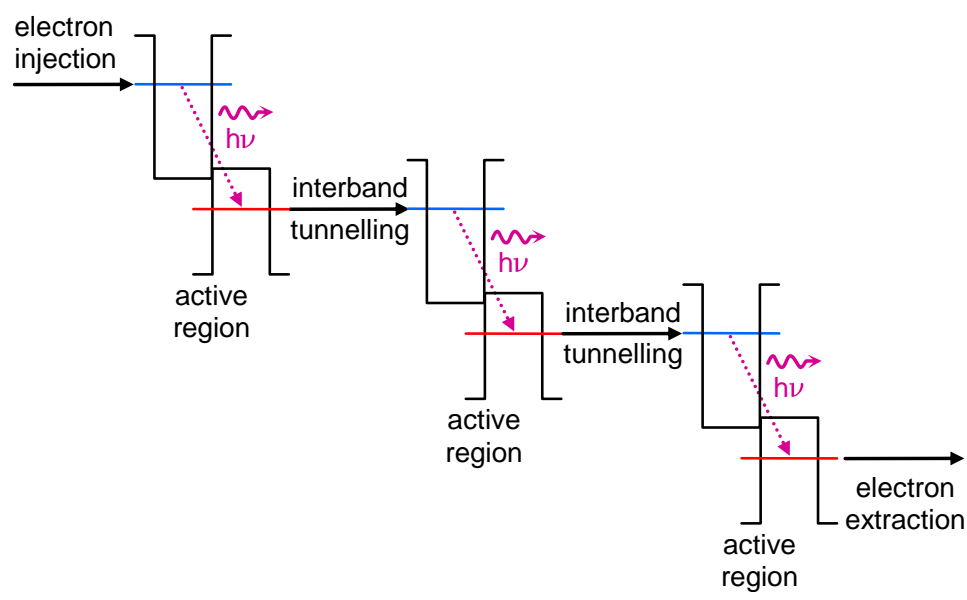

(c) Interband cascade lasers

Figure 40. Comparison of energy levels and active region processes for (a) diode lasers, (b) quantum cascade lasers and (c) interband cascade lasers. After [213].

Interband cascade lasers (ICLs) cover an important gap in wavelength coverage between diode lasers and QCLs with a standard operating range between 2.5 and $4.4 \mu \mathrm{m}$. This wavelength region covers many interesting species as it includes fundamental $\mathrm{C}-\mathrm{H}, \mathrm{O}-\mathrm{H}$ and $\mathrm{N}-\mathrm{H}$ stretch vibrations. The $\mathrm{ICL}$ operating principle can be considered a hybrid of the conventional diode and $\mathrm{QCL}$, and output powers are of the order of tens of $\mathrm{mW}^{[214]}$. Both pulsed and $\mathrm{CW}$ versions available, and fabrication procedures have been developed to create DFB structures for mode-hop free singlemode operation. Because of the relative immaturity of ICLs compared to their QCL counterparts there have been fewer instrument developments based around them, however NEAs of $10^{-4}-10^{-5}$ are achievable ${ }^{[215]}$.

\subsubsection{Widely tunable laser systems}

External cavity lasers have long offered narrow linewidth, singlemode operation with tuning over a wide range ${ }^{[216]}$. In external cavity diode lasers $(E C D L)$, a diode is used as the gain medium, often specifically engineered for use in an ECDL; such devices can be obtained commercially as "gain chips", especially in the near IR spectral region. The external cavity then typically incorporates a separate wavelength-selective element, often a grating, in the Littrow ${ }^{[217]}$ or Littman-Metcalf ${ }^{[218]}$ configurations, illustrated in Figure 41. The Littrow configuration is the simplest in that it involves fewer 
optical elements and less initial alignment. However, the Littman-Metcalf configuration has the advantage of avoiding beam deflection during tuning.

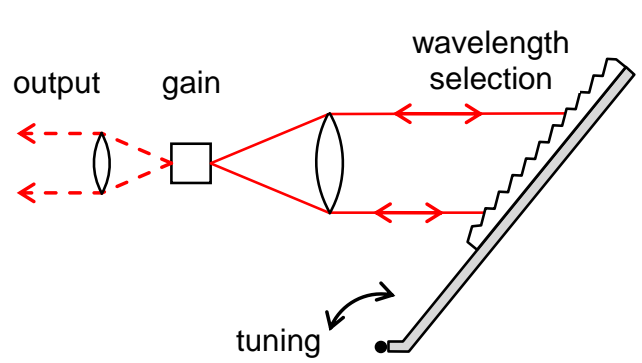

(a) Littrow

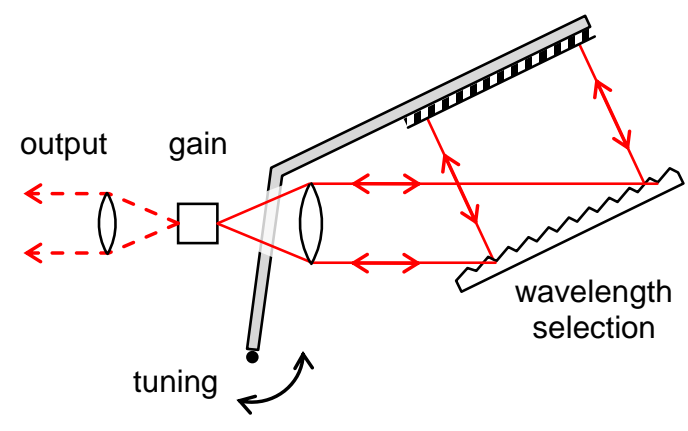

(b) Littman-Metcalf

Figure 41. External cavity diode lasers in standard Littrow or Littman-Metcalf configurations, using mechanically rotated gratings to provide wavelength tuning. Here, the emission is from the rear facet of the gain medium, however it can also be obtained from the zero order beam reflected from the grating.

Recently there has been considerable activity to reduce the size of these lasers using MEMs technology, as reviewed by Liu and Zhang ${ }^{[219]}$. Singlemode tunable lasers have been realised using both Littrow and Littman-Metcalf schemes. As well as providing compactness for its own sake, this also has the advantage of enabling very short cavities, such that the wavelength selective element can more easily select a single cavity mode. However, Liu and Zhang noted that developments in packaging and device integration may be needed before many of these designs are used in real applications $^{[219]}$.

By adding a MEMS-actuated mirror to the top of a standard VCSEL, a group at TU München has increased the laser's tuning range to between 20 and $60 \mathrm{~nm}^{[220]}$. This would enable a "useful" range of gas detection wavelengths to be addressed using a smaller number of commercial products, which could increase effective production yields.

The QCL operating principle allows the stacking of cascade regions with different emission wavelengths to create broadband gain within a single device. These are suitable for use in external cavity lasers with wide tuning that cover a range of different gas species ${ }^{[21]}$, and turnkey systems have been commercialised by several companies ${ }^{[222]}$. Hugi et al have demonstrated a single device capable of being tuned from 7.4 to $11.6 \mu \mathrm{m}\left(432 \mathrm{~cm}^{-1}\right)$ with an average output power of $15 \mathrm{~mW}$ and linewidth below $2 \mathrm{~cm}^{-1}[223]$. An external cavity $\mathrm{ICL}$ has also been demonstrated, with a tuning range from 3.13 to $3.24 \mu \mathrm{m}\left(110 \mathrm{~cm}^{-1}\right)$, and output power of 1 to $4 \mathrm{~mW}$ across the tuning range ${ }^{[224]}$. These systems therefore have the potential to replace FTIR spectrometers for certain applications requiring a reduced wavelength range.

The widest mode-hop free tuning range in the mid IR (around $1 \mu \mathrm{m}$ tuning, dependent on configuration $^{[225]}$ ) can often be achieved by using either of two nonlinear wavelength conversion schemes, in difference frequency generation (DFG) systems and optical parametric oscillators (OPOs). The key to this process is an optical crystal with a high nonlinear coefficient, which is transparent at the wavelengths concerned, such as lithium niobate $\left(\mathrm{LiNbO}_{3}\right)$. However, $\mathrm{LiNbO}_{3}$ is only 
transparent up to $5 \mu \mathrm{m}$, limiting its range of applicability and therefore interest has also grown in the use of alternative materials such as GaAs, with a transmission window from $0.9-17 \mu \mathrm{m}^{[226,227]}$.

DFGs systems have been reviewed elsewhere by Richter et al ${ }^{[228]}$. In general, the sources consist of two seed lasers - a pump (high power) at frequency $\nu_{p}$ and a tunable source (lower power) at $\nu_{s}$, which are combined to produce an idler output at $\nu_{\mathrm{i}}=\nu_{\mathrm{p}}-\nu_{\mathrm{s}}$ by conservation of energy (see Figure 42). For efficient nonlinear conversion, conservation of momentum also requires phase matching of the pump, signal and idler beams. The recent success of DFG based spectrometers owes much to developments in periodically poled lithium niobate (PPLN). These materials allow quasi phase matching schemes involving different grating periods for the pump, signal and idler beams, and also have the advantage of easier alignment than non periodically poled alternatives ${ }^{[25]}$. Quasi phase matching can be maintained over a wide tuning range by changing the temperature of the crystal while simultaneously tuning the source.

DFG sources are limited in their output power by the damage threshold of the crystal and other effects $^{[229]}$, however powers of up to tens of $\mathrm{mW}$ can be achieved ${ }^{[230]}$. DFG sources are particularly suited to the 3-4 $\mu \mathrm{m}$ region, which has been difficult to access with their main rivals, QCLs (see Figure 39). To achieve a wide IR tuning range requires a widely tunable near IR source; fortunately, many suitable sources are available in the $1.55 \mu \mathrm{m}$ near IR telecoms band. Fischer and Sigrist used a 1.51.6 $\mu \mathrm{m}$ ECDL source to provide continuous tuning of the idler in the range $3.2-3.7 \mu \mathrm{m}$, and used this in combination with photoacoustic detection (see section 8) to record spectra for methane, ethane and hydrogen chloride with low ppm level detection limits ${ }^{[231]}$.

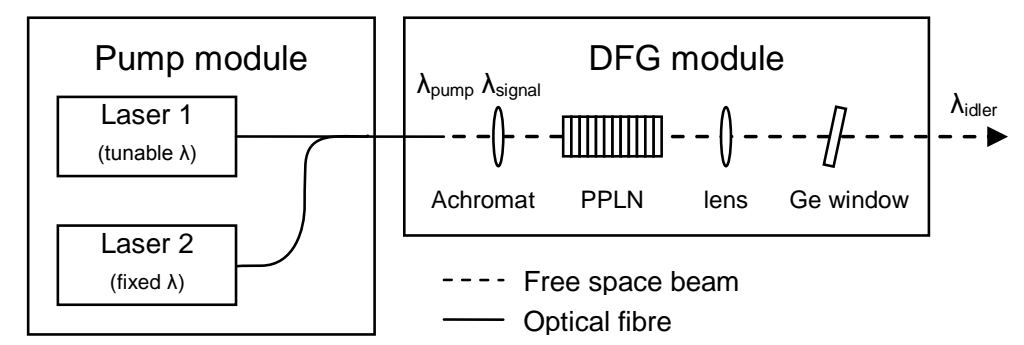

Figure 42. Schematic diagram of a difference frequency generation (DFG) source using periodically poled lithium niobate (PPLN). After [229].

The need to realign the crystal and other components in these systems, as well as the physical size of the system compared to discrete laser devices, has precluded the use of DFGs in many field applications, however there are developments aimed at improving reliability and field robustness. By analysing various elements of the optical alignment in their system, Richter et al were able to show an NEA of $3 \times 10^{-7}$ in the laboratory (with 10 minute averaging), deploying on an aircraft in a field campaign with a 10 -fold deterioration in performance ${ }^{[228]}$. Cousin et al have also built a field deployable DFG employing telecommunications sources and fibre (although the PPLN crystal was still a free space component) and used this with a multipass cell at $3.28 \mu \mathrm{m}$ to measure benzene at concentrations down to $50 \mathrm{ppb}^{[232]}$.

OPOs also make use of mixing between a pump, signal and idler beam in nonlinear crystals. In this case however, the signal beam is an output and wavelength tuning is achieved by altering the refractive indices of the crystal at the different wavelengths concerned. Momentum conservation 
requires that $n_{p} \nu_{p}=n_{s} \nu_{s}+n_{i} \nu_{i}$, where $n_{p}, n_{s}$ and $n_{i}$ are the refractive indices at the pump, signal and idler wavelengths respectively, and likewise $\nu_{\mathrm{p}}, \nu_{\mathrm{s}}$ and $\nu_{\mathrm{i}}$ are their respective frequencies ${ }^{[233]}$. As the crystals concerned are usually birefringent, the refractive indices can be altered by tilting the crystal, or a change in temperature can provide a relative change in the refractive indices. Compared to DFG systems, OPOs can deliver potentially greater wavelength flexibility (DFGs require the signal beam to be provided by a separate, tunable source) but can be sensitive to mechanical or thermal instability.

Recent advances in OPO design have resulted in designs that are more robust and practical than in previous years, even including use in breadboard - transportable instruments ${ }^{[234]}$. Using an intracavity design, high conversion efficiencies can be obtained. For example a $3 \mathrm{~W}$ pump power can provide $300 \mathrm{~mW}$ and $150 \mathrm{~mW}$ of signal and idler power respectively, with an overall electrical power of $10 \mathrm{~W}$, and mode-hop-free tuning over hundreds of $\mathrm{GHz}$ is possible ${ }^{[235]}$. OPOs have enabled highly sensitive detection of hydrocarbons in the 3-4 $\mu \mathrm{m}$ region. By using a cavity-enhanced technique with an effective optical pathlength of around 3.5km, von Basum et al achieved an $\alpha_{\min }$ of $1.6 \times 10^{-10} \mathrm{~cm}^{-1} \mathrm{~Hz}^{-1 / 2}$ to give a limit of detection for ethane of $6 \mathrm{ppt} \mathrm{Hz} \mathrm{H}^{-1 / 2}[236]$.

\subsubsection{Associated light sources}

Although not strictly lasers, the following light sources offer similar advantages and employ pump lasers in their generation, so they are included here. First, we consider new developments in supercontinuum (SC) sources using various types of optical fibre. Emitted beams have a high spectral density and are spatially coherent with low divergence ${ }^{[237]}$, therefore SC sources can be combined with long pathlength gas cells including the cavity-enhanced schemes of section 7. Although the generation process is relatively complex, involving several steps, all-fibre implementation means that sources are relatively rugged ${ }^{[237]}$.

Typically, a beam from a pump laser is confined in a highly nonlinear fibre whose dispersion and other properties have been tailored to enhance nonlinear processes including self-phase modulation, four wave mixing and stimulated Raman scattering. Additional wavelengths are thus generated that merge to form a continuum. Supercontinua have been generated in standard telecoms fibre ${ }^{[238,239]}$ and optical fibre tapers ${ }^{[240]}$, however the use of femtosecond pulsed pumps with specially tailored PCF generally offers the highest conversion efficiency ${ }^{[240,241]}$. Through the use of chalcogenide fibre, SC spectra have been extended to mid IR wavelengths of up to $5 \mu \mathrm{m}^{[242]}$, as shown in Figure 43.

SC sources are often configured as non-dispersed broadband sources requiring a dispersive spectrometer to analyse and measure gas absorption. However, by employing a dispersion compensating module, the emission can be engineered to undergo rapid wavelength sweeps over the course of each pulse. Time-resolved spectra can then be recorded using a suitably fast detector / amplifier at repetition rates of up to around $1 \mathrm{MHz}^{[237]}$. Pulse-to-pulse variation is high, however by averaging over a large number of pulses and carefully measuring simultaneous reference spectra, good performance can be achieved. Near IR measurement of methane has been demonstrated over the $1.63-1.7 \mu \mathrm{m}$ range with an NEA of $4 \times 10^{-3}$ for a $1 \mathrm{~ms}$ averaging time $\mathrm{e}^{[237]}$. Similarly Sanders has made rapid scanned (every $20 \mathrm{~ns}$ ) measurements of water vapour, $\mathrm{CO}_{2}$, ethylene and ethanol at relatively high pressure (10 bar) in the range $1.35-1.55 \mu \mathrm{m}^{[243]}$. The equivalent spectroscopic linewidth of this source, limited by the scan rate and 300 fs pulse width, was $1 \mathrm{~cm}^{-1}(\sim 0.2 \mathrm{~nm}$ or $30 \mathrm{GHz})$, so fine detail in the spectra was not fully resolved. 


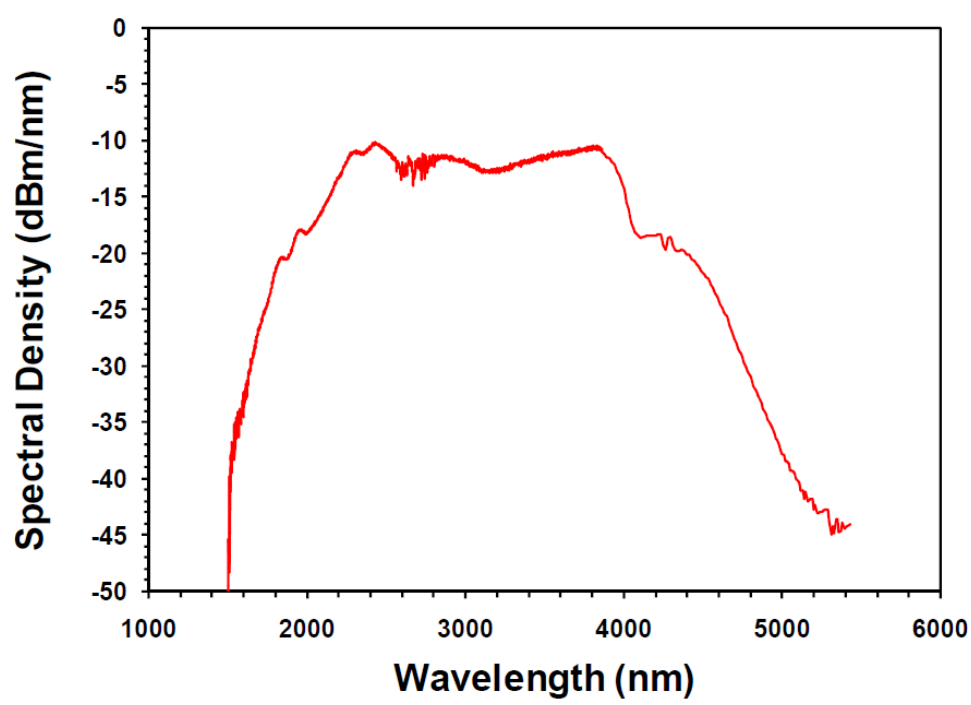

Figure 43. Spectrum of a supercontinuum generated in chalcogenide fibre, extending into the mid IR.

Taken from [242].

A final development is the use of frequency combs for gas spectroscopy. Originally developed for precision time and frequency metrology, frequency combs employ femtosecond (fs) pulsed lasers to generate a comb of precisely evenly spaced wavelength emission modes over a wide spectral range, with a spacing uniformity that can be better than a few parts in $10^{17}{ }^{[244]}$. Their use in high resolution spectroscopy has been recently reviewed by Adler et $a^{[245]}$. Typically the comb is mode-locked rather than being spectrally tuned according to the modulation schemes described in previous sections. The narrow spacing between modes of the comb $\left(250 \mathrm{MHz}\right.$ for Er doped optical fibre based sources ${ }^{[245]}$, corresponding to $0.002 \mathrm{~nm}$ or $0.01 \mathrm{~cm}^{-1}$ at $1.55 \mu \mathrm{m}$ ) actually makes them potential replacements for broadband sources used in spectrophotometry, albeit with high spectral brightness and a collimated beam that enables the use of longer pathlength cells.

Initially, frequency combs were based around mode-locked Ti: sapphire lasers, and reliable combs have also been built based on Er doped optical fibre in the telecommunications band around $1.5 \mathrm{\mu m}^{[245]}$. Nonlinear frequency conversion has enabled comb generation from the UV to the far IR, with the important mid IR region including DFG and OPO based generation ${ }^{[246]}$. Adler et al have used a high power OPO with tunable centre frequency, giving a comb width that varied from $0.3 \mu \mathrm{m}$ to $0.09 \mu \mathrm{m}$ over the wavelength region $2.9 \mu \mathrm{m}$ to $4.3 \mu \mathrm{m}$, respectively ${ }^{[247]}$. As illustrated in Figure 44 , the light was analysed with an FTIR spectrometer, which limits the spectral resolution that can be achieved with this technique. Adler et al achieved a noise limited $\alpha_{\text {min }}$ of $3.8 \times 10^{-8} \mathrm{~cm}^{-1} \mathrm{~Hz}^{-1 / 2}$ for each spectral element of their FTIR, which is some orders of magnitude better than the performance (where reported) of any of the developments of Table 7, for example. They measured absorption spectra of a range of analytes in the $2.9-4.6 \mu \mathrm{m}$ range, including nitrous oxide, formaldehyde, methane, ethane and isoprene. Compared with a conventional FTIR employing an incoherent source, measurements have higher spectral brightness and a better signal to noise ratio, which in turn enables shorter averaging times and faster FTIR scanning. 


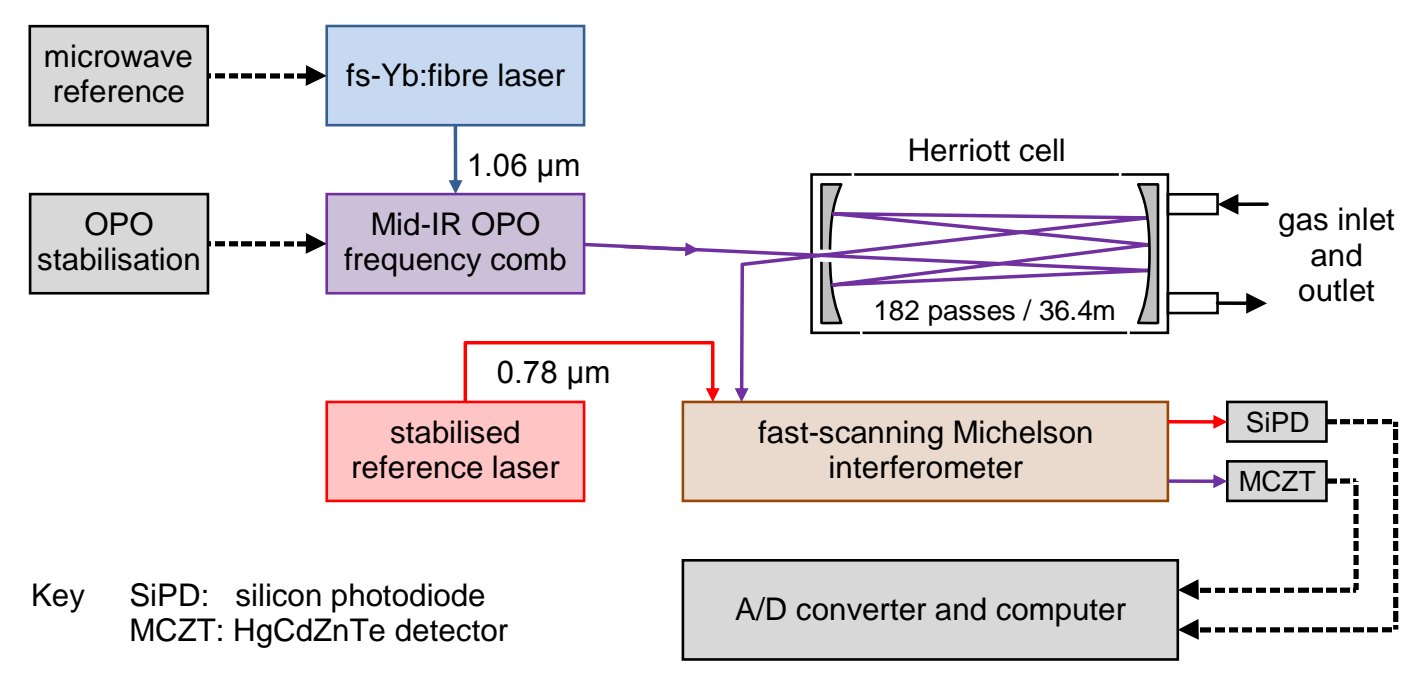

Figure 44. Use of a mid IR frequency comb as a source in FTIR spectroscopy. The collimated beam enables the use of a multipass Herriott cell. After [247].

Following a proposal by Schiller ${ }^{[248]}$, Coddington et al showed that by using two frequency combs with slightly different spacing, a continuously varying time delay was generated ${ }^{[249]}$, in the manner of the phase delay in the scanning Michelson interferometer of an FTIR spectrometer. The transmitted spectrum can be recovered by inverse Fourier transform of the time domain signal, hence the method being termed frequency comb Fourier transform spectroscopy (FC-FTS) ${ }^{[245]}$. Coddington et al demonstrated measurement of the absorption spectrum of HCN gas using a frequency comb centred on $1.545 \mu \mathrm{m}$, with an NEA of $5 \times 10^{-4}[250]$, and Mandon et al have measured the spectrum of acetylene over an $80 \mathrm{~nm}$ range centred on $1.5 \mu \mathrm{m}^{[251]}$. In the mid IR, Baumann et al have made measurements of the methane spectrum at $3.4 \mu \mathrm{m}$ with an NEA of $3 \times 10^{-4}{ }^{[252]}$, and Schliesser et al used a GaSe element to provide a DFG based mid IR dual comb spectrometer covering the 9-12 $\mu \mathrm{m}$ region ${ }^{[253]}$. They demonstrated detection of ammonia in a $10 \mathrm{~cm}$ pathlength cell as well as remote detection of the gas over a $22 \mathrm{~m}$ path. Therefore, these developments appear to combine the signal to noise and resolution advantages of TDLS with the wide spectral coverage of FTIR, including the important mid IR spectral region. Compared to an FTIR, as yet the spectral coverage is more limited and the systems are more complex, but the lack of moving parts is an important advantage.

\subsection{Standoff detection of backscattered light}

Lasers provide the opportunity for novel detection geometries including remote standoff detection of gases, typically based on tunable diode lasers operating over distances of $10 \mathrm{~m}$ or more ${ }^{[186,254,255]}$. These systems use a laser beam aimed through open space at a target, collecting the backscattered light and applying a spectroscopic signal demodulation. Applications include the detection of natural gas leaks from low-pressure distribution pipes, with a methane detector based on a tunable DFB laser emitting light at $1.65 \mu \mathrm{m}$. The optical pathlength is variable and unknown, therefore performance is often quoted in a similar fashion to that of open path gas detectors, using the pathlength - integrated unit of ppm.m. Iseki et al have modelled their system's reflectivity and noise performance, relating this to a minimum detectable gas concentration signals in ppm.m for different circumstances of target 
reflectivity and distance ${ }^{[254]}$. They demonstrated gas leak detection with leak rates as low as 0.01 litre $\mathrm{min}^{-1}$ and concentrations of around 100 ppm.m.

Systems of this type are typically limited by the level of optical backscatter from the surface, which can be as low as $5 \%$. For diffuse scattering from horizontal ground and a laser diode with power of the order of $10 \mathrm{~mW}$, the level of light received at the photodiode can be in the $\mathrm{nW}$ range ${ }^{[186]}$, despite the use of large (15 cm diameter) high NA lenses, therefore (unusually for TDLS) detector noise dominates. Improvements have been made using higher power sources, through optical amplification of standard DFB laser output ${ }^{[256]}$, however for some applications this would compromise eye safety in public areas.

Laser pointers preferentially sample the gas in a thin layer close to the ground in a highly localised area downwind of the leak source ${ }^{[257]}$. For this reason, the correspondence between the standoff measurement and that of a point sampling instrument is surprisingly good, as Figure 45 shows.
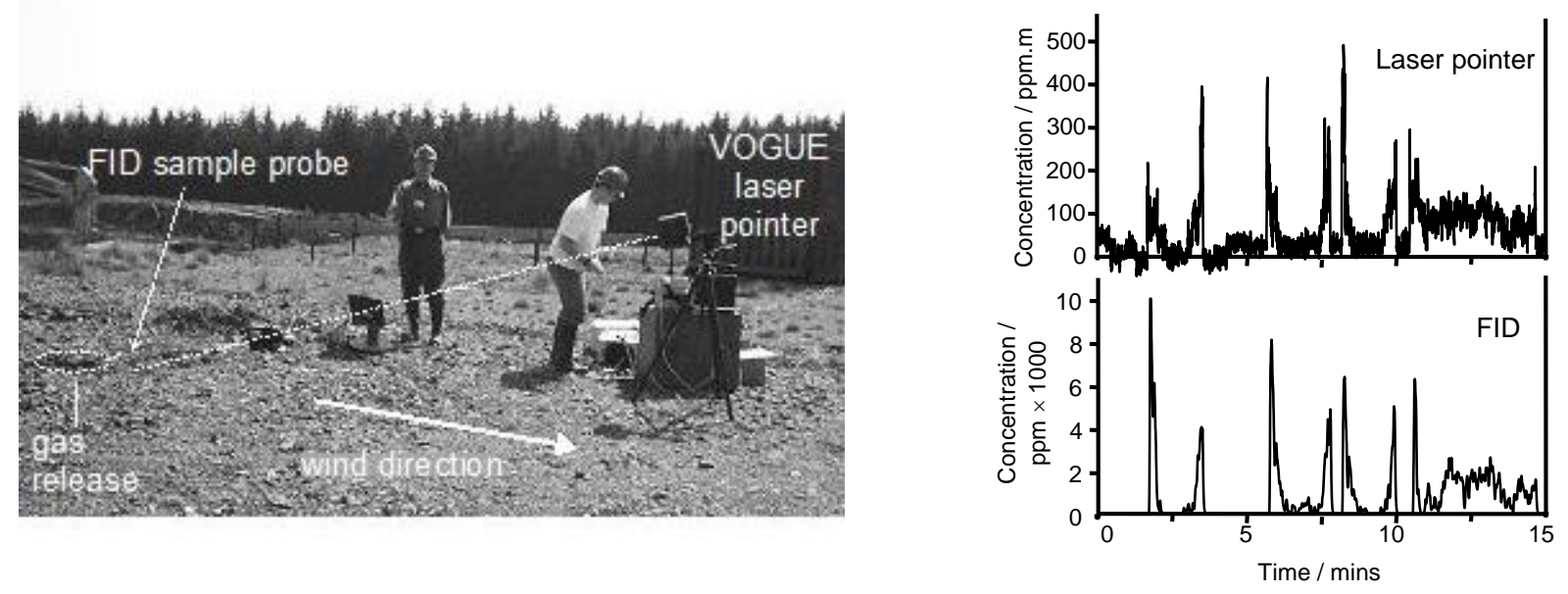

Figure 45. Correspondence between field measurements from a prototype laser pointer and flame ionisation detector (FID) targeted at the same sampling location, taken during a release of natural gas at 10 litre $\mathrm{min}^{-1}$. Taken from [257].

By scanning the laser pointer in a raster fashion, it is possible to construct a low resolution 2-D image of the gas plume ${ }^{[258]}$ (the $10 \times 10$ pixel resolution and 1 frame/s refresh rate being limited by the very low light intensities at the detector). In comparison with passive gas imaging using the NDIR principle or gas correlation cells, the main advantage is that the technique provides a reliable zero measurement in the absence of a gas leak. Higher power lasers can provide greater resolution and / or refresh rates, and the use of the mid IR again improves detection limits. For example, Stothard et al used a $55 \mathrm{~mW}$ OPO source at $3.3 \mu \mathrm{m}$ to record images of methane leaks with $100 \times 150$ pixels and refresh rates of 3 frames/s, with a limit of detection of $30 \mathrm{ppm} \cdot \mathrm{m}^{[259]}$. 


\subsection{Light scattering in TDLS}

Recent research has considered the use of diffusely transmitting or reflecting optics as alternatives to windows or mirrors ${ }^{[260]}$. The advantages are very simple alignment and improved stability to temperature changes. In reflective cells, the optical configuration is similar to that found in the standoff laser pointers of the previous section, albeit with a fixed, known pathlength. Chen et al have used a diffuse reflector manufactured from anodised aluminium in order to improve sensor robustness in a challenging industrial environment (the exhaust of a combustion furnace), with background fluctuations at the level of $\Delta \mathrm{I} / \mathrm{I} \sim 10^{-5}{ }^{[188]}$. A group at Lund University have used TDLS to explore gas concentrations held within porous and scattering media including pharmaceutical samples ${ }^{[261]}$ and biological tissue ${ }^{[262]}$. Their work with oxygen confined in nanoporous media ( $y$-alumina) has shown that surprisingly long optical pathlengths can be achieved, and evidence for line broadening effects beyond levels expected for gases at atmospheric pressure ${ }^{[263]}$.

When such materials are used in high resolution spectroscopy, the well-known, structured interference fringes of conventional gas cell configurations are disrupted, interference effects still dominate. These are random interference, namely laser speckle ${ }^{[260]}$, interferometric speckle ${ }^{[264]}$ and self-mixing or feedback interference effects ${ }^{[194]}$. Although the latter exist in many conventional TDLS schemes, they can be particularly troublesome for diffusely reflecting materials. Similar interference effects are also observed when using integrating spheres as gas cells, because the inner surface of these devices is designed to closely match a perfect Lambertian (diffuse) scatterer ${ }^{[35]}$. Many of these undesirable effects can be reduced by vibrating or rotating the scattering target ${ }^{[263,260]}$.

\section{Cavity enhanced techniques}

Significant recent activity in this area merits its inclusion as a separate section. Furthermore, cavityenhanced cells have been used with all three spectral readout or demodulation techniques described above (non-dispersive, spectrophotometry and TDLS), which have much in common when applied to cavity - enhanced systems. General reviews of cavity-enhanced techniques and their relative performance have been produced by Paldus and Kachanov ${ }^{[265]}$, and Foltynowicz et al ${ }^{[266]}$.

In each of the techniques described here, extremely long pathlength cells are constructed using high reflectivity $(R>99.99 \%)$ mirrors, which can be achieved using dielectric coatings. Resonant cavities are formed in the manner of a high finesse Fabry-Perot etalon. The finesse of the cavity, $F$, is given by ${ }^{[245]}$ :

$$
F=\frac{\pi \sqrt{R}}{(1-R)} \approx \frac{\pi}{(1-R)} \text { for high } \mathrm{R}
$$

And the pathlength enhancement (ratio of average pathlength $L_{\text {eff }}$ to physical cell length $L$ ) is equal to $\beta F / \pi$, where $\beta$ is a factor that depends on the coupling scheme used, such that $1 \leq \beta \leq 2^{[245]}$. The equivalent pathlength of the cell, $L_{\text {eff, }}$, is then given by

$$
L_{\text {eff }}=\frac{\beta L}{(1-R)} \quad \text { for high } \mathrm{R}
$$


Enhancements can be up to $10^{5}$ using mirrors with $R$ of up to $99.999 \%{ }^{[266]}$, significantly better than for standard multipass cells (see Table 3 ) where the best enhancements are of the order of $10^{2}-10^{3}$. Mirrors of this quality may also be referred to by their optical loss in ppm. One issue that this raises is the use of dichroic coatings to achieve such high mirror reflectivities. The coatings restrict the wavelength range of operation, and the small changes in $R$ over that range result in a varying optical pathlength as a function of wavelength. Figure 46 illustrates the variation in $R$ and $\mathrm{F}$ with wavelength for a typical high finesse cavity in the near IR. Finally, to maintain cell performance it is vital to ensure that mirrors remain clear of dust or condensation during use, either of which would contribute to optical losses, reducing $R$.

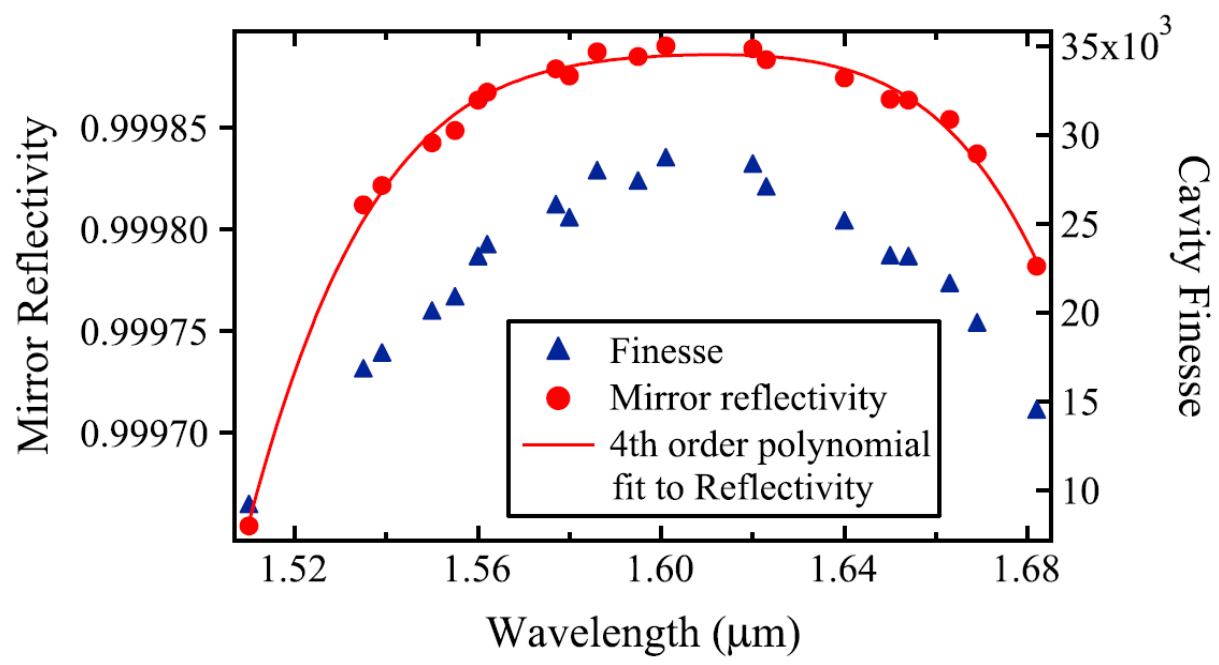

Figure 46. Variation in reflectivity $R$ and finesse $F$ for a typical high finesse cavity in the near IR, illustrating the effect of using dichroic mirrors on the wavelength range of operation. Taken from [246].

The free spectral range of the cavity is of the order of hundreds of $\mathrm{MHz}$ for cavities whose physical length $L$ is tens of $\mathrm{cm}$ long $(\mathrm{FSR}=\mathrm{c} / 2 L)^{[266]}$. For tunable lasers with linewidths of the order of tens of $\mathrm{MHz}$, it can therefore be a challenge to couple to the cavity efficiently because of the narrow linewidth of cavity modes. There are two main choices; (i) to couple to a single cavity mode, adjusting the cavity length to locking it to the laser emission, which increases complexity, or (ii) to average over a large number of modes, which reduces the mean coupling efficiency. The different techniques described below adopt different approaches to address these issues.

\subsection{Cavity ringdown spectroscopy}

Cavity ringdown spectroscopy (CRDS) was pioneered by O'Keefe and Deacon ${ }^{[267]}$, building on a technique previously used to measure mirror reflectivity. Reviews of different instrumental schemes have been completed by Berden et al (2000) ${ }^{[268]}$, Brown $(2003)^{[269]}$ and more recently for optical fibre based cavities by Waechter et al (2010) ${ }^{[47]}$. There is also a recently published book on the subject, edited by Berden and Engeln ${ }^{[270]}$.

Light coupled into a high finesse cavity builds up and decays exponentially as the source is turned on and off. Absorption measurements are made via the exponential decay time; any loss in the cavity results in a reduction of the decay time, and as long as overall losses are small, decay times can be 
measured using high sensitivity detectors operating with $\mathrm{kHz}$ bandwidths. Sources of loss in the cavity include mirrors with less than 100\% reflectivity, scattering and imperfections in alignment (all of which have broadband effects) as well as spectral absorption by the target species, which may be distinguishable by a spectral baseline or reference measurement. The exponential decay envelope of the measured signal takes the general form

$$
I=I_{0} \exp \left(-\frac{t}{\tau}-\alpha c t\right)
$$

Where $\tau$ is the characteristic decay time in the absence of absorption. The following source modulation techniques have been used in CRDS ${ }^{[47]}$ (see Figure 47):

(a) A CW source may be switched off rapidly (directly or using an external modulator), after which the detector observes smooth exponential decay. Alternatively, the source and cavity modes may be rapidly swept with respect to one another. The term cavity leak-out spectroscopy (CALOS) has also been used ${ }^{[271]}$.

(b) A single input pulse results in a series of pulses at the detector within an exponentially decaying envelope. The pulses may be filtered out by suitable choice of detection bandwidth to leave a smooth envelope.

(c) For a rapidly modulated CW source, a phase shift is introduced at the detector, equal to $\tau$. Phase shift cavity ringdown spectroscopy (PS-CRDS) employs simple analogue detection electronics, avoiding the need to digitise ringdown traces and fit exponential decay curves

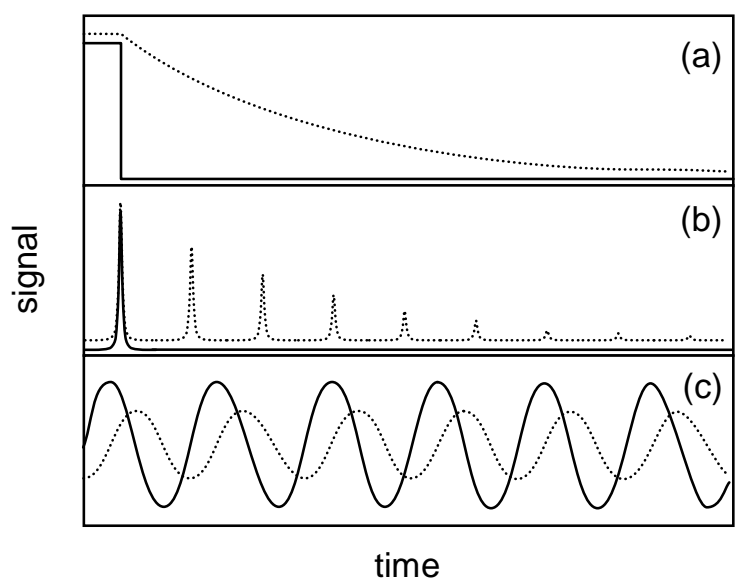

Figure 47. Different forms of CRDS, showing detector signals (dotted lines) for the following forms of input (solid lines): (a) CW source switched off rapidly; (b) single pulse input; (c) rapidly modulated CW source used in phase shift CRDS. Modified from [47].

O'Keefe and Deacon discussed the important characteristics of pulsed CRDS ${ }^{[267]}$. By using laser pulses shorter than the cavity round trip time, the problem of coupling to specific modes within the cavity is avoided and the emission wavelength may be scanned over an absorption feature. The method has the advantage of being relatively insensitive to power fluctuations, since the ringdown time is determined by measuring the relative intensity decay. This makes the technique particularly suited to sources that must be operated in pulsed mode, as pulse-to-pulse intensity variation can otherwise 
be a significant source of noise. A disadvantage is the need to fit the recorded trace to an exponential decay; multiple exponential decay terms can cause problems in fitting ${ }^{[272]}$.

As with other absorption measurements, a zero reference can be recorded without an absorber in the cell, more than one signal can be recorded at different (non-absorbing) wavelengths, or many signals recorded as a function of wavelength, the zero determined using a baseline correction. $\alpha_{\min }$ values of $10^{-10} \mathrm{~cm}^{-1} \mathrm{~Hz}^{-1 / 2}$ are achievable, often limited by system drifts between one ringdown event and the next ${ }^{[266]}$ and noise resulting from excitation of multiple cavity modes by pulsed sources with relatively broad linewidths ${ }^{[273]}$. This is an improvement over standard TDLS, largely because equivalent cell pathlengths can be of the order of $10 \mathrm{~km}$. Use of confocal cavities enables relatively simple construction (see Figure 48). The technique has been used in an industrial application subject to vibration (measurement of emissions on carbon sequestration sites) ${ }^{[274]}$ and has formed the basis of a commercial instrument ${ }^{[363]}$.

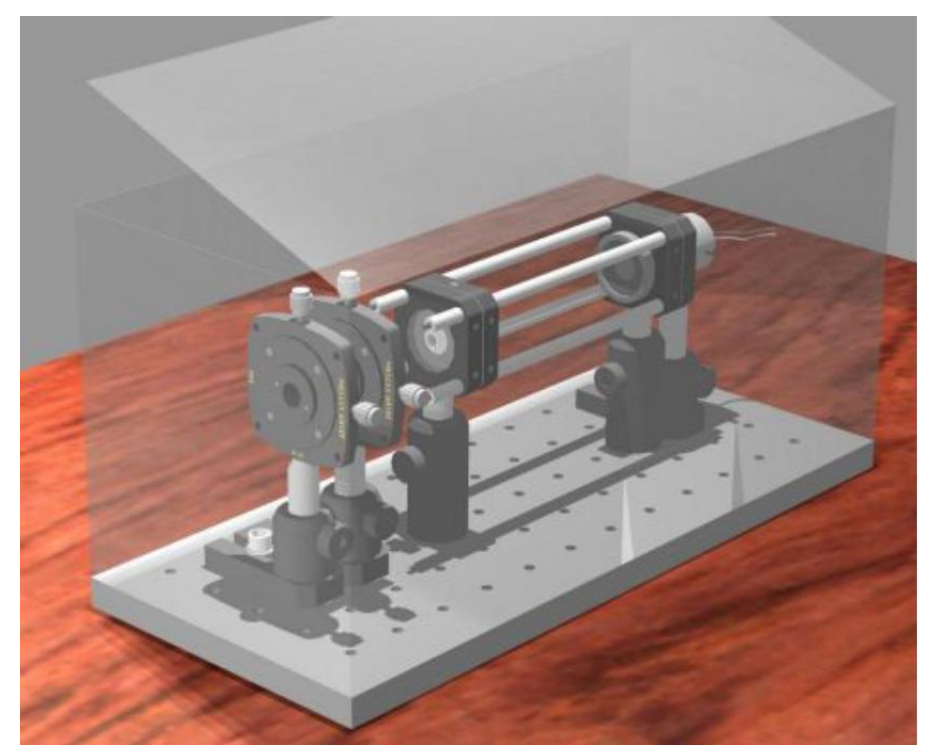

Figure 48. Cavity ringdown absorption cell using standard optomechanical components to give a physical length of $15 \mathrm{~cm}$ and equivalent pathlength of $12 \mathrm{~km}$, used to detect acetone with a $266 \mathrm{~nm}$ pulsed laser. Taken from [275].

He and Orr developed a CW scheme involving a rapidly swept cavity length using a piezoelectric actuator, measuring build-up and ringdown times as cavity modes moved in and out of correspondence with laser emission ${ }^{[276]}$. Use of a heterodyne receiver to detect light reflected from the cavity also enabled the separation of the ringdown cell from the transceiver optics such that the two can be linked via optical fibre. For the basic cell, an $\alpha_{\min }$ of $5 \times 10^{-10} \mathrm{~cm}^{-1} \mathrm{~Hz}^{-1 / 2}$ was achieved with a relatively simple configuration. $\alpha_{\min }$ values of $10^{-9} \mathrm{~cm}^{-1}$ are typical for this technique ${ }^{[265]}$, limited by variations in the level of light injected into the cavity from one event to the next, as a result of coupling to variable cavity modes ${ }^{[265]}$. Huang and Lehman have shown that the fundamental sensitivity limit for the swept-cavity technique is worse than for techniques using a modulated CW source, and its success relies on the use of sources such as external cavity diode lasers that have low phase noise $e^{[277]}$. 
By using CW tunable lasers with narrow linewidths and locking a single cavity mode to the laser emission, the statistical variations of different modes are avoided ${ }^{[265]}$. An $\alpha_{\min }$ as low as $8.8 \times 10^{-12} \mathrm{~cm}^{-1} \mathrm{~Hz}^{-1 / 2}$ has been achieved at low operating pressures (1-3 Torr) ${ }^{[273]}$. Using an adaptation of the now well-established Pound-Drever-Hall technique ${ }^{[278]}$, two orthogonal polarisation states were employed, one used for mode locking in a servo loop and the other carrying the ringdown signal. This approach ensured that mode locking was achieved under the same conditions as ringdown measurement, being matched in both wavelength and beam position within the cavity during a ringdown event. A variation on this approach had one beam acting as a local oscillator in a heterodyne detection scheme and the other, with a slight frequency shift, carrying the ringdown signal ${ }^{[410]}$. The local oscillator beam was used to lock one TEM $_{00}$ cavity mode to the laser emission and the second coupled to another $\mathrm{TEM}_{00}$ mode and used for ringdown measurements. An NEA of $2 \times 10^{-9} \mathrm{~cm}^{-1}$ was achieved for a measurement integration time of around $2 \mathrm{~ms}$.

Finally, laser self-locking has been achieved by allowing a proportion of the light reflected from the cavity to feed back to the laser. A technique known as optical feedback CEAS (OF-CEAS) employs a V-shaped cavity as shown in Figure 49. With this method, Romanini et al were able to solve the problem of direct feedback from the cavity input mirror and feed back only wavelengths corresponding to cavity modes ${ }^{[279]}$. The distance from laser to cavity was carefully controlled such that it remained in phase with the laser emission, resulting in a line narrowing effect, which could be beneficial in high resolution studies. During a laser wavelength scan the laser jumps or mode-hops from one cavity mode to the next, each time giving a measurement perfectly aligned with the maximum transmission peak of that mode, enabling higher precision spectroscopic data. Morville et al demonstrated $\alpha_{\text {min }}$ better than $10^{-9} \mathrm{~cm}^{-1} \mathrm{~Hz}^{-1 / 2}$ with this system, and also noted its relative simplicity and robustness compared to their standard CW CRDS set-up ${ }^{[280]}$.

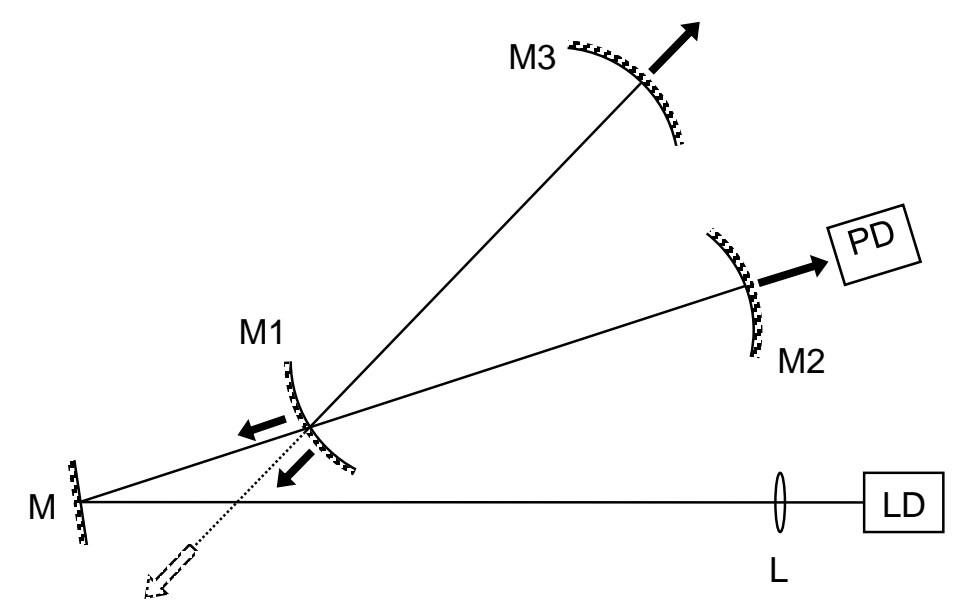

Key L: lens, LD: laser diode, M: mirror, PD: photodiode

Figure 49. V-shaped cavity used for optical feedback CRDS and CEAS (OF-CRDS and OF-CEAS).

The phase of the feedback is controlled using a PZT actuated mirror. After [280].

Engeln et al reported the first use of phase shift cavity ringdown spectroscopy (PS-CRDS) for gas detection, applied to a weak $\mathrm{O}_{2}$ absorption line at $635 \mathrm{~nm}^{[281]}$. Using a $45 \mathrm{~cm}$ cell with $R \sim 99.85 \%$ mirrors gave a ringdown time of approximately $600 \mathrm{~ns}$, detected with a lock-in amplifier as a phase 
shift of a $2 \mathrm{MHz}$ intensity modulation applied to a CW laser beam. Absorption measurements were made by normalising the out of phase component of the modulation by the in phase component, thus making the measurement independent of intensity variation. Further advantages of this technique are that the duty cycle is improved (from a typical $0.01 \%$ to an effective $50 \%{ }^{[281]}$ ), giving an increased signal : noise ratio, and no stabilisation of the cavity is required. Van Helden et al have shown that by correcting for the effects of amplified spontaneous emission (ASE) from the laser source, accurate quantitative measurements can be made, and demonstrated an $\alpha_{\min }$ of $2 \times 10^{-8} \mathrm{~cm}^{-1}$ around $800 \mathrm{~nm}$ with an integration period of $0.5 \mathrm{~s}^{[282]}$. A disadvantage of PS-CRDS is that the light couples efficiently into cavity modes only $5-10 \%$ of the time ${ }^{[283]}$, depending on the finesse of the cavity.

Finally, Nikolaev et al have reported a different scheme in which light entered the cavity at a slight angle off-axis, and was subsequently detected by a series of spatially separated detectors, each corresponding to a different mean pathlength ${ }^{[284]}$. Thus, the ringdown time could be determined by the difference in intensity measured by each of the photodiodes, rather than in the time domain, avoiding the need to turn the light source on and off and also avoiding the issue of mode coupling to the cavity. An advantage was the abilty to record spectral data more rapidly (one data point every $5 \mu \mathrm{s}$ ). An $\alpha_{\min }$ of $8 \times 10^{-7} \mathrm{~cm}^{-1}$ was estimated for averages over 100 neighbouring spectral points, corresponding to an integration period of $0.5 \mathrm{~ms}$.

CRDS has been used with broadband CW sources as well as with lasers. The broad emission of the source covers a large number of cavity modes and the measurement essentially averages over these, but overall coupling efficiency is reduced as a result. Ball et $a^{[285]}$ used broadband radiation from a $\mathrm{Nd}$ :YAG pumped pulsed dye laser at $662 \pm 2 \mathrm{~nm}$ to detect the $\mathrm{NO}_{3}$ radical in a cell with $95 \mathrm{~cm}$ base length and mirrors with $R>99.995 \%$ over the wavelength range. Light from the cavity was directed to an imaging spectrometer where, by clocking the CCD array, ringdown times across the wavelength range could be obtained. An $\alpha_{\min }$ of $4 \times 10^{-8} \mathrm{~cm}^{-1}$ was estimated. The principle has been extended to use of a visible $L E D^{[286]}$, with a photomultiplier tube used to detect ringdown events with sufficient sensitivity to compensate for the low optical throughput. Hamers et al have developed PS-CRDS for use with a CW xenon arc lamp in an FTIR spectrometer, measuring Fourier transformed in-phase and out-of-phase components of the signal ${ }^{[287]}$. With this technique they were able to demonstrate detection of $\mathrm{O}_{2}$ around $764 \mathrm{~nm}$, with spectral coverage limited in principle by the bandwidth of their high reflectivity dichroic mirrors ( $R>0.999$ over the range $700-900 \mathrm{~nm}$ ).

Problems can arise with low overall coupling efficiencies in very high finesse cavities, especially for many broadband sources with relatively low spectral densities. However, Petermann and Fischer ${ }^{[288]}$ have shown that an acousto-optic modulator (AOM) within the cavity can be used to divert light into and out of the cavity, allowing efficient coupling during the build-up phase and active "dumping" of the light onto a photodetector at specified points in the ringdown phase. Losses due to the AOM were limited to 0.002 per round trip, so that the cavity finesse was not compromised. The resulting $\alpha_{\min }$ was estimated at $1.8 \times 10^{-8} \mathrm{~cm}^{-1}$ for a single spectral channel containing $\mu \mathrm{W}$ of power, with a total measurement time (for a full spectrum) of $25 \mathrm{~s}$.

Although CRDS has achieved remarkable equivalent pathlengths and detection limits, it relies on the use of fast detection electronics to quantify small differences between ringdown times that may be in the ms- $\mu \mathrm{s}$ regime, and fit its form to an exponential decay curve, sometimes with more than one exponential term. This requires digitisers that are both fast (in the $100 \mathrm{MHz}$ regime) and high precision (14-16 bit). Use of the Levenberg - Marquardt fitting algorithm can be computationally intensive, 
limiting the speed of the technique ${ }^{[265]}$. A number of analogue demodulation techniques have therefore been proposed. Spence et al demonstrated that a logarithmic amplifier can be used to convert an exponentially decaying signal to a square wave ${ }^{[273]}$, but the electronics required were prohibitively complex. Faster fitting algorithms have also been proposed to give $10 \mathrm{kHz}$ measurement rates ${ }^{[289]}$, as well as frequency domain analysis via fast Fourier Transforms (FFT) of pulsed ringdown decays ${ }^{[290]}$ and square wave modulated CW light ${ }^{[291]}$.

\subsection{Cavity-enhanced absorption spectroscopy}

In principle, what distinguishes cavity ringdown spectroscopy from cavity-enhanced absorption spectroscopy (CEAS) is that CRDS measurements are made in the time domain, with the measured absorption being a direct function of ringdown times or phase shifts. In CEAS, the measured intensity of light leaking out from the cavity is integrated over time periods much longer than the ringdown time, enabling the use of simpler electronics while still retaining the long pathlengths of high-finesse cavities. The detection bandwidth required for CEAS can be in the region 10-200 kHz compared with $>1 \mathrm{MHz}$ for CRDS, depending on the finesse of the cavity ${ }^{[292]}$. The technique was first demonstrated in two almost simultaneous papers: by O'Keefe ${ }^{[293]}$ (referred to as integrating cavity output spectroscopy, ICOS), and Engeln et al ${ }^{[294]}$ (referred to as cavity enhanced absorption spectroscopy, CEAS). Many practitioners now use the term ICOS for the techniques involving integration over a large number of cavity modes by dithering either the cavity length and / or the laser wavelength ${ }^{[295]}$, with CEAS reserved for alternative variations (see below), and this is the convention adopted here.

A large number of closely spaced cavity modes is excited; mode fluctuations dictate that the larger the number that can be averaged, the better the noise characteristics. To increase the degree of averaging, the cavity modes may be dithered by a PZT actuator on one of the cavity mirrors on a timescale faster than the integration time for each measurement point. To avoid undesirable saturation effects associated with the build-up of light in the cavity (as in CRDS), the cavity must also be dithered on a timescale faster than the ringdown / build-up time ${ }^{[295]}$. Alternatively the laser emission wavelength can be dithered, to capture more cavity modes, with an amplitude that still permits resolution of the gas line. Limitations to ICOS include the statistical residual uncertainty caused by intentional coupling to a finite number of cavity modes, and the low mean optical throughout.

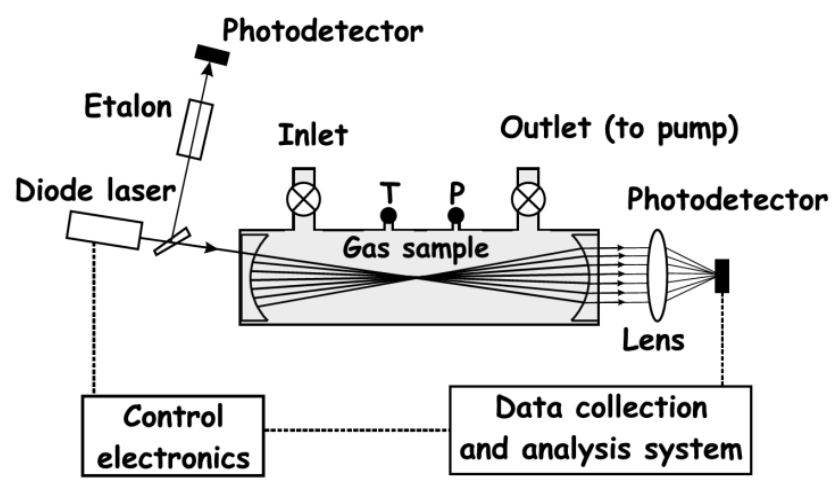

Figure 50. Off-axis integrated cavity output spectroscopy (OA-ICOS) as implemented by Baer et al, taken from [296]. The etalon was used for relative wavelength measurements during a scan by the laser diode. 
A development is the use of off-axis ICOS (OA-ICOS), shown in Figure 50. The method was introduced by Paul et al ${ }^{[297]}$ and their paper clearly explains the basic principles. The off-axis geometry increases the round-trip time of the cell by arranging a re-entrant condition after a large number of passes in the manner of a Herriott cell; both conventional and astigmatic variants are possible in principle. This in turn creates a more densely spaced series of cavity modes with a narrower FSR, as illustrated in Figure 51.

For very long re-entrant conditions, the mode spacing becomes narrower than the mode width, the latter governed by slight mechanical imperfections of the cavity. The cavity transfer function then no longer contains discrete Fabry-Perot modes, but ideally approaches an average transmission that is constant as a function of wavelength, albeit a low transmission $\left(T \sim 0.5 \%\right.$ for Paul et $a{ }^{[297]}$ ). Thus, intensity fluctuations due to mode instabilities are minimised. There are a number of practical benefits compared with standard ICOS including simpler alignment, reduced system complexity and reduced feedback / interference effects because of the off-axis injection of light into the cavity ${ }^{[297]}$. The result is a highly sensitive method that is capable of field deployment, and can be used with both direct spectral scanning of the gas line ${ }^{[296]}$, and $2 f \mathrm{WMS}^{[298,299]}$, with NEAs in the range $10^{-6}-10^{-7}$, often at reduced sample pressure. The reader is referred to a detailed discussion of the design considerations and compromises required in OA-ICOS written by Moyer et $\left.a\right|^{[300]}$, with specific reference to the Harvard system used for airborne measurement of water isotopologues.

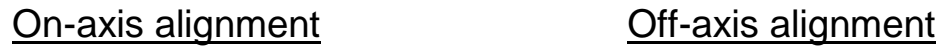

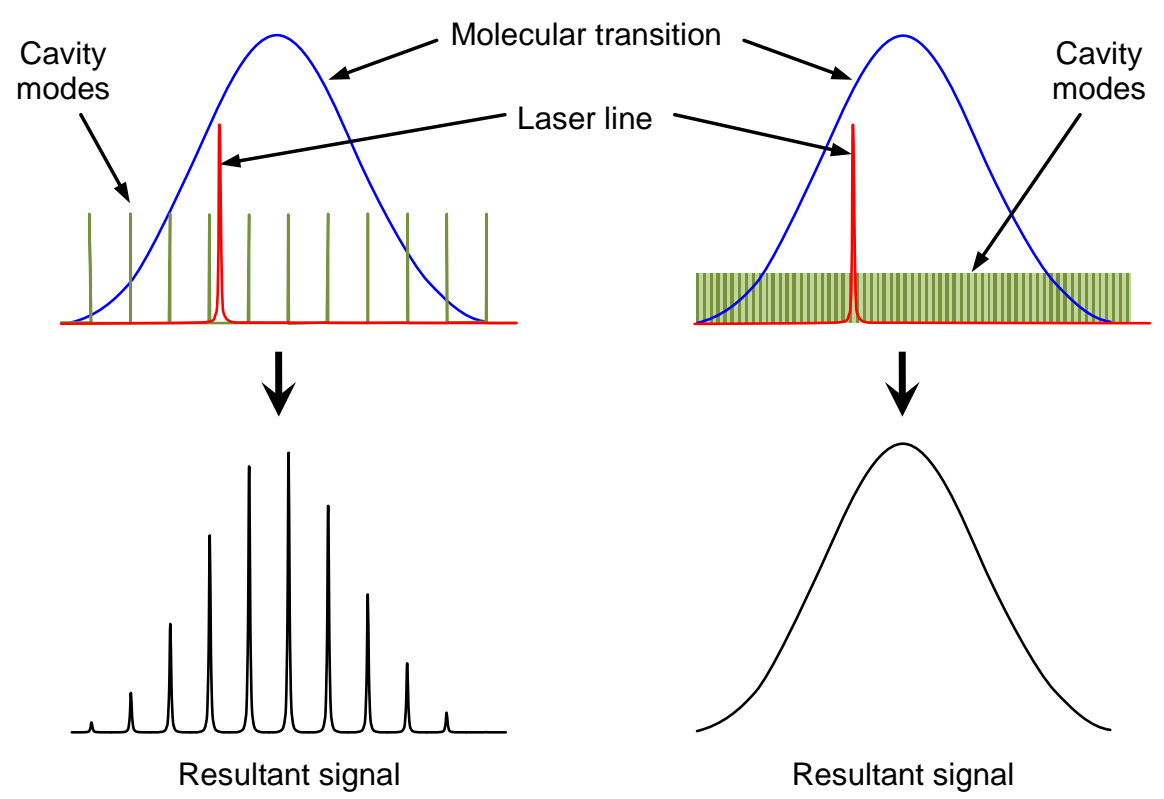

Figure 51. Comparison of on-axis and off-axis ICOS, showing the cavity mode structure superimposed on the gas absorption lineshape. In the off-axis case the cavity mode structure collapses to a continuum or quasi-continuum in which the FSR is much narrower than the laser linewidth and cannot be resolved. After [292].

Engel et al ${ }^{[292]}$ have demonstrated an $\alpha_{\min }$ of $1.9 \times 10^{-12} \mathrm{~cm}^{-1} \mathrm{~Hz}^{-1 / 2}$ for direct spectroscopy via OAICOS, at reduced pressure (200 Torr) and using a $110 \mathrm{~cm}$ base length cavity with an equivalent 
pathlength of approximately $2.7 \mathrm{~km}$. The system was limited by unwanted transient effects caused by coupling to spurious cavity modes, taking the appearance of white noise at the detector. Other limitations to OA-ICOS include, again, the low optical throughput. Dyroff has pointed out ${ }^{[301]}$ that for a given cell base length and source, there is an optimum mirror reflectivity / pathlength that depends on the source power and level of detector noise; longer pathlengths may not always be better in this respect as they require higher reflectivities that reduce the received power at the detector.

A practical issue with such techniques is the need for calibration; the equivalent pathlength of the cell is a sensitive function of the mirror reflectivity (see equation (10)). Even if the reflectivity of the mirror coatings can be known with sufficient precision at the wavelength of operation, in practice cavity losses can be introduced by small changes in geometry during alignment, scattering from imperfections in the mirror surfaces, or surface degradation in use. Maintaining a stable pathlength under field conditions is an engineering challenge, requiring thermo-mechanical stability, isolation from dust in sampled air and temperature management to avoid condensation of water.

Fortunately, self-calibrating instruments have been developed that use time domain information to provide knowledge of the cell pathlength. A team at Los Gatos Research Inc developed an instrument for field use employing OA-ICOS ${ }^{[296]}$, for which the cavity modes approached a continuum. After scanning the laser wavelength through the gas line using direct spectroscopy, the laser was switched off rapidly and a ringdown measurement made at a non-absorbing wavelength. By measuring $\tau$ in equation (11) (equal to $C \cdot L_{\text {eff }}$ for a non-absorbing cavity), the value of $L_{\text {eff }}$ can be determined for every scanned measurement at a wavelength adjacent to the measurement wavelength (close enough to have effectively the same mirror reflectivity and therefore the same pathlength). This instrument has been in continuous development and used in a number of field campaigns ${ }^{[302,303,304]}$.

Also making use of time domain information, phase shift measurements have been used to calibrate the effective pathlength or ringdown time ${ }^{[305]}$. When the source intensity is modulated at a frequency approaching $1 / \tau$ (where $\tau$ is the ringdown time), a phase delay is observed between the input and measured modulations, measurable using two channels of a lock-in amplifier. As mentioned in the previous section, the phase delay itself has also been used to determine the absorption within the cavity; such techniques are equally well described as phase shift cavity ringdown or phase shift cavity enhanced spectroscopy (PS-CEAS). Kasyutich et al have employed the phase shift method in OAICOS and shown that it is possible to recover gas concentrations with high accuracy as long as a significant potential source of error, the effect of ASE of the laser source, is accounted for ${ }^{[283]}$.

Broadband CEAS (BB-CEAS) has been developed in the UV, visible and near IR using both incoherent and partially coherent sources, namely a Xe discharge lamp ${ }^{[306,307]}$, visible LEDs ${ }^{[308]}$, UV LEDs $^{[309]}$, a near IR superluminescent LED (SLED) ${ }^{[310]}$ and a supercontinuum source emitting from 630 to $720 \mathrm{~nm}^{[311]}$. In each case, the output was directed to a high sensitivity spectrograph or FTIR spectrometer and the incoherent or short coherence length of the sources meant that there was no need to either lock or dither the cavity. For $\mathrm{NO}_{2}$ measurement, a compact and robust instrument has been made using optical fibre to couple light from an LED emitting in the 441 - $462 \mathrm{~nm}$ range, though a cell with base length $1.5 \mathrm{~m}$ and mirror reflectivity $99.976 \%{ }^{[312]}$. Its limit of detection for $\mathrm{NO}_{2}$ was between 100 and $240 \mathrm{ppt}$, corresponding to an $\alpha_{\min }$ of between $3 \times 10^{-9} \mathrm{~cm}^{-1}$ and $8 \times 10^{-9} \mathrm{~cm}^{-1}$.

Obtaining a good calibration of optical pathlength can be an issue for broadband CEAS, as noted by

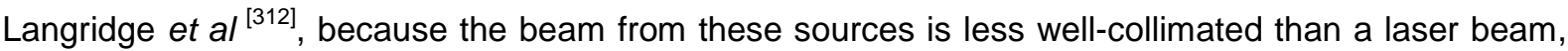
resulting in geometric light loss from the cavity and excitation of cavity modes with higher losses. Thus 
the effective pathlength must be determined using the same beam geometry as that used for measurement and not, for example, a better collimated laser beam at the same wavelength. Laurila et al solved this problem for their supercontinuum source by using an acousto-optic tunable filter (AOTF) to simultaneously provide narrowband light filtration and scanning enabling pathlength calibration by measurement of phase shifts in PS-CRDS, without affecting the alignment of the system in normal operation $^{[313]}$. A further issue for UV-visible spectroscopic techniques is the need to deconvolve potentially overlapping absorption features together with spectral losses caused by light scattering from aerosols over the long effective pathlengths used. Fortunately there is a wealth of such techniques used in DOAS (see section 5), where such long pathlengths are the norm.

Broadband $\mathrm{NO}_{2}$ measurement has been further developed by Kebabian et al ${ }^{[314]}$ using PS-CEAS in a method they refer to as cavity attenuated phase shift spectroscopy (CAPS). Here, the distinction between PS-CRDS and PS-CEAS breaks down - both involve measurement of phase shifts (time domain data) integrated over a measurement period. However, the techniques described as cavityenhanced often use an off-axis configuration, or broadband sources, in each case measuring over an integrated quasi continuum of cavity modes. In the work of Kebabian et al, the need for fast modulation and measurement of signal phases meant that spectroscopic measurements were not possible. Instead, a non-dispersive technique was used with a bandpass filter centred at $430 \mathrm{~nm}$. A limit of detection of $0.3 \mathrm{ppb}$ was demonstrated for an integration time of $600 \mathrm{~s}$, corresponding to an $\alpha_{\text {min }}$ of $10^{-8} \mathrm{~cm}^{-1} \mathrm{~Hz}^{-1 / 2}$. Shifting the measurement wavelength to $455 \mathrm{~nm}$ avoided spectral interference from neighbouring absorption bands of glyoxal and methyl glyoxal, however a potential interference with high levels of ozone remained ${ }^{[315]}$. Kasyutich et al reduced the likelihood of cross-species interference by using a narrowband $404 \mathrm{~nm}$ diode laser in an OA-CEAS configuration also involving phase shift measurements to give automatic self-calibration of the effective cavity pathlength ${ }^{[316]}$. Demonstrated performance was similar, with a detection limit for $\mathrm{NO}_{2}$ of $0.24 \mathrm{ppb}$. Because of the low level of spectral interference anticipated in urban air, such methods have the potential to replace the widespread use of chemiluminescent $\mathrm{NO}_{2}$ detectors for environmental research and air quality monitoring.

Various techniques have been used to improve mode-locked laser based CEAS, along similar lines to those already discussed for CRDS. By locking the laser emission to a single cavity mode, coupling efficiency improves dramatically and there can be a reduction in noise associated with laser instabilities. The Pound-Drever-Hall technique ${ }^{[278]}$ has been used, which adds experimental complexity and typically allows locking for several minutes ${ }^{[317]}$. Fortunately, a simpler and more stable self-locking variant of ICOS has been developed known as optical feedback cavity-enhanced spectroscopy, OFCEAS, with the same V-shaped cavity as that used in OF-CRDS (see Figure 49). With this, Morville et al have achieved an $\alpha_{\min }$ in their baseline of $5 \times 10^{-10} \mathrm{~cm}^{-1}$ for scans averaged over $1 \mathrm{~s}^{[318]}$. Alternative configurations for OF-CEAS include Brewster angle plate injection into a linear confocal cavity ${ }^{[319]}$ and a triangular ring cavity ${ }^{[320]}$. The superior robustness of OF-CEAS is demonstrated by the development of instruments for airborne measurement of atmospheric methane ${ }^{[321]}$ and in situ measurement of volcano emissions ${ }^{[322]}$.

A technique known as noise immune cavity-enhanced optical heterodyne spectroscopy (NICE-OHMS) has been developed, also known as cavity enhanced frequency modulation spectroscopy (CEFMS), reviewed by Foltynowicz et $a{ }^{[266]}$. The modulation frequency is set equal to the FSR of the cavity and the laser emission is locked to a cavity mode, such that the central carrier and both side-bands (resulting from the high frequency modulation) are transmitted through the cavity. By tuning the cavity length using a PZT actuator, the laser wavelength is also tuned as a result of the servo mechanism 
locking it to a cavity mode. The modulation must also be actively locked during the tuning process, as it has the side-effect of causing small changes to the FSR.

Why is this technique "noise immune"? For most cavity-enhanced techniques, there is a statistical uncertainty in the measured intensity in any one cavity mode, caused by laser frequency noise. However, in NICE-OHMS the fluctuations in the carrier are equally present in the side-bands as they are similarly matched to cavity modes; in this respect the measurement is self-referenced. Demodulation of the FM signal recovers the gas absorption unaffected by this form of noise. So long as other noise sources are also minimised, the technique is capable of delivering measurements close to the fundamental shot noise limit ${ }^{[266]}$.

NICE-OHMS has been used for "Doppler-free" (low pressure, ultra narrow linewidth) spectroscopy, in which counter-propagating beams are used within a linear cavity, but this can exacerbate optical interference fringes. By implementing NICE-OHMS in a ring cavity to suppress such etalons and by removing other baseline effects, Bell et al have demonstrated methane detection in a cell with $L_{\text {eff }}$ $2.3 \mathrm{~km}$ and an $\alpha_{\min }$ of $4 \times 10^{-11} \mathrm{~cm}^{-1} \mathrm{~Hz}^{-1 / 2[323]}$.

Finally, there have been various developments involving optical frequency combs. Gherman and Romanini showed that it was possible to align and mode-lock the frequency comb emission lines with the modes of a high finesse cavity, by fine scale alteration to the cavity length ${ }^{[24]}$. They used a conventional CCD spectrometer to analyse the transmitted light and demonstrated measurement of the spectrum of acetylene in the $858-862 \mathrm{~nm}$ region. By using a low noise balanced receiver, Foltynowicz et al have improved the detection sensitivity to give an $\alpha_{\min }$ of $1.7 \times 10^{-12} \mathrm{~cm}^{-1}$ per spectral element, with a 400 s acquisition time for a full spectrum ${ }^{[324]}$.

\subsection{High finesse optical fibre cavities}

There has been great interest in the use of gas cells in which the high finesse cavity is created within optical fibre, either using high reflectivity elements such as fibre Bragg gratings or by looping the fibre to form a ring resonator ${ }^{[47]}$. A schematic diagram of the latter is shown in Figure 52.

Stewart et al developed the first fibre loop cavity for gas detection coupled to a micro-optic gas cell with a pathlength of $5 \mathrm{~cm}^{[325]}$. Because of relatively high losses in the loop (compared to free space CRDS), an erbium doped fibre amplifier (EDFA) was used in combination with a variable attenuator, the latter providing fine adjustment of the loop gain so as to control the ringdown time. Ringdown times were extended to a tens of $\mu \mathrm{s}$ by the use of tens of metres of optical fibre within the loop and gain control, however it was difficult to maintain cavity stability over time ${ }^{[326]}$. In another study by von Lerber and Sigrist ${ }^{[327]}$, the high finesse fibre cavities were sensitive to bending losses in the fibre, confirming one of the practical difficulties in stabilising the system over time. Another problem is the high level of ASE noise introduced by the EDFA, causing accuracy problems similar to those seen in free space systems. $\mathrm{Ni}$ et al have used a digital adaptive filter to reduce the ASE noise, improving their system sensitivity by a factor of 8 and giving a limit of detection of $100 \mathrm{ppm}$ acetylene at $1.531 \mu \mathrm{m}$ (pathlength not stated) ${ }^{[328]}$. 


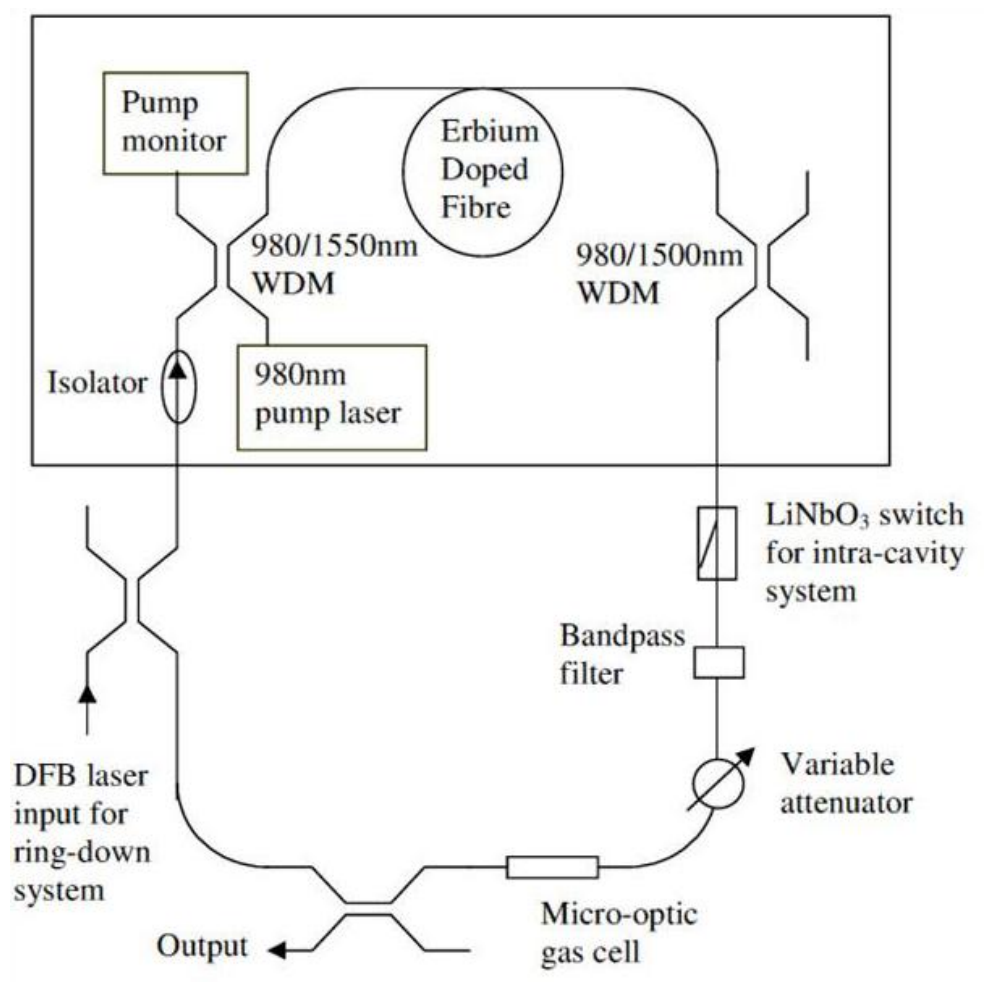

Figure 52. Schematic diagram of a fibre ring resonator implementation of CRDS. The erbium doped fibre amplifier and variable attenuator together allow the cavity finesse to be maintained despite high (and potentially variable) losses in the loop. Taken from [325].

\section{Photoacoustic detection}

Photoacoustic spectroscopy (PAS) of gases has been covered in excellent reviews by Elia et a ${ }^{[329]}$, and Zoltan et al ${ }^{[330]}$. Photoacoustic sensors differ in the way that the absorbed light is detected. In traditional transmission sensors, we monitor the level of absorbed light by comparing the light intensity in the presence and absence of gas absorption, according to equation (1). In photoacoustic sensors, the absorbed light is measured directly, as illustrated in Figure 53. Light energy absorbed by an analyte is converted to heat; for gases this generally occurs via molecular collision-induced nonradiative relaxation of excited states. The temperature increase causes the analyte and surrounding matrix (gas, liquid or solid) to expand. If the light is chopped or modulated, the expansion produces pressure waves that can be detected using a microphone ${ }^{[331]}$.

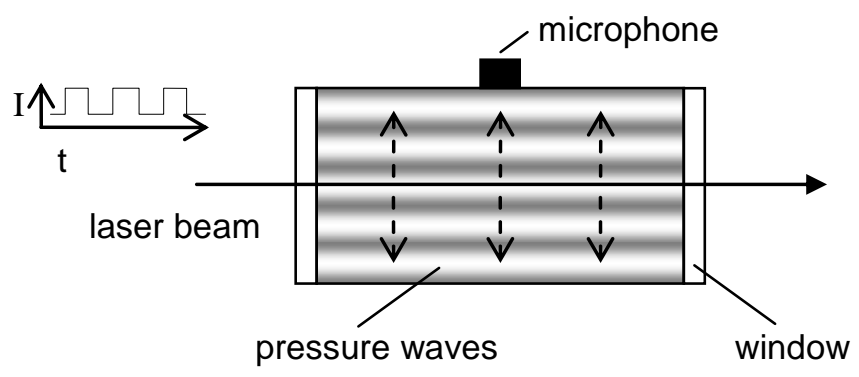

Figure 53. Detection of the photoacoustic effect in a closed cell. The pressure waves are not to scale; typically acoustic wavelength $\geq$ cell dimensions. 
The advantages are:

(i) The detected signal is directly proportional to the intensity of light absorbed, $\Delta I$, rather than to that transmitted, $I$. The effects of shot noise, flicker noise and relative intensity noise (associated with the light source) are greatly reduced. For the same reason, in TDLS the relative magnitude of interference fringes can also be reduced.

(ii) Scattered light, which in conventional spectrophotometry would reduce the effective value of $I_{0}$ thus giving a spurious absorption measurement, has less effect on the photoacoustic signal ${ }^{[332]}$.

(iii) The dynamic range of the technique can be large.

(iv) No photodetector is required for the main signal, which can improve performance in the longer wave mid IR where standard uncooled detectors have poor detectivity. Although a photodetector may still be required to normalise the signals against incident power, the effects of detector noise are reduced when we combine this with point (i).

A disadvantage is that if the background matrix changes, relaxation processes and pathways can change, with a consequent alteration in the signal level. For example, for detection of methane in air there is interaction between methane and oxygen with a fast transfer of energy from excited methane to oxygen (faster than the modulation frequency), and a slow relaxation of oxygen molecules (not detectable at typical modulation frequencies). This process significantly reduces photoacoustic signal strengths ${ }^{[333]}$. The key point is the time constant of the energy relaxation pathway in relation to the time of one modulation cycle. Hence at increased sample pressures, PA signal generation can be more efficient because more frequent collisions increase the likelihood of energy transfer from molecular vibration to translation (V-T relaxation) within any modulation cycle ${ }^{[334]}$.

Photoacoustic detection can be applied in combination with any of the spectroscopies mentioned in this article, and in the right circumstances can deliver an order of magnitude or more improvement in the limit of detection. Because the received signal is usually proportional to the excitation power $I_{o}$, and the limiting noise is independent of $I_{0}$, system performance is often compared using a normalised figure of merit based on the minimum detectable absorbance $\alpha_{\min }$, namely $\alpha_{\min } \cdot I_{o}$ in units of $\mathrm{cm}^{-1} \mathrm{~W}$.

A disadvantage of photoacoustic systems is that they are highly sensitive to background acoustic noise and vibrations. In field use, this can be the performance-limiting feature, yet can vary from site to site and is hard to predict. For other measurements, this noise would be reduced by increasing the modulation frequency $f$, as the magnitude of background vibrations is generally inversely proportional to frequency ${ }^{[330]}$. However, the photoacoustic signal, resulting from integration of the energy absorbed during each modulation cycle, is also proportional to $1 / f$, so the general result is no net improvement in signal to noise ratio.

To avoid background acoustic noise, a number of designs use a non-absorbing reference channel which can be positioned in a differential geometry. Provided that the reference channel is located close to the sample channel (displaced by less than the acoustic wavelength for the modulation frequency used), this can experience background vibrations to the same extent as the sample channel and their effect can be minimised.

Acoustically resonant systems have been used extensively to amplify the acoustic signal; Figure 54 shows a number of different geometries in use. This can provide a large signal enhancement; one example based on a quartz resonator has a $Q$ factor of over $10,000^{[335]}$. Care must be taken if the limiting noise is background acoustic noise, in which case the noise might be amplified by an equally 
large amount. However, it is possible to place potential sources of such noise at pressure nodes, rather than antinodes, to suppress excitation of the background at the resonant frequency. Such sources can include gas inlets and windows, which can generate pressure signals at the modulation frequency as a result of fractional absorption within the window material.

a)

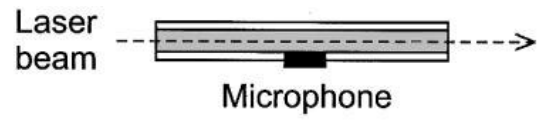

b)

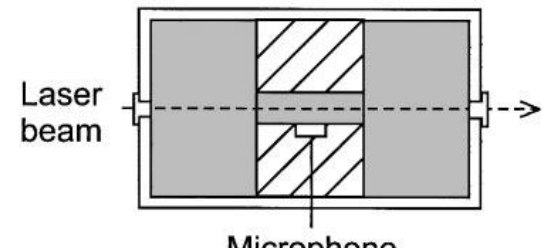

c)

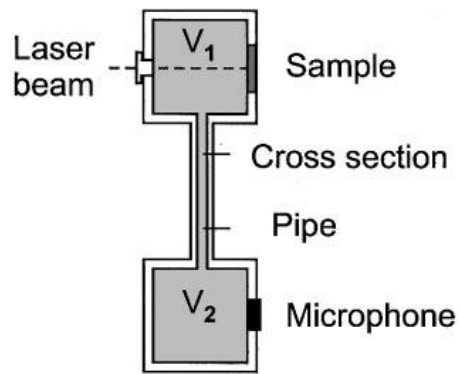

d)

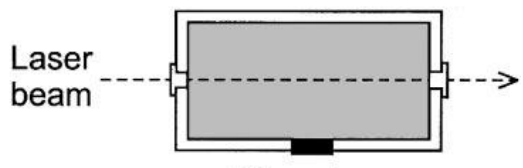

Microphone

Microphone

e)
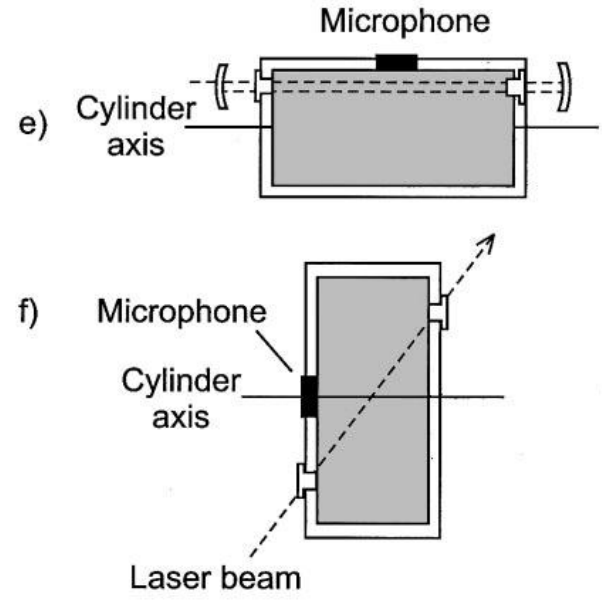

Figure 54. Schematic designs for different types of resonant photoacoustic cell. (a) Simple organ pipe resonator, (b) organ pipe with buffer volumes at each end, (c) Helmholtz resonator with sample and reference chambers (configured in this case for solid samples), (d) closed cylinder with excitation of coaxial resonant modes, (e) closed cylinder with excitation of azimuthal resonant modes, (f) cylindrical cell with suppression of window noise. Taken from [336].

Kosterev et al have pointed out that, in the linear region of the Beer Lambert law (equation (1)), the signal $S$ from a resonant photoacoustic cell is equal to ${ }^{[334] \text { : }}$

$$
S=\eta \frac{\alpha \ell C I Q}{f V}=\eta \frac{\alpha C I Q}{f A}
$$

Where $I$ is the optical power in W, Q is the quality factor of the resonant cell, $\mathrm{V}$ is the cell volume, $\mathrm{A}$ is the cross-sectional area, $f$ is the modulation frequency and $\eta$ is a system efficiency factor that takes into account the microphone transfer function and other potential loss factors. It can be seen from equation (12) that the signal is no longer a function of optical pathlength, therefore the latter can be reduced without affecting the signal to noise ratio. For a resonant system, smaller dimensions would require a higher modulation frequency, but we have already seen that if the limiting noise is due to background acoustic vibration, the higher frequency might not result in a deterioration in signal to noise ratio.

Resonant cells with a high $Q(>500)$ require active temperature stabilisation in order to maintain the resonance, otherwise temperature - induced variation of the velocity of sound in air will allow the system to drift away from the resonant peak. Miklós et al calculated that, for responsivity to drift by 
less than $1 \%$, the temperature change $\Delta T$ must be kept within $Q \cdot \Delta T \leq 56$, and $Q \cdot \Delta T \leq 138$ for a $10 \%$ drift $^{[336]}$.

Background acoustic noise can be reduced by making use of the phase difference between the background noise and signal in resonant systems, ensuring pressure nodes correspond to inlets for gases $^{[337]}$, supports and the location of windows ${ }^{[330]}$. Saarela et al further used a differential microphone arrangement, giving signals $180^{\circ}$ out of phase from opposite ends of the cell, as shown in Figure 55, and measuring the difference between the two. In this way, acoustic noise from other sources affecting the microphones equally was suppressed. Window noise can be reduced by use of wavelength modulation, since absorption by windows is normally a broadband effect that affects the entire baseline ${ }^{[329]}$.

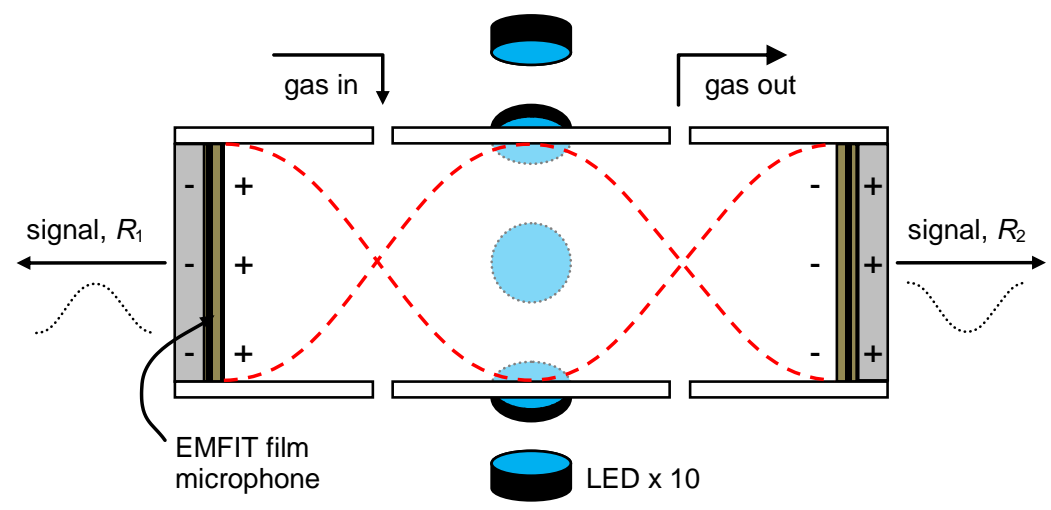

Figure 55. Differential microphone arrangement of Saarela et al, after [337]. The dashed line corresponds to the amplitude of standing pressure waves in the cell.

A conventional resonant cell has been miniaturised by Gorelik and Starovoitov ${ }^{[338]}$. Although apparently simple (see Figure 56), its design is based on a detailed consideration of resonant acoustic modes. The position of the microphone and windows has been optimised so as to maximise the PA signal magnitude at the microphone and minimise spurious background effects. With an incident power of $66 \mathrm{~mW}$, a normalised $\alpha_{\min }$ of $3.2 \times 10^{-8} \mathrm{~cm}^{-1} \mathrm{~W} \mathrm{~Hz}^{-1 / 2}$ was demonstrated with a signal averaging time of $0.13 \mathrm{~s}$.

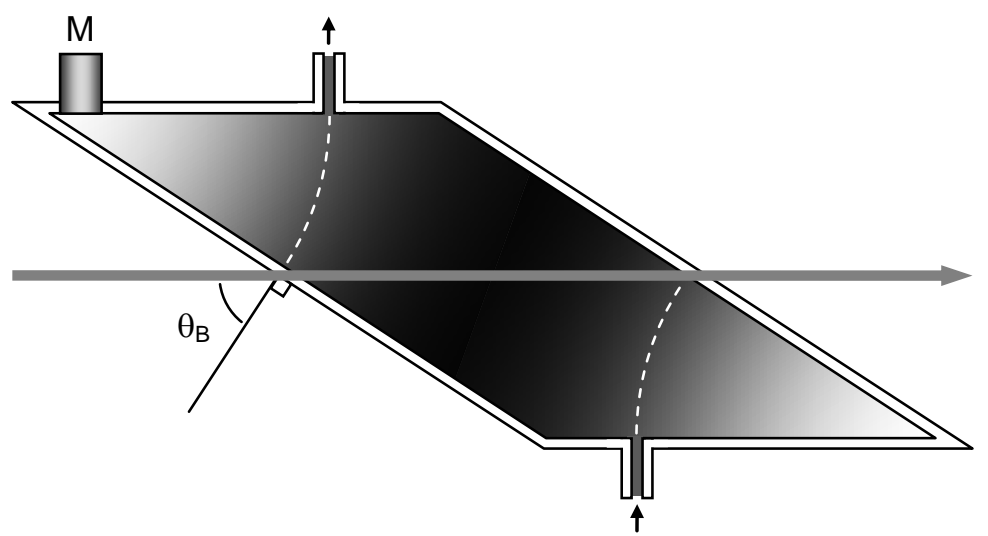

Figure 56. Miniature resonant photoacoustic cell designed by Gorelik and Starovoitov, with an optical pathlength of $1 \mathrm{~cm}$. Shading indicates the amplitude of pressure waves in the resonant acoustic mode. Windows were placed at the Brewster angle $\theta_{B}$ to the optical path and the microphone was placed at position M. After [338]. 
The quartz-enhanced PAS (QEPAS) system of Rice University was also designed with acoustic noise suppression in mind. The sensor including optical pathlength is very small (a few $\mathrm{mm}$ in length) and can be located within a larger sample volume. The quartz tuning fork (QTF) at the heart of the sensor is a quadrupole, conferring excellent immunity to externally generated noise, which tends to move the prongs of the tuning fork in a similar direction and therefore is less likely to induce quadrupole vibrations. An example of such a QTF is shown in Figure 57. The piezoelectric current generated by the QTF is detected using a transimpedance amplifier, giving a normalised $\alpha_{\min }$ in the range $10^{-8}-10^{-9} \mathrm{~cm}^{-1} \mathrm{~W} \mathrm{~Hz}^{-1 / 2}[334,335]$. The QEPAS sensor has been demonstrated in detection of many different gases (see multiple references in ) using tunable lasers including near IR DFB and VCSELS, QCLS and DFG sources.

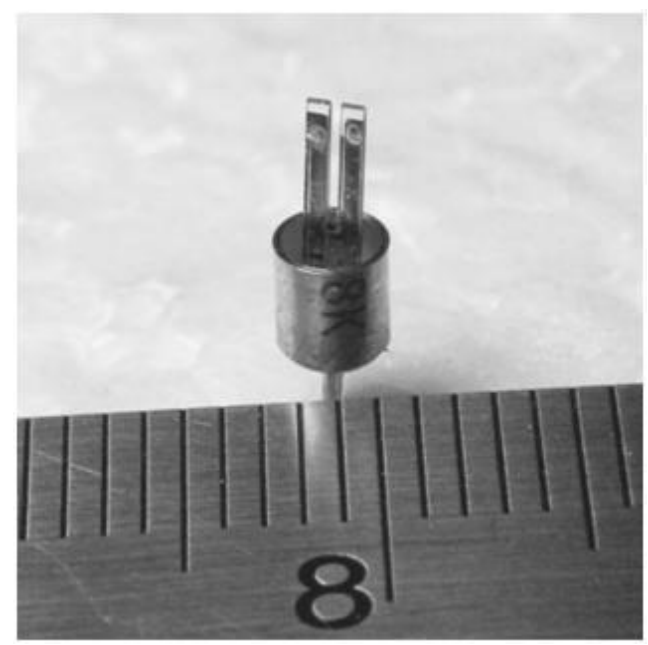

Figure 57. Typical quartz tuning fork as adapted by Rice University for PAS, shown next to a scale marker ruled in $\mathrm{mm}$. Taken from [334].

To improve sensitivity, a number of groups have developed microcantilevers as an alternative to microphones. The microcantilevers have very low mechanical compliance and show large deflections in response to pressure waves, their displacement detected optically via deflection of a reflected laser beam ${ }^{[339]}$ or interferometrically ${ }^{[340]}$. The latter system used a differential geometry that conferred some immunity to background noise, giving a normalised $\alpha_{\min }$ of $1.7 \times 10^{-10} \mathrm{~cm}^{-1} \mathrm{~W} \mathrm{~Hz}^{-1 / 2}$ when used for detection of $\mathrm{CO}_{2}$ at $1.572 \mu \mathrm{m}$. Recent miniaturisation of the latter system has led to the device pictured in Figure 58.

Lindley et al have completed an intercomparison of three different PA cell designs, ensuring that the three cells were used under similar laboratory conditions and with similar excitation sources ${ }^{[341]}$. The three designs were a simple resonant tube similar to that in Figure 54 (a), a differential cell based on resonant flow tubes and the microcantilever system of reference [340]. Their work confirmed the superior sensitivity of the microcantilever design, with normalised $\alpha_{\min }(1 \sigma)$ values of $1.6 \times 10^{-7}$, $8.5 \times 10^{-8}$ and $1.1 \times 10^{-9} \mathrm{~cm}^{-1} \mathrm{WHz}^{-1 / 2}$ obtained respectively.

Lindley et al also investigated improvements to PA sensitivity by using an erbium doped fibre amplifier (EDFA) to increase the incident power from a few $\mathrm{mW}$ to over $1 \mathrm{~W}$. Limits of detection for acetylene were improved for all PA cell types, for example by a factor of 152 with the microcantilever cell for a power increase from $1.5 \mathrm{~mW}$ to $1.2 \mathrm{~W}$ (a factor of $10^{3}$ ). However, in all cases the improvement in performance was lower than the proportional increase in power, and normalised values of $\alpha_{\min } I_{0}$ 
deteriorated. The authors attributed this to several factors including increased ASE noise from the EDFA, possible saturation effects, and a possible deterioration in beam quality. Saarela et al simply increased the incident light power from their LED sources by directing as many of them as possible (10 in total) to the centre of their resonant cell. Further techniques to improve sensitivity include the use of a resonant photoacoustic cell placed within a multipass Herriott cell, such that the laser beam was required to pass through the cell windows with every pass ${ }^{[342]}$, and use of an open-ended resonant tube placed within a confocal cavity ${ }^{[343]}$.

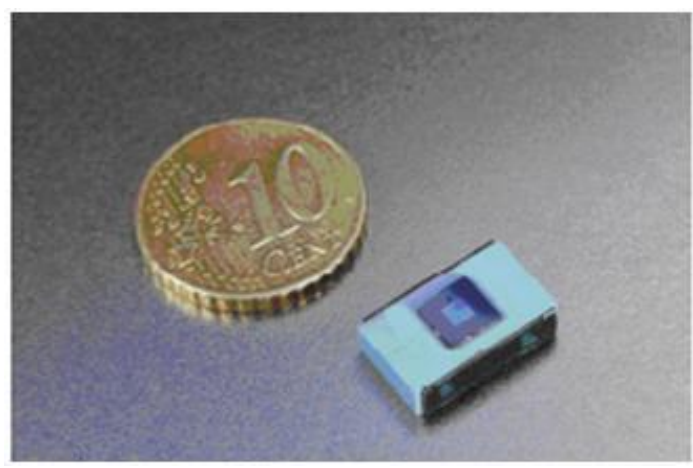

Figure 58. Differential photoacoustic cell incorporating a microcantilever sensor, designed to be addressed interferometrically via optical fibre (not shown). The scale marker is a Euro ten cent coin

(20 mm diameter). Taken from [344].

Photoacoustic spectroscopy has been used by Varga et al in combination with TDLS at $1.57 \mu \mathrm{m}$ to measure $\mathrm{H}_{2} \mathrm{~S}$ in natural gas process streams ${ }^{[345]}$, based on a differential resonant PA cell designed by Miklos et $a^{[336]}$ (see Figure 54 (c) for a single, non-differential example). The results of laboratory tests have been reported, with a limit of detection of $0.16 \mathrm{ppm}$. This good performance was established by comparing measurements from gas pipelines containing sour gas (with $\mathrm{H}_{2} \mathrm{~S}$ ) and sweetened gas $\left(\mathrm{H}_{2} \mathrm{~S}\right.$ removed), and by use of piped zero reference gases to prevent short-term drift effects. This provided an almost perfect reference, to correct for matrix interfering chemical species as well as environmental changes and acoustic noise that could also affect the measurement. Successful operation on a natural gas processing plant was also reported for an 18 month period, during which time the system was claimed to run "totally unattended". Although performance data for this period were not explicitly stated, agreement with alternative methods of $\mathrm{H}_{2} \mathrm{~S}$ measurement was within $0.5 \mathrm{ppm}$; to maintain this level of performance in a noisy environment is an achievement.

\section{Comparison of different gas detection techniques}

The difficulties in comparing different techniques have been nicely discussed by Moyer et al ${ }^{[300]}$. Almost inevitably, a figure of merit that makes sense in one application is of limited value in another, or difficult to calculate. Within this article, we have attempted to compare performance using the common metrics of NEA and $\alpha_{\text {min. }}$. However, a brief summary prevents us from factoring in other needs such as response time requirements, the need to avoid cross-interference to a particular species in certain applications, the ability to reduce sample pressure to use narrower linewidths, limits on cost, complexity or space. Furthermore, normalising these metrics to the measurement bandwidth in $\mathrm{Hz}^{1 / 2}$ makes an assumption that limits of detection will be improved by increasing the averaging time; this is 
true up to a point determined by drift effects. For detailed discussions on these issues for particular systems, the reader is directed to the original papers.

Practitioners usually find that performance achieved in the laboratory is rarely replicated in the field. What is the scientific basis for this? In optical systems there are a number of possibilities. It can be difficult to maintain the precise alignment needed, especially in systems with long equivalent pathlengths. Uncontrolled or unknown variations in temperature and pressure within the sample can affect accuracy by altering the linewidth $y$ and absorption strength $S$ in equation (3), and matrix effects can also alter $\mathrm{y}$. Finally, optical interference fringes can affect any of the spectroscopic techniques described in this article, but particularly TDLS. Fringes on the zero baseline will drift as a result of thermal expansion or contraction of the responsible cavity. As optical gas detection often involves measuring changes from the baseline rather than absolute measurements, fringe drift is unlikely to affect laboratory measurements made within a few minutes, but can cause a marked deterioration in performance over longer time periods, especially for large or rapid temperature excursions. Methods to reduce such deterioration include active temperature stabilisation of the instrument ${ }^{[346]}$ and frequent re-zeroing the instrument with clean air (sometimes known as "zero air") ${ }^{[228]}$, all of which comes at a cost to instrument complexity.

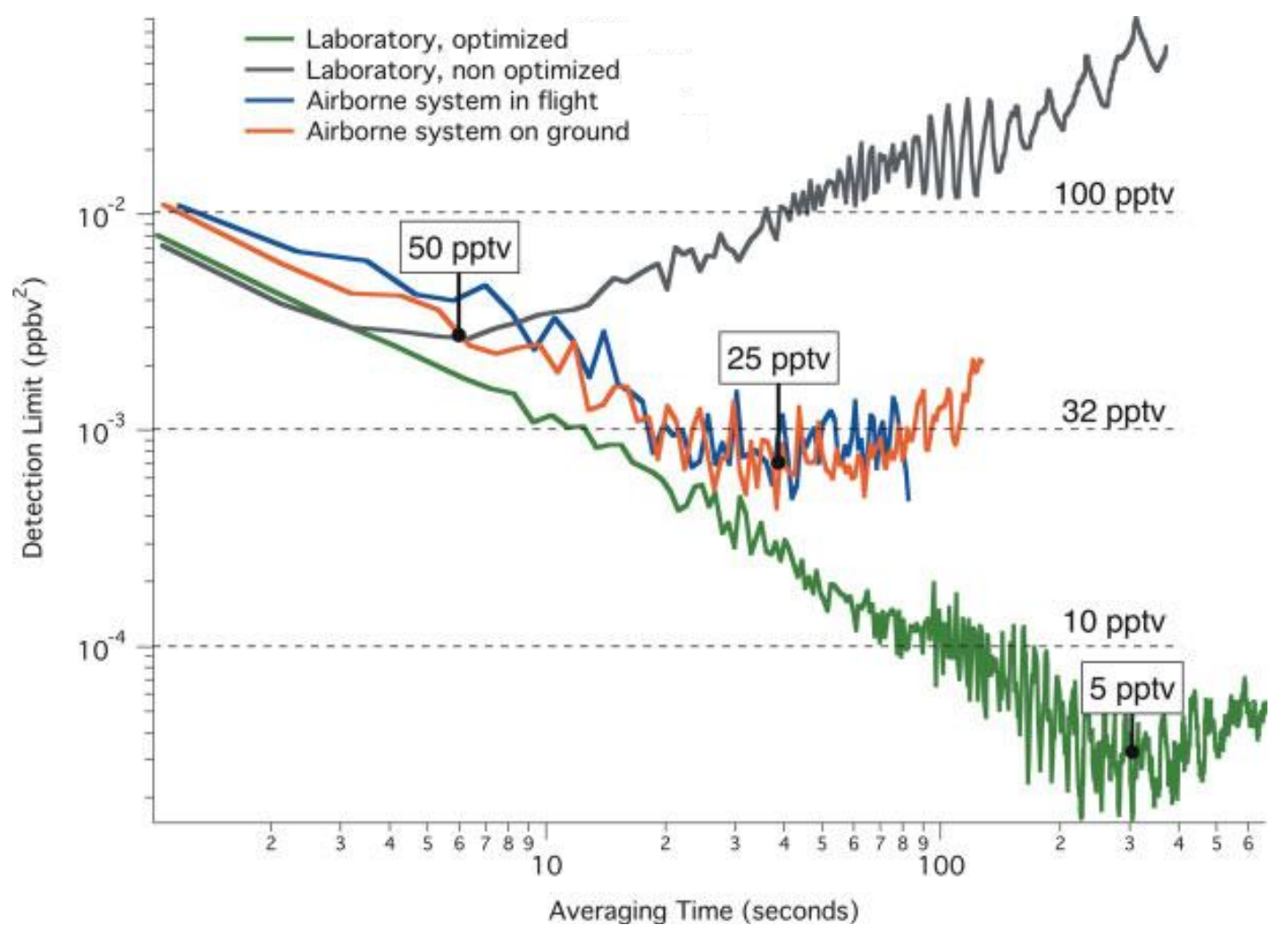

Figure 59. Allan variance for different configurations of a TDLS based instrument designed for airborne measurement of atmospheric formaldehyde. Taken from [228].

An Allan variance plot is often used to visualise the effect of signal averaging and drift ${ }^{[347]}$. This plot shows the variance $\left(\sigma^{2}\right)$ or standard deviation $(\sigma)$ in optical signals as a function of averaging time for the instrument. An example is shown in Figure 59 for a TDLS based system designed to measure formaldehyde in the atmosphere from onboard aircraft - a challenging environment, owing to wide 
excursions in both temperature and pressure of the external environment, vibration of the aircraft itself, and constraints on the size, weight and power of the system. As expected, at shorter averaging times the measurement is limited by white noise and therefore the variance is inversely proportional to averaging time. However, after some optimum averaging time, drift effects can be seen as a rise in variance as averaging time is increased. The point at which this happens, and the extent to which performance deteriorates thereafter, are both application- and installation- specific for a given instrument. As Figure 59 shows, the difference in limit of detection between an optimised laboratory instrument and an airborne instrument in flight is a factor of 5 . The authors noted that re-zeroing with zero air once a minute often improved the performance ${ }^{[228]}$.

Notwithstanding our disclaimer above, we have compared the performance of broad categories of optical gas detection techniques in terms of both NEA and $\alpha_{\text {min. }}$. Plots of these figures of merit versus optical pathlength for different systems are shown in Figure 60; the plots are based on data from the references in this article, including in the appendix, and therefore represent real, demonstrated experimental performance.

What can we learn from this analysis? The following observations can be made.

- Broadband measurements (NDIR, BB-CEAS) are confirmed to suffer worse signal to noise ratios than equivalent narrowband techniques, and the long pathlength spectroscopy of DOAS has performance that lies between broadband measurements and very high resolution, tunable laser measurements, as we might expect.

- The techniques with the lowest $\alpha_{\min }$, ie those best able to detect low gas concentrations, are those for which very long pathlengths (up to tens of $\mathrm{km}$ ) can be established, namely tunable laser based, cavity-enhanced techniques.

- One might have expected that techniques with longer pathlengths would show a deterioration in NEA owing to the increased optical complexity of the system (ie they might be more likely to exhibit troublesome interference fringes). The evidence here does not support this in general, but the trend appears to hold within a number of individual categories. It may be that the best instruments have been designed to avoid interference fringes through a combination of balanced detection and thermal management. It is also true that performance limits are often reported for short-term measurements, whereas the effect of fringes will cause drift over the longer term.

- For the short optical pathlengths used, PAS shows very good performance compared with other techniques; this may well be due to the pathlength invariance of equation (12) already discussed.

- When comparing $2 f$ WMS and TTFMS, no overall difference in performance was observed, therefore these categories were combined into one. This may be, as discussed in section 6.2, because of improvements in the relative intensity noise of laser diodes since TTFMS was first devised.

- One might have expected a difference in performance between directly scanned TDLS and $2 f$ WMS, but at the lower end of the NEA and $\alpha_{\min }$ scales, the evidence shows that these techniques perform similarly. As previously discussed, the best performance for each technique is due to the use of balanced detection systems that compensate for laser intensity fluctuation and certain types of interference. If both techniques are limited in practice by optical interference fringes, their limits should be the same. It is possible that direct TDLS would also be limited by the bit resolution of 
data acquisition systems, however the availability in the last few years of digital / analogue converters with 18 bit or greater resolution may have removed this obstacle.

- There is a plethora of recently emerged cavity-enhanced techniques, which have been categorised separately because of the field's relative immaturity compared to the others here. Of the cavityenhanced techniques, OA-ICOS appears to be demonstrating excellent performance and has the advantage of being one of the simpler cavity-enhanced techniques to implement.

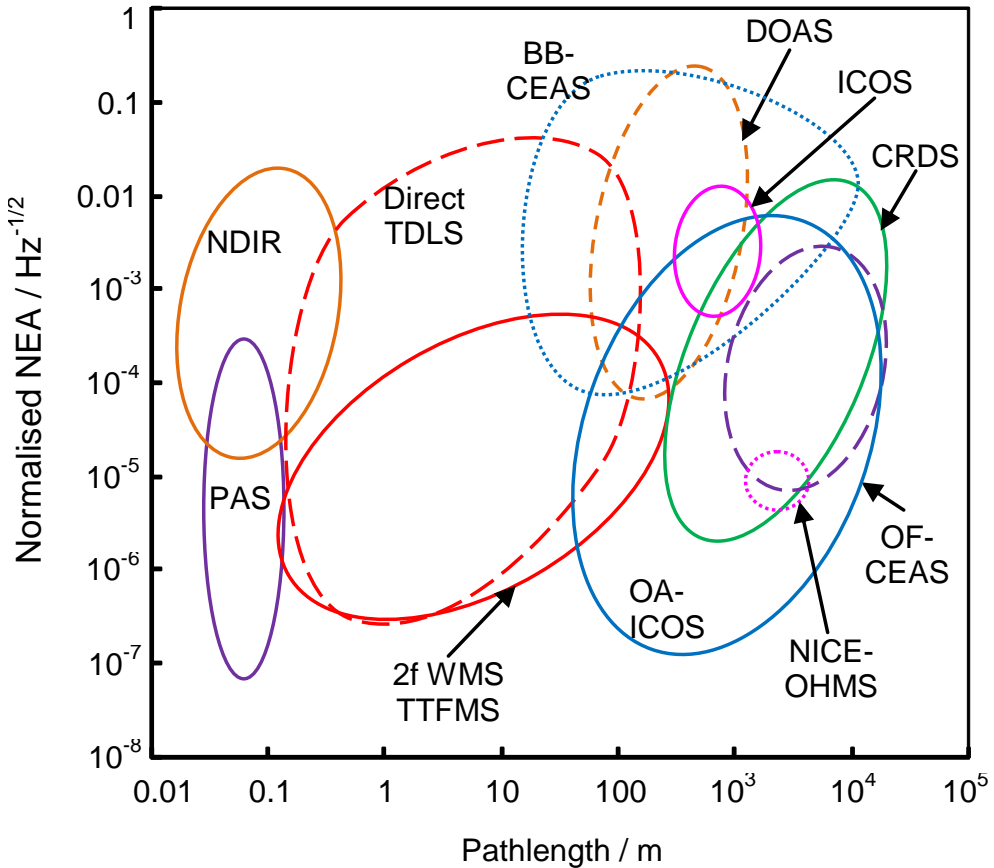

(a)

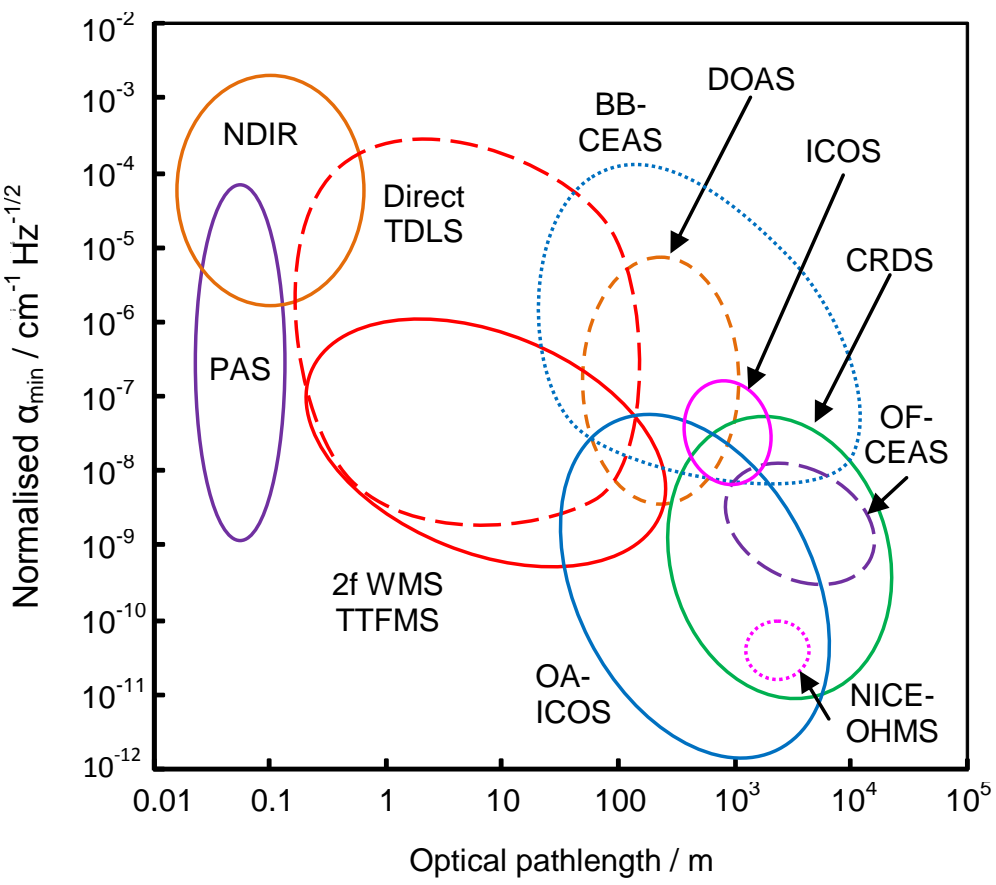

(b)
Key: BB-CEAS - broadband cavityenhanced spectroscopy, CRDS - cavity ringdown spectroscopy, direct TDLS directly scanned tunable diode laser spectroscopy, DOAS - differential optical absorption spectroscopy, $2 f$ WMS $-2^{\text {nd }}$ harmonic wavelength modulation spectroscopy, ICOS - integrated cavity output spectroscopy, OA-ICOS - off-axis ICOS, OF-CEAS - optical feedback CEAS, NDIR - non-dispersive infrared, NICEOHMS - noise-immune cavity-enhanced optical heterodyne spectroscopy, PAS photoacoustic spectroscopy, TTFMS two-tone frequency modulation spectroscopy.

Figure 60. Summarised performance of different categories of gas detection techniques as a function of optical pathlength: (a) NEA, (b) $\alpha_{\min }$. 


\section{Discussion and conclusions}

One of the technical compromises in optical gas sensing is to work at very high resolution with limited wavelength coverage, or to make measurement over a wide range of wavelengths but with a worse signal to noise ratio. The same is true of review articles; in an article of this breadth we cannot cover any one area with sufficiently high resolution to do it justice, and neither can we report on every clever advance that has been reported in the field. We can only hope not to have missed anything of great significance, and apologise where we have done so. Instead, we hope to gain by drawing out common themes and comparisons across the field. And fortunately there exists a number of excellent review articles able to probe more deeply into the individual techniques described here. A second problem with measurements is the response time; as soon as a measurement has been reported, it is out of date, and in general the larger the sample size, the longer the response time. The response time of this article has been well over a year owing to its large sample size, plus a sampling delay time of a few months for the review and publication process.

From section 9, it is obvious that the dynamic range of "optical gas sensing" is large. We haven't considered upper limits of detection of any of these techniques, yet the lower limit of detection, defined by the $\alpha_{\min }$ parameter, covers nine orders of magnitude, with instruments ranging from a simple source, pathlength and detector (NDIR) to complex laser systems under active control. The range of applications is consequently very large for a common signal transduction mechanism, and a number of those applications have been mentioned throughout this article.

A number of general trends can be identified going forward. Firstly, the mid IR region has opened up to TDLS with the availability of turnkey, room temperature laser diodes now covering almost the entire range from 1.3 to 11 or $16 \mu \mathrm{m}$, depending on whether we consider pulsed or CW lasers. This is having a knock-on effect on the availability of supporting mid IR technology, from optical fibre to diagnostic instruments for visualising beams or resolving emission spectra at high resolution; previously, such technology was largely developed for IR imaging applications. At the same time, technology developed originally for the telecommunications industry at around $1.55 \mu \mathrm{m}$ is extending its reach into the 2-3 $\mu \mathrm{m}$ region and even up to $3.5 \mu \mathrm{m}$. For certain components, such as multi-channel detectors for NDIR, device integration and improved packaging have facilitated more widespread uptake of the gas detection technique. Integration and packaging of lasers, photodetectors and associated optics might therefore be an area ripe for further development, taking know-how from the telecommunications industry.

Several developments are helping to close the gap between spectrometers with broad wavelength coverage but limited resolution / signal to noise ratios, and narrow band, ultra high resolution measurements made using tunable lasers. With external cavity systems, the wavelength coverage of the latter is increasing, and recent developments in dual frequency comb based Fourier transform spectroscopy may widen coverage still further. At the same time, many novel optical techniques have been applied to the problem of improving spectrometer performance, as previously described in section 5 . It is possible that some consolidation may result, with one or two key technologies gaining commercial significance, perhaps even one day replacing the FTIR in both the lab and the field.

Cavity-enhanced techniques have developed rapidly over the last decade and there is a wide variety of different techniques to choose from. The precise choice of technique may currently be dictated by 
the characteristics of whatever laser is available to cover the wavelength region of choice. However, as mid IR lasers develop further, we may also see consolidation in this field.

Finally, several small photoacoustic devices have recently been developed. Their small size has advantages for system response time and is potentially an advantage in itself for example in portable equipment. However, at present the overall size of any instrument would be dominated by the physical size of the laser focusing optics and other associated optics. It might be that improvements in packaging of the latter is needed to realise the full benefits of using such small optical cells.

Using optical absorption offers a number of advantages that alternative technologies cannot provide. Because measurement are based on a fundamental physical property of the target molecule, some mechanisms of failure associated with materials based technologies are avoided, including poisoninginduced drift, lack of selectivity and batch-to-batch variation in responsivity. Optical gas measurements are usually self-referenced, which means that it is possible to design auditable instruments that "know" whether they have failed (eg by a failure of a light source or a failure to direct sufficient light in and out of the sample). Finally, the rapidity of the signal transduction, and the fact that the measurement can be made in situ, means that optical instruments can have a significantly faster speed of response than alternative technologies (the highest reported detection bandwidth in $\quad$ is $1.2 \mathrm{MHz}$, which is unusual, but response times of 1 s are common).

Arguably the biggest disadvantage of optical gas detection is its cost; compared to materials based or electrochemical sensors, they are significantly more expensive, even for the simplest NDIR sensor. For many one-off applications, where there is no alternative method, this does not matter. Use in industrial environments and field based measurement remains a challenge, especially where there are also cost constraints. Fortunately there exists a large number of niche applications for which optical systems make both scientific and commercial sense. A second disadvantage is also a strength; a high degree of selectivity also means that multiple gas species are hard to detect simultaneously without a proportional increase in system complexity. Finally, by using optical absorption, we have to be led by the information content available in an absorption spectrum. There are some gases that are more challenging to measure: those without a permanent dipole (eg $\mathrm{H}_{2}, \mathrm{~N}_{2}, \mathrm{O}_{2}$ ) with insignificant infrared absorption, and those that happen to have small absorption coefficients $\left(e \mathrm{H}_{2} \mathrm{~S}\right)$. A second challenge is to be able to measure a wider range of gas compounds such as larger molecules including volatile organic compounds (VOCs), which have broader spectra with lower information content, requiring wider wavelength coverage and greater reliance on spectral fitting algorithms. The problem of access to interesting parts of the spectrum is now easing greatly and opening up many new applications.

To conclude, optical gas sensing remains an important field that complements other gas detection technologies. The opportunities afforded by new technology, together with the challenges that remain, will make this an exciting and rapidly developing field for many years to come. 


\section{Appendix Performance comparison of optical gas detection techniques}

We have completed a survey of recent published results for the detection of a number of gas species and summarized these in Table 8. We note the following facts concerning the survey.

1. In this table, we report on a survey of recently reported developments in optical absorption of a range of gases, namely ammonia $(\mathrm{NH} 3)$, benzene $(\mathrm{C} 6 \mathrm{H} 6)$, carbon dioxide $(\mathrm{CO} 2)$, carbon monoxide (CO), ethane $(\mathrm{C} 2 \mathrm{H} 6)$, formaldehyde $(\mathrm{H} 2 \mathrm{CO})$, hydrogen sulfide $(\mathrm{H} 2 \mathrm{~S})$, methane $(\mathrm{CH} 4)$, nitric oxide (NO), nitrous oxide (N2O), nitrogen dioxide (NO2), sulphur dioxide (SO2) and water vapour (H2O). We chose this list somewhat arbitrarily, however aiming for a balance between gases of concern in environmental, safety and industrial applications, and choosing those with sufficient reported activity to make a comparison table worth producing.

2. Techniques were surveyed over the preceding 5 - 10 years using the Thomson Reuters and Scopus databases. Where few papers were found for a given gas, the timescale was extended. Examples are presented showing detection capability for different techniques; where more than one paper was found that refers to the same technique, to avoid repetition the best reported performance was included (in many cases, repeated papers have reported improvements to a technique by the same team).

3. Where possible, different studies have been compared using the minimum detectable absorbance (in absorbance units, $\mathrm{AU}$ ) and the minimum detectable absorption coefficient (in $\mathrm{cm}-1$ ), in all cases stating the $1 \sigma$ values. Where reported by authors themselves, these have been quoted normalised by the measurement bandwidth in $\mathrm{Hz} 1 / 2$. Where we have calculated figures of merit (for example calculating the minimum detectable absorbance using the authors' stated minimum detectable absorption coefficient and stated pathlength), the result is given in italics.

4. In the case of photoacoustic techniques, the incident power in $\mathrm{W}$ has been stated and detection limits normalised to an incident power of $1 \mathrm{~W}$, in the expectation that signal to noise ratios are proportional to that incident power. This is standard practice in the field and most authors report in this manner.

5. For open path measurements, detection limits are reported in the pathlength-integrated form, for example as ppm.m rather than ppm. In some applications such as urban air quality, it is rare for the gas "sample" to occupy the measured pathlength homogeneously, rather it is more typical for gas clouds to be present over shorter distances.

6. Different studies are listed first in order of the gas detected, then in order of wavelength (records at similar wavelengths are grouped together), and finally in decreasing order of minimum detection limit by concentration. Concentrations are expressed as parts per million $10^{6}(\mathrm{ppm})$, billion $10^{9}(\mathrm{ppb})$ or trillion $10^{12}(\mathrm{ppt})$, in all cases by volume.

\section{Key}

BB-CEAS - broadband cavity-enhanced spectroscopy, CRDS - cavity ringdown spectroscopy, direct TDLS directly scanned tunable diode laser spectroscopy, DOAS - differential optical absorption spectroscopy, $2 f$ WMS $-2^{\text {nd }}$ harmonic wavelength modulation spectroscopy, ICOS - integrated cavity output spectroscopy, OA-ICOS off-axis ICOS, OF-CEAS - optical feedback CEAS, NDIR - non-dispersive infrared, NICE-OHMS - noise-immune cavity-enhanced optical heterodyne spectroscopy, PAS - photoacoustic spectroscopy, QEPAS - quartzenhanced PAS, TTFMS - two-tone frequency modulation spectroscopy. 
Table 8. Comparison of performance indicators for optical detection of different gases

\begin{tabular}{l|l|l|l|l|l|l|l} 
Gas & Wave-length & Path- & Response / Min detectable & Min detectable & Min detect- & Technique
\end{tabular}

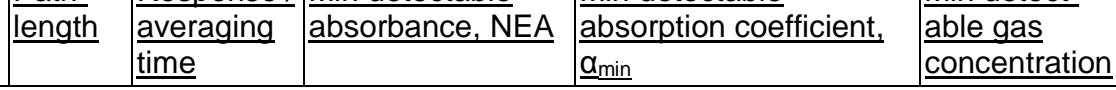

\begin{tabular}{|c|c|c|c|c|c|c|c|c|}
\hline \multirow{14}{*}{$\underset{\mathrm{NH}_{3}}{\operatorname{ammonia}}$} & $190-230 \mathrm{~nm}$ & $150 \mathrm{~m}$ & 1s & $5 \times 10^{-5}$ & $3 \times 10^{-9} \mathrm{~cm}^{-1}$ & $0.15 \mathrm{ppm} . \mathrm{m}$ & DOAS & {$[348]$} \\
\hline & $200-230 \mathrm{~nm}$ & $60.5 \mathrm{~cm}$ & 1s & Not stated & Not stated & $1 \mathrm{ppm}$ & $\begin{array}{l}\text { CCD } \\
\text { spectrometer }\end{array}$ & [349] \\
\hline & $1.532 \mu \mathrm{m}$ & $3 m$ & $6 s$ & $3.5 \times 10^{-5}$ & $1.2 \times 10^{-1} \mathrm{~cm}^{-1}$ & 7-14 ppm & \begin{tabular}{|l|}
$2 f \mathrm{WMS}$, \\
waveguides
\end{tabular} & [67] \\
\hline & $1.532 \mu \mathrm{m}$ & $36 \mathrm{~m}$ & $30 \mathrm{~s}$ & $1.4 \times 10^{-4}$ & $3.9 \times 10^{-8} \mathrm{~cm}^{-1}$ & $0.2 \mathrm{ppm}$ & Direct TDLS & [350] \\
\hline & $1.532 \mu \mathrm{m}$ & $4 \mathrm{~cm}$ & $10 \mathrm{~s}$ & $4 \times 10^{-8}$ & $\begin{array}{l}1.3 \times 10^{-10} \mathrm{~cm}^{-1} \mathrm{~W} \mathrm{~Hz}^{-1 / 2} \\
1 \times 10^{-8} \mathrm{~cm}^{-1}\end{array}$ & $50 \mathrm{ppb}$ & $\begin{array}{l}\text { Direct TDLS } \\
\text { PAS, } 40 \mathrm{~mW}\end{array}$ & [351] \\
\hline & $1.512 \mu \mathrm{m}$ & $\begin{array}{l}\text { Not } \\
\text { stated }\end{array}$ & $30 \mathrm{~s}$ & Not stated & $8 \times 10^{-10} \mathrm{~cm}^{-1}$ & $18 p p b$ & ICOS & [352] \\
\hline & $1.532 \mu \mathrm{m}$ & $5.4 \mathrm{~mm}$ & $10 \mathrm{~s}$ & Not stated & $\begin{array}{l}4.7 \times 10^{-10} \mathrm{~cm}^{-1} \\
4.7 \times 10^{-10} \mathrm{~cm}^{-1} \mathrm{~W}\end{array}$ & $2.4 \mathrm{ppb}$ & $\begin{array}{l}\text { WMS, PAS } \\
1 \mathrm{~W}\end{array}$ & [353] \\
\hline & $1.532 \mu \mathrm{m}$ & $5.0 \mathrm{~km}$ & $1 \mathrm{~s}$ & $1.4 \times 10^{-5}$ & $2.8 \times 10^{-11} \mathrm{~cm}^{-1} \mathrm{~Hz}^{-1 / 2}$ & $0.7 \mathrm{ppb}$ & OA-ICOS & [296] \\
\hline & $1.527 \mu \mathrm{m}$ & $\begin{array}{l}\text { Not } \\
\text { stated }\end{array}$ & $<60 s$ & Not stated & $8 \times 10^{-11} \mathrm{~cm}^{-1} \mathrm{~Hz}^{-1 / 2}$ & $0.4 \mathrm{ppb}$ & CRDS & [354] \\
\hline & $2.00 \mu \mathrm{m}$ & $5.3 \mathrm{~mm}$ & $1 \mathrm{~s}$ & $\begin{array}{l}4.7 \times 10^{-y} \mathrm{~W} \mathrm{~Hz}^{-1 / 2} \\
2.2 \times 10^{-5} \mathrm{~Hz}^{-1 / 2}\end{array}$ & $\begin{array}{l}8.9 \times 10^{-9} \mathrm{~cm}^{-1} \mathrm{~W} \mathrm{~Hz}^{-1 / 2} \\
2.2 \times 10^{-5} \mathrm{~cm}^{-1} \mathrm{~Hz}^{-1 / 2}\end{array}$ & $3 \mathrm{ppm}$ & $\begin{array}{l}\text { WMS, QEPAS } \\
0.4 \mathrm{~mW}\end{array}$ & [335] \\
\hline & $2.33 \mu \mathrm{m}$ & $\sim 10 \mathrm{~km}$ & $1 / 8 \mathrm{~Hz}$ & $\sim 10^{-3}$ & $10^{-9} \mathrm{~cm}^{-1}$ & $14 \mathrm{ppb}$ & OF-CEAS & [322] \\
\hline & $9.22 \mu \mathrm{m}$ & $11 \mathrm{~cm}$ & $30 \mathrm{~s}$ & $2.0 \times 10^{-7}$ & $\begin{array}{l}2.4 \times 10^{-7} \mathrm{~cm}^{-1} \mathrm{~W} \mathrm{~Hz}^{-1 / 2} \\
1.2 \times 10^{-7} \mathrm{~cm}^{-1} \mathrm{~Hz}^{-1 / 2}\end{array}$ & $0.2 \mathrm{ppb}$ & $\begin{array}{l}\text { WMS, PAS } \\
2 \mathrm{~W}\end{array}$ & [355] \\
\hline & $9.7 \mu \mathrm{m}$ & $1.6 \mathrm{~m}$ & $5 \mathrm{~s}$ & Not stated & Not stated & 80 ppm & NDIR & [129] \\
\hline & $10.34 \mu \mathrm{m}$ & $76 \mathrm{~m}$ & 1s & Not stated & Not stated & $0.2 \mathrm{ppb}$ & Direct TDLS & [388] \\
\hline
\end{tabular}

\begin{tabular}{|l|l|l|l|l|l|l|l|l|}
\hline $\begin{array}{c}\text { benzene } \\
\mathrm{C}_{6} \mathrm{H}_{6}\end{array}$ & $230-260 \mathrm{~nm}$ & $100 \mathrm{~m}$ & $5 \mathrm{~s}$ & Not stated & Not stated & $2 \mathrm{ppm} . \mathrm{m}$ & $\begin{array}{l}\text { Open path } \\
\text { spectrometer }\end{array}$ & {$[163]$} \\
\cline { 2 - 8 } & $239-302 \mathrm{~nm}$ & $740 \mathrm{~m}$ & $\begin{array}{l}120 \mathrm{~s} \text { full } \\
\text { scan }\end{array}$ & $1.4 \times 10^{-2}$ & $1.9 \times 10^{-1} \mathrm{~cm}^{-1}$ & $\begin{array}{l}0.54 \mathrm{ppb} \\
0.4 \mathrm{ppm} . \mathrm{m}\end{array}$ & DOAS & {$[356]$} \\
\cline { 2 - 9 } & $5.1 \mu \mathrm{m}$ & $107 \mathrm{~m}$ & $4 \mathrm{~s}$. & Not stated & Not stated & $1 \mathrm{ppm}$ & $2 \mathrm{WMS}$ & {$[357]$} \\
\hline & $14.8 \mu \mathrm{m}$ & $10 \mathrm{~cm}$ & $1 \mathrm{~s}$ & Not stated & Not stated & $11.5 \mathrm{ppm}$ & Direct TDLS & {$[358]$} \\
\hline
\end{tabular}

\begin{tabular}{|c|c|c|c|c|c|c|c|c|}
\hline \multirow{9}{*}{$\begin{array}{c}\text { carbon } \\
\text { dioxide } \\
\mathrm{CO}_{2}\end{array}$} & $1.532 \mu \mathrm{m}$ & $3 m$ & $6 s$ & $3.5 \times 10^{-5}$ & $1.2 \times 10^{-1} \mathrm{~cm}^{-1}$ & Not stated & $\begin{array}{l}2 f \mathrm{WMS}, \\
\text { waveguides }\end{array}$ & [67] \\
\hline & $1.54 \mu \mathrm{m}$ & $51 \mathrm{~m}$ & $\begin{array}{l}30 \text { min (full } \\
\text { spectrum) }\end{array}$ & $3.1 \times 10^{-3}$ & $1.6 \times 10^{-6} \mathrm{~cm}^{-1}$ & Not stated & BB- CEAS & [359] \\
\hline & $1.5408 \mu \mathrm{m}$ & $\begin{array}{l}2.25 \\
\mathrm{~km}\end{array}$ & $145 \mu \mathrm{s}$ & $\sim 2.5 \times 10^{-4} \mathrm{~Hz}^{-1 / 2}$ & $1.1 \times 10^{-9} \mathrm{~cm}^{-1} \mathrm{~Hz}^{-1 / 2}$ & 500 ppm & CRDS & [360] \\
\hline & $1.570 \mu \mathrm{m}$ & $0.5 \mathrm{~m}$ & $10 \mathrm{~s}$ & Not stated & Not stated & 400 ppm & $\begin{array}{l}\text { Multimode } \\
\text { correlation } 2 f \\
\text { WMS }\end{array}$ & [361] \\
\hline & $1.582 \mu \mathrm{m}$ & $1 \mathrm{~m}$ & $1 \mathrm{~s}$ & $6 \times 10^{-7}$ & $6 \times 10^{-9} \mathrm{~cm}^{-1}$ & 9 ppm & \begin{tabular}{|l|}
$2 f W M S$, \\
balanced \\
receiver
\end{tabular} & [200] \\
\hline & $1.597 \mu \mathrm{m}$ & $\begin{array}{l}\text { Not } \\
\text { stated }\end{array}$ & $2 s$ & $6.8 \times 10^{-4}$ & $5.8 \times 10^{-9} \mathrm{~cm}^{-1} \mathrm{~Hz}^{-1 / 2}$ & Not stated & OF-ICOS & [362] \\
\hline & $1.573 \mu \mathrm{m}$ & $\sim 68 \mathrm{~m}$ & $33 \mathrm{~s}$ & $2.3 \times 10^{-7} \mathrm{~Hz}^{-1 / 2}$ & $3.4 \times 10^{-11} \mathrm{~cm}^{-1} \mathrm{~Hz}^{-1}$ & $3.6 \mathrm{ppm} \mathrm{Hz}^{-1 / 2}$ & $\begin{array}{l}2 f \text { WMS OA- } \\
\text { ICOS }\end{array}$ & [298] \\
\hline & $1.572 \mu \mathrm{m}$ & $8.7 \mathrm{~cm}$ & $2.6 \mathrm{~s}$ & $4.1 \times 10^{-8}$ & $\begin{array}{l}3.6 \times 10^{-9} \mathrm{~cm}^{-1} \\
1.7 \times 10^{-10} \mathrm{~cm}^{-1} \mathrm{~W} \mathrm{~Hz}^{-1 / 2}\end{array}$ & $1.9 \mathrm{ppm}$ & $\begin{array}{l}2 f \mathrm{WMS} \text { PAS } \\
30 \mathrm{~mW}\end{array}$ & [340] \\
\hline & $1.603 \mu \mathrm{m}$ & $\sim 12 \mathrm{~km}$ & $5 \mathrm{~s}$ & $1.9 \times 10^{-5}$ & $1.6 \times 10^{-11} \mathrm{~cm}^{-1} \mathrm{~Hz}^{-1 / 2}$. & $0.1 \mathrm{ppm}$ & CRDS & [363] \\
\hline
\end{tabular}




\begin{tabular}{|c|c|c|c|c|c|c|c|c|}
\hline & \multicolumn{7}{|c|}{ Table 8. Comparison of performance indicators for optical detection of different gases } & \\
\hline$\underline{\text { Gas }}$ & Wave-length & $\frac{\text { Path- }}{\text { length }}$ & \begin{tabular}{|l} 
Response \\
averaging \\
time
\end{tabular} & 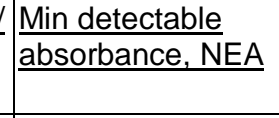 & $\begin{array}{l}\frac{\text { Min detectable }}{\text { absorption coefficient }} \\
\underline{\underline{\alpha}_{\min }}\end{array}$ & \begin{tabular}{|l} 
Min detect- \\
able gas \\
concentration \\
\end{tabular} & Technique & Ref \\
\hline \multirow{7}{*}{$\begin{array}{l}\text { carbon } \\
\text { dioxide } \\
\text { CO2 }\end{array}$} & $1.6055 \mu \mathrm{m}$ & $15.2 \mathrm{~m}$ & $1.9 \mathrm{~s}$ & $3.8 \times 10^{-5} \mathrm{~Hz}^{-1 / 2}$ & $2.5 \times 10^{-8} \mathrm{~cm}^{-1} \mathrm{~Hz}^{-1 / 2}$ & $590 \mathrm{ppm}$ & Direct TDLS & {$[364]$} \\
\hline & $1.997 \mu \mathrm{m}$ & $20 \mathrm{~m}$ & $1.7 \mathrm{~s}$ & Not stated & Not stated & \begin{tabular}{|l|} 
Not stated \\
$1-3 \%$ accuracy
\end{tabular} & Direct TDLS & [365] \\
\hline & $2.0035 \mu \mathrm{m}$ & $5.3 \mathrm{~mm}$ & $1 \mathrm{~s}$ & $\begin{array}{l}1.9 \times 10^{-3} \mathrm{~Hz}^{-1 / 2} \\
7.4 \times 10^{-9} \mathrm{~W} \mathrm{~Hz}^{-1 / 2}\end{array}$ & $\begin{array}{l}3.5 \times 10^{-3} \mathrm{~cm}^{-1} \mathrm{~Hz}^{-1 / 2} \\
1.4 \times 10^{-8} \mathrm{~cm}^{-1} \mathrm{~W} \mathrm{~Hz}^{-1 / 2}\end{array}$ & $18 \mathrm{ppm}$ & $\begin{array}{l}\text { 2fWMS } \\
\text { QEPAS } \\
0.4 \mathrm{~mW}\end{array}$ & [335] \\
\hline & $4.2 \mu \mathrm{m}$ & $24 \mathrm{~mm}$ & $<1 \mathrm{~s}$ & Not stated & Not stated & 1600 ppm & NDIR & [103] \\
\hline & $4.2 \mu \mathrm{m}$ & $1.4 \mathrm{~mm}$ & $10 \mathrm{~s}$ & Not stated & Not stated & 100 ppm & Correlation & [137] \\
\hline & $4.2 \mu \mathrm{m}$ & $40 \mathrm{~mm}$ & $0.1 \mathrm{~s}$ & $<10^{-3}$ & $<2.5 \times 10^{-4} \mathrm{~cm}^{-1}$ & $25 \mathrm{ppm}$ & NDIR & [84] \\
\hline & $4.24 \mu \mathrm{m}$ & $1 \mathrm{~m}$ & $20 \mathrm{~s}$ & Not stated & Not stated & $16 \mathrm{ppb}$ & \begin{tabular}{|l} 
FTIR, \\
waveguide
\end{tabular} & [64] \\
\hline
\end{tabular}

\begin{tabular}{|c|c|c|c|c|c|c|c|c|}
\hline \multirow{12}{*}{$\begin{array}{c}\text { carbon } \\
\text { monoxide } \\
\text { CO }\end{array}$} & $1.582 \mu \mathrm{m}$ & $1 \mathrm{~m}$ & $1 \mathrm{~s}$ & $6.4 \times 10^{-7}$ & $6 \times 10^{-9} \mathrm{~cm}^{-1}$ & $5 \mathrm{ppm}$ & $\begin{array}{l}2 f \mathrm{WMS}, \\
\text { balanced } \\
\text { receiver }\end{array}$ & [200] \\
\hline & $1.564 \mu \mathrm{m}$ & $\begin{array}{l}\text { Not } \\
\text { stated }\end{array}$ & $30 \mathrm{~s}$ & Not stated & $8 \times 10^{-10} \mathrm{~cm}^{-1}$ & $0.9 \mathrm{ppm}$ & \begin{tabular}{|l} 
ICOS, \\
frequency \\
comb
\end{tabular} & [352] \\
\hline & $1.565 \mu \mathrm{m}$ & $4.2 \mathrm{~km}$ & $1 \mathrm{~s}$ & $1.4 \times 10^{-5}$ & $3.1 \times 10^{-11} \mathrm{~cm}^{-1} \mathrm{~Hz}^{-1 / 2}$ & $12 \mathrm{ppb}$ & OA-ICOS & [296] \\
\hline & $1.565 \mu \mathrm{m}$ & $2.7 \mathrm{~km}$ & $1 \mathrm{~s}$ & $5.1 \times 10^{-7} \mathrm{~Hz}^{-1 / 2}$ & $1.9 \times 10^{-12} \mathrm{~cm}^{-1} \mathrm{~Hz}^{-1 / 2}$ & Not stated & OA-ICOS & [292] \\
\hline & $2.3 \mu \mathrm{m}$ & $40 \mathrm{~cm}$ & $4 \mathrm{~s}$ & Not stated & Not stated & $<11 \mathrm{ppm}$ & $2 f \mathrm{WMS}$ & [366] \\
\hline & $2.33 \mu \mathrm{m}$ & $\sim 10 \mathrm{~km}$ & $8 \mathrm{~Hz}$ & $\sim 10^{-3}$ & $10^{-9} \mathrm{~cm}^{-1}$ & $16 \mathrm{ppb}$ & OF-CEAS & [322] \\
\hline & $4.94 \mu \mathrm{m}$ & $1 \mathrm{~m}$ & $40 \mathrm{~s}$ & Not stated & Not stated & \begin{tabular}{|l}
$<400$ ppm \\
$15 \%$ accuracy
\end{tabular} & Direct TDLS & [352] \\
\hline & $4.4-4.8 \mu \mathrm{m}$ & $30 \mathrm{~cm}$ & $3 \mathrm{~s}$ & $1.7 \times 10^{-3}$ & $5.7 \times 10^{-5} \mathrm{~cm}^{-1}$ & $\begin{array}{l}1.1 \mathrm{ppm} . \mathrm{m} \\
2 \% \text { accuracy }\end{array}$ & Direct TDLS & [367] \\
\hline & $4.6 \mu \mathrm{m}$ & $1.4 \mathrm{~mm}$ & $10 \mathrm{~s}$ & Not stated & Not stated & 170 ppm & $\begin{array}{l}\text { Correlation, } \\
\text { FPI }\end{array}$ & {$[137]$} \\
\hline & $4.5524 \mu \mathrm{m}$ & $5.3 \mathrm{~mm}$ & $3 \mathrm{~s}$ & $\begin{array}{l}2.2 \times 10^{-5} \mathrm{~Hz}^{-1 / 2} \\
2.8 \times 10^{-7} \mathrm{~W} \mathrm{~Hz}^{-1 / 2}\end{array}$ & $\begin{array}{l}4.1 \times 10^{-5} \mathrm{~cm}^{-1} \mathrm{~Hz}^{-1 / 2} \\
5.3 \times 10^{-7} \mathrm{~cm}^{-1} \mathrm{~W} \mathrm{~Hz}^{-1 / 2}\end{array}$ & 0.28 ppm & $\begin{array}{l}\text { QEPAS / } 2 f \\
\text { WMS } 13 \mathrm{~mW}\end{array}$ & [368] \\
\hline & $4.633 \mu \mathrm{m}$ & $36 \mathrm{~m}$ & $0.8 \mathrm{~s}$ & $6.3 \times 10^{-5}$ & $5 \times 10^{-9} \mathrm{~cm}^{-1} \mathrm{~Hz}^{-1 / 2}$ & $0.55 \mathrm{ppb}$ & $2 f \mathrm{WMS} Q C L$ & [369] \\
\hline & $4.6024 \mu \mathrm{m}$ & $400 \mathrm{~m}$ & $1 \mathrm{~s}$ & Not stated & Not stated & $\begin{array}{l}0.2 \mathrm{ppb} \\
1 \% \text { accuracy }\end{array}$ & Icos & [302] \\
\hline
\end{tabular}

\begin{tabular}{|l|l|l|l|l|l|l|l|l|}
\hline $\begin{array}{c}\text { ethane } \\
\mathrm{C}_{2} \mathrm{H}_{6}\end{array}$ & $3.348 \mu \mathrm{m}$ & $1.35 \mathrm{~km}$ & $0.5 \mathrm{~s}$ & $1.1 \times 10^{-3} \mathrm{~Hz}^{-1 / 2}$ & $8.1 \times 10^{-9} \mathrm{~cm}^{-1} \mathrm{~Hz}^{-1 / 2}$ & $0.48 \mathrm{ppb} \mathrm{Hz}$ & OA-ICOS & {$[370]$} \\
\cline { 2 - 8 } & $3.34 \mu \mathrm{m}$ & $100 \mathrm{~m}$ & $1 \mathrm{~s}$ & $3.6 \times 10^{-5} \mathrm{~Hz}^{-1 / 2}$ & $3.6 \times 10^{-9} \mathrm{~cm}^{-1} \mathrm{~Hz}^{-1 / 2}$ & $150 \mathrm{ppt}$ & $\begin{array}{l}\text { Herriott } 2 f \\
\text { WMS ICL }\end{array}$ & {$[371]$} \\
\cline { 2 - 9 } & $3.344 \mu \mathrm{m}$. & $201 \mathrm{~m}$ & $1 \mathrm{~s}$ & $2.1 \times 10^{-5}$ & $1.05 \times 10^{-9} \mathrm{~cm}^{-1} \mathrm{~Hz}^{-1 / 2}$ & $70 \mathrm{ppt}$ & $\begin{array}{l}\text { Herriott } 2 f \\
\text { WMS }\end{array}$ & {$[372]$} \\
\cline { 2 - 8 } & $3.3 \mu \mathrm{m}$ & $\begin{array}{l}\sim 3.5 \\
\mathrm{~km}\end{array}$ & $60 \mathrm{~s}$ & $\sim 4.9 \times 10^{-4}$ & $1.4 \times 10^{-9} \mathrm{~cm}^{-1}$ & $70 \mathrm{ppt}$ & CRDS & {$[386]$} \\
\cline { 2 - 8 } & $3.352 \mu \mathrm{m}$ & $\begin{array}{l}\sim 3.5 \\
\mathrm{~km}\end{array}$ & $180 \mathrm{~s}$ & $\sim 6 \times 10^{-5} \mathrm{~Hz}^{-1 / 2}$ & $1.6 \times 10^{-10} \mathrm{~cm}^{-1} \mathrm{~Hz}^{-1 / 2}$ & $6 \mathrm{ppt} \mathrm{Hz}$ & ICOS & {$[236]$} \\
\hline
\end{tabular}

\begin{tabular}{|c|l|l|l|l|l|l|l|l|}
\hline $\begin{array}{c}\text { form- } \\
\text { aldehyde } \\
\mathrm{H}_{2} \mathrm{CO}\end{array}$ & $2.29 \mu \mathrm{m}$ & $26.5 \mathrm{~cm}$ & Not stated & Not stated & Not stated & $<1 \mathrm{ppm}$ & WMS PAS & {$[373]$} \\
\cline { 2 - 9 } & $3.53 \mu \mathrm{m}$ & $83 \mathrm{~m}$ & $3 \mathrm{~s}$ & $2.1 \times 10^{-4} \mathrm{~Hz}^{-1 / 2}[215]$ & $2.1 \times 10^{-8} \mathrm{~cm}^{-1}[215]$ & $50 \mathrm{ppb}$ & OA-ICOS & {$[374]$} \\
\hline & $18 \mathrm{~m}$ & $13 \mathrm{~s}$ & $2 \times 10^{-4}$ & $1 \times 10^{-1} \mathrm{~cm}^{-1}$ & $30 \mathrm{ppb}$ & $\begin{array}{l}\text { Direct TDLS, } \\
\text { DFG }\end{array}$ & {$[375]$} \\
& $3.54 \mu \mathrm{m}$ & $26.5 \mathrm{~cm}$ & Not stated & Not stated & Not stated & $<10 \mathrm{ppb}$ & WMS PAS & {$[373]$} \\
\hline
\end{tabular}




\begin{tabular}{|c|c|c|c|c|c|c|c|c|}
\hline$\underline{\text { Gas }}$ & Wave-length & $\frac{\text { Path- }}{\text { length }}$ & \begin{tabular}{|l} 
Response / \\
averaging \\
time
\end{tabular} & $\begin{array}{l}\text { Min detectable } \\
\text { absorbance, NEA }\end{array}$ & $\begin{array}{l}\frac{\text { Min detectable }}{\text { absorption coefficient, }} \\
\underline{\underline{\alpha_{\min }}}\end{array}$ & \begin{tabular}{|l} 
Min detect- \\
able gas \\
concentration \\
\end{tabular} & Technique & $\underline{\text { Ref }}$ \\
\hline \multirow{5}{*}{$\begin{array}{l}\text { form- } \\
\text { aldehyde } \\
\mathrm{H}_{2} \mathrm{CO}\end{array}$} & $3.56 \mu \mathrm{m}$ & $100 \mathrm{~m}$ & $1 \mathrm{~s}$ & $2.1 \times 10^{-4} \mathrm{~Hz}^{-1 / 2}$ & $2.1 \times 10^{-8} \mathrm{~cm}^{-1} \mathrm{~Hz}^{-1 / 2}$ & $3.5 \mathrm{ppb}$ & $\begin{array}{l}2 f \text { WMS } \\
\text { Herriott }\end{array}$ & [371] \\
\hline & \begin{tabular}{|l|}
$3.52-3.59$ \\
$\mu \mathrm{m}$ \\
\end{tabular} & $4 \mathrm{~cm}$ & $3 \mathrm{~s}$ & $2.5 \times 10^{-8} \mathrm{~W} \mathrm{~Hz}^{-1 / 2}$ & $6.2 \times 10^{-9} \mathrm{Wcm}^{-1} \mathrm{~Hz}^{-1 / 2}$ & $3 \mathrm{ppb}$ & $\begin{array}{l}2 f \text { WMS PAS } \\
65 \mathrm{~mW}\end{array}$ & {$[376]$} \\
\hline & $3.50 \mu \mathrm{m}$ & $1.2 \mathrm{~km}$ & $2 \mathrm{~s}$ & $8 \times 10^{-4}$ & $7 \times 10^{-9} \mathrm{~cm}^{-1}$ & $2 \mathrm{ppb}$ & CW CRDS & [271] \\
\hline & $3.53 \mu \mathrm{m}$ & $5.3 \mathrm{~mm}$ & $10 \mathrm{~s}$ & $\begin{array}{l}1.2 \times 10^{-8} \mathrm{~W} \mathrm{~Hz}^{-1 / 2} \\
3.4 \times 10^{-6} \mathrm{~Hz}^{-1 / 2}\end{array}$ & $\begin{array}{l}2.2 \times 10^{-8} \mathrm{~cm}^{-1} \mathrm{~W} \mathrm{~Hz}^{-1 / 2} \\
6.5 \times 10^{-6} \mathrm{~cm}^{-1} \mathrm{~Hz}^{-1 / 2}\end{array}$ & $0.6 \mathrm{ppb}$ & $\begin{array}{l}\text { QEPAS } \\
3.4 \mathrm{~mW} \text { power }\end{array}$ & [377] \\
\hline & $3.53 \mu \mathrm{m}$ & $100 \mathrm{~m}$ & $260 \mathrm{~s}$ & $1.7 \times 10^{-7}$ & $1.7 \times 10^{-11} \mathrm{~cm}^{-1}$ & $5 \mathrm{ppt}$ & WMS Herriott & [378] \\
\hline \multirow{5}{*}{$\begin{array}{l}\text { Hydrogen } \\
\text { sulfide } \\
\mathrm{H}_{2} \mathrm{~S}\end{array}$} & $190-230 \mathrm{~nm}$ & open & $5 s$ & Not stated & Not stated & 4 ppm.m & $\begin{array}{l}\text { Open path } \\
\text { spectrometer }\end{array}$ & [163] \\
\hline & $1.59 \mu \mathrm{m}$ & $5 \mathrm{~m}$ & Not stated & Not stated & Not stated & $42 \mathrm{ppm}$ & 2fWMS & [379] \\
\hline & $1.578 \mu \mathrm{m}$ & $1 \mathrm{~m}$ & 1s & $5.3 \times 10^{-1}$ & $5.3 \times 10^{-9} \mathrm{~cm}^{-1}$ & $4 \mathrm{ppm}$ & TTFMS & [380] \\
\hline & $1.5745 \mu \mathrm{m}$ & $10 \mathrm{~cm}$ & $3 s$ & $1 \times 10^{-7}$ & $\begin{array}{l}8 \times 10^{-10} \mathrm{~cm}^{-1} \mathrm{~W} \mathrm{~Hz}^{-1 / 2} \\
1 \times 10^{-8} \mathrm{~cm}^{-1}\end{array}$ & $0.17 \mathrm{ppm}$ & $\begin{array}{l}\text { Direct, PAS } \\
40 \mathrm{~mW}\end{array}$ & [345] \\
\hline & $1.5716 \mu \mathrm{m}$ & $1.8 \mathrm{~km}$ & $2 \mathrm{~s}$ & $1 \times 10^{-5}$ & $0.5 \times 10^{-10} \mathrm{~cm}^{-1}$ & $0.22 \mathrm{ppb}$ & OA-ICOS & [381] \\
\hline \multirow{20}{*}{$\begin{array}{l}\text { methane } \\
\mathrm{CH}_{4}\end{array}$} & $1.513 \mu \mathrm{m}$ & $2.3 \mathrm{~km}$ & $0.3 \mathrm{~s}$ & $9 \times 10^{-6} \mathrm{~Hz}^{-1 / 2}$ & $4 \times 10^{-11} \mathrm{~cm}^{-1} \mathrm{~Hz}^{1 / 2}$ & Not stated & NICE-OHMS & [323] \\
\hline & $1.66 \mu \mathrm{m}$ & $\geq 12 \mathrm{~cm}$ & Not stated & $\sim 2 \times 10^{-3}$ & $\sim 2 \times 10^{-4} \mathrm{~cm}^{-1}$ & 100 ppm & NDIR & [382] \\
\hline & $1.684 \mu \mathrm{m}$ & $20 \mathrm{~cm}$ & $2 s$ & Not stated & Not stated & 4.3ppm & Direct TDLS & [184] \\
\hline & $1.6482 \mu \mathrm{m}$ & $74 \mathrm{~m}$ & $1.5-10 \mathrm{~s}$ & $4-8 \times 10^{-5}$ & Not stated & $0.1 \mathrm{ppm}$ & \begin{tabular}{|l|} 
Direct TDLS, \\
Herriott
\end{tabular} & [29] \\
\hline & $1.654 \mu \mathrm{m}$ & $252 \mathrm{~m}$ & $2 s$ & Not stated & Not stated & $20 \mathrm{ppb}$ & $\begin{array}{l}\text { Direct TDLS, } \\
\text { Herriott }\end{array}$ & [383] \\
\hline & $1.659 \mu \mathrm{m}$ & $9.4 \mathrm{~km}$ & $1 \mathrm{~s}$ & Not stated & Not stated & $\pm 1 \mathrm{ppb}$ & OF-CEAS & [321] \\
\hline & $1.651 \mu \mathrm{m}$ & $\sim 12 \mathrm{~km}$ & $5 s$ & $1.9 \times 10^{-5}$ & $1.6 \times 10^{-11} \mathrm{~cm}^{-1} \mathrm{~Hz}^{-1 / 2}$ & $0.5 \mathrm{ppb}$ & CRDS & [363] \\
\hline & $1.654 \mu \mathrm{m}$ & $2.1 \mathrm{~km}$ & $1 \mathrm{~s}$ & $1.4 \times 10^{-5}$ & $6.7 \times 10^{-11} \mathrm{~cm}^{-1} \mathrm{~Hz}^{-1 / 2}$ & $0.3 \mathrm{ppb}$ & OA-ICOS & [296] \\
\hline & $1.73 \mu \mathrm{m}$ & $646 \mathrm{~m}$ & $210 \mathrm{~s}$ & $1.2 \times 10^{-2} \mathrm{~Hz}^{-1 / 2}$ & $1.8 \times 10^{-7} \mathrm{~cm}^{-1} \mathrm{~Hz}^{-1 / 2}$ & $3.4 \mathrm{ppm} \mathrm{Hz}^{-1 / 2}$ & ICOS & [384] \\
\hline & $2.33 \mu \mathrm{m}$ & $\sim 10 \mathrm{~km}$ & $8 \mathrm{~s}$ & $\sim 10^{-3}$ & $10^{-9} \mathrm{~cm}^{-1}$ & $0.4 \mathrm{ppm}$ & OF-CEAS & [322] \\
\hline & $3.3 \mu \mathrm{m}$ & $\sim 4 \mathrm{~cm}$ & $105 \mathrm{~s}$ & Not stated & Not stated & $170 \mathrm{ppm}$ & NDIR & [105] \\
\hline & $3.3 \mu \mathrm{m}$ & $1.4 \mathrm{~mm}$ & $10 \mathrm{~s}$ & Not stated & Not stated & $5 \mathrm{ppm}$ & Correlation & {$[137]$} \\
\hline & $3.3 \mu \mathrm{m}$ & $6.8 \mathrm{~m}$ & $60 \mathrm{~s}$ & $3.5 \times 10^{-4} \mathrm{~Hz}^{-1 / 2}$ & $5.0 \times 10^{-7} \mathrm{~cm}^{-1} \mathrm{~Hz}^{-1 / 2}$ & $15 \mathrm{ppb}$ & \begin{tabular}{|l|} 
Direct, Herriott, \\
balanced \\
detection \\
\end{tabular} & [215] \\
\hline & $3.314 \mu \mathrm{m}$ & $13 \mathrm{~m}$ & $120 \mathrm{~s}$ & $6.5 \times 10^{-4} \mathrm{~Hz}^{-1 / 2}$ & $5.8 \times 10^{-7} \mathrm{~cm}^{-1} \mathrm{~Hz}^{-1 / 2}$ & $3 \mathrm{ppb} \mathrm{Hz}^{-1 / 2}$ & \begin{tabular}{|l|} 
TTFMS, \\
Herriott
\end{tabular} & [385] \\
\hline & $3.2 \mu \mathrm{m}$ & $\begin{array}{l}\sim 3.5 \\
\mathrm{~km}\end{array}$ & $200 \mathrm{~s}$ & $\sim 7 \times 10^{-4}$ & $2.0 \times 10^{-9} \mathrm{~cm}^{-1}$ & $0.16 \mathrm{ppb}$ & CRDS & {$[386]$} \\
\hline & $3.428 \mu \mathrm{m}$ & $13 \mathrm{~m}$ & Not stated & $6.5 \times 10^{-6} \mathrm{~Hz}^{-1 / 2}$ & $5.3 \times 10^{-9} \mathrm{~cm}^{-1} \mathrm{~Hz}^{-1 / 2}$ & $30 \mathrm{ppt} \mathrm{Hz^{-1/2 }}$ & $\begin{array}{l}\text { TTFMS, } \\
\text { Herriott }\end{array}$ & [385] \\
\hline & $7.3 \mu \mathrm{m}$ & $20 \mathrm{~cm}$ & $1.2 \mathrm{MHz}$ & $6 \times 10^{-1} \mathrm{~Hz}^{-1 / 2}$ & $3 \times 10^{-8} \mathrm{~cm}^{-1} \mathrm{~Hz}^{-1 / 2}$ & Not stated & TTFMS & [387] \\
\hline & $7.7 \mu \mathrm{m}$ & $1 \mathrm{~m}$ & $\begin{array}{l}\text { 20s } \\
\text { (instrument }\end{array}$ & Not stated & Not stated & 520 ppb & \begin{tabular}{|l|} 
FTIR, \\
waveguide
\end{tabular} & {$[64]$} \\
\hline & $7.84 \mu \mathrm{m}$ & $1 \mathrm{~km}$ & $1 \mathrm{~s}$ & $5.6 \times 10^{-4} \mathrm{~Hz}^{-1 / 2}$ & $5.6 \times 10^{-9} \mathrm{~cm}^{-1} \mathrm{~Hz}^{-1 / 2}$ & $8 \mathrm{ppb}$ & OF-CEAS & [317] \\
\hline & $8.03 \mu \mathrm{m}$ & $76 \mathrm{~m}$ & 1s & $4.6 \times 10^{-5} \mathrm{~Hz}^{-1 / 2[215]}$ & $6.1 \times 10^{-9} \mathrm{~cm}^{-1} \mathrm{~Hz}^{-1 / 2[215]}$ & $1 \mathrm{ppb}$ & $\begin{array}{l}\text { Direct TDLS, } \\
\text { Herriott }\end{array}$ & [388] \\
\hline
\end{tabular}




\begin{tabular}{|c|c|c|c|c|c|c|c|c|}
\hline$\underline{\text { Gas }}$ & Wave-length & $\frac{\text { Path- }}{\text { length }}$ & \begin{tabular}{|l} 
Response / \\
averaging \\
time
\end{tabular} & $\begin{array}{l}\text { Min detectable } \\
\text { absorbance, NEA }\end{array}$ & 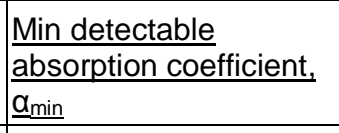 & \begin{tabular}{|l}
$\frac{\text { Min detect- }}{2 \text { able gas }}$ \\
concentration \\
\end{tabular} & Technique & $\underline{\text { Ref }}$ \\
\hline \multirow{13}{*}{$\begin{array}{l}\text { Nitric } \\
\text { oxide } \\
\text { NO }\end{array}$} & $190-230 \mathrm{~nm}$ & $100 \mathrm{~m}$ & $5 s$ & Not stated & Not stated & 4 ppm.m & $\begin{array}{l}\text { Open path } \\
\text { spectrometer }\end{array}$ & [163] \\
\hline & 200-230nm & $\begin{array}{l}\text { Not } \\
\text { stated }\end{array}$ & $3.4 \mathrm{~s}$ & Not stated & Not stated & $5 \mathrm{ppm}$ & DOAS & [389] \\
\hline & $1.81 \mu \mathrm{m}$ & $68 \mathrm{~cm}$ & $10 \mathrm{~s}$ & $4.8 \times 10^{-6}$ & $7 \times 10^{-8} \mathrm{~cm}^{-1}$ & 100 ppm & Direct TDLS & [390] \\
\hline & $5.263 \mu \mathrm{m}$ & $21 \mathrm{~cm}$ & $1.25 \mathrm{~s}$ & $7.2 \times 10^{-6} \mathrm{~Hz}^{-1 / 2}$ & $3.4 \times 10^{-1} \mathrm{~cm}^{-1} \mathrm{~Hz}^{-1 / 2}$ & $2.7 \mathrm{ppm}$ & Direct TDLS & [391] \\
\hline & $5.4 \mu \mathrm{m}$ & $50 \mathrm{~cm}$ & $0.04 \mathrm{~s}$ & $9 \times 10^{-4}$ & $1.8 \times 10^{-5} \mathrm{~cm}^{-1}$ & $\begin{array}{l}0.5 \mathrm{ppm} \cdot \mathrm{m} \\
1 \mathrm{ppm}\end{array}$ & Direct TDLS & [392] \\
\hline & $5.405 \mu \mathrm{m}$ & $9 \mathrm{~m}$ & $0.48 \mathrm{~s}$ & Not stated & Not stated & $13 \mathrm{ppb}$ & $\begin{array}{l}2 f \mathrm{WMS}, \\
\text { waveguide }\end{array}$ & [393] \\
\hline & $5.216 \mu \mathrm{m}$ & $75 m$ & $15 s$ & Not stated & Not stated & $2 \mathrm{ppb}$ & $\begin{array}{l}2 f \text { WMS OA- } \\
\text { ICOS }\end{array}$ & [394] \\
\hline & $5.47 \mu \mathrm{m}$ & $\sim 500 \mathrm{~m}$ & $4 \mathrm{~s}$ & $2.6 \times 10^{-4}$ & $\sim 5.3 \times 10^{-9} \mathrm{~cm}^{-1}$ & $1.2 \mathrm{ppb}$ & ICOS & [395] \\
\hline & $5.263 \mu \mathrm{m}$ & $1.5 \mathrm{~km}$ & $4 s$ & $\sim 6 \times 10^{-4}$ & $\sim 4 \times 10^{-9} \mathrm{~cm}^{-1}$ & $0.7 \mathrm{ppb}$ & ICOS & [396] \\
\hline & $5.26 \mu \mathrm{m}$ & $76 \mathrm{~m}$ & $1 \mathrm{~s}$ & Not stated & Not stated & $0.3 \mathrm{ppb}$ & Direct TDLS & {$[388]$} \\
\hline & $5.405 \mu \mathrm{m}$ & $76 \mathrm{~m}$ & $30 \mathrm{~s}$ & $6.7 \times 10^{-5} \mathrm{~Hz}^{-1 / 2}$ & $8.8 \times 10^{-9} \mathrm{~cm}^{-1} \mathrm{~Hz}^{-1 / 2}$ & $0.2 \mathrm{ppb}$ & $2 f \mathrm{WMS}$ & [397] \\
\hline & $5.263 \mu \mathrm{m}$ & $70 \mathrm{~m}$ & $30 \mathrm{~s}$ & $1.4 \times 10^{-6}$ & $2 \times 10^{-10} \mathrm{~cm}^{-1}$ & $0.1 \mathrm{ppb} \mathrm{Hz}^{-1 / 2}$ & Direct TDLS & [398] \\
\hline & $5.33 \mu \mathrm{m}$ & $\begin{array}{l}\sim 2.4 \\
\mathrm{~km}\end{array}$ & $70 \mathrm{~s}$ & $\sim 5 \times 10^{-5} \mathrm{~Hz}^{-1 / 2}$ & $2 \times 10^{-10} \mathrm{~cm}^{-1} \mathrm{~Hz}^{-1 / 2}$ & $7 \mathrm{ppt}$ & CW CRDS & [399] \\
\hline \multirow[t]{7}{*}{$\begin{array}{l}\text { Nitrous } \\
\text { oxide } \\
\mathrm{N}_{2} \mathrm{O}\end{array}$} & $4.55 \mu \mathrm{m}$ & $5.3 \mathrm{~mm}$ & $3 s$ & \begin{tabular}{|l|}
$4.2 \times 10^{-1} \mathrm{~Hz}^{-1 / 2}$ \\
$8.0 \times 10^{-9} \mathrm{~W} \mathrm{~Hz}^{-1 / 2}$
\end{tabular} & $\begin{array}{l}7.9 \times 10^{-1} \mathrm{~cm}^{-1} \mathrm{~Hz}^{-1 / 2} \\
1.5 \times 10^{-8} \mathrm{~cm}^{-1} \mathrm{~W} \mathrm{~Hz}^{-1 / 2}\end{array}$ & $4 \mathrm{ppb}$ & $\begin{array}{l}\text { QEPAS, } \mathrm{SF}_{6} \\
\text { added } \\
19 \mathrm{~mW} \\
\end{array}$ & {$[368]$} \\
\hline & $4.46 \mu \mathrm{m}$ & $76 \mathrm{~m}$ & $1 \mathrm{~s}$ & $\sim 2 \%$ & Not stated & $0.38 \mathrm{ppb}$ & $\begin{array}{l}\text { Direct TDLS, } \\
\text { Herriott }\end{array}$ & {$[388]$} \\
\hline & $5.4 \mu \mathrm{m}$ & $50 \mathrm{~cm}$ & $0.04 \mathrm{~s}$ & $\sim 5 \times 10^{-5}$ & $\sim 10^{-6} \mathrm{~cm}^{-1}$ & $\begin{array}{l}10 \mathrm{ppm} . \mathrm{m} \\
20 \mathrm{ppm}\end{array}$ & Direct TDLS & [392] \\
\hline & $6.2 \mu \mathrm{m}$ & $4 \mathrm{~m}$ & $10 \mathrm{~s}$ & Not stated & Not stated & $2 \mathrm{ppm}$ & NDIR, White & [117] \\
\hline & $8.0 \mu \mathrm{m}$ & $20 \mathrm{~cm}$ & $1 / 1.2 \mathrm{MHz}$ & $6 \times 10^{-7} \mathrm{~Hz}^{-1 / 2}$ & $3 \times 10^{-8} \mathrm{~cm}^{-1} \mathrm{~Hz}^{-1 / 2}$ & Not stated & TTFMS & [387] \\
\hline & $7.84 \mu \mathrm{m}$ & $1 \mathrm{~km}$ & $1 \mathrm{~s}$ & $5.6 \times 10^{-4} \mathrm{~Hz}^{-1 / 2}$ & $5.6 \times 10^{-9} \mathrm{~cm}^{-1} \mathrm{~Hz}^{-1 / 2}$ & $2 \mathrm{ppb}$ & OF-CEAS & {$[317]$} \\
\hline & $7.87 \mu \mathrm{m}$ & $76 \mathrm{~m}$ & 1s & Not stated & Not stated & $0.16 \mathrm{ppb}$ & $\begin{array}{l}\text { Direct TDLS, } \\
\text { Herriott }\end{array}$ & {$[388]$} \\
\hline \multirow{9}{*}{$\begin{array}{l}\text { Nitrogen } \\
\text { dioxide } \\
\mathrm{NO}_{2}\end{array}$} & 400-450nm & $60 \mathrm{~m}$ & $\begin{array}{l}500 \text { s } \\
\text { (full scan) } \\
\end{array}$ & $2 \times 10^{-4}$ & $3 \times 10^{-8} \mathrm{~cm}^{-1}$ & 14 ppm & DOAS & [400] \\
\hline & $463 \mathrm{~nm}$ & $50 \mathrm{~cm}$ & $2 \mathrm{~s}$ & Not stated & Not stated & 3 ppm & $\begin{array}{l}\mathrm{CCD} \\
\text { spectrometer }\end{array}$ & [401] \\
\hline & $453 \mathrm{~nm}$ & $\begin{array}{l}\lesssim 3.6 \\
\mathrm{~cm}\end{array}$ & $2.1 \mathrm{~s}$ & $\lesssim 5.8 \times 10^{-7} \mathrm{~Hz}^{-1 / 2}$ & $\begin{array}{l}1.6 \times 10^{-7} \mathrm{~cm}^{-1} \mathrm{~Hz}^{-1 / 2} \\
1.4 \times 10^{-6} \mathrm{~cm}^{-1} \mathrm{WHz}^{-1 / 2}\end{array}$ & $10 \mathrm{ppb}$ & $\begin{array}{l}\text { BB PAS } \\
9 W\end{array}$ & {$[337]$} \\
\hline & $411 \mathrm{~nm}$ & $5.4 \mathrm{~km}$ & $0.1 \mathrm{~s}$ & $1.9 \times 10^{-5}$ & $3.5 \times 10^{-y} \mathrm{~cm}^{-1}$ & $200 \mathrm{ppt}$ & OF-CEAS & [402] \\
\hline & $430 \mathrm{~nm}$ & $\begin{array}{l}\text { Not } \\
\text { stated }\end{array}$ & $600 \mathrm{~s}$ & Not stated & $1 \times 10^{-8} \mathrm{~cm}^{-1} \mathrm{~Hz}^{-1 / 2}$ & $300 \mathrm{ppt}$ & BB-PS-CEAS & {$[314]$} \\
\hline & $441-462 \mathrm{~nm}$ & $34.5 \mathrm{~m}$ & $30 \mathrm{~s}$ & $1.3 \times 10^{-5}$ & $3-8 \times 10^{-9} \mathrm{~cm}^{-1}$ & $100-240 \mathrm{ppt}$ & BB-CEAS & [312] \\
\hline & $639 \mathrm{~nm}$ & $50 \mathrm{~cm}$ & $0.1 \mathrm{~s}$ & $6.6 \times 10^{-1}$ & $1.3 \times 10^{-8} \mathrm{~cm}^{-1}$ & \begin{tabular}{|l|}
$5 \mathrm{ppb} . \mathrm{m}$ \\
$10 \mathrm{ppb}$ \\
\end{tabular} & Direct TDLS & [403] \\
\hline & $635 \mathrm{~nm}$ & $160 m$ & $6 s$ & Not stated & Not stated & $7 \mathrm{ppb}$ & $\begin{array}{l}\text { TTFMS open } \\
\text { path }\end{array}$ & [404] \\
\hline & $6.09 \mu \mathrm{m}$ & $2 \mathrm{~km}$ & Not stated & Not stated & Not stated & $1.2 \mathrm{ppb}$ & CRDS & [405] \\
\hline
\end{tabular}




\begin{tabular}{|c|c|c|c|c|c|c|c|c|}
\hline Gas & Wave-length & $\frac{\text { Path- }}{\text { length }}$ & \begin{tabular}{|l} 
Response \\
averaging \\
time
\end{tabular} & $\begin{array}{l}\text { Min detectable } \\
\text { absorbance, NEA }\end{array}$ & $\begin{array}{l}\frac{\text { Min detectable }}{\text { absorption coefficient, }} \\
\underline{\underline{\boldsymbol{\alpha}}_{\min }}\end{array}$ & \begin{tabular}{|l} 
Min detect- \\
able gas \\
concentration \\
\end{tabular} & Technique & Ref \\
\hline \multirow{2}{*}{$\begin{array}{l}\text { Nitrogen } \\
\text { dioxide } \\
\mathrm{NO}_{2}\end{array}$} & $6.25 \mu \mathrm{m}$. & $9.2 \mathrm{~cm}$ & $120 \mathrm{~s}$ & Not stated & Not stated & $0.5 \mathrm{ppb}$ & \begin{tabular}{|l} 
PAS \\
$300 \mathrm{~mW}$ \\
\end{tabular} & [406] \\
\hline & $6.23 \mu \mathrm{m}$ & $40 \mathrm{~m}$ & $1 \mathrm{~s}$ & $6.4 \times 10^{-6}$ & $1.6 \times 10^{-1} \mathrm{~m}^{-1}$ & $75 \mathrm{ppt}$ & 2f WMS, White & [195] \\
\hline \multirow{4}{*}{$\begin{array}{l}\text { Sulfur } \\
\text { dioxide } \\
\mathrm{SO}_{2}\end{array}$} & 190-nm & $100 \mathrm{~m}$ & $5 s$ & Not stated & Not stated & 6 ppm.m & $\begin{array}{l}\text { Open path } \\
\text { spectrometer }\end{array}$ & [163] \\
\hline & $300 \mathrm{~nm}$ & $35 \mathrm{~cm}$ & $3 s$ & Not stated & Not stated & $1 \mathrm{ppm}$ & $\begin{array}{l}\mathrm{CCD} \\
\text { spectrometer }\end{array}$ & [407] \\
\hline & $290-310 \mathrm{~nm}$ & $19 \mathrm{~cm}$ & $60 \mathrm{~s}$ & Not stated & Not stated & 0.4 ppm & Correlation & [135] \\
\hline & $7.505 \mu \mathrm{m}$ & $1.44 \mathrm{~m}$ & $10 \mathrm{~s}$ & $1.8 \times 10^{-4} \mathrm{~Hz}^{-1 / 2}$ & $1.25 \times 10^{-6} \mathrm{~cm}^{-1} \mathrm{~Hz}^{-1 / 2}$ & 2 ppm.m Hz & Direct TDLS & [408] \\
\hline \multirow{8}{*}{$\begin{array}{l}\text { Water } \\
\text { vapour } \\
\mathrm{H}_{2} \mathrm{O}\end{array}$} & $652 \mathrm{~nm}$ & $\begin{array}{l}\sim 10.8 \\
\mathrm{~km}\end{array}$ & $10 \mathrm{~s}$ & $3 \times 10^{-3}$ & $2.9 \times 10^{-9} \mathrm{~cm}^{-1}$ & Not stated & BB-CEAS & [409] \\
\hline & $833.6 \mathrm{~nm}$ & $350 \mathrm{~m}$ & $2 m s$ & $6 \times 10^{-5}$ & $1.7 \times 10^{-9} \mathrm{~cm}^{-1}$ & $1 \mathrm{ppm}$ & CRDS & [410] \\
\hline & $1.396 \mu \mathrm{m}$ & $5.3 \mathrm{~mm}$ & $1 \mathrm{~s}$ & $\begin{array}{l}1.1 \times 10^{-6} \mathrm{~Hz}^{-1 / 2} \\
9.2 \times 10^{-9} \mathrm{~W} \mathrm{~Hz}^{-1 / 2}\end{array}$ & $\begin{array}{l}2.1 \times 10^{-6} \mathrm{~cm}^{-1} \mathrm{~Hz}^{-1 / 2} \\
1.7 \times 10^{-8} \mathrm{~cm}^{-1} \mathrm{~W} \mathrm{~Hz}^{-1 / 2}\end{array}$ & 9 ppm & $\begin{array}{l}\text { QEPAS } \\
8 \mathrm{~mW}\end{array}$ & [411] \\
\hline & $1.393 \mu \mathrm{m}$ & $10 \mathrm{~m}$ & $50 \mathrm{~s}$ & Not stated & Not stated & $1.6 \mathrm{ppb}$ & TTFMS & [412] \\
\hline & $1.3925 \mu \mathrm{m}$. & $46.7 \mathrm{~m}$ & $70 \mathrm{~s}$ & $2 \times 10^{-6}$ & $3.5 \times 10^{-9} \mathrm{~cm}^{-1} \mathrm{~Hz}^{-1 / 2}$ & $70 \mathrm{ppt}$ & \begin{tabular}{|l|}
$2 f$ WMS \\
Herriott
\end{tabular} & [413] \\
\hline & $1.651 \mu \mathrm{m}$ & $\sim 12 \mathrm{~km}$ & $5 s$ & $1.9 \times 10^{-5}$ & $1.6 \times 10^{-11} \mathrm{~cm}^{-1} \mathrm{~Hz}^{-1 / 2}$. & $50 \mathrm{ppm}$ & CRDS & [363] \\
\hline & $1.997 \mu \mathrm{m}$ & $20 \mathrm{~m}$ & $1.7 \mathrm{~s}$ & Not stated & Not stated & Not stated & $\begin{array}{l}\text { Direct TDLS, } \\
\text { Herriott }\end{array}$ & [200] \\
\hline & $6.7 \mu \mathrm{m}$ & $4.2 \mathrm{~km}$ & Up to 100 s & $\begin{array}{l}3.6 \times 10^{-4} \\
\text { (full scan) }\end{array}$ & $2.4 \times 10^{-11} \mathrm{~cm}^{-1} \mathrm{~Hz}^{-1 / 2}$ & $0.28 \mathrm{ppm}$ & OA-ICOS & {$[300]$} \\
\hline
\end{tabular}

\section{References}

[1] Allen MG 1998 Diode laser absorption sensors for gas-dynamic and combustion flows. Meas. Sci. Technol. 9, 545-562.

[2] Laj P et al. 2009 Measuring atmospheric composition change. Atmos. Environ. 43, 5351-5414

[3] Smith D and Španěl P 2007. The challenge of breath analysis for clinical diagnosis and therapeutic monitoring. Analyst 132, 390-396.

[4] Jones E 1987. The pellistor catalytic gas detector. Chapter 2 in Solid state gas sensors, Ed Moseley PT and Tofield BC, Adam Hilger (Bristol).

[5] Williams DE 1987. Conduction and gas response of semiconductor gas sensors. Chapter 4 in Solid state gas sensors, Ed PT Moseley and BC Tofield, Adam Hilger (Bristol).

[6] Bakker E and Telting-Diaz M 2002 Electrochemical sensors. Anal. Chem. 74, 2781-2800.

[7] Lackner M 2007 Tunable diode laser absorption spectroscopy (TDLAS) in the process industries - A review. Rev. Chem. Eng.23, 65-147.

[8] Wild K 2000 Gas quality measurement: A gas control revolution? Gas Eng. Manage. 4012

[9] European standard 2000 Electrical apparatus for the detection and measurement of flammable gases Part 5 Performance requirements for group II apparatus indicating a volume fraction up to $100 \%$ gas. CENELEC: European Committee for Electrotechnical standardization EN 61779-5:2000.

[10] Pyun SH, Cho J, Davidson DF and Hanson RK 2011 Interference-free mid-IR laser absorption detection of methane. Meas. Sci. Technol. 22, 025303 (9pp)

[11] IGEM 1998 Dealing with reported gas escapes. SR/20/Edition 2 (London: The Institution of Gas Engineers and Managers)

[12] Hodgkinson J and Pride RD 2010 Methane-specific gas detectors: the effect of natural gas composition. Meas. Sci. Technol. 21, 105103. 
[13] Hodgkinson J, Shan Q and Pride RD 2006 Detection of a simulated gas leak in a wind tunnel. Meas. Sci. Technol. 17, 1586-1593.

[14] Kannath A, Hodgkinson J, Gillard RG, Riley RJ and Tatam RP 2011 A VCSEL based system for on-site monitoring of low level methane emission. Proc SPIE 7952, 79420F.

[15] Gardiner T, Mead MI, Garcelon S, Robinson R, Swann N, Hansford GM, Woods PT and Jones RL 2010 A lightweight near-infrared spectrometer for the detection of trace atmospheric species. Rev. Sci. Instrum. 81, $083102(11 p p)$

[16] McDermitt D, Burba G, Xu L, Anderson T, Komissarov A, Riensche B, Schedlbauer J, Starr G, Zona D, Oechel W, Oberbauer S and Hastings S 2011 A new low-power, open-path instrument for measuring methane flux by eddy covariance. Appl. Phys. B 102, 391-405.

[17] Banwell CN and McCash EM 1994. Fundamentals of Molecular Spectroscopy. $4^{\text {th }}$ Edition, McGraw-Hill (London).

[18] Ingle JD and Crouch SR 1988. Spectrochemical Analysis. Prentice Hall (London).

[19] Sharpe SW, Johnson TJ, Sams RL, Chu PM, Rhoderick GC and Johnson PA 2004. Gas-phase databases for quantitative infrared spectroscopy. Appl. Spectros. 58, 1452-1461.

[20] Rothman LS et al 2009. The HITRAN 2008 molecular spectroscopic database. J. Quant. Spectrosc. Radiat. 110 (9-10), 533-572.

[21] Rothman LS et al, 1998 The HITRAN molecular spectroscopic database and HAWKS (HITRAN Atmospheric Workstation): 1996 edition. J. Quant. Spectrosc. Radiat. 60, 665-710.

[22] Bomse DS, Stanton AC and Silver JA 1992 Frequency modulation and wavelength modulation spectroscopies: comparison of experimental methods using a lead-salt diode laser. Appl. Opt. 31 (6), 71830.

[23] Kannath A and Rutt HN 2007 Development of low cost instrumentation for non-invasive detection of Helicobacter Pylori. Proc. SPIE 6430 64300L (12pp).

[24] Herriott DR, Kogelnik H and Kompfner R 1964. Off-axis paths in spherical mirror interferometers. Appl. Opt. 3, 523-526.

[25] White JU 1942. Long optical paths of large aperture. J. Opt. Soc. Am. 32, 285-288.

[26] Chernin SM and Barskaya EG 1991. Optical multipass matrix systems. Appl. Opt. 30 (1), 51-58.

[27] Herriott DR and Schulte HJ 1965. Folded optical delay lines. Appl. Opt. 4 (8), 883-889.

[28] McManus JB, Kebabian PL and Zahniser MS 1995. Astigmatic mirror multipass absorption cells for longpath-length spectroscopy. Appl. Opt. 34 (18), 3336-3348.

[29] Gurlit W, Zimmermann R, Giesemann C, Fernholz T, Ebert V, Wolfrum J, Platt U and Burrows JP 2005 Lightweight diode laser spectrometer CHILD (compact high-altitude In-situ laser diode) for balloonborne measurements of water vapor and methane. Appl. Opt. 44 (1), 91-102.

[30] Gazomat 2009. Gazomat Inspectra Laser. Product brochure Rev01 04/09, Gazomat, Bischheim, France.

[31] Werle P and Slemr F 1991. Signal-to-noise ratio analysis in laser absorption spectrometers using optical multipass cells. Appl. Opt. 30, 430-434.

[32] Ofner J, Krüger H-U and Zetzsch C 2010. Circular multireflection cell for optical spectroscopy. Appl. Opt. 49 (26), 5001-5004.

[33] Hodgkinson J, Masiyano D and Tatam RP 2009. Using integrating spheres as absorption cells: pathlength distribution and application of Beer's Law. Appl. Opt. 48 (30), 5748-5758.

[34] Hawe E, Fitzpatrick C, Chambers P, Dooly G and Lewis E 2008. Hazardous gas detection using an integrating sphere as a multipass gas absorption cell. Sensors Actuators A 141, 414-421.

[35] Masiyano D, Hodgkinson J and Tatam RP 2010. Gas cells for tunable diode laser absorption spectroscopy employing optical diffusers. Part 2: Integrating spheres. Appl. Phys. B 100 (2), 303-312.

[36] Toptica 2009, Product specification. Compact Herriott cell for absorption spectroscopy : CMP-30. Toptica Photonics AG, München, Germany, Available at www.toptica.com.

[37] Ferguson DW, Rao KN, Mickelson ME and Larson LE 1993. An experimental study of the 4-0 and 5-0 quadrupole rotation bands of $\mathrm{H}_{2}$ in the visible. J. Mol. Spectrosc. 160, 315-325.

[38] Steyert DW and Sirota JM 2001 Two new long-pass cells for infrared and visible spectroscopy. Rev. Sci. Instrum. 72 (12), 4337-4343.

[39] Robert C 2007. Simple, stable, and compact multiple-reflection optical cell for very long optical paths. Appl. Opt. 46 (22), 5408-5418.

[40] Kasyutich VL 2009. Laser beam patterns of an optical cavity formed by two twisted cylindrical mirrors. Appl Phys B 96, 141-148. 
[41] Tranchart S, Bachir IH and Destombes J-L 1996. Sensitive trace gas detection with near-infrared laser diodes and an integrating sphere. Appl. Opt. 35 (36), 7070-7074.

[42] Wynne RM, Creedon K, Barabadi B, Vedururu S, Merritt J and Ortega A 2008. Simultaneously sensing multiple gases using a single length of hollowcore photonic bandgap fiber with sub-minute response times. Proc. SPIE 7056, 70560W.

[43] Mulrooney J, Clifford J, Fitzpatrick C and Lewis E 2007 Detection of carbon dioxide emissions from a diesel engine using a mid-infrared optical fibre based sensor. Sensors Actuators A 136, 104-110

[44] Hodgkinson J, Pride RD, Tandy C, Moodie DG and Stewart G 2000 Field evaluation of a multipoint fiber optic sensor array for methane detection (OMEGA). Proc SPIE 4074, 90-98.

[45] European Commission 1994 Directive 94/9/EC on equipment and protective systems intended for use in potentially explosive atmospheres (ATEX). Available at http://ec.europa.eu/enterprise/sectors/mechanical/documents/legislation/atex/

[46] Cenelec 2007 Explosive atmospheres - Part 28: Protection of equipment and transmission systems using optical radiation (IEC 60079-28:2006). European Standard EN 60079-28.

[47] Waechter H, Litman J, Cheung AH, Barnes JA and Loock H-P 2010 Chemical sensing using fiber cavity ring-down spectroscopy. Sensors 10, 1716-1742.

[48] Stewart G, Tandy C, Moodie D, Morante MA and Dong F 1998. Design of a fibre optic multi-point sensor for gas detection. Sensors Actuators B 51, 227-232.

[49] Ho HL, Jin W and Demokan MS 2000. Sensitive, multipoint gas detection using TDM and wavelength modulation spectroscopy. Electron. Lett. 36 (14), 1191-1193.

[50] Završnik M and Stewart G 2000. Coherence addressing of quasi-distributed absorption sensors by the FMCW method. J. Lightwave Technol. 18 (I), 57-65.

[51] Ye F, Qian L and Qi B 2009. Multipoint chemical gas sensing using frequency-shifted interferometry. J. Lightwave Technol. 27 (23), 5356-5364.

[52] Stewart G, Mencaglia A, Philp W and Jin W 1998. Interferometric signals in fiber optic methane sensors with wavelength modulation of the DFB laser source. J. Lightwave Technol. 16 (1), 43-53.

[53] Russell P StJ 2006 Photonic-Crystal Fibers. J. Lightwave Technol. 24 (12), 4729-4749

[54] Ritari T, Tuominen J Ludvigsen H, Petersen JC, Sørensen T, Hansen TP and Simonsen HR 2004 Gas sensing using air-guiding photonic bandgap fibers. Opt. Express 12 (17), 4080-4087

[55] Monro TM, Warren-Smith S, Schartner EP, François A, Heng S, Ebendorff-Heidepriem H and Afshar V S 2010 Sensing with suspended-core optical fibers. Opt. Fiber Technol. 16, 343-356

[56] Lehmann H, Kobelke J, Schuster K, Willsch R, Bartelt H, Amezcua-Correa R and Knight JC 2009 Gas sensing with suspended core fibres and hollow core band gap fibres - a comparative study. Proc SPIE 7503, 75035C (4pp)

[57] Brilland L, Charpentier F, Troles J, Bureau B, Boussard-Plédel C, Adam JL, Méchin D and Trégoat D 2009 Microstructured chalcogenide fibers for biological and chemical detection. case study: a $\mathrm{CO}_{2}$ sensor. Proc SPIE 7503750358 (4pp).

[58] Sanghera JS, Brandon Shaw L and Aggarwal ID 2009 Chalcogenide glass-fiber-based mid-IR sources and applications. IEEE J. Sel. Top. Quantum Electron. 15 (1), 114-119.

[59] Rave E, Ephrat P, Goldberg M, Kedmi E and Katzir A 2004 Silver halide photonic crystal fibers for the middle infrared. Appl. Opt. 43 (11), 2236-2241.

[60] Shephard JD, MacPherson WN, Maier RRJ, Jones JDC, Hand DP, Mohebbi M, George AK, Roberts PJ and Knight JC 2005 Single-mode mid-IR guidance in a hollow-core photonic crystal fiber. Opt. Express 13 (18), 7139-7144.

[61] van Brakel A, Grivas C, Petrovich MN and Richardson DJ 2007 Micro-channels machined in microstructured optical fibers by femtosecond laser. Opt. Express 15 (14), 8731-8736.

[62] Désévédavy F, Renversez G, Troles J, Houizot P, Brilland L, Vasilief I, Coulombier Q, Traynor N, Smektala F and Adam J-L 2010 Chalcogenide glass hollow core photonic crystal fibers. Opt. Mater. 32, 1532-1539.

[63] Alfeeli B, Pickrell G and Wang AB 2006 Sub-nanoliter spectroscopic gas sensor. Sensors 6 (10), 13081320.

[64] Kim S-S, Menegazzo N, Young C, Chan J, Carter C and Mizaikoff B 2009 Mid-infrared trace gas analysis with single-pass Fourier transform infrared hollow waveguide gas sensors. Appl. Spectrosc. 63 (3), 331 336.

[65] Eckhardt HS, Dominick H, Frank M, Grattan KTV and Klein K-F 2004 Gas analysis in the UV-region: a hollow core waveguide sensor system. Proc. SPIE 5502, 267-270. 
[66] Charlton C, de Melas F, Inberg A, Croitoru N and Mizaikoff B 2003 Hollow-waveguide gas sensing with room-temperature quantum cascade lasers. IEE Proc.-Optoelectron. 150 (4), 306-309

[67] Fetzer GJ, Pittner AS, Ryder WL and Brown DA 2002 Tunable diode laser absorption spectroscopy in coiled hollow optical waveguides. Appl. Opt. 41 (18), 3613- 3621

[68] Gensty T, von Staden J, Elsäßer W, Höfling S, Reithmaier JP and Forchel A 2005 System performance of a modern hollow-core optical fiber coupled to a quantum cascade laser: transmission efficiency and relative intensity noise. Proc SPIE 5958, 595804 (7pp)

[69] Parry JP, Griffiths BC, Gayraud N, McNaghten ED, Parkes AM, MacPherson WN and Hand DP 2009 Towards practical gas sensing with micro-structured fibres. Meas. Sci. Technol. 20, 075301 (8pp)

[70] Chen J, Hangauer A, Strzoda R and Amann M-C 2010 Resolution limits of laser spectroscopic absorption measurements with hollow glass waveguides. Appl. Opt. 49 (28) 5254-5261

[71] Benabid F, Couny F, Knight JC, Birks TA and Russell P StJ 2005 Compact, stable and efficient all-fibre gas cells using hollow -core photonic crystal fibres. Nature 434 488-491

[72] Henningsen $\mathrm{J}$ and Hald Jan 2008 Dynamics of gas flow in hollow core photonic bandgap fibers. Appl. Opt. 47 (15), 2790-2797

[73] Ma C, Scott B, Pickrell G and Wang A 2010 Porous capillary tubing waveguide for gas sensing. Opt. Lett. 35 (3), 315-317.

[74] Hensley CJ, Broaddus DH, Schaffer CB and Gaeta AL 2007 Photonic band-gap fiber gas cell fabricated using femtosecond micromachining. Opt. Express 15 (11), 6690-6695

[75] Gayraud N, Kornaszewski ŁW, Stone JM, Knight JC, Reid DT, Hand DP and MacPherson WN 2008 Midinfrared gas sensing using a photonic bandgap fiber. Appl. Opt. 47 (9) 1269- 1277.

[76] Saito M and Kato T 2006 Fast infrared spectrometer for flowing gases by the use of a hollow fiber and a PtSi sensor array. Infrared Phys. Techn. 48, 53-58,.

[77] Carvalho JP, Lehmann H, Bartelt H, Magalhães F, Amezcua-Correa R, Santos JL, Van Roosbroeck J, Araújo FM, Ferreira LA and Knight JC 2009 Remote system for detection of low-levels of methane based on photonic crystal fibres and wavelength modulation spectroscopy. J. Sensors 2009398403 (10pp)

[78] Hoo YL, Liu S, Ho HL and Jin W 2010 Fast Response Microstructured Optical Fiber Methane Sensor With Multiple Side-Openings. IEEE Photon. Technol. Lett. 22 (5), 296-298

[79] Gilway Technical Lamp Inc 1999. Engineering Catalogue 168, Gilway, Woburn, MA, USA.

[80] Infratec $\mathrm{GmbH}$. Pyroelectric and multispectral detectors 2005. Product brochure, Infratec $\mathrm{GmbH}$ (Dresden, Germany).

[81] Fonseca L, Cabruja E, Calaza C, Rubio R, Santander J, Figueras E, Gràcia I, Cané C, Moreno M and Marco S 2004. Feasibility of a flip-chip approach to integrate an IR filter and an IR detector in a future gas detection cell. Microsyst.Technol. 10, 382-386.

[82] See for example, Alphasense Ltd 2009. Technical specification, IRC-A1 carbon dioxide infrared sensor. Ref IRCA1/JUN09, Alphasense, Great Notley, UK.

[83] Smith SD, Vass A, Karpushko F. Hardaway H and Crowder JG 2001. The prospects of LEDs, diode detectors and negative luminescence in infrared sensing of gases and spectroscopy. Phil. Trans. Roy. Soc. Lond. A, 359, 621-634.

[84] Aleksandrov SE, Gavrilov GA, Kapralov AA, Matveev BA, Sotnikova G Yu and Remennyi MA 2009. Simulation of characteristics of optical gas sensors based on diode optopairs operating in the midIR spectral range. Tech. Phys. 54 (6), 874-881.

[85] Li-cor Biosciences $2009 \mathrm{LI}-7500 \mathrm{~A}$ open path $\mathrm{CO}_{2} / \mathrm{H}_{2} \mathrm{O}$ gas analyser. Product specification, Li-cor Biosciences, Lincoln, NA, USA.

[86] Smith SD, Crowder JG and Hardaway HR 2002. Recent developments in the applications of mid-infrared lasers, LEDs and other solid state sources to gas detection. Proc SPIE 4651, 157-172.

[87] Maclsaac D, Kanner G and Anderson G 1999. Basic physics of the incandescent lamp (lightbulb). Phys. Teach. 37, 520-525.

[88] Puton J, Jasek K, Siodlowski B, Knap A and Wiśniewski K 2002. Optimisation of a pulsed IR source for NDIR gas analysers. Opto-Electron. Rev. 10 (2), 97-103.

[89] Puton J, Palko T, Knap A, Jasek K and Siodlowski B 2003. Module for measurement of $\mathrm{CO}_{2}$ concentration in exhaled air. Proc SPIE 5124, 278-282.

[90] Schulz O, Müller G, Lloyd M and Ferber A 2005. Impact of environmental parameters on the emission intensity of micromachined infrared sources. Sensors Actuators A 121, 172-180. 
[91] Spannhake J, Schulz O, Helwig A, Müller G and Doll T 2005. Design, development and operational concept of an advanced MEMS IR source for miniaturized gas sensor systems. Proc. IEEE Sensors Conf. 2005, 762-765.

[92] Weber M, Lerch Ph and Renaud Ph 1997. Improved design for fast modulating IR sources. J. Micromech. Microeng. 7, 210-213.

[93] Laine DC, Al-Jorani MN, Carpenter S and Sedgbeer M 1997. Pulsed wideband IR thermal source. IEE Proc. J 144, 315-322.

[94] Gatzmanga H and Baschant D 1996. Directly modulated infrared light source for optical gas analysers. Tech. Mess. 63, 311-316.

[95] Bauer D, Heeger M, Gebhard M and Benecke W 1996. Design and fabrication of a thermal infrared emitter. Sensors Actuators A 55, 57-63.

[96] Cozzani E, Summonte C, Belsito L, Cardinali GC and Roncaglia A 2007. Design study of micromachined thermal emitters for NDIR gas sensing in the 9-12 $\mu \mathrm{m}$ wavelength range. Proc. IEEE Sensors Conf. 2007, 181-184.

[97] Spannhake J, Helwig A, Müller G, Faglia G, Sberveglieri G, Doll T, Wassner T and Eickhoff M 2007. $\mathrm{SnO}_{2}: \mathrm{Sb}-\mathrm{A}$ new material for high-temperature MEMS heater applications: Performance and limitations. Sensors Actuators B 124, 421-428.

[98] Jasek K, Puton J, Siodlowski B and Knap A 2003. Platinum-black coatings for infrared emitters. Proc SPIE 5124, 92-95.

[99] Enoch S, Simon J-J, Escoubas L, Elalmy Z, Lemarquis F, Torchio P and Albrand G 2005. Simple layer-bylayer photonic crystal for the control of thermal emission. Appl. Phys. Lett. 86, 261101 (3pp).

[100] Lin SY, Moreno J and Fleming JG 2003. Three-dimensional photonic-crystal emitter for thermal photovoltaic power generation. Appl. Phys. Lett. 83, 380-382.

[101] Chan DLC, Soljačić M and Joannopoulos JD 2006. Thermal emission and design in 2D-periodic metallic photonics crystal slab. Opt. Express 14 (19), 8785-8796.

[102] Puscasu I, Pralle M, McNeal M, Daly J, Greenwald A and Johnson E 2005. Extraordinary emission from two-dimensional plasmonic-photonic crystals. J. Appl. Phys. 98, 013531 (6pp).

[103] McNeal MP, Moelders N, Pralle MU, Puscasu I, Last L, Ho W, Greenwald AC, Daly JT, Johnson EA and George T 2002. Development of optical MEMS CO 2 sensors. Proc SPIE 4815, 30-35.

[104] Puscasu I, Johnson E, Pralle M, McNeal M, Daly J and Greenwald A 2004 Photonic crystals enable infrared gas sensors. Proc. SPIE 5515, 58-66.

[105] Aleksandrov S, Gavrilov G, Kapralov A, Karandashov S, Matveev B, Sothikova G and Stus N 2002. Portable optoelectronic gas sensors operating in the mid-IR spectral range $(\lambda=3-5 \mu \mathrm{m})$. Proc SPIE 4680, 188-194.

[106] Danilova TN, Zhurtanov BE, Imenkov AN and Yakovlev YP 2005. Light-emitting diodes based on GaSb alloys for the 1.6-4.4 mu m mid-infrared spectral range. Semiconductors 39 (11) 1235-1266.

[107] Krier A, Yin M, Smirnov V, Batty P, Carrington PJ, Solovev V and Sherstnev V 2008 The development of room temperature LEDs and lasers for the mid-infrared spectral range. Phys. Stat. Sol. (A) 205 (1), 129143.

[108] Golovin AS, Astakhova AP, Kizhaev SS, Il'inskaya ND, Serebrennikova O Yu and Yakovlev Yu P 2010. LEDs based on InAs/InAsSb Heterostructures for $\mathrm{CO}_{2}$ spectroscopy $(\lambda=4.3 \mu \mathrm{m})$. Tech. Phys. Lett. 36 (1) 47-49.

[109] Gong XY, Kan H, Makino T, Watanabe K, lida T, Suzuki H, Aoyama M and Yamaguchi T 2000 Light emitting diodes fabricated from liquid phase epitaxial $\ln A s / \ln A s_{x} P_{1-x-y} S b_{y} / \ln A s_{x} P_{1-x-y} S b_{y}$ and $\ln A s / \ln A s_{1-x} S b_{x}$ multi-layers. Cryst. Res. Technol. 35 (5), 549-555.

[110] Stoyanov ND, Zhurtanov BE, Imenkov AN, Astakhova AP, Mikhailova MP and Yakovlev YP 2007. Highefficiency LEDs based on $\mathrm{n}-\mathrm{GaSb} / \mathrm{p}-\mathrm{GaSb} / \mathrm{n}-\mathrm{GalnAsSb} / \mathrm{P}-\mathrm{AIGaAsSb}$ type-Il thyristor heterostructures. Semiconductors 41 (7), 855-859.

[111] Weik F, Steinmeyer G, Tomma JW, Glatthaar R, Vetter U, Nurnus J and Lambrecht A 2006. A roomtemperature continuous-wave operating midinfrared light emitting device. J. Appl. Phys. 99, 114506.

[112] Das NC 2007. Increase in midwave infrared light emitting diode light output due to substrate thinning and texturing. Appl. Phys. Lett. 90, 011111.

[113] Haigh MK, Nash GR, Smith SJ, Buckle L, Emeny MT and Ashley T 2007. Mid-infrared Al $I_{x} n_{1-x} S b$ lightemitting diodes. Appl. Phys. Lett. 90, 231116.

[114] Kizhayev SS, Zotova NV, Molchanov SS and Yakovlev YP 2002. High-power mid-infrared light emitting diodes grown my MOVPE. IEE Proc. J 149 (1) 36-39. 
[115] Krier A and Sherstnev VV 2000. Powerful interface light emitting diodes for methane gas detection J. Phys. D: Appl. Phys. 33, 101-106.

[116] Ivanov-Omskii VI and Matveev BA 2007. Negative luminescence and devices based on this phenomenon. Semiconductors 41 (3), 247-258.

[117] Crowder JG, Hardaway HR and Elliott CT 2002 Mid-infrared gas detection using optically immersed, roomtemperature, semiconductor devices. Meas. Sci. Technol. 13 882-884.

[118] Matveev BA, Zotova NV, Il'inskaya ND, Karandashev SA, Remennyi MA, Stus' NM, Kovchavtsev AP,

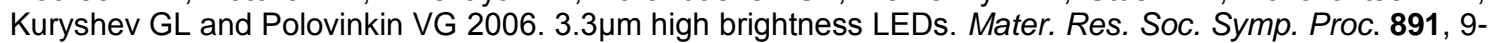
14.

[119] Gevaux D, Green A, Palmer C, Stavrinou P, Roberts C and Phillips C 2003. Resonant-cavity light-emittingdiodes (RC-LEDs) and detectors for mid-IR gas-sensing applications. IEE Proc. J 150 (4) 360-364.

[120] Buss IJ, Nash GR, Rarity JG and Cryan MJ 2010. Finite-difference time-domain modeling of periodic and disordered surface gratings in AllnSb light emitting diodes with metallic back-reflectors. J. Lightwave Technol. 28 (8) 1190-1200.

[121] Nurnus J, Vetter U, Koenig J, Glatthaar R, Lambrecht A, Weik F and Tomm JW 2005. Optically pumped mid infrared emitters built using surface structured PbSe epitaxial layers. Proc SPIE 5840, 212-220.

[122] Sensor Electronic Technology 2008. UVTOP Technical data. Sensor Electronic Technology, Inc. Columbia, SC, USA. Available at http://www.s-et.com.

[123] Hirayama H, Fujikawa S, Noguchi N, Norimatsu J, Takano T, Tsubaki K and Kamata N 2009. 222-282 nm AIGaN and InAIGaN-based deep-UV LEDs fabricated on high-quality AIN on sapphire. Physica Status Solidi (A) 206 (6), 1176-1182.

[124] Taniyasu $Y$ and Kasu M 2008. Aluminum nitride deep-ultraviolet light-emitting $p-n$ junction diodes. Diamond Relat. Mater. 17 1273-1277.

[125] Rumyantsev SL, Sawyer S, Shur MS, Pala N, Bilenko Yu, Zhang JP, Hu X, Lunev A, Deng J and Gaska R 2005. Low-frequency noise of GaN-based ultraviolet light-emitting diodes. J. Appl. Phys. 97, 123107.

[126] Hodgkinson J, Saffell JR and Smith R 2008 Optical absorption gas sensor. European Patent EP1972923 (A2).

[127] Christopher CS and Alexander V 2005. Gas Sensor. Patent WO2005054827 (A1).

[128] Fonollosa J, Rubio R, Hartwig S, Marcoa S, Santander J, Fonseca L, Wöllenstein J and Morenoa M 2008. Design and fabrication of silicon-based mid infrared multi-lenses for gas sensing applications. Sensors Actuators B 132, 498-507.

[129] Fonollosa J, Halford B, Fonseca L, Santander J, Udina S, Moreno M, Hildenbrand J, Wöllenstein J and Marco S 2009. Ethylene optical spectrometer for apple ripening monitoring in controlled atmosphere storehouses. Sensors Actuators B 136, 546-554.

[130] Fonseca L, Rubio R, Santander J, Calaza C, Sabaté N, Ivanov P, Figueras E and Marco S 2009 Qualitative and quantitative substance discrimination using a CMOS compatible non-specific NDIR microarray. Sensors Actuators, B 141 (2), 396-403.

[131] Pyreos 2010 Product information, Pyreos Ltd, Edinburgh, UK. Available at www.pyreos.com.

[132] Gas detection: The professional guide. FLIR Systems Inc, Oregon, USA, 2011. Available from www.flir.com/thermography/

[133] Pride R, Hodgkinson J, Padget M, Van Well B, Strzoda R, Murray S and Ljungberg S-A. Implementation of optical technologies for portable gas leak detection 2004 Proc International Gas Research Conf. (Vancouver), 06-1093-C-pb

[134] Lou XT, Somesfalean G, Chen B, Zhang YG, Wang HS, Zhang ZG, Wu SH and Qin YK 2010. Simultaneous detection of multiple-gas species by correlation spectroscopy using a multimode diode laser. Opt. Lett. 35 (11), 1749-1751.

[135] Lou XT, Somesfalean G, Zhang ZG and Svanberg S 2009 Sulfur dioxide measurements using an ultraviolet light-emitting diode in combination with gas correlation techniques. Appl. Phys. B 94, 699-704.

[136] Dakin JP, Edwards $\mathrm{HO}$ and Weigl BH 1995 Progress with optical gas sensors using correlation spectroscopy. Sensors Actuators B 29, 87-93.

[137] Vargas-Rodríguez $\mathrm{E}$ and Rutt $\mathrm{HN} 2009$ Design of $\mathrm{CO}, \mathrm{CO}_{2}$ and $\mathrm{CH}_{4}$ gas sensors based on correlation spectroscopy using a Fabry-Perot interferometer. Sensors Actuators B 137 410-419

[138] Dakin JP, Gunning MJ, Chambers P and Xin ZJ 2003 Detection of gases by correlation spectroscopy. Sensors Actuators B 90 124-131.

[139] Cheung A, Johnstone W and Moodie D 2006 Gas detection based on optical correlation spectroscopy using a single light source. Meas. Sci. Technol. 17 1107-1112 
[140] Corman T, Kälvesten E, Huiku M, Weckström K, Meriläinen PT and Stemme G 2000 An optical IR-source and $\mathrm{CO}_{2}$-chamber system for $\mathrm{CO}_{2}$ measurements. J. Microelectromech. Syst. 9 (4) 509-516.

[141] Austin E, van Brakel A, Petrovich $M N$ and Richardson DJ 2009 Fibre optical sensor for $\mathrm{C}_{2} \mathrm{H}_{2}$ gas using gas-filled photonic bandgap fibre reference cell. Sensors Actuators B 139 30-34

[142] Kebabian PL, Annen KD, Berkoff TA and Freedman A 2000 Nitrogen dioxide sensing using a novel gas correlation detector. Meas. Sci. Technol. 11, 499-503.

[143] Sandsten J, Edner H and Svanberg S 2004 Gas visualization of industrial hydrocarbon Emissions. Opt. Express 12 (7) 1443-1451.

[144] Levy D and Diken EG 2010. Field identification of unknown gases and vapors via IR spectroscopy for homeland security and defense. IEEE Sensors J. 10 (3), 564-571.

[145] Applied Analytics 2008 Product data for the $\mathrm{H}_{2} \mathrm{~S}-220$ process analyser. Applied Analytics Inc, Concord, MA, USA. Available from http://www.a-a-inc.com/Hydrogen Sulfide.htm.

[146] Stutz J and Platt U 1996. Numerical analysis and estimation of the statistical error of differential optical absorption spectroscopy measurements with least-squares methods. Appl. Opt. 35 (30), 6041-6053.

[147] Xu F, Lv Z, Zhang YG, Somesfalean G and Zhang ZG 2006. Concentration evaluation method using broadband absorption spectroscopy for sulfur dioxide monitoring. Appl. Phys. Lett. 88, 231109.

[148] Degner M, Damaschke N, Ewald H and Lewis E 2010. High resolution LED-spectroscopy for sensor application in harsh environment. Proc IEEE Int. Conf. on Instrumentation \& Measurement Technology, 1382-1386.

[149] Platt U and Stutz J 2008 Differential Optical Absorption Spectroscopy. Principles and Applications. Springer (Berlin)

[150] Hausmann M, Brandenburger U, Brauers T and Dorn H-P 1999. Simple Monte Carlo methods to estimate the spectra evaluation error in differential-optical-absorption spectroscopy. Appl. Opt. 38 (3), 462-475.

[151] Guanghua T, Chuanlong X, Litang S, Bin Z, Daoye Y, Qi P and Shimin W 2009. Improved algorithms of differential optical absorption spectroscopy for monitoring $\mathrm{SO}_{2}, \mathrm{NO}_{2}$ from flue gas. Meas. Sci. Technol. 20, 015601 (8pp).

[152] Wolffenbuttel RF 2004. State-of-the-Art in Integrated Optical Microspectrometers. IEEE Trans. Instrum. Meas. 53 (1), 197-202.

[153] Crocombe RA 2008. Miniature Optical Spectrometers: The Art of the Possible, Part IV: New Near- Infrared Technologies and Spectrometers. Spectroscopy 23 (6), 26-44.

[154] Schuler LP, Milne JS, Dell JM and Faraone L 2009. MEMS-based microspectrometer technologies for NIR and MIR wavelengths. J. Phys. D: Appl. Phys. 42, 133001 (13pp).

[155] Ocean Optics 2007 Product catalogue: Ocean Optics Maya Pro. Ocean Optics Inc (Dunedin, FL, USA).

[156] Crocombe RA, Flanders DC and Atia W 2004. Micro-optical instrumentation for process spectroscopy. Proc. SPIE 5591, 11-25.

[157] Neumann N, Ebermann M, Kurth S and Hiller K 2008. Tunable infrared detector with integrated micromachined Fabry-Perot filter. J. Micro/Nanolith. MEMS MOEMS 7(2), 021004.

[158] Ebermann M, Neumann N, Hiller K, Gittler E, Meinig M and Kurth S 2010. Recent advances in expanding the spectral range of MEMS Fabry-Perot filters. Proc. SPIE 7594, 7540V.

[159] Vaisala 2009. GMT220 Series Carbon Dioxide Transmitters for Industrial Applications. Technical datasheet, Vaisala (Vantaa, Finland). Available from www.vaisala.com.

[160] Chao T-H, Davis SR, Rommel SD, Farca G, Luey B, Martin A and Anderson MH 2009. Compact liquid crystal waveguide based Fourier transform spectrometer for in-situ and remote gas and chemical sensing. Proc. SPIE 7508, 75080K.

[161] Crocombe RA 2008 Miniature Optical Spectrometers: There's Plenty of Room at the Bottom Part I, Background and Mid-Infrared Spectrometers. Spectroscopy 23 (1) 38-56.

[162] See for example, Ocean Optics 2010 USB4000-UV-VIS Miniature Fiber Optic Spectrometer. Product data sheet, Ocean Optics Inc, (Dunedin, FL, USA). Available from www.oceanoptics.com.

[163] Patterson BA, Lenney JP, Sibbett W, Hirst B, Hedges NK and Padgett MJ 1998 Detection of benzene and other gases with an open-path, static Fourier-transform UV spectrometer. Appl. Opt. 37 (15) 3172 - 3175.

[164] Morales JA, Walsh JE, Treacy J and Garland WE 2003 Miniaturized Differential Optical Absorption Spectroscopy (DOAS) system for the analysis of $\mathrm{NO}_{2}$. Proc. SPIE 4876, 1229-1235.

[165] Hirai A, Zeng L and Matsumoto H 2001 Heterodyne Fourier Transform spectroscopy using moving diffraction grating. Japan. J. Appl. Phys. 40, 6138-6142.

[166] Harlander JM, Roesler FL, Cardon JG, Englert CR and Conway RR 2002 SHIMMER: a spatial heterodyne spectrometer for remote sensing of Earth's middle atmosphere. Appl. Opt. 41 (7), 1343-1352. 
[167] Agladze NI and Sievers AJ 2004 Miniaturization of holographic Fourier-transform spectrometers. Appl. Opt. 43 (36), 6568-6579.

[168] Gindele F and Novotny C 2003 Miniaturized fibre-coupled optical spectrometers with temperature compensation based on injection moulding. Proc SPIE 4943, 47-58.

[169] Grabarnik S, Wolffenbuttel R, Emadi A, Loktev M, Sokolova E and Vdovin G 2007. Planar double-grating microspectrometer. Opt. Express 15 (6), 3581-3588.

[170] Emadi A, Grabarnik S, Wu H, de Graaf G, Hedsten K, Enoksson P, Higino Correia J and Wolffenbuttel RF 2010. Spectral measurement using IC-compatible linear variable optical filter. Proc SPIE 7716, $77162 \mathrm{G}$.

[171] DeCorby RG, Ponnampalam N, Epp E, Allen T and McMullin JN 2009. Chip-scale spectrometry based on tapered hollow Bragg waveguides. Opt. Exp. 17 (19), 16632- 16645.

[172] Florjańczyk M, Cheben P, Janz S, Lamontagne B, Lapointe J, Scott A, Solheim B and Xu D-X 2010. Development of slab waveguide spatial heterodyne spectrometer for remote sensing. Proc. SPIE 7594, 75940R-1.

[173] Hult J, Watt RS and Kaminski CF 2007. High bandwidth absorption spectroscopy with a dispersed supercontinuum source. Opt. Express 15 (18), 11385-11395.

[174] Scharf T, Briand D, Bühler S, Manzardo O, Herzig HP and de Rooij NF 2010. Miniaturized Fourier transform spectrometer for gas detection in the MIR region. Sensors Actuators B 147, 116-121.

[175] Reininger FM 2001. The application of large format, broadband quantum well infrared photodetector arrays to spatially modulated prism interferometers. Infrared Phys. Technol. 42, 345-362.

[176] Dell JM, Keating AJ, Milne J, Antoszewski J, Musca CA, Faraone L, Murphy D and Samardzi O 2007. Micro-electromechanical systems-based microspectrometers covering wavelengths from $1500 \mathrm{~nm}$ to 5000nm. Proc. SPIE 6765, 67650L.

[177] Schiff HI, Mackay GI and Bechara J 1994. The use of tunable diode laser absorption spectroscopy for atmospheric measurements, Chapter 5 in Air Monitoring by Spectroscopic Techniques, M W Sigrist, Ed, M Wiley, New York.

[178] Werle P, Slemr F, Maurer K, Kormann R, Mücke R and Jänker B 2002. Near- and mid-infrared laseroptical sensors for gas analysis. Opt. Lasers Eng. 37, 101-114.

[179] Sigrist MW, Bartlome R, Marinov D, Rey JM, Vogler DE and Wächter H 2008. Trace gas monitoring with infrared laser-based detection schemes. Appl. Phys. B 90, 289-300.

[180] SpectraSensors Inc 2008. Product information. SS2100 Hydrogen Sulfide Analyzer. Available from http://www.spectrasensors.com/asp/Site/Products/ByProduct/index.asp,

Also see http://www.neomonitors.com/technology/.

[181] Lackner M, Totschnig G, Winter F, Ortsiefer M, Amann M-C, Shau R and Rosskopf J 2003 Demonstration of methane spectroscopy using a vertical-cavity surface-emitting laser at $1.68 \mu \mathrm{m}$ with up to $5 \mathrm{MHz}$ repetition rate. Meas. Sci. Technol. 14 101-106.

[182] Pride RD, Hodgkinson J, Murray G, Sutton S and Strzoda R 2001. A new portable gas detection instrument based on a novel optical technique. Proc Int. Gas Research conference (Amsterdam), DP04.

[183] Press WH, Teulosky SA, Vetterling WT and Flannery BP 1998. Numerical recipes in $C$ - the art of scientific computing, $2^{\text {nd }}$ Ed, CUP, Cambridge, UK.

[184] Hennig O, Strzoda R, Magori E, Chemisky E, Trump C, Fleischer M, Meixner H and Eisele I 2003. Handheld unit for simultaneous detection of methane and ethane based on NIR-absorption spectroscopy. Sensors Actuators B 95, 151-156.

[185] Swann WC and Gilbert SL 2005 Line centers, pressure shift, and pressure broadening of 1530-1560 nm hydrogen cyanide wavelength calibration lines. J. Opt. Soc. Am. B 22 (8), 1749-1756.

[186] van Well B, Murray S, Hodgkinson J, Pride R, Strzoda R, Gibson G and Padgett M. An open-path, handheld laser system for the detection of methane gas. J. Opt. A: Pure Appl. Opt. 7, S420-S424, 2005.

[187] Chen J, Hangauer A, Strzoda R, Fleischer M and Amann M-C 2009. Miniaturized laser spectroscopic CO sensor for industrial and safety applications. Procedia Chem. Proc. Eurosensors XXIII, 1, 1383-1386.

[188] Chen J, Hangauer A, Strzoda R and Amann M-C 2010 Laser spectroscopic oxygen sensor using diffuse reflector based optical cell and advanced signal processing. Appl. Phys. B 100, 417-425.

[189] Silver JA 1992. Frequency-modulation spectroscopy for trace species detection: theory and comparison among experimental methods, Appl. Opt. 31 (6), 707-717.

[190] McGettrick AJ, Duffin K, Johnstone W, Stewart G and Moodie DG 2008. Tunable diode laser spectroscopy with wavelength modulation: A phasor decomposition method for calibration-free measurements of gas concentration and pressure. J. Lightwave Technol. 26 (1-4), 432-440. 
[191] Chen J, Hangauer A, Strzoda R and Amann M-C 2011 VCSEL-based calibration-free carbon monoxide sensor at $2.3 \mu \mathrm{m}$ with in-line reference cell. Appl Phys B 102 381-389

[192] Silver JA and Stanton AC 1988 Optical interference fringe reduction in laser absorption experiments Appl. Opt. 27 (10), 1914-1916.

[193] Reid J, Shewchun J, Garside BK, Ballik EA 1978 High sensitivity pollution detection employing tunable diode lasers. Appl. Opt. 17 (2), 300-307.

[194] Masiyano D, Hodgkinson J, Schilt S, Tatam RP 2009 Self-mixing interference effects in tunable diode laser absorption spectroscopy. Appl. Phys. B 96 (4), 863-874.

[195] Reid J, El-Sherbiny M, Garside BK, Ballik EA 1980 Sensitivity limits of a tunable diode laser spectrometer, with application to the detection of $\mathrm{NO}_{2}$ at the 100-ppt level. Appl. Opt. 19 (19), 3349-3354.

[196] Carlisle CB, Cooper DE, Prier H 1989. Quantum noise-limited FM spectroscopy with a lead-salt diode laser. Appl. Opt. 28 (13), 2567-2576.

[197] Capellani F, Mellandrone G, Restelli G 1987 In Monitoring of Gaseous Pollutants by Tunable Diode Lasers, ed. Grisar R, Preier H, Schmidtke G, Restelli G (UK: Kluwer Academic Publishers) 51-60.

[198] Chou S, Baer DS, Hanson RK 1997 Diode laser absorption measurements of $\mathrm{CH}_{3} \mathrm{Cl}$ and $\mathrm{CH}_{4}$ near 1.65 um. Appl. Opt. 36 (15), 3288-3293.

[199] Webster CR 1985 Brewster-plate spoiler - a novel method for reducing the amplitude of interferencefringes that limit tunable-laser absorption sensitivities. J. Opt. Soc. Am. B 2 (9), 1464-1470.

[200] Engelbrecht R 2004 A compact NIR fiber-optic diode laser spectrometer for $\mathrm{CO}$ and $\mathrm{CO}_{2}$ : analysis of observed $2 f$ wavelength modulation spectroscopy line shapes. Spectrochim. Acta A 60, 3291-3298.

[201] Lins B Zinn P Engelbrecht R and Schmauss B 2010. Simulation-based comparison of noise effects in wavelength modulation spectroscopy and direct absorption TDLAS. Appl. Phys. B 100, 367-376.

[202] Werle P, Slemr F, Gehrtz M and Braeuchle C 1989 Quantum-limited FM-spectroscopy with a lead-salt diode laser. Appl. Phys. B 49, 99-108.

[203] Andersson M, Persson L, Svensson T and Svanberg S 2007. Flexible lock-in detection system based on synchronized computer plug-in boards applied in sensitive gas spectroscopy. Rev. Sci. Instrum. 78, 113107 (7pp).

[204] So SG, Sani AA, Tittel FK and Wysocki G 2009 Ultra-compact multipass laser absorption spectroscopy platform for distributed sensor networks. Proc Conf. on Lasers and Electro-Optics and Quantum Electronics and Laser Science Conf., 1-5, 702-703.

[205] Chen J. Hangauer A, Strzoda R and Amann M-C 2010 Tunable diode laser spectroscopy with optimum wavelength scanning. Appl Phys B 100, 331-339.

[206] Arita Y and Ewart P 2008 Infra-red multi-mode absorption spectroscopy of acetylene using an Er/Yb:glass micro-laser. Opt. Express 16, 4437-4442.

[207] Ewart P 2008, private communication, Photonex exhibition.

[208] Amann M-C, Arafin S and Vizbaras K 2011 Single mode and tunable GaSb-based VCSELs for wavelengths above $2 \mu \mathrm{m}$. Proc. SPIE 7952, 79520D (7 pp)

[209] Naehle L, Belahsene S,.von Edlinger M, Fischer M, Boissier G, Grech P, Narcy G, Vicet A, Rouillard Y, Koeth $\mathrm{J}$ and Worschech $\mathrm{L} 2011$ Continuous-wave operation of type-I quantum well DFB laser diodes emitting in $3.4 \mathrm{~mm}$ wavelength range around room temperature. Electron. Lett. 47 (1) 46-47

[210] Hosoda T, Kipshidze G, Shterengas L and Belenky G 2010 Diode lasers emitting near $3.44 \mu \mathrm{m}$ in continuous-wave regime at 300K. Electron. Lett. 46 (21) 1455-1456.

[211] Capasso F 2010 High-performance midinfrared quantum cascade lasers. Opt. Eng. 49 (11), 111102

[212] Meyer J and Vurgaftman I 2010 Quantum and Interband Cascade Lasers. Opt. Eng. 49 (11) 111101 (2pp)

[213] Bauer A, Rößner K, Lehnhardt T, Kamp M, Höfling S, Worschech L and Forchel A 2011 Mid-infrared semiconductor heterostructure lasers for gas sensing applications. Semicond. Sci. Technol. 26014032.

[214] Vurgaftman I, Kim M, Kim CS, Bewley WW, Canedy CL, Lindle JR, Abell J and Meyer JR 2010 Challenges for mid-IR interband cascade lasers. Proc. SPIE 7616, 761619 (10pp)

[215] Sonnenfroh DM, Wainner RT, Allen MG and Varner RK 2010 Interband cascade laser-based sensor for ambient $\mathrm{CH}_{4}$. Opt. Eng. 49 (11), 111118 (10pp).

[216] Zorabedian P 1995 Tunable external-cavity semiconductor lasers. Chapter 8 in Tunable Lasers Handbook, Duarte F, Ed, Academic Press (San Diego).

[217] Ricci L, Weidemüller M, Esslinger T, Hemmerich A, Zimmermann C, Vuletic V,. König W and Hänsch TW 1995 A compact grating-stabilized diode laser system for atomic physics Opt. Commun. 117, 541-549.

[218] Littman MG and Metcalf HJ 1978 Spectrally narrow pulsed dye laser without beam expander Appl.Opt. 17 (14), 2224-2227 
[219] Liu AQ and Zhang XM 2007 A review of MEMS external-cavity tunable lasers. J. Micromech. Microeng. 17, R1-R13.

[220] Maute M, Kögel B, Böhm G, Meissner P and Amann M-C 2006 MEMS-tunable 1.55 $\mu m$ VCSEL with extended tuning range incorporating a buried tunnel junction. IEEE Photon. Technol. Lett. 18, 688-690.

[221] Rao GN and Karpf A 2011 External cavity tunable quantum cascade lasers and their applications to trace gas monitoring. Appl. Opt. 50 (4) A100-A115.

[222] See for example, Daylight Solutions, San Diego, CA, USA (www.daylightsolutions.com), Block Engineering, Marlborough, MA, USA (www.blockeng.com) and Pranalytica, Santa Monica, CA, USA (www.pranalytica.com).

[223] Hugi A, Terazzi R, Bonetti Y, Wittmann A, Fischer M, Beck M, Faist J and Gini E 2009 External cavity quantum cascade laser tunable from 7.6 to $11.4 \mu \mathrm{m}$. Appl. Phys. Lett. 95, 061103 (3pp)

[224] Caffey D, Day T, Kim CS, Kim M, Vurgaftman I, Bewley WW, Lindle JR, Canedy CL, Abell J and Meyer JR 2010 Performance characteristics of a continuous wave compact widely tunable external cavity interband cascade lasers. Opt. Express 18 (15) 15691-15696

[225] Svelto O et al [27 authors] 2007 Lasers and Coherent Light Sources, Chapter 11 in Handbook of Lasers and Optics, Träger, F, Ed, Springer (New York).

[226] Vasilyev S, Schiller S, Nevsky A, Grisard A, Fale D, Lallier E, Zhang Z, Boyland AJ, Sahu JK, Ibsen M and Clarkson WA 2008 Broadly tunable single-frequency CW mid-infrared source with milliwatt-level output based on difference-frequency generation in orientation-patterned GaAs. Opt. Lett. 33 (13), 1413-1415.

[227] Breunig I, Haertle D and Buse K 2011 Continuous-wave optical parametric oscillators: recent developments and prospects. Appl Phys B 10, 99-111.

[228] Richter D, Fried A and Weibring P 2009 Difference frequency generation laser based spectrometers. Laser \& Photon. Rev. 3 (4), 343-354.

[229] Richter D and Weibring P 2006 Ultra-high precision mid-IR spectrometer I: Design and analysis of an optical fiber pumped difference-frequency generation source. Appl. Phys. B 82, 479-486.

[230] Asobe M, Tadanaga O, Yanagawa, Umeki T, Nishida Y and Suzuki H 2008 High-power mid-infrared wavelength generation using difference frequency generation in damage-resistant $\mathrm{Zn}: \mathrm{LiNbO}_{3}$ waveguide. Electron. Lett. 44 (4), 288-290.

[231] Fischer C and Sigrist MW 2002 Trace-gas sensing in the 3.3- $\mu \mathrm{m}$ region using a diode-based differencefrequency laser photoacoustic system. Appl. Phys. B 75, 305-310.

[232] Cousin J, Chen W, Bigourd D, Fourmentin M and Kassi S 2009 Telecom-grade fiber laser-based difference-frequency generation and ppb-level detection of benzene vapor in air around $3 \mu \mathrm{m}$. Appl Phys $B$ 97, 919-929.

[233] Barnes NP 1995 Optical Parametric Oscillators. Chapter 7 in Tunable Lasers Handbook, Duarte F, Ed, Academic Press (San Diego).

[234] Müller F, Popp A and Kühnemann F 2003 Transportable, highly sensitive photoacoustic spectrometer based on a continuous-wave dual cavity-optical parametric oscillator. Opt. Express 11 (22), 2820-2825.

[235] Stothard DJM 2010 Practical continuous-wave intracavity optical parametric oscillators, Chapter 16 in Advances in Optical and Photonic Devices, Kim KY, Ed, InTech (Rijeka)

[236] Von Basum G, Halmer D, Hering P, Mürtz M, Schiller S, Müller F, Popp A and Kühnemann F 2004 Parts per trillion sensitivity for ethane in air with an optical parametric oscillator cavity leak-out spectrometer. Opt. Lett. 29 (8), 797-799.

[237] Kaminski CF, Watt RS, Elder AD, Frank JH and Hult J 2008 Supercontinuum radiation for applications in chemical sensing and microscopy. Appl. Phys. B 92, 367-378.

[238] Kurkov AS, Sholokhov EM and Sadovnikova YaE 2011 All-fiber supercontinuum source in the range of 1550 - $2400 \mathrm{~nm}$ based on telecommunication multimode fiber. Laser Phys. Lett. 8 (8), 598-600.

[239] Watt RS, Kaminski CF and Hult J 2008 Generation of supercontinuum radiation in conventional singlemode fibre and its application to broadband absorption spectroscopy. Appl. Phys. B 90, 47-53.

[240] Wadsworth WJ, Ortigosa-Blanch A, Knight JC, Birks TA, Man TPM, and Russell PS 2002 Supercontinuum generation in photonic crystal fibers and optical fiber tapers: a novel light source. J. Opt. Soc. Am. B 19 (9), 2148-2155.

[241] Dudley JM, Genty G and Coen S 2006 Supercontinuum generation in photonic crystal fiber Rev. Mod. Phys. 78 (4), 1135-1184.

[242] Shaw LB, Gattass RR, Sanghera J and Aggarwal I 2011 All-fiber mid-IR supercontinuum source from 1.5 to $5 \mu \mathrm{m}$. Proc. SPIE 7914, 79140P (5pp).

[243] Sanders ST 2002 Wavelength-agile fiber laser using group-velocity dispersion of pulsed super-continua and application to broadband absorption spectroscopy. Appl. Phys. B 75, 799-802. 
[244] Gherman T and Romanini D 2002 Mode-locked cavity-enhanced absorption spectroscopy. Optics Express 10 (19) 1033- 1042.

[245] Adler F, Thorpe MJ, Cossel KC and Ye J 2010 Cavity-enhanced direct frequency comb spectroscopy: technology and applications. Annu. Rev. Anal. Chem. 3, 175-205

[246] Thorpe MJ and Ye J 2008 Cavity-enhanced direct frequency comb spectroscopy. Appl. Phys. B 91, 397414

[247] Adler F, Masłowski P, Foltynowicz A, Cossel KC, Briles TC, Hartl I and Ye J 2010 Mid-infrared Fourier transform spectroscopy with a broadband frequency comb. Opt. Express 18 (21), 21861-21872

[248] Schiller S 2002 Spectrometry with frequency combs. Opt. Lett. 27 (9), 766-768

[249] Coddington I, Swann WC and Newbury NR 2008 Coherent multiheterodyne spectroscopy using stabilized optical frequency combs. Phys. Rev. Lett. 100 (1), 013902.

[250] Coddington I, Swann WC and Newbury NR 2010 Coherent dual-comb spectroscopy at high signal-to-noise ratio. Phys. Rev. A 82 (4), 043817.

[251] Mandon J, Guelachvili G and Picqué N 2009 Fourier transform spectroscopy with a laser frequency comb. Nature Photon. 3 (2), 99-102.

[252] Baumann E, Giorgetta FR, Swann WC, Zolot AM, Coddington I, Newbury NR 2011 Spectroscopy of the methane v3 band with an accurate mid infrared coherent dual-comb spectrometer. Phys. Rev. A 84 (6), 062513.

[253] Schliesser A, Brehm M and Keilmann F 2005 Frequency-comb infrared spectrometer for rapid, remote chemical sensing. Opt. Exp. 13 (22), 9029-9038.

[254] Iseki T, Tai $\mathrm{H}$ and Kimura $\mathrm{K}$ 2000. A portable remote methane sensor using a tunable diode laser. Meas. Sci. Technol. 11, 594-602.

[255] Wainner RT, Green BD, Allen MG, White MA, Stafford-Evans J and Naper R 2002 Handheld, batterypowered near-IR TDL sensor for stand-off detection of gas and vapor plumes. Appl. Phys. B 75, 249-254.

[256] Mitchell D, Duffin K and Johnstone W 2009. Remote methane sensor using tuneable diode laser spectroscopy (TDLS) via a 1w Raman source. Proc SPIE 7503, 750350 (4pp).

[257] Hodgkinson J, van Well B, Padgett M and Pride RD 2006. Modelling and interpretation of gas detection using remote laser pointers. Spectrochim. Acta A 63, 929-939.

[258] Gibson G, van Well B, Hodgkinson J, Pride R, Strzoda R, Murray S, Bishton S and Padgett M 2006 Imaging of methane gas using a scanning, open-path laser system. New J. Phys. 8, 26.

[259] Stothard DJM, Dunn MH and Rae CF 2004 Hyperspectral imaging of gases with a continuous-wave pumpenhanced optical parametric oscillator. Opt. Express 12 (5), 947-955.

[260] Masiyano D, Hodgkinson J and Tatam RP 2010. Gas cells for tunable diode laser absorption spectroscopy employing optical diffusers. Part 1: single and dual pass cells. Appl Phys B 100, 291-302.

[261] Svensson T, Andersson M, Rippe L, Svanberg S, Andersson-Engels S, Johansson J and. Folestad S 2008, VCSEL-based oxygen spectroscopy for structural analysis of pharmaceutical solids Appl. Phys. B 90, 345-354.

[262] Lewander M, Guan Z, Svanberg K, Svanberg S, Svensson T 2009 Clinical system for non-invasive in situ monitoring of gases in the human paranasal sinuses. Opt. Express 17 (13), 10849-10863.

[263] Svensson T, Lewander M and Svanberg S 2010 Laser absorption spectroscopy of water vapor confined in nanoporous alumina: wall collision line broadening and gas diffusion dynamics. Opt. Express 18 (16), 16460-16473.

[264] Masiyano D, Hodgkinson J and Tatam RP 2008 Use of diffuse reflections in tunable diode laser absorption spectroscopy: implications of laser speckle for gas absorption measurements. Appl. Phys. B 90, 279-288.

[265] Paldus BA and Kachanov AA 2005 An historical overview of cavity-enhanced methods. Can. J. Phys. 83, 975-999

[266] Foltynowicz A, Schmidt FM, Ma W and Axner O 2008 Noise-immune cavity-enhanced optical heterodyne molecular spectroscopy: Current status and future potential. Appl. Phys. B 92, 313-316.

[267] O'Keefe A and Deacon DAG 1988 Cavity ring-down optical spectrometer for absorption measurements using pulsed laser sources. Rev. Sci. Instrum. 59 (12), 2544-2551.

[268] Berden G, Peeters R and Meijer G 2000 Cavity ring-down spectroscopy: Experimental schemes and applications. Int. Rev. Phys. Chem. 19 (4), 565-607.

[269] Brown SS 2003 Absorption spectroscopy in high-finesse cavities for atmospheric studies. Chem. Rev. 103, 5219-5238.

[270] Berden G and Engeln R (Eds) 2009 Cavity Ring-Down Spectroscopy: Techniques and Applications. (Chichester, UK: Wiley) 
[271] Dahnke H, von Basum G, Kleinermanns K, Hering $P$ and Mürtz M 2002 Rapid formaldehyde monitoring in ambient air by means of mid-infrared cavity leak-out spectroscopy. Appl. Phys. B 75 (2-3), 311-316.

[272] Bescherer K, Barnes JA, Dias S, Gagliardi G, Loock H-P, Trefiak NR, Waechter H and Yam S 2009 Measurement of multi-exponential optical decay processes by phase-shift cavity ring-down. Appl Phys $B$ 96, 193-200.

[273] Spence TG, Harb CC, Paldus BA, Zare RN, Willke B and Byer RL 2000 A laser-locked cavity ring-down spectrometer employing an analog detection scheme. Rev. Sci. Instrum. 71 (2), 347-353.

[274] Wang C, Srivastava N, Jones BA and Reese RB 2008 A novel multiple species ringdown spectrometer for in situ measurements of methane, carbon dioxide, and carbon isotope. Appl. Phys. B 92, 259-270.

[275] Wang C and Sahay P 2009 Breath Analysis Using Laser Spectroscopic Techniques: Breath Biomarkers, Spectral Fingerprints, and Detection Limits. Sensors 9, 8230-8262.

[276] He Y and Orr BJ 2006 Detection of trace gases by rapidly-swept continuous-wave cavity ringdown spectroscopy: pushing the limits of sensitivity. Appl. Phys. B 85, 355-364.

[277] Huang $\mathrm{H}$ and Lehmann KK 2011 Sensitivity limit of rapidly swept continuous wave cavity ring-down spectroscopy. J. Phys. Chem. A 115, 9411-9421.

[278] Drever RWP, Hall JL, Kowalski FV, Hough J, Ford GM, Munley AJ and Ward H 1983 Laser phase and frequency stabilization using an optical-resonator. Appl. Phys. B 31 (2), 97-105.

[279] Romanini D, Kachanov AA, Morville J and Chenevier M 1999 Measurement of trace gases by diode laser cavity ringdown spectroscopy. Proc SPIE 3821, 94-104.

[280] J Morville, D Romanini, AA Kachanov and M Chenevier 2004 Two schemes for trace detection using cavity ringdown spectroscopy. Appl. Phys. B 78, 465-476.

[281] Engeln R, von Helden G, Berden G and Meijer G 1996 Phase shift cavity ring down absorption spectroscopy. Chemical Physics Letters 262, 105-109.

[282] van Helden JH, Schram DC and Engeln R 2004 Phase-shift cavity ring-down spectroscopy to determine absolute line intensities. Chem. Phys. Lett. 400, 320-325.

[283] Kasyutich V L and Martin PA 2007 On quantitative measurements in phase-shift off-axis cavity-enhanced absorption spectroscopy. Chem. Phys. Lett. 446, 206-211.

[284] Nikolaev IV, Ochkin VN, Spiridonov MV and Tskhai SN 2007 Diode ring-down spectroscopy without intensity modulation in an off-axis multipass cavity. Spectrochim. Acta A 66, 832-835.

[285] Ball SM, Povey IM, Norton EG and Jones RL 2001 Broadband cavity ringdown spectroscopy of the $\mathrm{NO}_{3}$ radical. Chem. Phys. Lett. 342 (1-2), 113-120.

[286] Thompson JE and Myers K 2007 Cavity ring-down lossmeter using a pulsed light emitting diode source and photon counting. Meas. Sci. Technol. 18 147-154

[287] Hamers E, Schram D and Engeln R 2002 Fourier transform phase shift cavity ring down spectroscopy. Chem. Phys. Lett. 365, 237-243.

[288] Petermann C and Fischer P 2011 Actively coupled cavity ringdown spectroscopy with low- power broadband sources. Opt. Express 19 (11), 10164-10173

[289] Halmer D, Von Basum G, Hering $P$ and Mürtz M 2004 Fast exponential fitting algorithm for real-time instrumental use. Rev. Sci. Instrum. 75 (6), 2187-2191.

[290] Mazurenka M, Wada R, Shillings AJL, Butler TJA, Beames JM and AJ Orr-Ewing 2005 Fast Fourier transform analysis in cavity ring-down spectroscopy: application to an optical detector for atmospheric $\mathrm{NO}_{2}$. Appl. Phys. B 81, 135-141.

[291] Boyson TK, Spence TG, Calzada ME and Harb CC 2011 Frequency domain analysis for laser-locked cavity ringdown spectroscopy. Opt. Express 19 (9), 8092- 8101.

[292] Engel GS, Drisdell WS, Keutsch FN, Moyer EJ and Anderson JG 2006 Ultrasensitive near-infrared integrated cavity output spectroscopy technique for detection of $\mathrm{CO}$ at $1.57 \mu \mathrm{m}$ : new sensitivity limits for absorption measurements in passive optical cavities. Appl. Opt. 45 (36), 9221 - 9229.

[293] O'Keefe A 1998 Integrated cavity output analysis of ultra-weak absorption. Chem. Phys. Lett. 293, 331336.

[294] Berden G, Peeters R and Meijer G 1998 Cavity enhanced absorption and cavity enhanced magnetic rotation spectroscopy. Rev. Sci. Instrum. 69 (11), 3763-3769.

[295] O'Keefe A, Scherer JJ and Paul JB 1999 CW integrated cavity output spectroscopy. Chem. Phys. Lett. 307 (5-6), 343-349.

[296] Baer DS, Paul JB, Gupta M and O'Keefe A 2002 Sensitive absorption measurements in the near infrared region using off-axis integrated-cavity-output spectroscopy. Appl. Phys. B 75, 261-265. 
[297] Paul JB, Lapson L and Anderson JG 2001 Ultrasensitive absorption spectroscopy with a high-finesse optical cavity and off-axis alignment. Appl. Opt. 40 (27), 4904-4910

[298] Kasyutich VL, Canosa-Mas CE, Pfrang C, Vaughan S and Wayne 2002 RP Off-axis continuous-wave cavity-enhanced absorption spectroscopy of narrow-band and broadband absorbers using red diode lasers. Appl. Phys. B 75, 755-761.

[299] Zhao W, Gao X, Chen W, Zhang W, Huang T, Wu T and Cha H 2007 Wavelength modulated off-axis integrated cavity output spectroscopy in the near infrared. Appl. Phys. B 86, 353-359.

[300] Moyer EJ, Sayres DS, Engel GS, St Clair JM, Keutsch FN, Allen NT, Kroll JH and Anderson JG 2008 Design considerations in high-sensitivity off-axis integrated cavity output spectroscopy Appl. Phys. B 92, 467-474.

[301] Dyroff C 2011 Otpimum signal-to-noise ratio in off-axis integrated cavity output spectroscopy. Opt. Lett. 36 (7), 1110-1112.

[302] Provencal R, Gupta M, Owano TG, Baer DS, Ricci KN, O'Keefe A and Podolske JR 2005 Cavity-enhanced quantum-cascade laser-based instrument for carbon monoxide measurements. Appl. Opt. 44 (31), 6712 6717.

[303] Gulzow W, Rehder G, Schneider B, von Deimling JS and Sadkowiak B 2011 A new method for continuous measurement of methane and carbon dioxide in surface waters using off-axis integrated cavity output spectroscopy (ICOS): An example from the Baltic Sea. Limnol. Oceanog. Meth. 9, 176-184.

[304] Tuzson B, Hiller RV, Zeyer K, Eugster W, Neftel A, Ammann C and Emmenegger L 2010 Field intercomparison of two optical analyzers for $\mathrm{CH}(4)$ eddy covariance flux measurements. Atmos. Meas. Tech. 3 (6), 1519-1531.

[305] Hancock G and Kasyutich VL 2004 UV cavity enhanced absorption spectroscopy of the hydroxyl radical. Appl. Phys. B 79, 383-388.

[306] Fiedler SE, Hese A and Ruth AA 2003 Incoherent broad-band cavity-enhanced absorption spectroscopy. Chem. Phys. Lett. 371, 284-294.

[307] Orphal $\mathrm{J}$ and Ruth AA 2008 High-resolution Fourier-transform cavity-enhanced absorption spectroscopy in the near-infrared using an incoherent broad-band light source. Opt. Express 16 (23), 19232-19243.

[308] Ball SM, Langridge JM and Jones RL 2004 Broadband cavity enhanced absorption spectroscopy using light emitting diodes. Chem. Phys. Lett. 398, 68-74.

[309] Gherman T, Venables DS, Vaughan S, Orphal J and Ruth AA 2008 Incoherent broadband cavityenhanced absorption spectroscopy in the near-ultraviolet: application to $\mathrm{HONO}$ and $\mathrm{NO}_{2}$. Environ. Sci. Technol. 42, 890-895.

[310] Denzer W, Hamilton ML, Hancock G, Islam M, Langley CE, Peverall R and Ritchie GAD 2009 Nearinfrared broad-band cavity enhanced absorption spectroscopy using a superluminescent light emitting diode. Analyst 134, 2220-2223.

[311] Langridge JM, Laurila T, Watt RS, Jones RL, Kaminski CF and Hult J 2008 Cavity enhanced absorption spectroscopy of multiple trace gas species using a supercontinuum radiation source. Opt. Express 16 (14), 10178-10188.

[312] Langridge JM, Ball SM and Jones RL 2006 A compact broadband cavity enhanced absorption spectrometer for detection of atmospheric $\mathrm{NO}_{2}$ using light emitting diodes. Analyst 131, 916-922.

[313] Laurila T, Burns IS, Hult J, Miller JH and Kaminski CF 2011 A calibration method for broad-bandwidth cavity enhanced absorption spectroscopy performed with supercontinuum radiation Appl Phys B 102, 271278.

[314] Kebabian PL, Herndon SC and Freedman A 2005 Detection of nitrogen dioxide by cavity attenuated phase shift spectroscopy. Anal. Chem. 77, 724-728.

[315] Kebabian PL, Wood EC. Herndon SC and Freedman A 2008 A practical alternative to chemiluminescencebased detection of nitrogen dioxide: cavity attenuated phase shift spectroscopy. Environ. Sci. Technol. 42, 6040-6045

[316] Kasyutich VL, Martin PA and Holdsworth RJ 2006 Phase-shift off-axis cavity-enhanced absorption detector of nitrogen dioxide. Meas. Sci. Technol. 17, 923-931.

[317] Hamilton DJ and Orr-Ewing AJ 2011 A quantum cascade laser-based optical feedback cavity-enhanced absorption spectrometer for the simultaneous measurement of $\mathrm{CH}_{4}$ and $\mathrm{N}_{2} \mathrm{O}$ in air. Appl Phys B 102, 879890.

[318] Morville J, Kassi S, Chenevier M and Romanini D 2005 Fast, low-noise, mode-by-mode, cavity-enhanced absorption spectroscopy by diode-laser self-locking. Appl. Phys. B 80, 1027-1038. 
[319] Motto-Ros V, Durand M and Morville J 2008 Extensive characterization of the optical feedback cavity enhanced absorption spectroscopy (OF-CEAS) technique: ringdown-time calibration of the absorption scale. Appl. Phys. B 91, 203-211.

[320] Hamilton DJ, Nix MGD, Baran SG, Hancock G and Orr-Ewing AJ 2010 Optical feedback cavity-enhanced absorption spectroscopy (OF-CEAS) in a ring cavity. Appl. Phys. B 100 (2), 233-242.

[321] Romanini D, Chenevier M, Kassi S, Schmidt M, Valant C, Ramonet M, Lopez J and Jost H-J 2006 Opticalfeedback cavity-enhanced absorption: a compact spectrometer for real-time measurement of atmospheric methane. Appl. Phys. B 83 (4), 659-667.

[322] Kassi S, Chenevier M, Gianfrani L, Salhi A, Rouillard Y, Ouvrard A and Romanini D 2006 Looking into the volcano with a Mid-IR DFB diode laser and Cavity Enhanced Absorption Spectroscopy. Opt. Express 14 (23), 11442-11452.

[323] Bell CL, Hancock G, Peverall R, Ritchie GAD, van Helden JH and van Leeuwen NJ 2009 Characterization of an external cavity diode laser based ring cavity NICE-OHMS system. Opt. Express 17 (12), 9834-9839.

[324] Foltynowicz A, Ban T, Maslowski P, Adler F and Ye J 2011 Quantum-noise-limited optical frequency comb spectroscopy. Phys. Rev. Lett. 107 (23), 233002

[325] Stewart G, Atherton K, Yu H and Culshaw B 2001. An investigation of an optical fibre amplifier loop for intra-cavity and ring-down cavity loss measurements. Meas. Sci. Technol. 12, 843-849

[326] Stewart G, Shields P and Culshaw B 2004 Development of fibre laser systems for ring-down and intracavity gas spectroscopy in the near-IR. Meas. Sci. Technol. 15, 1621-1628.

[327] von Lerber T and Sigrist MW 2002 Cavity-ring-down principle for fiber-optic resonators: experimental realization of bending loss and evanescent-field sensing. Appl. Opt. 41 (18), 3567-3575.

[328] Ni N, Chan CC, Chuah TK, Xia L and Shum P 2008 Enhancing the measurement accuracy of a cavityenhanced fiber chemical sensor by an adaptive filter. Meas. Sci. Technol. 19 (11), 115203

[329] Elia A, Lugarà PM, Di Franco C and Spagnolo V 2009 Photoacoustic techniques for trace gas sensing based on semiconductor laser sources. Sensors 9, 9616-9628.

[330] Zoltan B, Andrea P and Gabor S 2011 Photoacoustic instruments for practical applications: present, potentials, and future challenges. Appl. Spectrosc. Rev. 46 (1), 1-37.

[331] Hess P 1989 Photoacoustic, photothermal and photochemical processes in gases. (Berlin: Springer).

[332] Hodgkinson J, Johnson M and Dakin JP 2005 Performance of a photothermal detector with turbid liquids. Appl. Opt. 44, 4360-4367.

[333] Schilt, S, Besson, J.-P, Thévenaz, L 2006 Near-infrared laser photoacoustic detection of methane: The impact of molecular relaxation. Appl. Phys. B 82, 319-329.

[334] Kosterev AA, Tittel FK, Serebryakov DV, Malinovsky AL and Morozov IV 2005 Applications of quartz tuning forks in spectroscopic gas sensing. Rev. Sci. Instrum. 76, 043105.

[335] Lewicki R, Wysocki G, Kosterev AA and Tittel FK 2007 Carbon dioxide and ammonia detection using $2 \mu \mathrm{m}$ diode laser based quartz-enhanced photoacoustic spectroscopy. Appl. Phys. B 87, 157-162.

[336] Miklós A, Hess P and Bozóki Z 2001 Application of acoustic resonators in photoacoustic trace gas analysis and metrology. Rev. Sci. Instrum. 72 (4), 1937-1955.

[337] Saarela J, Sorvajärvi T, Laurila T and Toivonen J 2011 Phase-sensitive method for backgroundcompensated photoacoustic detection of $\mathrm{NO}_{2}$ using high-power LEDs. Opt. Express 19 (S4), A725-A732.

[338] Gorelik AV and Starovoitov VS 2009 Small size resonant photoacoustic cell with reduced window background for laser detection of gases. Opt. Spectrosc. 107 (5), 830-835.

[339] Adamson BD, Sader JE and Bieske EJ 2009 Photoacoustic detection of gases using microcantilevers. J. Appl. Phys. 106, 114510 (4pp).

[340] Koskinen V, Fonsen J, Roth K and Kauppinen J 2007 Cantilever enhanced photoacoustic detection of carbon dioxide using a tunable diode laser source. Appl. Phys. B 86, 451-454.

[341] Lindley RE, Parkes AM, Keen KA, Mcnaghten ED and Orr-Ewing AJ 2007 A sensitivity comparison of three photoacoustic cells containing a single microphone, a differential dual microphone or a cantilever pressure sensor. Appl. Phys. B 86, 707-713.

[342] Rey JM, Marinov D, Vogler DE and Sigrist MW 2005 Investigation and optimisation of a multipass resonant photoacoustic cell at high absorption levels. Appl. Phys. B 80, 261-266.

[343] Rossi A, Buffa R, Scotoni M, Bassi D, lannotta S and Boschetti A 2005 Optical enhancement of diode laser-photoacoustic trace gas detection by means of external Fabry-Perot cavity. Appl. Phys. Lett. 87, 041110 (3pp). 
[344] Karioja P, Keränen K, Kautio K, Ollila J, Heikkinen M, Kauppinen I, Kuusela T, Matveev B, McNie ME, Jenkins RM and Palve J 2010 LTCC based differential photo acoustic gas cell for ppm gas sensing. Proc. SPIE $772677260 \mathrm{H}$ (13pp).

[345] Varga A, Bozóki Z, Szakall M and Szabo G 2006 Photoacoustic system for on-line process monitoring of hydrogen sulfide $\left(\mathrm{H}_{2} \mathrm{~S}\right)$ concentration in natural gas streams. Appl. Phys. B 85, 315-321.

[346] Zahniser MS, Nelson DD, McManus JB, Herndon SC, Wood EC, Shorter JH, Lee BH, Santoni GW, Jiménez R, Daube BC, Park S, Kort EA and, Wofsy SC 2009 Infrared QC laser applications to field measurements of atmospheric trace gas sources and sinks in environmental research: enhanced capabilities using continuous wave QCLs. Proc. SPIE 7222, 72220H-1.

[347] Werle P, Mücke R and Slemr F 1993 The limits of signal averaging in atmospheric trace-gas monitoring by tunable diode-laser absorption-spectroscopy (TDLAS). Appl.Phys. B 57, 131-139.

[348] Mount GH, Rumburg B, Havig J, Lamb B, Westberg H, Yonge D, Johnson K and Kincaid R 2002 Measurement of atmospheric ammonia at a dairy using differential optical absorption spectroscopy in the mid-ultraviolet. Atmos. Environ. 36, 1799-1810.

[349] Chambers P, Lyons WB, Lewis E, Sun T and Grattan KTV 2007 The Potential for Development of an NH3 Optical Fibre Gas Sensor. J. Phys. Conf. Ser. 85, 012015.

[350] Claps R, Englich FV, Leleux DP, Richter D, Tittel FK and Curl RF 2001 Ammonia detection by use of nearinfrared diode-laser-based overtone spectroscopy. Appl. Opt. 40 (24) 4387-4394.

[351] Huszár H, Pogány A, Bozóki Z, Mohácsi A, Horváth L and Szabó G 2008 Ammonia monitoring at ppb level using photoacoustic spectroscopy for environmental application. Sensors Actuators B 134 (2), 1027-1033.

[352] Thorpe MJ, Balslev-Clausen D, Kirchner MS and Ye J 2008 Cavity-enhanced optical frequency comb spectroscopy: Application to human breath analysis. Opt. Exp. 16 (4), 2387-2397.

[353] Besson J.-P, Schilt S, Rochat E and Thévenaz L 2006 Ammonia trace measurements at ppb level based on near-IR photoacoustic spectroscopy. Appl. Phys. B 85 (2-3), 323-328.

[354] Paldus BA, Fidric BG, Sanders SS, Tan SM, Pham H, Kachanov AA, Wahl EH and Crosson ER 2004 High sensitivity detectors based on cavity ring-down spectroscopy. Proc. SPIE 5617, 312-322.

[355] Pushkarsky MB, Webber ME, Baghdassarian O, Narasimhan LR and Patel CKN 2002 Laser-based photoacoustic ammonia sensors for industrial applications. Appl. Phys. B 75 (2-3), 391-396.

[356] Lee C, Choi YJ, Jung JS, Lee JS, Kim KH and Kim YJ 2005 Measurement of atmospheric monoaromatic hydrocarbons using differential optical absorption spectroscopy: Comparison with on-line gas chromatography measurements in urban air. Atmos. Environ. 39, 2225-2234.

[357] Jeffers JD, Roller CB, Namjou K, Evans MA, McSpadden L, Grego J and McCann PJ 2004 Real-time diode laser measurements of vapor-phase benzene. Anal. Chem. 76 (2), 424-432.

[358] Chen W, Cazier F, Tittel F and Boucher D 2000 Measurements of benzene concentration by differencefrequency laser absorption spectroscopy. Appl. Opt. 39 (33), 6238-6242.

[359] Watt RS, Laurila T, Kaminski CF and Hult J 2009 Cavity enhanced spectroscopy of high-temperature $\mathrm{H}_{2} \mathrm{O}$ in the near-infrared using a supercontinuum light source. Appl. Spectrosc. 63 (12), 1389-1395.

[360] He Y and Orr BJ 2004 Rapid measurement of cavity ringdown absorption spectra with a swept-frequency laser. Appl. Phys. B 79, 941-945.

[361] Lou X, Somesfalean G and Zhang Z 2008 Gas detection by correlation spectroscopy employing a multimode diode laser. Appl. Opt. 47 (13), 2392-2398.

[362] Baran SG, Hancock G, Peverall R, Ritchie GAD and van Leeuwen NJ 2009 Optical feedback cavity enhanced absorption spectroscopy with diode lasers. Analyst 134, 243 - 249.

[363] Crosson ER 2008 A cavity ring-down analyzer for measuring atmospheric levels of methane, carbon dioxide, and water vapour. Appl. Phys. B 92, 403-408.

[364] Kasyutich VL and Martin PA 2007 Multipass optical cell based upon two cylindrical mirrors for tunable diode laser absorption spectroscopy. Appl. Phys. B 88, 125-130.

[365] Rocco A, de Natale G, Gagliardi G and Gianfrani L 2004 A diode-laser based spectrometer for in-situ measurements of volcanic gases. Appl. Phys. B 78, 235-240.

[366] Hangauer A, Chen J, Strzoda R, Ortsiefer M and Amann M.-C 2008 Wavelength modulation spectroscopy with a widely tunable InP-based $2.3 \mu \mathrm{m}$ vertical-cavity surface-emitting laser. Opt. Lett. 33 (14), 15661568.

[367] Barron-Jimenez R, Caton JA, Anderson TN, Lucht RP, Walther T, Roy S, Brown MS and Gord JR 2006 Application of a difference-frequency-mixing based diode-laser sensor for carbon monoxide detection in the 4.4-4.8 um spectral region. Appl. Phys. B 85 (2-3), 185-197. 
[368] Kosterev AA, Bakhirkin YA and Tittel FK 2005 Ultrasensitive gas detection by quartz-enhanced photoacoustic spectroscopy in the fundamental molecular absorption bands region. Appl. Phys. B 80 (1) 133-138.

[369] Kormann R, Königstedt R, Parchatka U, Lelieveld J and Fischer H 2005 QUALITAS: A mid-infrared spectrometer for sensitive trace gas measurements based on quantum cascade lasers in $\mathrm{CW}$ operation. Rev. Sci. Instrum. 76 (7) 1-8.

[370] Parameswaran KR, Rosen, DI, Allen MG, Ganz AM and Risby TH 2009 Off-axis integrated cavity output spectroscopy with a mid-infrared interband cascade laser for real-time breath ethane measurements. Appl. Opt. 48 (4), B73-B79.

[371] Wysocki G, Bakhirkin Y, So S, Tittel FK, Hill CJ, Yang RQ and Fraser MP 2007 Dual interband cascade laser based trace-gas sensor for environmental monitoring. Appl. Opt. 46 (33), 8202-8210.

[372] Patterson CS, McMillan LC, Longbottom C, Gibson GM, Padgett MJ and Skeldon KD 2007 Portable optical spectroscopy for accurate analysis of ethane in exhaled breath. Meas. Sci. Technol. 18 1459-1464

[373] Cihelka J, Matulková I and Civiš S 2009 Laser diode photoacoustic and FTIR laser spectroscopy of formaldehyde in the $2.3 \mu \mathrm{m}$ and $3.5 \mu \mathrm{m}$ spectral range. J. Mol. Spectrosc. 256 (1), 68-74.

[374] Miller JH, Bakhirkin YA, Ajtai T, Tittel FK, Hill CJ and Yang RQ 2006 Detection of formaldehyde using offaxis integrated cavity output spectroscopy with an interband cascade laser. Appl. Phys. B 85 (2-3), 391396.

[375] Lancaster DG, Richter D, Curl RF and Tittel FK 1998 Real-time measurements of trace gases using a compact difference-frequency-based sensor operating at 3:5 $\mu \mathrm{m}$. Appl. Phys. B 67, 339-345.

[376] M Angelmahr, A Miklós and P Hess 2006 Photoacoustic spectroscopy of formaldehyde with tunable laser radiation at the parts per billion level. Appl. Phys. B 85, 285-288.

[377] Horstjann M, Bakhirkin YA, Kosterev AA, Curl RF, Tittel FK, Wong CM, Hill CJ and Yang RQ 2004 Formaldehyde sensor using interband cascade laser based quartz-enhanced photoacoustic spectroscopy. Appl. Phys. B 79, 799-803.

[378] Weibring P, Richter D, Fried A, Walega JG, Dyroff C 2006 Ultra-high-precision mid-IR spectrometer II: System description and spectroscopic performance. Appl. Phys. B 85 (2-3), 207-218.,

[379] Weldon V, O'Gorman J, Phelan P, Hegarty J and Tanburn-Ek T $1995 \mathrm{H}_{2} \mathrm{~S}$ and $\mathrm{CO}_{2}$ gas sensing using DFB laser diodes emitting at 1.57um. Sensors Actuators B 29, 101-107.

[380] G Modugno, C Corsi, M Gabrysch and $M$ Inguscio. Detection of $\mathrm{H}_{2} \mathrm{~S}$ at the ppm level using a telecommunication diode laser. Opt. Commun. 4755, 76-80, 1998.

[381] Chen W, Kosterev AA, Tittel FK, Gao X, Zhao W $2008 \mathrm{H}_{2} \mathrm{~S}$ trace concentration measurements using offaxis integrated cavity output spectroscopy in the near-infrared. Appl. Phys. B 90 (2), 311-315.

[382] Massie C, Stewart G, McGregor G and Gilchrist JR 2006 Design of a portable optical sensor for methane gas detection Sensor. Actuat. B 113 830-836.

[383] Richard EC, Kelly KK, Winkler RH, Wilson R, Thompson TL, McLaughlin RJ, Schmeltekopf AL and Tuck AF 2002 A fast-response near-infrared tunable diode laser absorption spectrometer for in situ measurements $\mathrm{CH}_{4}$ in the upper troposphere and lower stratosphere. Appl. Phys. B 75 (2-3) 183-194.

[384] Barry HR, Corner L, Hancock G, Peverall R and Ritchie GAD 2001 Cavity-enhanced absorption

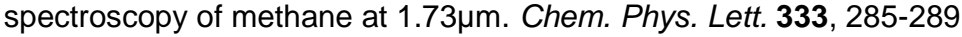

[385] Maddaloni P, Malara P, Gagliardi G, de Natale P 2006 Two-tone frequency modulation spectroscopy for ambient-air trace gas detection using a portable difference-frequency source around $3 \mu \mathrm{m}$. Appl. Phys. $B$ 85 (2-3), 219-222.

[386] Ngai AKY, Persijn ST, von Basum G, Harren FJM 2006 Automatically tunable continuous-wave optical parametric oscillator for high-resolution spectroscopy and sensitive trace-gas detection Appl. Phys. B 85 (2-3), 173-180.

[387] Borri S, Bartalini S, de Natale P, Inguscio M, Gmachl C, Capasso F, Sivco DL and Cho AY 2006 Frequency modulation spectroscopy by means of quantum-cascade lasers. Appl. Phys. B 85, 223-229.

[388] Mcmanus JB, Shorter H, Nelson DD, Zahniser MS, Glenn DE and McGovern RM 2008 Pulsed quantum cascade laser instrument with compact design for rapid, high sensitivity measurements of trace gases in air. Appl. Phys. B 92, 387-392.

[389] Dooly G, Fitzpatrick C and Lewis E 2008 Deep UV based DOAS system for the monitoring of nitric oxide using ratiometric separation techniques. Sensors Actuators, $B 134$ (1), 317-323.

[390] Sonnenfroh DM and Allen MG 1997 Absorption measurements of the second overtone band of NO in ambient and combustion gases with a 1.8-mm room-temperature diode laser. Appl. Opt. 36 (30), 79707977 
[391] Kasyutich VL, Holdsworth RJ and Martin PA 2008 Mid-infrared laser absorption spectrometers based upon all-diode laser difference frequency generation and a room temperature quantum cascade laser for the detection of $\mathrm{CO}, \mathrm{N}_{2} \mathrm{O}$ and NO. Appl. Phys. B 92 (2), 271-279.

[392] Sonnenfroh DM, Rawlins WT, Allen MG, Gmachl C, Capasso F, Hutchinson AL, Sivco DL, Baillargeon JN and Cho AY 2001 Application of balanced detection to absorption measurements of trace gases with room-temperature, quasi-CW quantum-cascade lasers. Appl. Opt. 40 (6), 812-820.

[393] Fetzer GJ, Pittner AS and Silkoff PE 2003 Mid-infrared laser absorption spectroscopy in coiled hollow optical waveguides. Proc.SPIE 4957, 124-133.

[394] Bakhirkin YA, Kosterev AA, Roller C, Curl RF and Tittel FK 2004 Mid-infrared quantum cascade laser based off-axis integrated cavity output spectroscopy for biogenic nitric oxide detection. Appl. Opt. 43, 2257-2266.

[395] McCurdy MR, Bakhirkin YA and Tittel FK 2006 Quantum cascade laser-based integrated cavity output spectroscopy of exhaled nitric oxide. Appl. Phys. B 85 (2-3), 445-452.

[396] Silva ML, Sonnenfroh DM, Rosen DI, Allen MG and O'Keefe A 2005 Integrated cavity output spectroscopy measurements of nitric oxide levels in breath with a pulsed room-temperature quantum cascade laser. Appl. Phys. B 81 (5), 705-710.

[397] Moeskops BWM, Cristescu SM and Harren FJM 2006 Sub-part-per-billion monitoring of nitric oxide by use of wavelength modulation spectroscopy in combination with a thermoelectrically cooled, continuous-wave quantum cascade laser. Opt. Lett. 31 (6), 823-825.

[398] Mcmanus JB, Nelson DD, Herndon SC, Shorter JH, Zahniser MS, Blaser S, Hvozdara L, Muller A, Giovannini M and Faist $\mathrm{J} 2006$ Comparison of $\mathrm{cW}$ and pulsed operation with a TE-cooled quantum cascade infrared laser for detection of nitric oxide at $1900 \mathrm{~cm}^{-1}$. Appl. Phys. B 85, 235-241.

[399] Heinrich K, Fritsch T, Hering $P$ and Mürtz M 2009 Infrared laser-spectroscopic analysis of ${ }^{14} \mathrm{NO}$ and ${ }^{15} \mathrm{NO}$ in human breath. Appl. Phys. B 95 (2), 281-286.

[400] Cheng AYS and Chan MH 2004 Acousto-optic differential optical absorption spectroscopy for atmospheric measurement of nitrogen dioxide in Hong Kong. Appl. Spectrosc. 58, 1462-1468.

[401] Xu F, Lv Z, Lou X, Zhang Y and Zhang Z 2008 Nitrogen dioxide monitoring using a blue LED. Appl. Opt. 47 (29), 5337-5340.

[402] Courtillot I, Morville J, Motto-Ros V and Romanini D 2006 Sub-ppb NO 2 detection by optical feedback cavity-enhanced absorption spectroscopy with a blue diode laser. Appl. Phys. B 85, 407-412

[403] Sonnenfroh DM and Allen MG 1996 Ultrasensitive, visible tunable diode laser detection of $\mathrm{NO}_{2}$. Appl. Opt. 35, 4053-4058.

[404] Somesfalean G, Alnis J, Gustafsson U, Edner H and Svanberg S 2005 Long-path monitoring of $\mathrm{NO}_{2}$ with a $635 \mathrm{~nm}$ diode laser using frequency-modulation spectroscopy. Appl. Opt. 44, 5148-5151.

[405] Rao GN and Karpf A 2010 High sensitivity detection of $\mathrm{NO}_{2}$ employing cavity ringdown spectroscopy and an external cavity continuously tunable quantum cascade laser. Appl. Opt. 49 (26), 4906-4914.

[406] Pushkarsky M, Tsekoun A, Dunayevskiy IG, Go R and Patel CKN 2006 Sub-parts-per-billion level detection of $\mathrm{NO}_{2}$ using room-temperature quantum cascade lasers. Proc Nat. Acad. Sci. USA 103 (29), 10846-10849.

[407] Xu F, Lv Z, Zhang YG, Somesfalean G and Zhang ZG 2006 Concentration evaluation method using broadband absorption spectroscopy for sulfur dioxide monitoring. Appl. Phys. Lett. 88, 231109.

[408] Rawlins WT, Hensley JM, Sonnenfroh DM, Oakes DB and Allen MG 2005 Quantum cascade laser sensor for $\mathrm{SO}_{2}$ and $\mathrm{SO}_{3}$ for application to combustor exhaust streams. Appl. Opt. 44, 6635-6643.

[409] Langridge JM, Ball SM., Shillings AJL and Jones RL 2008 A broadband absorption spectrometer using light emitting diodes for ultrasensitive, in situ trace gas detection. Rev. Sci. Instrum. 79 (12) 123110

[410] Levenson MD, Paldus BA, Spence TG, Harb CC, Harris Jr JS and Zare RN 1998 Optical heterodyne detection in cavity ring-down spectroscopy. Chem. Phys. Lett. 290 335-340.

[411] Liu K, Li J, Wang L, Tan T, Zhang W, Gao X, Chen W and Tittel FK 2009 Trace gas sensor based on quartz tuning fork enhanced laser photoacoustic spectroscopy. Appl. Phys. B 94 (3), 527-533.

[412] Edwards CS, Barwood GP, Bell SA, Gill P and Stevens M 2001 A tunable diode laser absorption spectrometer for moisture measurements in the low parts in $10^{9}$ range. Meas. Sci. Technol. 12, 12141218.

[413] Hovde DC, Hodges JT, Scace GE and Silver JA 2001 Wavelength-modulation laser hygrometer for ultrasensitive detection of water vapor in semiconductor gases Appl. Opt. 40 (6) 829-839. 